\title{
Wavelet Regularization and the Continuous Relaxation Spectrum.
}

\author{
N. J. Goulding.
}


UMI Number: U574416

All rights reserved

\section{INFORMATION TO ALL USERS}

The quality of this reproduction is dependent upon the quality of the copy submitted.

In the unlikely event that the author did not send a complete manuscript and there are missing pages, these will be noted. Also, if material had to be removed, a note will indicate the deletion.



UMI U574416

Published by ProQuest LLC 2013. Copyright in the Dissertation held by the Author.

Microform Edition (c) ProQuest LLC.

All rights reserved. This work is protected against unauthorized copying under Title 17, United States Code.

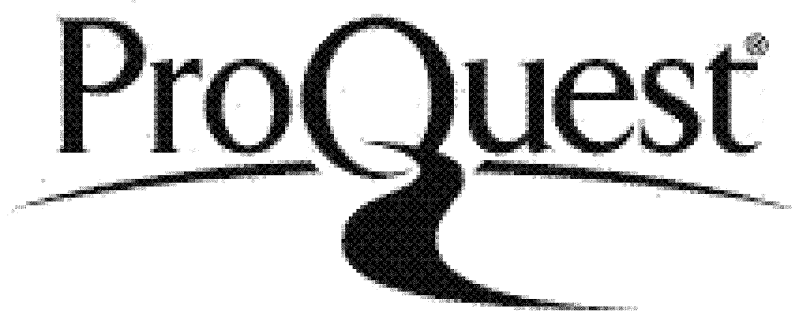

ProQuest LLC

789 East Eisenhower Parkway

P.O. Box 1346

Ann Arbor, MI 48106-1346 
DECLARATION

This work has not been submitted in substance for any other degree or award at this or any other university or place of learning, nor is being submitted concurrently in candidature for any degree or other award.

signed Neil Could any .......... Date 29..... 7 . Loll .

STATEMENT 1

This thesis is being submitted in partial fulfillment of the requirements for the degree of PhD

signed ...Neil .... moulding .......... Date ......29...7. 2011 .

STATEMENT 2

This thesis is the result of my own independent work/investigation, except where otherwise stated.

Other sources are acknowledged by explicit references. The views expressed are my own.

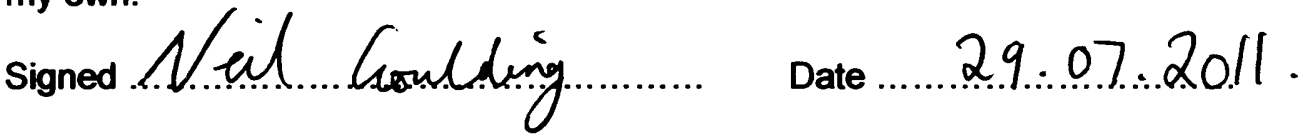

STATEMENT 3

I hereby give consent for my thesis, if accepted, to be available for photocopying and for inter-library loan, and for the title and summary to be made available to outside organisations.

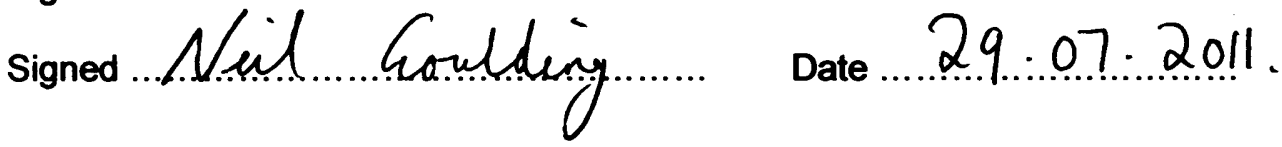

STATEMENT 4: PREVIOUSLY APPROVED BAR ON ACCESS

I hereby give consent for my thesis, if accepted, to be available for photocopying and for inter-library loans after expiry of a bar on access previously approved by the Academic Standards \& Quality Committee.

signed .... Ned God ding .......... Date 29...... 07 . 2011. 


\section{Acknowledgements}

There are several people without whose help and support I could not have finished this thesis.

First and foremost I would like to thank my wife Mary for your incredible amount of patience and giving me the opportunity to follow my dream. To my daughter Lauren, you are my inspiration. Thank you for making me laugh so much. I dedicate this thesis to you both.

I wish to give immense thanks to my supervisor, Professor Russell Davies, for the support and guidance you have given me throughout the course of my studies. It has been a pleasure working with you and you have helped make my PhD an enjoyable and rewarding experience.

I would also like to thank Prof. Ken Walters and Prof. Tim Phillips for agreeing to examine this thesis.

Without the financial support I have received this work would not have been possible. I am extremely grateful for the funding I received from the EPSRC.

Finally, I would like to thank the other staff and fellow students in the School of Mathematics, who were always willing to help whenever I asked. To Stu, Gregor and Heiner, thanks for the banter! 


\section{Summary}

Recovery of the continuous relaxation spectrum of a viscoelastic material from experimental data is an exponentially ill-posed inverse problem. Different methods have been used to deal with the inherent ill-posedness of the problem. Most methods use a discrete relaxation spectrum, but this associates no physical meaning to the spectrum. Empirical models exist for recovering the continuous relaxation spectrum, but there is no theoretical foundation for relating these models directly to the mathematical theory of linear viscoelasticity.

The linchpin of this thesis is in showing that wavelet analysis establishes natural models for the continuous relaxation spectrum. It is shown that there exist wavelet transforms which are intrinsic to the theory of linear viscoelasticity, which give rise to these natural models. In particular, it is shown that the loss modulus is a father wavelet transform of the continuous relaxation spectrum at unit scaling.

A modified version of Calderón-Mallat decomposition is introduced, to express the continuous relaxation spectrum as a sum of continuous wavelets. Atoms are selected from a transformed dictionary of wavelets to fit the storage and loss moduli data, and subsequently form an approximation for the spectrum. Several search and pursuit algorithms are introduced, to select the atoms of the transformed dictionary.

An in-depth account of the wavelet regularization mechanisms acting in this method of continuous relaxation spectrum recovery is given. It is shown that the scaling parameter of the wavelets controls the resolution of the spectrum, whilst the number of basis functions controls the sparsity of the approximation. 


\section{Contents}

$\begin{array}{ll}1 & 7\end{array}$

1.1 Viscoelastic materials. . . . . . . . . . . . . 7

1.2 The continuous relaxation spectrum. . . . . . . . . . 9

1.3 Inverse problems . . . . . . . . . . . . 11

1.3.1 Oscillatory shear experiment . . . . . . . . . . 12

1.4 Complex integral transform relationships between $G^{*}(\omega)$ and $H(\tau) \ldots \ldots \ldots \ldots \ldots \ldots \ldots$

1.4.1 Fredholm linear integral equation of the first kind . . . 16

1.4 .2 Stieltjes Transform . . . . . . . . . . . . . 16

1.5 Fourier analysis and Dirac delta functions . . . . . . . 17

1.6 Previous Work . . . . . . . . . . . . . . 20

1.7 Natural models for the CRS . . . . . . . . . . . . . 22

1.8 Summary of thesis . . . . . . . . . . . . . 24

$2 \quad 27$

2.1 Stieltjes transform inversion. . . . . . . . . . . . . 27

2.2 Double integral inversion formulae . . . . . . . . . . 32

2.2.1 Existence of Moments . . . . . . . . . . . 36

2.3 The Fourier transform of $\operatorname{sech}(x) \ldots \ldots \ldots 38$

2.4 The Fourier transform of $\operatorname{sech}^{2}(x) \ldots \ldots \ldots$. . . . . 40

2.5 Conclusion . . . . . . . . . . . . . . . 42 
3.1 Continuous wavelets . . . . . . . . . . 43

3.1.1 Continuous wavelet transforms . . . . . . . . . 45

3.1.2 Autoconvoluted wavelets . . . . . . . . . . . . 45

3.2 Inversion Formulae . . . . . . . . . . . . . 47

3.3 An illustrative example . . . . . . . . . . . . 50

3.4 FK approximation . . . . . . . . . . . . 51

3.5 Autoconvolutions and cross-pairings. . . . . . . . . 55

3.6 Wavelet frames and Riesz bases. . . . . . . . . . . . 60

3.6.1 Frames . . . . . . . . . . . . . . . 66 60

3.6.2 Hyperbolic and Gaussian wavelet frames . . . . . . 62

3.6.3 Riesz bases. . . . . . . . . . . . . . . . . 64

3.7 MRA and two-scale relations . . . . . . . . . . . . 67

3.8 Choice of primary subspace . . . . . . . . . 73

3.9 Conclusion . . . . . . . . . . . . . . . 74

$\begin{array}{ll}4 & 77\end{array}$

4.1 Real-Time Integrable (RTI) Wavelets . . . . . . . . . . 77

4.2 Model Development for $G^{\prime}$ and $G^{\prime \prime} \ldots \ldots$. . . . . . 81

4.2.1 Scaling function convolutions for $G^{\prime} \ldots \ldots$. . . . 81

4.2.2 Scaling function convolutions for $G^{\prime \prime} \ldots \ldots$. . . . 89

4.2.3 Wavelet convolutions for $G^{\prime} \ldots \ldots$. . . . . . 95

4.2.4 Wavelet convolutions for $G^{\prime \prime} \ldots \ldots$. . . . . 100

4.3 Approximation of the Gaussian . . . . . . . . . . . 104

4.3.1 sech triplet approximation . . . . . . . . . . . 104

4.3.2 Calderón enriched approximation . . . . . . . 106

4.4 Conclusion . . . . . . . . . . . . . . . . . 113

5

5.1 Introduction . . . . . . . . . . . . 115 
5.2 Continuous wavelet dictionaries $\ldots \ldots \ldots \ldots$

5.2 .1 Sparsity in redundant dictionaries . . . . . . . . . 119

5.3 Regularization mechanisms. . . . . . . . . . . 120

5.4 Search Algorithm . . . . . . . . . . . . . . . 121

5.5 PB1 Results . . . . . . . . . . . . . . . . . . . . . . 124

5.5.1 Minimum total curvature as a method for choosing $\sigma . .125$

5.5 .2 Super-resolution . . . . . . . . . . . . . . . 129

5.6 Results from other datasets $\ldots \ldots \ldots . \ldots 135$

5.6 .1 PB2 data . . . . . . . . . . . . 135

5.6 .2 Honerkamp A . . . . . . . . . . . 137

5.6.3 Double Log-Normal Spectrum Approximation . . . . . 139

5.7 Conclusion . . . . . . . . . . . . . . . . . 141

6.1 The $x$ cosech dictionary $\ldots \ldots \ldots \ldots \ldots$

6.1 .1 Wavelet frames . . . . . . . . . . . . 149

6.1 .2 Riesz basis . . . . . . . . . . . . . . 151

6.1 .3 Two-scale relations . . . . . . . . . . . . . 152

6.1 .4 Real-time integrability . . . . . . . . . . 153

6.1.5 Transformed dictionaries . . . . . . . . . . . . 154

6.1.6 Results for real data . . . . . . . . . . . . . 165

6.2 Error terms in the delta sequence method . . . . . . . . . 172

6.3 Wavelet families in linear viscoelasticity . . . . . . . . . 177

6.3.1 Generalized wavelets . . . . . . . . . . . . 177

6.3.2 Generalized wavelet transforms . . . . . . . . . 178

6.3.3 Differentiating wavelet transforms . . . . . . . . 179

6.3.4 $n$-th level reconstruction formulae . . . . . . . . . 181

6.4 Conclusion . . . . . . . . . . . . . . . . . . 182 
7.1 Greedy Algorithms . . . . . . . . . . . . . . 185

7.1.1 Choosing $\Lambda$ by Orthogonal Matching Pursuit (OMP) . 186

7.1 .2 PB1 results . . . . . . . . . . . . 188

7.1 .3 Synthetic data . . . . . . . . . . . . . . 192

7.2 Parity reduction . . . . . . . . . . . . . . . 192

7.3 Density estimation $\ldots \ldots \ldots \ldots \ldots$

7.3 .1 Introduction . . . . . . . . . . . 196

7.3.2 Kernel density approach . . . . . . . . . . . 198

7.3.3 Residual bisection . . . . . . . . . . . . . . . . . 199

7.3.4 Density estimation results for PB1 data . . . . . . 200

7.4 Conclusion . . . . . . . . . . . . . . . 203 


\section{Chapter 1}

\section{Introduction}

\subsection{Viscoelastic materials.}

Most materials which are encountered every day are viscoelastic in nature. Examples include polymers, plastics, foods, oils and paints. Viscous and elastic properties simultaneously exist in these materials. Viscoelasticity is a combination of elastic response and viscous response to applied forces.

Viscoelastic flow is a branch of rheology, which is the science of the deformation and flow of matter. The aim of a rheologist is to obtain constitutive relations linking the stresses to the deformation of a material, which coupled with the kinetics of the flow and conservation laws, provide a complete description of the flow of that material and may then be applied to the solution of engineering problems such as those involved in the manufacturing of products mentioned above.

A large amount of rheological study has been conducted in the area of polymeric materials, encouraged by the significant use of these materials in engineering and manufacturing. The viscoelastic behaviour of polymers arises from the movement of thread-like, flexible long-chain macromolecules which occupy a volume considerably greater than atomic dimensions. As 
the material undergoes deformation, internal forces develop as a result of molecular changes in configuration, involving rearrangements on different scales [79]:

1. Long-range contour rearrangements (slow).

2. Local level rearrangements, e.g. kinks (more rapid).

3. Reorientation of bonds on the chain backbone on the atomic scale.

Consequently, the stresses are influenced on several timescales, which leads to complex behaviour as the rearrangements take place at different time scales.

The area of viscoelastic theory that is studied in this thesis is the linear viscoelastic regime. This linear study is helpful in clarifying the molecular structure of materials, and the molecular weight distribution can be calculated from linear viscoelastic data. Methods are connected with reptation based mixing rules (Mead [56], Honerkamp et al. [72], Bailly et al. [75]). It is also a useful basis to proceed to the theory of non-linear viscoelasticity. In fact the memory function of the linear regime can be used in the modelling of non-linear deformations.

The theory of linear viscoelasticity is concerned with a restricted class of flows which have very small strains and strain rates. This theory characterizes the materials by describing how they flow, or deform over time, under a given stress or by the force needed to produce a certain strain. For example, in a shear flow, stress is the force per unit area in the direction of shear, while the strain is a measure of deformation of the material.

The field of rheometry (Walters [78]) is the area of rheology concerned with measurement of the characteristic functions of viscoelastic materials, which relate the stress and strain within the sample material, by experimental methods. The behaviour of viscoelastic materials in simple (rheometrical) flow geometries, such as steady shear flow or small amplitude oscillatory shear 
is obtained with the intention of utilizing this information to predict the flow in more complex situations. Often it is necessary to ensure the deformations are sufficiently small that a linear approximation can be employed; the linear viscoelastic functions being thus determined can be used in non-linear constitutive equations combined with the stress equations of motion and continuity conditions to model industrial processes involving viscoelastic materials by computational methods. Another motivation of rheometry is to relate the molecular structure to the material behaviour, interpreting the motion of the polymer chains in terms of the relaxation spectrum. More details on linear viscoelasticity can be found in Ferry [33], Barnes et al. [11] and Gross [39].

\subsection{The continuous relaxation spectrum.}

An important quantity in the characterization of linear viscoelastic materials is the relaxation spectrum. The most fundamental use in determining a materials relaxation spectrum is to gain some deeper insight in to the relaxation mechanisms of the material. It reflects the molecular processes occurring at a certain time scale. These depend on temperature and pressure, but in this thesis I shall assume that all data sets used have been measured at constant temperature and pressure. Among the processes being reflected in the spectrum is the molecular structure in a broad sense, characterized by molar mass distribution and long-chain branching architecture. A rheological measurement can be understood as the response of the structure, represented by the spectrum, to the mechanical stimulation [70].

The response of a linear viscoelastic material to small strain excitation is determined completely by its relaxation spectrum. In an incompressible shear deformation, Boltzmann's general linear integral model for viscoelastic 
materials [17] relates the stress $\sigma(t)$ to the strain $\gamma(t)$ in the form

$$
\sigma(t)=\int_{-\infty}^{t} G\left(t-t^{\prime}\right) \dot{\gamma}\left(t^{\prime}\right) d t^{\prime}
$$

where $G(t)$ is a monotonically decreasing relaxation function. The principle of fading memory [66] demands that the first derivative $\frac{d G}{d t}$ is monotonically increasing, where the memory function $M(t)$ is related to the relaxation function $G(t)$ by

$$
M(t)=-\frac{d G(t)}{d t}
$$

Bernstein's theorem [15] states that $G(t)$ is completely monotone (i.e. successive derivatives of all orders are alternately monotonically increasing and decreasing):

$$
(-1)^{n} \frac{d^{n} G(t)}{d t^{n}} \geq 0, \quad n \geq 0, \quad t>0
$$

if and only if $G(t)$ is the Laplace transform of a positive measure $\mu$ on $(0, \infty)$, i.e.

$$
G(t)=\int_{0}^{\infty} e^{-t s} d \mu(s)
$$

where the integral converges for all $t>0$. Under this constraint $G(t)$ may be written in the form

$$
G(t)=G_{e}+\int_{0}^{\infty} H(\tau) e^{-\frac{t}{\tau}} \frac{d \tau}{\tau}
$$

[11], where $G_{e}$ is a material constant, given by

$$
G_{e}=\lim _{t \rightarrow \infty} G(t),
$$

and $H(\tau)$ is an un-normalized non-negative density function associated with a continuous range of relaxation times $\tau . H(\tau) d \tau$ is defined as the viscosity 
associated with the relaxation time between $\tau$ and $\tau+d \tau . H(\tau)$ is known as the continuous relaxation spectrum and the total viscosity $\eta$ of the material is given by

$$
\eta=\int_{0}^{\infty} H(\tau) d \tau<\infty
$$

The term $G_{e}$ is included to incorporate viscoelastic solid behaviour. This represents the fact that, for a viscoelastic solid under constant strain, the stress will never relax to zero. Conversely, $G_{e}=0$ for viscoelastic fluids.

\subsection{Inverse problems}

Relaxation spectra can only be determined indirectly, that is by performing an experiment, and solving one or more inverse problems using measured data from the experiment. Inverse problems arise in a variety of important applications in science and industry. Many examples are given by Wing and Zahrt [80], Groetsch [38] and Engl et al. [32]. Unfortunately, a small amount of noise in the data can lead to enormous errors in the estimates. This instability phenomenon is a result of ill-posedness. Mathematical techniques that can cope with this type of problem are called regularization methods (see [32]).

An inverse problem does not necessarily have a solution or even a unique solution. Furthermore, if a solution exists, it does not continuously depend on the data, i.e. it is unstable. This leads to the following definition of wellposedness for identification problems, which was first given by Hadamard [40]:

Definition 1.2.1 Let $X$ and $Y$ be two topological spaces and $K: X \rightarrow Y$ be a mapping from $X$ into $Y$. According to Hadamard the operator equation

$$
K x=y, \quad x \in D \subset X, y \in Y,
$$


is said to be well-posed, if the following three conditions hold:

1. For every $y \in Y$ there exists at least one $x \in D$ satisfying $K x=y$ (existence).

2. The element $x$ satisfying that $K x=y$ is uniquely determined in $D$ (uniqueness).

3. The solution $x$ depends continuously on the right hand side $y$ (stability).

If one of these conditions is not satisfied, then the problem (1.3.1) is called ill-posed in the sense of Hadamard.

If equation (1.3.1) is well-posed, then $K$ has a well-defined, continuous inverse operator $K^{-1}$. Direct problems are usually well-posed whereas the nature of inverse problems often (but not always) lead to ill-posedness as a characteristic property. Further explanations, theories and examples on inverse and ill-posed problems can be found in [38].

\subsubsection{Oscillatory shear experiment}

Despite the abundance of new techniques proposed in recent times, the simple small-amplitude oscillatory shear experiment is still one of the popular techniques performed for determining the linear viscoelastic functions $[71,78]$. This experiment enables the measurement of the real and imaginary parts of a complex modulus $G^{*}(\omega)$ as a function of angular frequency $\omega$. In the experiment, the viscoelastic material is positioned between two plates, with one plate rotating relative to the other (Fig. 1.1). An oscillatory strain is induced in the material under investigation at a specific, fixed frequency $\omega_{k}$ and the resulting stress is measured, which is also of the same frequency, from which the phase angle $\delta$ and amplitude ratio can be used to calculate $G^{\prime}\left(\omega_{k}\right)$ and $G^{\prime \prime}\left(\omega_{k}\right)$ at that particular frequency. The experiment is then repeated 
at the next frequency, and so on until the desired range of frequencies has been covered. Such experiments are termed dynamic since the excitation is a continually varying function of time.

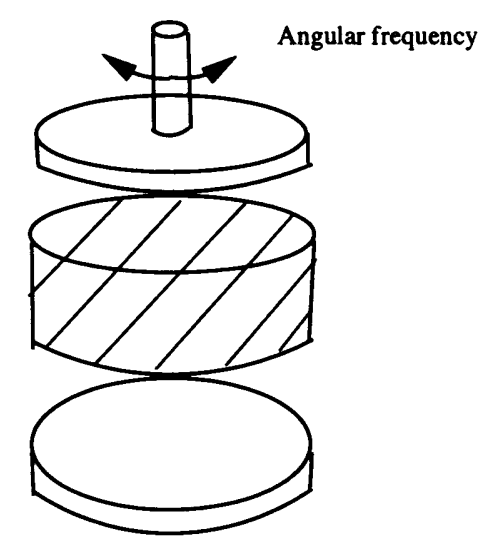

Figure 1.1: Oscillatory shear flow.

For an angular frequency $\omega$ and an applied strain

$$
\gamma(t)=\left\{\begin{array}{l}
\gamma_{0} e^{i \omega t} \quad \text { if } t \geq 0 \\
0 \quad \text { if } t<0
\end{array}\right.
$$

where $\gamma_{0}$ is a strain amplitude small enough for the linearity assumption to be justified, equation (1.2.1) can be expressed as

$$
\begin{aligned}
\sigma(t) & =\int_{-\infty}^{t}\left(G_{e}+\left[G\left(t-t^{\prime}\right)-G_{e}\right]\right) \dot{\gamma}\left(t^{\prime}\right) d t^{\prime} \\
& =G_{e} \int_{-\infty}^{t} \dot{\gamma}\left(t^{\prime}\right) d t^{\prime}+\int_{-\infty}^{t}\left[G\left(t-t^{\prime}\right)-G_{e}\right] \dot{\gamma}\left(t^{\prime}\right) d t^{\prime} \\
& =G_{e} \gamma(t)+i \omega \gamma_{0} \int_{-\infty}^{t}\left[G\left(t-t^{\prime}\right)-G_{e}\right] e^{i \omega t^{\prime}} d t^{\prime} \\
& =G_{e} \gamma(t)+i \omega \int_{0}^{\infty}\left[G(s)-G_{e}\right] e^{-i \omega s} d s \gamma_{0} e^{i \omega t} \\
& =G^{*}(\omega) \gamma(t),
\end{aligned}
$$


where $G^{*}(\omega)$ is a complex shear modulus given by

$$
G^{*}(\omega)=G_{e}+i \omega \int_{0}^{\infty}\left[G\left(t^{\prime}\right)-G_{e}\right] e^{-i \omega t^{\prime}} d t^{\prime} .
$$

Substituting equation (1.2.5) into (1.3.4) allows $G^{*}(\omega)$ to be related to the continuous relaxation spectrum by

$$
\begin{aligned}
G^{*}(\omega) & =G_{e}+i \omega \int_{0}^{\infty} \int_{0}^{\infty} H(\tau) e^{-\frac{t^{\prime}}{\tau}} \frac{d \tau}{\tau} e^{-i \omega t^{\prime}} d t^{\prime} \\
& =G_{e}+i \omega \int_{0}^{\infty} \frac{H(\tau)}{\tau} \int_{0}^{\infty} \exp \left[-\left(\frac{1+i \omega \tau}{\tau}\right) t^{\prime}\right] d t^{\prime} d \tau \\
& =G_{e}+\int_{0}^{\infty} \frac{i \omega \tau}{1+i \omega \tau} \frac{H(\tau)}{\tau} d \tau
\end{aligned}
$$

From equation (1.3.5), $G^{*}(\omega)$ can be split into real and imaginary parts as follows

$$
\begin{aligned}
G^{*}(\omega) & =G_{e}+\int_{0}^{\infty} \frac{\omega^{2} \tau^{2}}{1+\omega^{2} \tau^{2}} \frac{H(\tau)}{\tau} d \tau+i \int_{0}^{\infty} \frac{\omega \tau}{1+\omega^{2} \tau^{2}} \frac{H(\tau)}{\tau} d \tau \\
& =G^{\prime}(\omega)+i G^{\prime \prime}(\omega)
\end{aligned}
$$

where

$$
G^{\prime}(\omega)=G_{e}+\int_{0}^{\infty} \frac{\omega^{2} \tau^{2}}{1+\omega^{2} \tau^{2}} \frac{H(\tau)}{\tau} d \tau
$$

and

$$
G^{\prime \prime}(\omega)=\int_{0}^{\infty} \frac{\omega \tau}{1+\omega^{2} \tau^{2}} \frac{H(\tau)}{\tau} d \tau
$$

are known respectively as the storage and loss moduli. The storage modulus is often thought of characterizing the elastic component of the response and hence the amount of energy stored per cycle of deformation, and the loss modulus as describing the amount of energy dissipated per cycle by the viscous component.

For the rest of this thesis it will be assumed that $G_{e}=0$. 
Fig. 1.2 is an example of complex data quoted by Honerkamp [43] from an oscillatory shear experiment performed on a polybutadiene polymer blend, where $G_{e}=0$. The storage moduli is plotted in red, the loss moduli is plotted in blue and $x=\ln (\omega)$. This data will be referred to in this thesis as PB1 data.

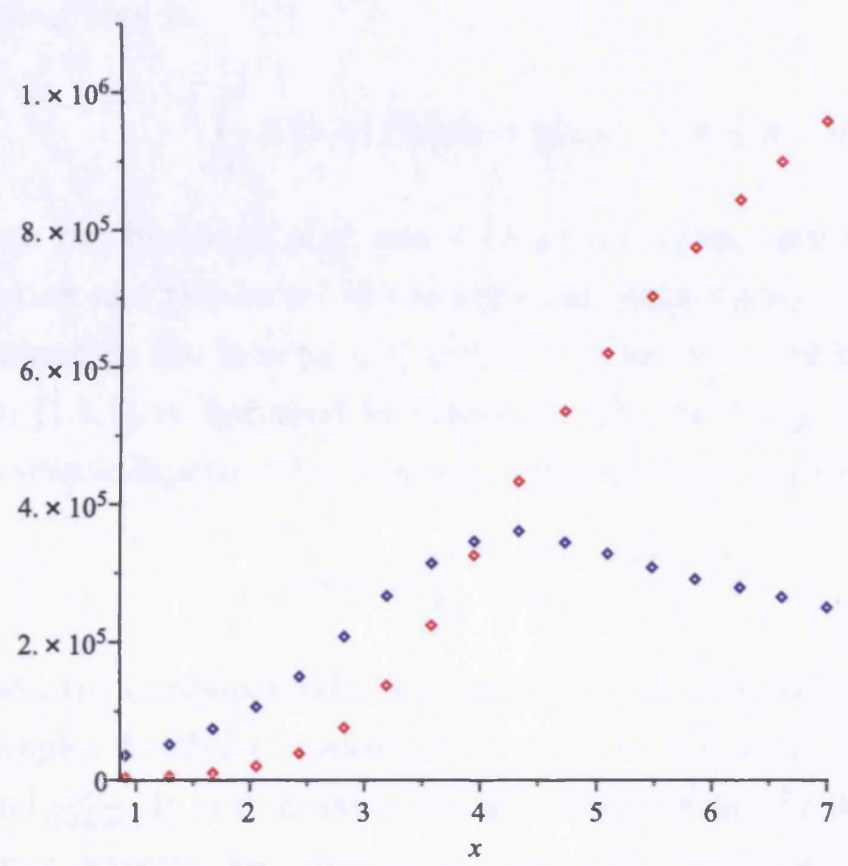

Figure 1.2: $G^{\prime}(\omega)$ data (red) and $G^{\prime \prime}(\omega)$ data (blue), obtained from an oscillatory shear experiment performed on a polybutadiene polymer blend, where $x=\ln \omega$. 


\subsection{Complex integral transform relationships between $G^{*}(\omega)$ and $H(\tau)$}

\subsubsection{Fredholm linear integral equation of the first kind}

Definition 1.4.1. The general form of Fredholm linear integral equation of the first kind is

$$
\begin{gathered}
\int^{b} K(r u f(u) d u=g(x), \quad c \leq x \leq d \\
\int_{a}^{o} K(x, y) f(y) d y=g(x),
\end{gathered}
$$

where the functions $g(x)$ and $K(x, y)$ are given, and are called the datafunction and the kernel of the equation, respectively. $f(y)$ needs to be determined on the interval $a \leq y \leq b$. The theory concerning integrals of the form (1.4.1) is discussed by Groetsch [38] and Wing and Zahrt [80]. For viscoelastic liquids, $G^{*}(\omega)$ can be expressed from equation (1.3.5) as

$$
G^{*}(\omega)=\int_{0}^{\infty} \frac{i \omega \tau}{1+i \omega \tau} \frac{H(\tau)}{\tau} d \tau
$$

Hence, the complex modulus is related to the relaxation spectrum $H(\tau)$ by a complex Fredholm integral equation of the first kind, of product type, with kernel $\frac{i \omega \tau}{1+i \omega \tau}$. In real measure this kernel is dilationally invariant; in the logarithmic measure the kernel is translationally invariant, and is a convolution kernel.

\subsubsection{Stieltjes Transform}

Under the transformation $z=i \omega^{-1}$, we obtain

$$
g(z) \equiv G^{*}\left(i z^{-1}\right)=\int_{0}^{\infty} \frac{H(\tau)}{\tau-z} d \tau
$$


which relates the complex modulus to the continuous relaxation spectrum by a complex Stieltjes transform. Under simple regularity conditions on $H(\tau)$, for complex values of $z, g(z)$ is analytic in the upper and lower half planes, and exists as a Cauchy principal value integral when $z$ is real. Furthermore, the Stieltjes-Perron inversion formula [42] for (1.4.3) is

$$
\begin{aligned}
H(\tau) & =\frac{1}{2 \pi i} \lim _{\epsilon \rightarrow 0+}[g(\tau+i \epsilon)-g(\tau-i \epsilon)] \\
& =\frac{1}{\pi} \lim _{\epsilon \rightarrow 0+} \Im[g(\tau+i \epsilon)] \\
& =\frac{1}{\pi} \lim _{\epsilon \rightarrow 0+} \Im\left[G^{*}\left(i \tau^{-1}+\epsilon\right)\right] .
\end{aligned}
$$

When $z$ is real valued, $g(z)$ is a Hilbert transform of $H(\tau)$, and its inverse is given by

$$
H(\tau)=\frac{1}{\pi} g(\tau)=\frac{1}{\pi} G^{*}\left(i \tau^{-1}\right)
$$

where $g(\tau)$ and $G^{*}\left(i \tau^{-1}\right)$ are defined in terms of principle value integrals. The inversion here is well-posed. However, it is impossible to calculate the values of $G^{*}$ on the imaginary axis knowing only sampled values on the real axis.

An alternative inversion formula is given by the Stieltjes-Perron formula (1.4.6). The inversion here is equivalent to finding the limit on the imaginary axis of a function which is analytic in the right-hand half plane. This problem is ill-posed. In fact, when $z$ is pure imaginary, it will be shown in Chapter 2 that Stieltjes transform inversion is exponentially ill-posed.

\subsection{Fourier analysis and Dirac delta func- tions}

Some knowledge of Fourier analysis and Dirac delta functions will be required throughout this thesis. In this section I will collect together the 
definitions which are needed.

Definition 1.5.1. (The Fourier transform) The Fourier transform $\widehat{f}(p)$ of $f(x)$ in $L^{2}(\mathbb{R})$ is defined by (see [53])

$$
\widehat{f}(p)=\int_{-\infty}^{\infty} f(x) e^{-i p x} d x
$$

The inverse Fourier transform is then given by

$$
f(x)=\frac{1}{2 \pi} \int_{-\infty}^{\infty} \widehat{f}(p) e^{i p x} d p \quad\left(=[\widehat{f}(p)]^{\vee}(x)\right) .
$$

If $f \in L^{2}(\mathbb{R})$ then so is $\widehat{f}$. If both $f$ and $\widehat{f}$ belong to $L^{1}(\mathbb{R})$ then both (1.5.1) and (1.5.2) are valid. An important attribute of the Fourier transform is that the total area of the graph of a function $f(x)$ is equal to its Fourier transform evaluated at $p=0$ :

$$
\int_{-\infty}^{\infty} f(x) d x=\widehat{f}(0)
$$

Definition 1.5.2. If $f_{1} \in L^{2}(\mathbb{R})$ and $f_{2} \in L^{2}(\mathbb{R})$, then the convolution of the two functions $f_{1}(x)$ and $f_{2}(x)$ is defined by

$$
\left(f_{1} \star f_{2}\right)(x)=\int_{-\infty}^{\infty} f_{1}(x-y) f_{2}(y) d y .
$$

Theorem 1.5.1. (Convolution theorem) The Fourier transform of the convolution of two functions is the product of their Fourier transforms, i.e.

$$
\widehat{f_{1} \star f_{2}}(p)=\widehat{f}_{1}(p) \widehat{f}_{2}(p)
$$

Table 1.1 gives a list of some of the important properties of the Fourier transform that will be used throughout this thesis [53].

Definition 1.5.3. (The Dirac delta function) The Dirac delta function $\delta(t)$ can be obtained by scaling an integrable function $g$ which satisfies

$$
\int_{-\infty}^{\infty} g(t) d t=1
$$




\begin{tabular}{|c|c|c|}
\hline Property & Function & Fourier transform \\
\hline & $f(t)$ & $\widehat{f}(p)$ \\
\hline Inverse & $\widehat{f}(t)$ & $2 \pi f(-p)$ \\
\hline Convolution & $\left(f_{1} \star f_{2}\right)(t)$ & $\widehat{f}_{1}(p) \widehat{f}_{2}(p)$ \\
\hline Multiplication & $f_{1}(t) f_{2}(t)$ & $\frac{1}{2 \pi}\left(\widehat{f}_{1} \star \widehat{f}_{2}\right)(p)$ \\
\hline Translation & $f\left(t-t_{0}\right)$ & $e^{-i t_{0} p} \widehat{f}(p)$ \\
\hline Modulation & $e^{-i p_{0} t} f(t)$ & $\widehat{f}\left(p-p_{0}\right)$ \\
\hline Scaling & $f\left(\frac{t}{s}\right)$ & $|s| \widehat{f}(s p)$ \\
\hline Time derivatives & $f^{(n)}(t)$ & $(i p)^{n} \widehat{f}(p)$ \\
\hline Frequency derivatives & $(-i t)^{n} f(t)$ & $\widehat{f}^{(n)}(p)$ \\
\hline Complex conjugate & $f^{*}(t)$ & $\widehat{f}^{*}(-p)$ \\
\hline Hermitian symmetry & $f(t) \in \mathbb{R}$ & $\widehat{f}(-p)=\widehat{f}^{*}(p)$ \\
\hline
\end{tabular}

Table 1.1: Properties of the Fourier transform [51].

Let

$$
g_{s}(t)=\frac{1}{s} g\left(\frac{t}{s}\right)
$$

A Dirac delta function can then be defined as the limit

$$
\delta(t)=\lim _{s \rightarrow 0} g_{s}(t),
$$

with the property that for any continuous function $f$ at the origin

$$
\int_{-\infty}^{\infty} \delta(t) f(t) d t=f(0)
$$

since

$$
\lim _{s \rightarrow 0} \int_{-\infty}^{\infty} g_{s}(t) f(t) d t=\lim _{s \rightarrow 0} \int_{-\infty}^{\infty} g(u) f(s u) d u .
$$

The property (1.5.9) is only a symbolic notation of the fact that when a Dirac delta is applied to a continuous function $f$, it returns its value at $t=0$. The 
symbolic integral over a Dirac is useful notation because it has the same properties as a usual integral, including change of variables and integration by parts.

A translated Dirac $\delta_{\tau}=\delta(t-\tau)$ has a mass centred at $\tau$ and

$$
\int_{-\infty}^{\infty} f(t) \delta(u-t) d t=f(u)
$$

This means that

$$
f \star \delta=f(u)
$$

The Fourier transform of $\delta$ takes the value 1 for all $p$, i.e.

$$
\widehat{\delta}(p)=\int_{-\infty}^{\infty} \delta(t) e^{-i p t} d t=1
$$

\subsection{Previous Work}

For a one mode Maxwell model the relaxation spectrum is discrete and is given by

$$
H(\tau)=\eta_{1} \delta\left(\tau-\tau_{1}\right)
$$

where $\delta$ is a Dirac measure. The stress is then related to the strain-rate by

$$
\sigma(t)=\frac{\eta_{1}}{\tau_{1}} \int_{-\infty}^{t} \exp \left[-\frac{\left(t-t^{\prime}\right)}{\tau_{1}}\right] \dot{\gamma}\left(t^{\prime}\right) d t^{\prime}
$$

A Maxwell element is a model for primitive viscoelastic behaviour, where a spring and dashpot are placed in series. A generalized Maxwell model with a discrete spectrum of viscosities and relaxation times can be obtained by placing simple Maxwell elements in parallel. Each element corresponds to a different relaxation mechanism in the fluid. 
Most previous work in relaxation spectrum recovery involves determining discrete spectra, by fitting discrete models to data. The $n$ mode Maxwell model has a discrete relaxation spectrum

$$
H(\tau)=\sum_{n} \eta_{n} \delta\left(\tau-\tau_{n}\right)
$$

The corresponding storage and loss moduli are given by

$$
G^{\prime}(\omega)=\sum_{n} \frac{\eta_{n}}{\tau_{n}} \frac{\omega^{2} \tau_{n}^{2}}{1+\omega^{2} \tau_{n}^{2}}, \quad G^{\prime \prime}(\omega)=\sum_{n} \frac{\eta_{n}}{\tau_{n}} \frac{\omega \tau_{n}}{1+\omega^{2} \tau_{n}^{2}}
$$

Different ways have been chosen to deal with the inherent ill-posedness of the problem. Methods for determining discrete spectra include the use of nonlinear regression [13,61], Tikhonov regularisation [43], maximum entropy regularisation [30], sampling localisation [5,49] and Prony series [20].

Different methods can give rise to different discrete spectra. There is no unique discrete spectrum for any given material. As quoted by A. Ya Malkin [50]: "The discrete relaxation spectrum is just a convenient way of representing experimental data... It has no basic physical meaning". A further quote from Chow and Zukoski [23,24] states: "No line spectrum - produced by whatever method - is ever the true spectrum". Also, from Dealy and Larson [29]: "Whenever an empirical expression is fitted to experimental data, information is lost and/or error is introduced. Every such manipulation of data therefore involves a degradation of the information contained in the original data. ... the use of a discrete spectrum involves the use of an empirical equation to fit data. The resulting constants have no physical significance, and the resulting function will have local features that are artifacts of the model and do not reflect the structure of the polymer. This can cause trouble, for example if this function is used to infer the molecular weight distribution. For such a purpose, it may thus be preferable to work with a continuous spectrum function such as $H(\tau)$ ". 
It is an inherent premise in Boltzmann formulation of linear viscoelasticity that, for shear deformations at fixed temperature and pressure, a viscoelastic material has a unique continuous relaxation spectrum associated with it. Empirical models for representing the continuous spectrum have been suggested by Winter [14] (who attempts to derive an approximate relation between continuous and discrete relaxation time spectra), Anderssen [7] (who uses Kohlrausch (stretched exponential) functions), Bailly and Stadler [70] (who use piecewise cubic Hermite splines) and Malkin [52] (whose central idea is that the parameters of the continuous spectrum can be unambiguously determined from the experimentally found integral characteristics (moments) of the viscoelastic behavior of the material: the viscosity, normal stress coefficient, and area under the relaxation curve after the cessation of steady flow).

All the above continuous relaxation spectrum models fall into the class of parametric curve fitting models. There is no theoretical foundation for relating these models directly to the mathematical theory of linear viscoelasticity. The aim of this thesis is to show that wavelet analysis establishes natural models for the continuous relaxation spectrum. In particular, in Chapter 2 it is shown that there exist wavelet transforms which are intrinsic to the theory of linear viscoelasticity, which give rise to these natural models.

\subsection{Natural models for the continuous relax- ation spectrum}

Recently, the subject of wavelet analysis has drawn much attention from both mathematicians and engineers alike. Some view wavelets as a new basis for representing functions, some consider it as a technique for time-frequency analysis, and others think of it as a new mathematical subject. In fact, all of 
them are right, since wavelets is a versatile tool with very rich mathematical content and great potential for applications. Analogous to Fourier analysis, there are also two important mathematical entities in wavelet analysis: the integral wavelet transform and the wavelet series [25].

This thesis is concerned with continuous wavelets. The mother wavelet $\psi$ has zero area

$$
\int_{-\infty}^{\infty} \psi(x) d x=0
$$

and satisfies strict admissability and regularity criteria

$$
0<C_{\psi}=\int_{0}^{\infty} \frac{\widehat{\psi}(p)^{2}}{p} d p<\infty
$$

Equation (1.7.2) means that $\psi$ admits a scaling function. The father wavelet (or scaling function), $\phi$, was introduced by Mallat [53]. $\phi$ has non-zero area and is defined by its Fourier transform

$$
\widehat{\phi}(p)=\left[\int_{p}^{\infty} \frac{\widehat{\psi}(\xi)^{2}}{\xi} d \xi\right]^{\frac{1}{2}}
$$

At different time locations, and different time scales (resolution with respect to time) the mother and father wavelets generate a family of wavelets

$$
\psi_{s, t}(x)=\frac{1}{\sqrt{s}} \psi\left(\frac{x-t}{s}\right), \quad \phi_{s, t}(x)=\frac{1}{\sqrt{s}} \phi\left(\frac{x-t}{s}\right) .
$$

The (continuous) wavelet transform of $h \in L^{2}(\mathbb{R})$ at the time $t$ and scale $s$ is defined by the convolution product

$$
\begin{aligned}
{[W h](s, t) } & =\left(h \star \psi_{s}\right)(t), \\
\text { where } \quad \psi_{s}(x) & =\psi_{s, 0}(x)=\frac{1}{\sqrt{s}} \psi\left(\frac{x}{s}\right) .
\end{aligned}
$$

I define a father wavelet transform of $h \in L^{2}(\mathbb{R})$ by

$$
\begin{aligned}
{[V h](s, t) } & =\left(h \star \phi_{s}\right)(t), \\
\text { where } \quad \phi_{s}(x) & =\phi_{s, 0}(x)=\frac{1}{\sqrt{s}} \phi\left(\frac{x}{s}\right) .
\end{aligned}
$$


It will be shown in Chapter 3 that, under a transformation of variables, the loss modulus is a special type of wavelet transform. Recovery of the continuous relaxation spectrum consequently involves the inversion of wavelet transforms and it will be shown that the continuous relaxation spectrum can be expressed as a sum of mother and father wavelets.

\subsection{Summary of thesis}

In this first chapter I have introduced some of the concepts involved in materials characterization. One of the ways of characterizing a viscoelastic material is through its relaxation spectrum and it has been explained that recovery of the continuous relaxation spectrum is an ill-posed inverse problem.

In Chapter 2 it is proved that recovery of the continuous relaxation spectrum is exponentially ill-posed. Several double integral inversion formulae are derived for relating moments of the relaxation spectrum to data involving $\omega^{m} G^{*}(\omega)$.

The concept that wavelet transforms occur naturally in the theory of linear viscoelasticity is introduced in Chapter 3. The Calderón-Mallat decomposition of the identity [53] is introduced, which gives an inversion formula for reconstructing the continuous relaxation spectrum from its wavelet transforms, and the spectrum is expressed as a wavelet series. I introduce a modified version of Calderón-Mallat decomposition, using father and mother wavelets from different families, and show that these cross-paired wavelets form an essential multiresolution analysis.

In Chapter 4, the concept of real-time integrability is introduced. Natural models for $G^{\prime}(\omega)$ and $G^{\prime \prime}(\omega)$ are derived analytically, using residue calculus. In this chapter, I illustrate how well my models can recover a Gaussian spectrum, from a direct method and from using synthetic data. 
In Chapter 5, I introduce wavelet dictionaries and the regularization mechanisms acting in my method of continuous relaxation spectrum recovery. The continuous relaxation spectrum is predicted from two sets of real data, obtained from an oscillatory shear experiment performed on two polybutadiene polymer blends. In both cases a simple search algorithm is implemented, which is also introduced in this chapter.

Chapter 6 introduces other dictionaries that can be used to construct the continuous relaxation spectrum. The main purpose of this chapter is to give consistency of results. Also in this chapter I explore families of wavelet transforms that occur naturally in the theory of linear viscoelasticity, and I give possible mother and father wavelet pairs that can be used in the recovery process. Furthermore, error terms in the delta sequence method of relaxation spectrum recovery are derived in this chapter.

Chapter 7 explores other more advanced search and pursuit algorithms, for fitting the dynamic data to the models. Orthogonal Matching Pursuit (OMP), parity reduction, density estimation and residual bisection are all introduced in this chapter. 
CHAPTER 1. 


\section{Chapter 2}

\section{Fourier Analysis and Stieltjes Transform Inversion.}

\subsection{Stieltjes transform inversion.}

In Chapter 1 it was shown that the complex shear modulus is related to the continuous relaxation spectrum by the following complex Stieltjes transform

$$
G^{*}(\omega)=\int_{0}^{\infty} \frac{i \omega \tau}{1+i \omega \tau} H(\tau) \frac{d \tau}{\tau}=\int_{0}^{\infty} \frac{H(\tau)}{\tau-z} d \tau, \quad z=i \omega^{-1}
$$

I will now show that recovery of the continuous relaxation spectrum is an exponentially ill-posed inverse problem.

The real part of the kernel of (2.1.1) can be written as

$$
\frac{\omega^{2} \tau^{2}}{1+\omega^{2} \tau^{2}}=\frac{1}{2}[1+\tanh (\ln (\omega \tau))]
$$

while the imaginary part of the kernel of (2.1.1) is

$$
\frac{\omega \tau}{1+\omega^{2} \tau^{2}}=\frac{1}{2} \operatorname{sech}(\ln (\omega \tau))
$$


Consequently, we obtain the following expressions for the real and imaginary parts of the complex modulus, known as the storage and loss moduli respectively

$$
\begin{aligned}
& G^{\prime}(\omega)=\frac{1}{2} \int_{0}^{\infty}[1+\tanh (\ln (\omega \tau))] \frac{H(\tau)}{\tau} d \tau, \\
& G^{\prime \prime}(\omega)=\frac{1}{2} \int_{0}^{\infty} \operatorname{sech}(\ln (\omega \tau)) \frac{H(\tau)}{\tau} d \tau .
\end{aligned}
$$

Using the substitutions

$$
H(\tau)=h(t), \quad G^{\prime}(\omega)=\frac{1}{2} g_{1}(x), \quad G^{\prime \prime}(\omega)=\frac{1}{2} g_{2}(x)
$$

with

$$
x=\ln \omega, \quad t=-\ln \tau,
$$

the storage and loss moduli may then be written in the form

$$
\begin{aligned}
& g_{1}(x)=\int_{-\infty}^{\infty}[1+\tanh (x-t)] h(t) d t, \\
& g_{2}(x)=\int_{-\infty}^{\infty} \operatorname{sech}(x-t) h(t) d t .
\end{aligned}
$$

From definition (1.5.2), we note that these are a pair of convolution equations. Consequently, the real and imaginary parts of the complex modulus can be expressed in convolution form as

$$
\begin{aligned}
& g_{1}(x)=[1+\tanh (x)] \star h(x) \\
& g_{2}(x)=\operatorname{sech}(x) \star h(x) .
\end{aligned}
$$

I will assume throughout this thesis that $h(x) \in L^{2}(\mathbb{R})$, which then implies that its Fourier transform $\widehat{h}(p)$ exists.

Since $\operatorname{sech}(x) \in L^{2}(\mathbb{R})$, it follows from the convolution theorem for Fourier transforms (1.5.5) that

$$
\widehat{g_{2}}(p)=[\operatorname{sech}(x)]^{\wedge}(p) \widehat{h}(p) .
$$


The Fourier transform of $\operatorname{sech}(x)$ is calculated via residue calculus, in section 2.3 , where it is found that

$$
\begin{aligned}
{[\operatorname{sech}(x)]^{\wedge}(p) } & =\pi \operatorname{sech}\left(\frac{\pi}{2} p\right) \\
\therefore \widehat{g_{2}}(p) & =\pi \operatorname{sech}\left(\frac{\pi}{2} p\right) \widehat{h}(p),
\end{aligned}
$$

where $\widehat{g_{2}}(p) \in L^{2}(\mathbb{R})$ if $h \in L^{2}(\mathbb{R})$.

The function $\tanh (x)$ is in neither $L^{1}(\mathbb{R})$ or $L^{2}(\mathbb{R})$, but has a derivative

$$
g_{3}(x)=\operatorname{sech}^{2}(x)
$$

which is in both $L^{1}(\mathbb{R})$ and $L^{2}(\mathbb{R})$. The Fourier transform of $\tanh (x)$ does not exist in the classical sense, but can be defined in a distributional sense (see Kanwal [46]) by

$$
[\tanh (x)]^{\wedge}(p)=(i p)^{-1} \widehat{g_{3}}(p) .
$$

Let $S_{\frac{\pi}{2}}$ be a space of test functions defined as follows. If $\phi \in S_{\frac{\pi}{2}}$ then

1. $\phi(x) \in \mathbb{C}^{\infty}(\mathbb{R})$.

2. $\forall n \geq 0, \phi^{(n)}(x)$ decays faster than $\operatorname{sech}\left(\frac{\pi}{2} x\right)$ as $x \rightarrow \pm \infty$.

3. There exists a null sequence of test functions $\phi_{m}(x) \in S_{\frac{\pi}{2}}$ such that

$$
\lim _{m \rightarrow \infty} \phi_{m}(x)=0 \text {. }
$$

Two examples of sequences of test functions that satisfy the above properties are the sequence of Gaussian functions

$$
\phi_{m}(x)=\frac{1}{m} e^{-\frac{(m x)^{2}}{2}}, \quad m=1,2, \ldots
$$

and the sequence of sech functions

$$
\phi_{m}(x)=\frac{1}{m} \operatorname{sech}(m \lambda x) \quad \lambda>\frac{\pi}{2}, \quad m=1,2, \ldots
$$


Consider the inner product

$$
\langle\bullet, \phi(p)\rangle=\int_{-\infty}^{\infty} \bullet \phi(p) \tanh \left(\frac{\pi}{2} p\right) d p
$$

I then obtain

$$
\begin{aligned}
\left\langle[\tanh (x)]^{\wedge}(p), \phi(p)\right\rangle & =\left\langle(i p)^{-1} \widehat{g_{3}}(p), \phi(p)\right\rangle \\
& =-i \pi \int_{-\infty}^{\infty} \operatorname{cosech}\left(\frac{\pi}{2} p\right) \phi(p) \tanh \left(\frac{\pi}{2} p\right) d p \quad \forall \phi \in S_{\frac{\pi}{2}},
\end{aligned}
$$

where I have used the fact that

$$
\left[\operatorname{sech}^{2}(x)\right]^{\wedge}(p)=\pi p \operatorname{cosech}\left(\frac{\pi}{2} p\right),
$$

which is proved later in this chapter in section 2.4. The weight $\tanh \left(\frac{\pi}{2} p\right)$ introduced into the inner product means that the integral in (2.1.20) exists. Consequently, I can define the Fourier transform of $\tanh (x)$ in a distributional sense as

$$
[\tanh (x)]^{\wedge}(p)=-i \pi \operatorname{cosech}\left(\frac{\pi}{2} p\right) .
$$

I then obtain, in the same sense, the Fourier transform of $g_{1}(x)$, namely

$$
\widehat{g_{1}}(p)=G^{\prime}(\infty) \delta(p)-\pi i \operatorname{cosech}\left(\frac{\pi}{2} p\right) \widehat{h}(p) .
$$

From equations (2.1.24) and (2.1.14) respectively, I obtain

$$
\begin{aligned}
\widehat{h}(p) & =\frac{i}{\pi} \sinh \left(\frac{\pi}{2} p\right)\left[\widehat{g_{1}}(p)-G^{\prime}(\infty) \delta(p)\right] \\
& =\frac{1}{\pi} \cosh \left(\frac{\pi}{2} p\right) \widehat{g_{2}}(p) .
\end{aligned}
$$

My assumption that $h(x) \in L^{2}(\mathbb{R})$ means that $\widehat{g_{2}}(p)$ must tend to zero faster than $\operatorname{sech}\left(\frac{\pi}{2} p\right)$, and $\left|\widehat{g_{1}}(p)\right|$ must tend to zero faster than $\operatorname{cosech}\left(\frac{\pi}{2} p\right)$, as $p \rightarrow \pm \infty$. 
The factors multiplying $\widehat{g_{1}}(p)$ and $\widehat{g_{2}}(p)$ in (2.1.25) and (2.1.26) have exponential growth (with index $\frac{\pi}{2}$ ) as $|p| \rightarrow \infty$. Any noise present in $g_{1}$ and $g_{2}$ will have its Fourier transform amplified by a factor $e^{\frac{\pi}{2}|p|}$ as $|p| \rightarrow \infty$, which proves that the problem of determining the continuous relaxation spectrum from the storage and loss moduli is exponentially ill-posed with index $\frac{\pi}{2}$.

Since $k_{1}(x) \star 1=0$, I may also formally write the solutions of $(2.1 .10)$ and (2.1.11) as convolutions in the form

$$
\begin{aligned}
h(x) & =k_{1}(x) \star g_{1}(x) \\
& =k_{2}(x) \star g_{2}(x),
\end{aligned}
$$

where the distributions

$$
\begin{aligned}
& k_{1}(x)=\frac{i}{2 \pi^{2}} \int_{-\infty}^{\infty} \sinh \left(\frac{\pi}{2} p\right) e^{i p x} d p \\
& k_{2}(x)=\frac{1}{2 \pi^{2}} \int_{-\infty}^{\infty} \cosh \left(\frac{\pi}{2} p\right) e^{i p x} d p
\end{aligned}
$$

are defined through the inner products

$$
\begin{array}{ll}
\left\langle k_{1}(x), \phi(x)\right\rangle=\frac{i}{2 \pi^{2}} \int_{-\infty}^{\infty} \int_{-\infty}^{\infty} \sinh \left(\frac{\pi}{2} p\right) e^{i p x} \phi(x) d p d x & \forall \phi \in S_{\frac{\pi}{2}} \vee \\
\left\langle k_{2}(x), \phi(x)\right\rangle=\frac{1}{2 \pi^{2}} \int_{-\infty}^{\infty} \int_{-\infty}^{\infty} \cosh \left(\frac{\pi}{2} p\right) e^{i p x} \phi(x) d p d x & \forall \phi \in S_{\frac{\pi}{2}}^{\vee},
\end{array}
$$

where $S_{\frac{\pi}{2}}{ }^{\vee}$ is the set of all test functions whose Fourier transform lie in the space $S_{\frac{\pi}{2}}$. An example of such functions is the sequence of Gaussian functions of the form

$$
\phi_{m}(x)=\frac{1}{2 m^{3} \sqrt{\pi}} e^{-\frac{1}{2}\left(\frac{p}{m}\right)^{2}}, \quad m=1,2, \ldots,
$$

whose Fourier transform

$$
\widehat{\phi_{m}}(p)=\frac{1}{m} e^{-\frac{(m p)^{2}}{2}}, \quad m=1,2, \ldots
$$


is an element of $S_{\frac{\pi}{2}}$, from (2.1.17). A further example is the sequence of sech functions

$$
\phi_{m}(x)=\frac{\pi}{2 \lambda m^{2}} \operatorname{sech}\left(\frac{\pi}{2 m \lambda} x\right) \quad \lambda>\frac{\pi}{2}, \quad m=1,2, \ldots,
$$

whose Fourier transform

$$
\widehat{\phi_{m}}(p)=\frac{1}{m} \operatorname{sech}(m \lambda p) \quad \lambda>\frac{\pi}{2}, \quad m=1,2, \ldots,
$$

is an element of $S_{\frac{\pi}{2}}$, from (2.1.18).

\subsection{Double integral inversion formulae}

The formulations (2.1.27) and (2.1.28) were previously used by Davies and Anderssen (1997) [28] and Loy, Davies and Anderssen (2009) [48] in presenting the concept of sampling localization. Equations (2.1.27) and (2.1.28) also enable me to write double integral inversion formulae for $H(\tau)$ in terms of $G^{\prime}(\omega)$ and $G^{\prime \prime}(\omega)$. Returning to the physical variables $\omega$ and $\tau$, we have

$$
\begin{aligned}
H(\tau) & =\frac{i}{\pi^{2}} \int_{-\infty}^{\infty} \int_{-\infty}^{\infty} G^{\prime}(\omega) \sinh \left(\frac{\pi}{2} p\right) e^{i p \ln (\omega \tau)} d p d \ln \omega \\
& =\frac{1}{\pi^{2}} \int_{-\infty}^{\infty} \int_{-\infty}^{\infty} G^{\prime \prime}(\omega) \cosh \left(\frac{\pi}{2} p\right) e^{i p \ln (\omega \tau)} d p d \ln \omega
\end{aligned}
$$

The first of these two equations was discovered by Fuoss and Kirkwood (1941) [36]. The second was derived by Hussein (1997) [45] in a PhD thesis. It turns out that these two equations are two examples of several possible double integral inversion formulae relating functions of the form $\tau^{n} H(\tau)$ to data involving $\omega^{m} G^{*}(\omega)$. In this section I will develop a more expansive list, where I will show that there exist two separate double integral inversion formulae for $\tau^{n} H(\tau)$, for each value of $n \in \mathbb{Z}$.

For $n \in \mathbb{Z}, \mathrm{I}$ define the moments

$$
M_{n}=\int_{0}^{\infty} \tau^{n+1} H(\tau) \frac{d \tau}{\tau} .
$$


In particular, for $n=0$ and $n=-1$, I obtain the physical quantities

$$
\begin{aligned}
M_{0} & =\eta_{0}=\int_{0}^{\infty} H(\tau) d \tau, \\
M_{-1} & =G^{\prime}(\infty)=\int_{0}^{\infty} H(\tau) \frac{d \tau}{\tau} .
\end{aligned}
$$

The moments $M_{n}$ exist for a particular $n \in \mathbb{Z}$ if $H(\tau)$ has the following properties:

$$
\begin{aligned}
& \text { (1) } \quad H(\tau) \sim \tau^{\alpha-n} \quad \text { as } \quad \tau \rightarrow 0 \quad(\alpha>-1) \text {, } \\
& \text { (2) } \quad H(\tau) \sim \tau^{\beta-n} \text { as } \tau \rightarrow \infty \quad(\beta<-1) \text {. }
\end{aligned}
$$

Using the substitution

$$
x=\omega \tau,
$$

I can express the storage and loss moduli in the following form

$$
\begin{aligned}
G^{\prime}(\omega) & =\int_{0}^{\infty} \frac{x^{2}}{1+x^{2}} H(\tau) \frac{d \tau}{\tau} \\
G^{\prime \prime}(\omega) & =\int_{0}^{\infty} \frac{x}{1+x^{2}} H(\tau) \frac{d \tau}{\tau} .
\end{aligned}
$$

Starting with the relationships

$$
\begin{aligned}
\frac{\tau^{2} x^{2}}{1+x^{2}} & =\tau^{2}-\frac{\omega^{-2} x^{2}}{1+x^{2}} \\
\frac{\tau^{2} x}{1+x^{2}} & =\omega^{-1} \tau-\frac{\omega^{-2} x}{1+x^{2}} \\
\frac{\tau^{2 n} x^{2}}{1+x^{2}} & =\omega \frac{\tau^{2 n+1} x}{1+x^{2}}
\end{aligned}
$$

it can be proved by induction that

$$
\begin{aligned}
\int_{0}^{\infty} \frac{x^{2}}{1+x^{2}}\left[\tau^{2 n} H(\tau)\right] \frac{d \tau}{\tau}= & M_{2 n-1}-\omega^{-2} M_{2 n-3}+\ldots+(-1)^{n-1} \omega^{-2(n-1)} M_{1} \\
& +(-1)^{n} \omega^{-2 n} G^{\prime}(\omega) \quad \forall n \geq 0, \\
\int_{0}^{\infty} \frac{x}{1+x^{2}}\left[\tau^{2 n} H(\tau)\right] \frac{d \tau}{\tau}= & \omega^{-1} M_{2 n-2}-\omega^{-3} M_{2 n-4}+\ldots+(-1)^{n-1} \omega^{-2 n+1} M_{0} \\
& +(-1)^{n} \omega^{-2 n-1} G^{\prime \prime}(\omega) \quad \forall n \geq 0 .
\end{aligned}
$$


From (2.2.13), it follows that

$$
\begin{aligned}
& \int_{0}^{\infty} \frac{x^{2}}{1+x^{2}}\left[\tau^{2 n-1} H(\tau)\right] \frac{d \tau}{\tau}=\omega \int_{0}^{\infty} \frac{x}{1+x^{2}}\left[\tau^{2 n} H(\tau)\right] \frac{d \tau}{\tau} \quad \forall n \in \mathbb{Z} \\
& \int_{0}^{\infty} \frac{x}{1+x^{2}}\left[\tau^{2 n+1} H(\tau)\right] \frac{d \tau}{\tau}=\omega^{-1} \int_{0}^{\infty} \frac{x^{2}}{1+x^{2}}\left[\tau^{2 n} H(\tau)\right] \frac{d \tau}{\tau} \quad \forall n \in \mathbb{Z}
\end{aligned}
$$

I can similarly use the relationships

$$
\begin{aligned}
& \frac{\tau^{-1} x^{2}}{1+x^{2}}=\tau-\frac{\omega x}{1+x^{2}} \\
& \frac{\tau^{-2} x^{2}}{1+x^{2}}=\omega^{2}-\frac{\omega^{2} x^{2}}{1+x^{2}}
\end{aligned}
$$

to prove by induction that $\forall n \geq 0$,

$$
\begin{aligned}
\int_{0}^{\infty} \frac{x^{2}}{1+x^{2}}\left[\tau^{-(2 n+1)} H(\tau)\right] \frac{d \tau}{\tau}= & \omega^{2} M_{-2 n}-\omega^{4} M_{-2 n+2}+\ldots+(-1)^{n-1} \omega^{2 n} M_{-2} \\
& +(-1)^{n} \omega^{2 n+1} G^{\prime \prime}(\omega), \\
\int_{0}^{\infty} \frac{x^{2}}{1+x^{2}}\left[\tau^{-2 n} H(\tau)\right] \frac{d \tau}{\tau}= & \omega^{2} M_{-2 n+1}-\omega^{4} M_{-2 n+3}+\ldots+(-1)^{n-1} \omega^{2 n} M_{-1} \\
& +(-1)^{n} \omega^{2 n} G^{\prime}(\omega) .
\end{aligned}
$$

Formulae for $\int_{0}^{\infty} \frac{x}{1+x^{2}}\left[\tau^{-(2 n+1)} H(\tau)\right] \frac{d \tau}{\tau}$ and $\int_{0}^{\infty} \frac{x}{1+x^{2}}\left[\tau^{-2 n} H(\tau)\right] \frac{d \tau}{\tau}$ can be derived from equations (2.2.16) and (2.2.17). Double integral inversion formulae exist for each of (2.2.14)-(2.2.17) and (2.2.21)-(2.2.22). The following list contains the first twelve of these formulae. 


$$
\begin{aligned}
& \tau^{3} H(\tau)=\frac{1}{\pi^{2}} \int_{-\infty}^{\infty} \int_{-\infty}^{\infty} \omega^{-1}\left[M_{1}-\omega^{-2} G^{\prime}(\omega)\right] \cosh \left(\frac{\pi}{2} p\right) e^{i p \ln (\omega \tau)} d p d \ln \omega \\
& \tau^{2} H(\tau)=\frac{i}{\pi^{2}} \int_{-\infty}^{\infty} \int_{-\infty}^{\infty}\left[M_{1}-\omega^{-2} G^{\prime}(\omega)\right] \sinh \left(\frac{\pi}{2} p\right) e^{i p \ln (\omega \tau)} d p d \ln \omega \\
& =\frac{1}{\pi^{2}} \int_{-\infty}^{\infty} \int_{-\infty}^{\infty} \omega^{-1}\left[\eta_{0}-\omega^{-1} G^{\prime \prime}(\omega)\right] \cosh \left(\frac{\pi}{2} p\right) e^{i p \ln (\omega \tau)} d p d \ln \omega \\
& \tau H(\tau)=\frac{1}{\pi^{2}} \int_{-\infty}^{\infty} \int_{-\infty}^{\infty} \omega^{-1} G^{\prime}(\omega) \cosh \left(\frac{\pi}{2} p\right) e^{i p \ln (\omega \tau)} d p d \ln \omega \\
& =\frac{i}{\pi^{2}} \int_{-\infty}^{\infty} \int_{-\infty}^{\infty}\left[\eta_{0}-\omega^{-1} G^{\prime \prime}(\omega)\right] \sinh \left(\frac{\pi}{2} p\right) e^{i p \ln (\omega \tau)} d p d \ln \omega, \\
& H(\tau)=\frac{i}{\pi^{2}} \int_{-\infty}^{\infty} \int_{-\infty}^{\infty} G^{\prime}(\omega) \sinh \left(\frac{\pi}{2} p\right) e^{i p \ln (\omega \tau)} d p d \ln \omega \\
& =\frac{1}{\pi^{2}} \int_{-\infty}^{\infty} \int_{-\infty}^{\infty} G^{\prime \prime}(\omega) \cosh \left(\frac{\pi}{2} p\right) e^{i p \ln (\omega \tau)} d p d \ln \omega, \\
& \tau^{-1} H(\tau)=\frac{1}{\pi^{2}} \int_{-\infty}^{\infty} \int_{-\infty}^{\infty} \omega\left[G^{\prime}(\infty)-G^{\prime}(\omega)\right] \cosh \left(\frac{\pi}{2} p\right) e^{i p \ln (\omega \tau)} d p d \ln \omega \\
& =\frac{i}{\pi^{2}} \int_{-\infty}^{\infty} \int_{-\infty}^{\infty} \omega G^{\prime \prime}(\omega) \sinh \left(\frac{\pi}{2} p\right) e^{i p \ln (\omega \tau)} d p d \ln \omega \\
& \tau^{-2} H(\tau)=\frac{i}{\pi^{2}} \int_{-\infty}^{\infty} \int_{-\infty}^{\infty} \omega^{2}\left[G^{\prime}(\infty)-G^{\prime}(\omega)\right] \sinh \left(\frac{\pi}{2} p\right) e^{i p \ln (\omega \tau)} d p d \ln \omega \\
& =\frac{1}{\pi^{2}} \int_{-\infty}^{\infty} \int_{-\infty}^{\infty} \omega\left[M_{-2}-\omega G^{\prime \prime}(\omega)\right] \cosh \left(\frac{\pi}{2} p\right) e^{i p \ln (\omega \tau)} d p d \ln \omega \\
& \tau^{-3} H(\tau)=\frac{i}{\pi^{2}} \int_{-\infty}^{\infty} \int_{-\infty}^{\infty} \omega^{2}\left[M_{-2}-\omega G^{\prime \prime}(\omega)\right] \sinh \left(\frac{\pi}{2} p\right) e^{i p \ln (\omega \tau)} d p d \ln \omega
\end{aligned}
$$




\subsubsection{Existence of Moments}

From equation (1.2.5) (for viscoelastic liquids), the relaxation function $G(t)$ can be expressed as

$$
G(t)=\int_{0}^{\infty} H(\tau) e^{-\frac{t}{\tau}} \frac{d \tau}{\tau}
$$

Comparing the above equation with my definition of the $n$-th moment of $H(\tau)(2.2 .3)$, I derive that

$$
\begin{aligned}
G^{n}(0) & =(-1)^{n} \int_{0}^{\infty} \tau^{-n} H(\tau) \frac{d \tau}{\tau} \\
& =(-1)^{n} M_{-(n+1)} .
\end{aligned}
$$

Furthermore, by using equation (1.3.4), with $G_{e}=0$, the complex shear modulus $G^{*}(\omega)$ can be expressed as the half Fourier transform of the relaxation function, i.e.

$$
G^{*}(\omega)=i \omega \int_{0}^{\infty} G(t) e^{-i \omega t} d t
$$

Also, the Laplace transform of $G(t)$ is defined as

$$
\mathcal{L}\{G(t)\}=\int_{0}^{\infty} G(t) e^{-s t} d t
$$

If $G(t)$ has a Maclaurin series, then the Laplace transform of $G(t)$ has an asymptotic expansion (as $s \rightarrow \infty$ ) of the form

$$
\mathcal{L}\{G(t)\} \sim \frac{\gamma_{0}}{s}+\frac{\gamma_{1}}{s^{2}}+\frac{\gamma_{2}}{s^{3}}+\ldots
$$

where

$$
\gamma_{n}=\frac{1}{n !} G^{(n)}(0)=\frac{(-1)^{n}}{n !} M_{-(n+1)} .
$$

Consequently, a sufficient condition for all negative moments of $H(\tau)$ to exist is that the relaxation function $G(t)$ has a Maclaurin series. However, 
in general, not all continuous relaxation spectra lead to a relaxation function with a Maclaurin series. I now give an example of a relaxation function that does not have a Maclaurin series, yet I can adjust a scaling parameter $\sigma$ to get as many moments of $H(\tau)$ as I like.

Theorem 2.1. If

$$
H(\tau)=\operatorname{sech}\left(\frac{\ln \tau}{\sigma}\right)
$$

then for $\frac{1}{b+1}<\sigma<\frac{1}{b}\left(b \in \mathbb{Z}^{+}\right)$, there will exist $2 b+1$ moments of $H(\tau)$.

The reason for the above choice of hyperbolic wavelets in the representation of $H(\tau)$ will become apparent over the next few chapters. In Chapter 5, I will use a sum of translated sech functions, scaled by $\sigma$, in which I will show that for a particular set of real data (known as PB1 data), the optimal value of $\sigma$ is in the range $\frac{1}{3}<\sigma<\frac{1}{2}$. Using the above theorem, this means that the five moments $M_{-3}, M_{-2}, M_{-1}, M_{0}$ and $M_{1}$ exist. For this range of values for $\sigma$, all twelve of the double integral inversion formulae in (2.2.23)-(2.2.34) become relevant.

Using equations (2.2.35) and (2.2.41), the relaxation function can be written as

$$
\begin{aligned}
G(t) & =\int_{0}^{\infty} \operatorname{sech}\left(\frac{\ln \tau}{\sigma}\right) e^{-\frac{t}{\tau}} \frac{d \tau}{\tau} \\
& =\int_{0}^{\infty} \frac{1}{s} \operatorname{sech}\left(\ln \left[s^{\frac{1}{\sigma}}\right]\right) e^{-s t} d s \quad\left(s=\frac{1}{\tau}\right) \\
& =\int_{0}^{\infty} \frac{2 s^{\frac{1}{\sigma}-1}}{1+s^{\frac{2}{\sigma}}} e^{-s t} d s .
\end{aligned}
$$

Since $\gamma_{n} \propto M_{-(n+1)}$, if not all of the negative moments of $H(\tau)$ exist, then $G(t)$ does not have a Maclaurin series. Indeed, the moments of $H(\tau)$ are of 
the form

$$
\begin{aligned}
M_{n} & =\int_{0}^{\infty} \tau^{n+1} \operatorname{sech}\left(\frac{\ln \tau}{\sigma}\right) \frac{d \tau}{\tau} \\
& =\int_{-\infty}^{\infty} e^{-(n+1) t} \operatorname{sech}\left(\frac{t}{\sigma}\right) d t \quad(t=-\ln \tau) \\
& =2 \int_{-\infty}^{\infty} \frac{e^{-\left(n+\frac{1}{\sigma}+1\right) t}}{1+e^{-\frac{2 t}{\sigma}}} d t
\end{aligned}
$$

Let $b<\frac{1}{\sigma}<b+1$ (i.e. $\frac{1}{b+1}<\sigma<\frac{1}{b}$ ). Then, for $M_{n}$ to exist, we require $-(b+1) \leq n \leq b-1$. This gives us $2 b+1$ moments of $H(\tau)$, namely $M_{-b-1}, M_{-b}, \ldots, M_{0}, \ldots, M_{b-2}, M_{b-1}$. Since $\gamma_{n} \propto M_{-(n+1)}$, only $b+1$ of the $\gamma_{n}$ exist, namely $\gamma_{0}, \gamma_{1}, \ldots, \gamma_{b}$. I have now shown an example of a well behaved relaxation function that does not have a Maclaurin series, since only finitely many of the $\gamma_{n}$ exist.

\subsection{The Fourier transform of $\operatorname{sech}(x)$}

To obtain the Fourier transform of $\operatorname{sech}(x)$, I use the same method as shown by Priestley [64], where the integral $\int_{-\infty}^{\infty} \operatorname{sech}(x) e^{a x} d x$ was determined. I have exchanged $-i p$ for $a$.

$$
\begin{aligned}
\widehat{\operatorname{sech}}(p) & =\int_{-\infty}^{\infty} \operatorname{sech}(x) e^{-i p x} d x \\
& =\int_{-\infty}^{\infty} \frac{e^{-i p x}}{\cosh (x)} d x
\end{aligned}
$$

Let

$$
f(z)=\frac{g(z)}{h(z)}
$$

where

$$
g(z)=e^{-i p z}
$$


and

$$
h(z)=\cosh (z) .
$$

The function $f(z)$ has simple poles at $z=\frac{1}{2}(2 n+1) \pi i, n \in \mathbb{N}$. The function $f$ is holomorphic inside and on the contour $\gamma$ shown in the Fig. 2.1 below, except for a simple pole inside $\gamma$ at $z=\frac{\pi}{2} i$, of residue

$$
\begin{aligned}
\frac{g\left(\frac{\pi}{2} i\right)}{h^{\prime}\left(\frac{\pi}{2} i\right)} & =\frac{e^{\frac{\pi}{2} p}}{\sinh \left(\frac{\pi}{2} i\right)} \\
& =-i e^{\frac{\pi}{2} p}
\end{aligned}
$$

By Cauchy's residue theorem [64],



Figure 2.1: Contour $\gamma$.

$$
\begin{aligned}
& \int_{-S}^{R} \frac{e^{-i p x}}{\cosh (x)} d x+\int_{0}^{\pi} \frac{i e^{-i p(R+i y)}}{\cosh (R+i y)} d y+\int_{R}^{-S} \frac{e^{\pi p} e^{-i p x}}{\cosh (x+\pi i)} d x+\int_{\pi}^{0} \frac{i e^{-i p(-S+i y)}}{\cosh (-S+i y)} d y \\
= & 2 \pi i \operatorname{Res}\left(f, \frac{\pi}{2} i\right) \\
= & 2 \pi e^{\frac{\pi}{2} p} .
\end{aligned}
$$

Denote the second integral by $I$ and the fourth by $J$. Then

$$
\begin{aligned}
|I| & \leq \int_{0}^{\pi}\left|\frac{i e^{-i p(R+i y)}}{\cosh (R+i y)}\right| d y \\
& \leq \int_{0}^{\pi} \frac{2 e^{p y}}{\left|e^{R+i y}+e^{-(R+i y)}\right|} d y .
\end{aligned}
$$


So $I \rightarrow 0$ as $R \rightarrow \infty$. Similarly,

$$
|J| \leq \int_{0}^{\pi} \frac{2 e^{p y}}{\mid e^{-S+i y}+e^{S-i y \mid}} d y .
$$

So $J \rightarrow 0$ as $S \rightarrow \infty$. Letting $R$ and $S$ tend to $\infty$ independently, we obtain

$$
\begin{aligned}
2 \pi e^{\frac{\pi}{2} p} & =\int_{-\infty}^{\infty} \frac{e^{-i p x}}{\cosh (x)} d x+\int_{\infty}^{-\infty} \frac{e^{\pi p} e^{-i p x}}{\cosh (x+\pi i)} d x \\
& =\int_{-\infty}^{\infty} e^{-i p x}\left(\frac{1}{\cosh (x)}-\frac{e^{\pi p}}{\cosh (x+\pi i)}\right) d x \\
& =\int_{-\infty}^{\infty} \frac{e^{-i p x}}{\cosh (x)}\left(1+e^{\pi p}\right) d x
\end{aligned}
$$

since

$$
\cosh (x+\pi i)=-\cosh (x) .
$$

Consequently, the Fourier transform of $\operatorname{sech}(x)$ is given by

$$
\begin{aligned}
\int_{-\infty}^{\infty} \frac{e^{-i p x}}{\cosh (x)} d x & =\frac{2 \pi e^{\frac{\pi}{2} p}}{1+e^{\pi p}} \\
& =\frac{2 \pi}{e^{\frac{\pi}{2} p}+e^{-\frac{\pi}{2} p}} \\
& =\pi \operatorname{sech}\left(\frac{\pi}{2} p\right) .
\end{aligned}
$$

\subsection{The Fourier transform of $\operatorname{sech}^{2}(x)$}

To obtain the Fourier transform of $\operatorname{sech}^{2}(x)$, I use the same method (and the same contour) as I used to obtain the Fourier transform of $\operatorname{sech}(x)$ above.

$$
\begin{aligned}
\widehat{\operatorname{sech}^{2}}(p) & =\int_{-\infty}^{\infty} \operatorname{sech}^{2}(x) e^{-i p x} d x \\
& =\int_{-\infty}^{\infty} \frac{e^{-i p x}}{\cosh ^{2}(x)} d x
\end{aligned}
$$


Let

$$
f(z)=\frac{e^{-i p z}}{\cosh ^{2}(z)}
$$

The function $f(z)$ has double poles at $z=\frac{1}{2}(2 n+1) \pi i, n \in \mathbb{N}$. Since there exists a double pole at $z=\frac{\pi}{2} i$, I consider a Laurent expansion of $f(z)$ at $z=\frac{\pi}{2} i$. The Taylor expansion of $e^{-i p z}$ at $z=\frac{\pi}{2} i$ is

$$
e^{\frac{\pi}{2} p}\left[1-i p\left(z-\frac{\pi}{2} i\right)+\ldots\right]
$$

The Taylor expansion of $\cosh (z)$ at $z=\frac{\pi}{2} i$ is

$$
i\left(z-\frac{\pi}{2} i\right)\left[1+\frac{1}{6}\left(z-\frac{\pi}{2} i\right)^{2}+\ldots\right] .
$$

Hence, the Taylor expansion of $\cosh ^{2}(z)$ at $z=\frac{\pi}{2} i$ is

$$
-\left(z-\frac{\pi}{2} i\right)^{2}\left[1+\frac{1}{3}\left(z-\frac{\pi}{2} i\right)^{2}+\ldots\right]
$$

The Laurent expansion of $f(z)$ at $z=\frac{\pi}{2} i$ is therefore

$$
\begin{aligned}
& -\frac{e^{\frac{\pi}{2} p}\left[1-i p\left(z-\frac{\pi}{2} i\right)+\ldots\right]}{\left(z-\frac{\pi}{2} i\right)^{2}\left[1+\frac{1}{3}\left(z-\frac{\pi}{2} i\right)^{2}+\ldots\right]} \\
= & -\frac{e^{\frac{\pi}{2} p}\left[1-i p\left(z-\frac{\pi}{2} i\right)+\ldots\right]\left[1-\frac{1}{3}\left(z-\frac{\pi}{2} i\right)^{2}-\ldots\right]}{\left(z-\frac{\pi}{2} i\right)^{2}} .
\end{aligned}
$$

The residue of $f(z)$ at the double pole $z=\frac{\pi}{2} i$ is equal to the coefficient of $\left(z-\frac{\pi}{2} i\right)^{-1}$ in the Laurent expansion. Consequently,

$$
\operatorname{Res}\left(f, \frac{\pi}{2} i\right)=i p e^{\frac{\pi}{2} p}
$$


By Cauchy's residue theorem, as $R, S \rightarrow \infty$ independently, we find that

$$
\begin{aligned}
& \int_{-\infty}^{\infty} \frac{e^{-i p x}}{\cosh ^{2}(x)} d x-\int_{-\infty}^{\infty} \frac{e^{-i p(x+\pi i)}}{\cosh ^{2}(x+\pi i)} d x=2 \pi i \operatorname{Res}\left(f, \frac{\pi}{2} i\right) \\
\therefore \quad & \left(1-e^{\pi p}\right) \int_{-\infty}^{\infty} \frac{e^{-i p x}}{\cosh ^{2}(x)} d x=-2 \pi p e^{\frac{\pi}{2} p} \\
\therefore \quad & \int_{-\infty}^{\infty} \frac{e^{-i p x}}{\cosh ^{2}(x)} d x=\frac{2 \pi p}{e^{\frac{\pi}{2} p}-e^{-\frac{\pi}{2} p}} \\
\therefore \quad & \operatorname{sech}^{2}(p)=\pi p \operatorname{cosech}\left(\frac{\pi}{2} p\right) .
\end{aligned}
$$

\subsection{Conclusion}

I have shown in this chapter that recovery of the continuous relaxation spectrum is an exponentially ill-posed inverse problem. To enable me to do this, I have introduced aspects of the theory of distributions. I also required the Fourier transforms of $\operatorname{sech}(x)$ and $\operatorname{sech}^{2}(x)$, for which I have provided rigorous derivations.

I introduced generalized formulae for double integral inversion formulae relating moments of the relaxation spectrum to data involving $\omega^{m} G^{*}(\omega)$. I have also given a specific example of a well-behaved relaxation spectrum that does not have a Maclaurin series. 


\section{Chapter 3}

\section{Wavelets Transforms in the Theory of Linear Viscoelasticity}

The convolution equations (2.1.10) and (2.1.11) are intimately linked to continuous wavelet transforms, where the wavelets are constructed from simple hyperbolic functions. It will be shown that this link leads to a natural representation of the continuous spectrum as a wavelet series.

\subsection{Continuous wavelets}

The wavelets that will be encountered in this thesis are real valued functions, which satisfy strict admissibility and regularity criteria. They are localized in time. Wavelets replace the non-localized trigonometric functions in the Fourier transform to provide a much more powerful analytic tool.

Definition 3.1. I define a mother wavelet as a function $\psi \in L^{2}(\mathbf{R})$ which has zero average

$$
\int_{-\infty}^{\infty} \psi(x) d x=0
$$


and has the property that there exists a constant $C_{\psi}$ such that

$$
C_{\psi}=\int_{0}^{\infty} \frac{|\widehat{\psi}(p)|^{2}}{|p|} d p<\infty
$$

This property means that $\psi$ can be paired with a scaling function $\phi$. For each $\psi$ there exists a unique real valued function $\phi \in L^{2}(\mathbb{R})$ whose Fourier transform is defined by

$$
\widehat{\phi}(p)=\left[\int_{p}^{\infty} \frac{|\widehat{\psi}(\xi)|^{2}}{\xi} d \xi\right]^{\frac{1}{2}}
$$

$\phi$ is an even function which has non-zero area given by

$$
\widehat{\phi}(0)=\left(C_{\psi}\right)^{\frac{1}{2}}
$$

$\psi$ is often called a mother wavelet with corresponding scaling function $\phi . \phi$ is also called the corresponding father wavelet for $\psi$.

At different time locations $t$, and different time scales $s$ (resolution with respect to time) the mother and father wavelets generate a family of wavelets

$$
\psi_{s, t}(x)=\frac{1}{\sqrt{s}} \psi\left(\frac{x-t}{s}\right), \quad \phi_{s, t}(x)=\frac{1}{\sqrt{s}} \phi\left(\frac{x-t}{s}\right) .
$$

In terms of the original variables $\omega$ and $\tau$

$$
\psi_{s, t}(x)=\frac{1}{\sqrt{s}} \psi\left(\frac{\ln (\omega \tau)}{s}\right), \quad \phi_{s, t}(x)=\frac{1}{\sqrt{s}} \phi\left(\frac{\ln (\omega \tau)}{s}\right) .
$$

If the continuous spectrum is represented as a wavelet series, each term in the series will be centred at a relaxation time determined by a reciprocal frequency, i.e. $\tau=\omega^{-1}$, and the resolution obtainable from each term will depend on the scale $s$ associated with that term, small $s$ implying high resolution and large $s$ implying low resolution. 


\subsubsection{Continuous wavelet transforms}

The (continuous) wavelet transform of $h \in L^{2}(\mathbb{R})$ at the time $t$ and scale $s$ is defined by the convolution product

$$
\begin{aligned}
{[W h](s, t) } & =\left(h \star \psi_{s}\right)(t) \\
\text { where } \quad \psi_{s}(x) & =\psi_{s, 0}(x)=\frac{1}{\sqrt{s}} \psi\left(\frac{x}{s}\right)
\end{aligned}
$$

I define a father wavelet transform of $h \in L^{2}(\mathbb{R})$ by

$$
\begin{aligned}
{[V h](s, t) } & =\left(h \star \phi_{s}\right)(t) \\
\text { where } \quad \phi_{s}(x) & =\phi_{s, 0}(x)=\frac{1}{\sqrt{s}} \phi\left(\frac{x}{s}\right),
\end{aligned}
$$

and $\phi \in L^{2}(\mathbb{R})$ is the scaling function associated with a wavelet $\psi$.

The next statement is a fundamental observation which motivates the main results of this thesis. Equation (2.1.11) shows that, in terms of logfrequency $x$, the loss modulus is a father wavelet transform of the continuous relaxation spectrum, at unit scaling $s=1$, i.e.

$$
\begin{aligned}
g_{2}(x) & =(h \star \phi)(x)=[V h](1, x) \\
\text { where } \quad \phi(x) & =\operatorname{sech}(x) .
\end{aligned}
$$

In physical variables,

$$
G^{\prime \prime}(\omega)=\frac{1}{2}[V h](1, \ln \omega)
$$

\subsubsection{Autoconvoluted wavelets}

I now introduce autoconvoluted wavelets, because the inversion formulae that I am about to use to reconstruct the continuous relaxation spectrum, makes use of the autoconvoluted wavelets rather than the wavelets themselves. Throughout this thesis I will use the convenient property of the 
Fourier transform of a convolution, which gives

$$
(\psi \star \psi)^{\wedge}(p)=[\widehat{\psi}(p)]^{2}, \quad(\phi \star \phi)^{\wedge}(p)=[\widehat{\phi}(p)]^{2} .
$$

The autoconvolution of the mother wavelet is also a mother wavelet since it has zero area, shown by

$$
(\psi \star \psi)^{\wedge}(0)=[\widehat{\psi}(0)]^{2}=0
$$

and as long as it satisfies the condition

$$
\int_{0}^{\infty} \frac{\left|(\psi \star \psi)^{\wedge}(p)\right|^{2}}{|p|} d p=\int_{0}^{\infty} \frac{|\widehat{\psi}(p)|^{4}}{|p|} d p<\infty
$$

The autoconvolution of the father wavelet is also a father wavelet since it has constant area given by

$$
(\phi \star \phi)^{\wedge}(0)=[\widehat{\phi}(0)]^{2}=C_{\psi} .
$$

By manipulating equation (3.1.3), using properties of the Fourier transform given in Table 1.1, I derive the following relationship between the autoconvoluted mother wavelet and the autoconvoluted father wavelet

$$
\begin{aligned}
|\widehat{\phi}(p)|^{2} & =\int_{p}^{\infty} \frac{|\widehat{\psi}(\xi)|^{2}}{\xi} d \xi \\
\therefore[\phi \star \phi]^{\wedge}(p) & =\int_{p}^{\infty} \frac{[\psi \star \psi]^{\wedge}(\xi)}{\xi} d \xi \\
\therefore \frac{d}{d p}[\phi \star \phi]^{\wedge}(p) & =-\frac{[\psi \star \psi]^{\wedge}(p)}{p} \\
\therefore[\psi \star \psi]^{\wedge}(p) & =-p \frac{d}{d p}[\phi \star \phi]^{\wedge}(p) \\
& =i p[x(\phi \star \phi)(x)]^{\wedge}(p) \\
& =\left[\frac{d}{d x}(x(\phi \star \phi)(x))\right]^{\wedge}(p) \\
\therefore(\psi \star \psi)(x) & =\frac{d}{d x}(x(\phi \star \phi)(x)) \\
& =(\phi \star \phi)(x)+x \frac{d}{d x}(\phi \star \phi)(x) .
\end{aligned}
$$


This proves that the autoconvoluted mother wavelet is equal to the autoconvoluted father wavelet plus its homogeneous derivative.

\subsection{Inversion Formulae}

Calderón in 1964 laid the ground for a theory of continuous wavelet transforms and their inversion by publishing his famous "decomposition of the identity".

Theorem 3.1 [19]. Every function $h \in L^{2}(\mathbb{R})$ can be decomposed into a sum over all scales of its wavelet transforms

$$
\begin{aligned}
h(t) & =\frac{1}{C_{\psi}} \int_{0}^{\infty}\left(h \star \bar{\psi}_{s} \star \psi_{s}\right)(t) \frac{d s}{s^{2}} \\
\text { where } \quad \bar{\psi}_{s}(t) & =\frac{1}{\sqrt{s}} \psi\left(\frac{-t}{s}\right) .
\end{aligned}
$$

Remark: The integral in (3.2.1) converges in the $L^{2}$-norm to $h(t)$. The integral with respect to $t$ of the function on the right of (3.2.1) vanishes, while the integral of $h(t)$ on the left does not vanish. So the right-hand side of (3.2.1) does not converge pointwise to $h(t)$. We shall see how to overcome this difficulty below using scaling functions.

In this thesis, unless stated otherwise, I will use even functions $\psi(t)$. Therefore

$$
\bar{\psi}_{s}(t)=\psi_{s}(t)
$$

and I may consequently re-write equation (3.2.1) as

$$
h(t)=\frac{1}{C_{\psi}} \int_{0}^{\infty}\left(h \star \psi_{s} \star \psi_{s}\right)(t) \frac{d s}{s^{2}} .
$$


I can assert that this is a valid decomposition of the identity as follows

$$
\begin{aligned}
h(t) & =\frac{1}{C_{\psi}} \int_{0}^{\infty}\left(h \star \psi_{s} \star \psi_{s}\right)(t) \frac{d s}{s^{2}} \\
& =\frac{1}{C_{\psi}} h(t) \star \int_{0}^{\infty}\left(\psi_{s} \star \psi_{s}\right)(t) \frac{d s}{s^{2}} \\
& =\frac{1}{C_{\psi}} h(t) \star\left\{\left[\int_{0}^{\infty}\left(\psi_{s} \star \psi_{s}\right)(t) \frac{d s}{s^{2}}\right]^{\wedge}(p)\right\}^{\vee}(t) \\
& =\frac{1}{C_{\psi}} h(t) \star\left\{\int_{0}^{\infty}\left[\left(\psi_{s} \star \psi_{s}\right)(t)\right]^{\wedge}(p) \frac{d s}{s^{2}}\right\}^{\vee}(t) \\
& =\frac{1}{C_{\psi}} h(t) \star\left\{\int_{0}^{\infty}\left[\widehat{\psi}_{s}(p)\right]^{2} \frac{d s}{s^{2}}\right\}^{\vee}(t) \\
& =\frac{1}{C_{\psi}} h(t) \star\left\{\int_{0}^{\infty}[\widehat{\psi}(s p)]^{2} \frac{d s}{s}\right\}^{\vee}(t),
\end{aligned}
$$

since

$$
\psi_{s}(x)=\frac{1}{\sqrt{s}} \psi\left(\frac{x}{s}\right) \Rightarrow \widehat{\psi}_{s}(p)=\sqrt{s} \widehat{\psi}(s p)
$$

and consequently

$$
\left[\widehat{\psi}_{s}(p)\right]^{2}=s[\widehat{\psi}(s p)]^{2}
$$

Using the transformation $\xi=s p, p \neq 0, \mathrm{I}$ obtain

$$
\begin{aligned}
h(t) & =\frac{1}{C_{\psi}} h(t) \star\left\{\int_{0}^{\infty} \frac{[\widehat{\psi}(\xi)]^{2}}{\xi} d \xi\right\}(t) \\
& =\frac{1}{C_{\psi}} h(t) \star\left\{C_{\psi}\right\}^{\vee}(t) \quad(\text { using }(3.1 .2)) \\
& =(h \star \delta)(t), \quad \text { since } \hat{\delta}(p)=1 .
\end{aligned}
$$

Given that the wavelet transforms $h \star \psi_{s}$ of $h$ at all scales $s>0,(3.2 .4)$ gives an inversion formula for reconstructing $h(t)$ from its wavelet transforms. 
Since the areas of the graphs $h \star \psi_{s}$ and $h \star \psi_{s} \star \psi_{s}$ vanish for all $s>0$, then the restriction of (3.2.4) to any finite scale $\sigma$

$$
h_{\sigma}(t)=\frac{1}{C_{\psi}} \int_{0}^{\sigma}\left(h \star \psi_{s} \star \psi_{s}\right)(t) \frac{d s}{s^{2}}
$$

has a graph of zero area, i.e.

$$
\int_{-\infty}^{\infty} h_{\sigma}(t) d t=0, \text { for all } 0<\sigma<\infty
$$

This means that although the integral in (3.2.4) converges to $h(t)$ in the $L^{2}$-norm, it will not in general reproduce the area under the graph of $h(t)$, i.e.

$$
\lim _{\sigma \rightarrow \infty} \int_{-\infty}^{\infty} h_{\sigma}(t) d t \neq \int_{-\infty}^{\infty} h(t) d t
$$

In 1989, Mallat showed how to overcome this problem by splitting the integral into two parts [53]

$$
\begin{aligned}
h(t)= & \frac{1}{C_{\psi}} \int_{0}^{\sigma}\left(h \star \psi_{s} \star \psi_{s}\right)(t) \frac{d s}{s^{2}}+\frac{1}{C_{\psi} \sigma}\left(h \star \phi_{\sigma} \star \phi_{\sigma}\right)(t) \\
& \text { where } \phi_{s}(t)=\frac{1}{\sqrt{ } s} \phi\left(\frac{t}{s}\right) .
\end{aligned}
$$

The sum over all scales $s>\sigma$ can be incorporated into one term, by means of the scaling function $\phi_{\sigma}$. This term has the same area as $h(t)$, since $\left[\widehat{\phi_{\sigma}}(0)\right]^{2}=$ $C_{\psi} \sigma$. Furthermore, if $\sigma$ is sufficiently small, high resolution terms (small scales) are confined to the integral term in (3.2.10), while the second term constitutes a low-pass filter applied to $h$.

I can confirm that (3.2.10) is a valid decomposition of the identity by first 
using equation (3.2.5), with $0 \leq s \leq \sigma$

$$
\begin{aligned}
h(t) & =\frac{1}{C_{\psi}} \int_{0}^{\sigma}\left(h \star \psi_{s} \star \psi_{s}\right)(t) \frac{d s}{s^{2}}+\frac{1}{C_{\psi} \sigma}\left(h \star \phi_{\sigma} \star \phi_{\sigma}\right)(t) \\
& =\frac{1}{C_{\psi}} h(t) \star\left\{\int_{0}^{\sigma}[\widehat{\psi}(s p)]^{2} \frac{d s}{s}\right\}^{\vee}(t)+\frac{1}{C_{\psi} \sigma}\left(h \star \phi_{\sigma} \star \phi_{\sigma}\right)(t)
\end{aligned}
$$

Using properties of the Fourier transform (see Table 1.1), I can manipulate equation (3.1.3) to obtain

$$
\begin{aligned}
\widehat{\psi}^{2}(p) & =-p \frac{d}{d p} \widehat{\phi}^{2}(p) \\
\therefore \widehat{\psi}^{2}(s p) & =-p \frac{d}{d p} \widehat{\phi}^{2}(s p)
\end{aligned}
$$

Therefore, using (3.2.13) and the transformation $\xi=p s,(3.2 .12)$ becomes

$$
\begin{aligned}
h(t) & =-\frac{1}{C_{\psi}} h(t) \star\left\{\int_{0}^{\sigma p} \frac{d}{d \xi} \widehat{\phi}^{2}(\xi) d \xi\right\}^{\vee}(t)+\frac{1}{C_{\psi} \sigma}\left(h \star \phi_{\sigma} \star \phi_{\sigma}\right)(t) \\
& =\frac{1}{C_{\psi}} h(t) \star\{\widehat{\phi \star \phi}(0)-\widehat{\phi \star \phi}(\sigma p)\}^{\vee}(t)+\frac{1}{C_{\psi} \sigma}\left(h \star \phi_{\sigma} \star \phi_{\sigma}\right)(t) \\
& =\frac{1}{C_{\psi}} h(t) \star\left\{C_{\psi}-\widehat{\phi \star \phi}(\sigma p)\right\}^{\vee}(t)+\frac{1}{C_{\psi} \sigma}\left(h \star \phi_{\sigma} \star \phi_{\sigma}\right)(t) \\
& =(h \star \delta)(t)-\frac{1}{C_{\psi} \sigma}\left(h \star \phi_{\sigma} \star \phi_{\sigma}\right)(t)+\frac{1}{C_{\psi} \sigma}\left(h \star \phi_{\sigma} \star \phi_{\sigma}\right)(t) . \\
& =(h \star \delta)(t) .
\end{aligned}
$$

\subsection{An illustrative example}

To illustrate what the effect of changing $\sigma$ is in the Calderón-Mallat decomposition, I shall give a numerical example in which $h(t)$ is chosen to be a Gaussian. Let

$$
\begin{aligned}
h(t) & =e^{-\frac{t^{2}}{2}} \\
\left(\phi_{\sigma} \star \phi_{\sigma}\right)(t) & =\frac{1}{\sigma} \operatorname{sech}\left(\frac{t}{\sigma}\right) .
\end{aligned}
$$


I have plotted the Gaussian against its Calderón and Mallat parts for various values of $\sigma$ in Figs. 3.1 and 3.2. The Gaussian is plotted in black, the Calderón part in pink and the Mallat part in blue. We see that (Fig. 3.1) the Mallat term always captures the area under $h$ but if $\sigma>\frac{1}{8}$ then both terms are needed to give an accurate representation of $h$. The Calderón term is small when $\sigma<\frac{1}{8}$. When $\sigma=\frac{1}{16}$ (Fig. 3.2) the Mallat term alone represents $h$ with a maximum error of $5 \times 10^{-3}$. This clearly illustrates the fact that if $h(t)$ is a smooth function then high accuracy can be obtained even though high resolution terms (i.e. small scales) are excluded.

\subsection{FK approximation}

Ferry [33] attributes the simplest approximation to the continuous relaxation spectrum to Fuoss and Kirkwood [36]. This approximation takes the form

$$
H(\tau) \approx \frac{2}{\pi} G^{\prime \prime}\left(\tau^{-1}\right)
$$

It was derived by Fuoss and Kirkwood in the context of dielectric media, but is equally relevant in the case of linear viscoelasticity. I shall call approximation (3.4.1) the FK approximation.

Equation (3.2.10) enables me to derive, in integral form, an expression for the error in the FK approximation. I believe that no such error term has so far been available. Let $\sigma=1$, and choose

$$
(\phi \star \phi)(t)=\operatorname{sech}(t) .
$$

It then follows from (3.1.17), (3.1.16) and (2.1.13) that

$$
(\psi \star \psi)(t)=\operatorname{sech}(t)[1-t \tanh (t)]
$$

and

$$
C_{\psi}=\pi
$$




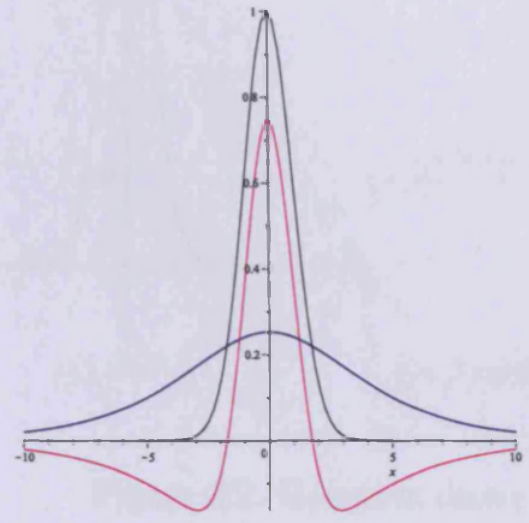

(a) $\sigma=3$

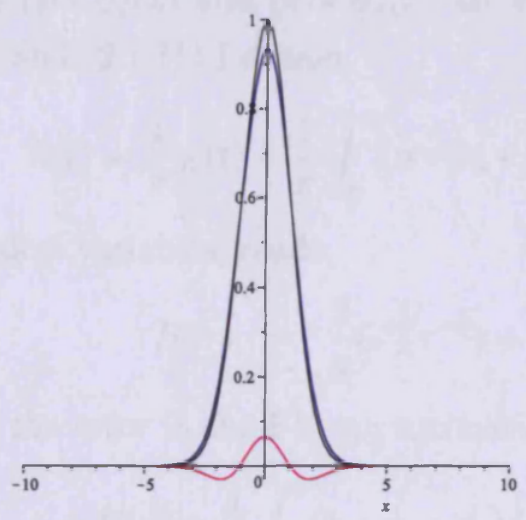

(c) $\sigma=\frac{1}{4}$

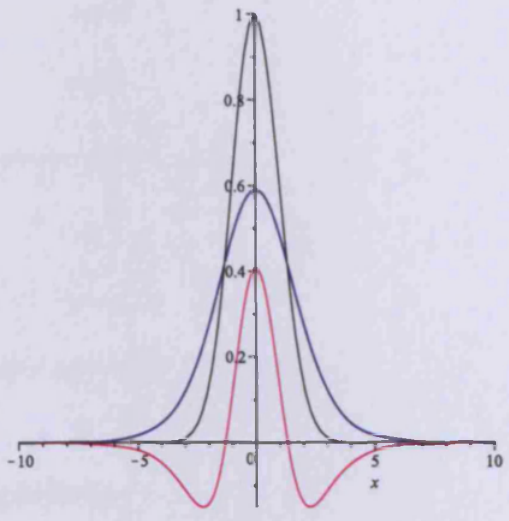

(b) $\sigma=1$

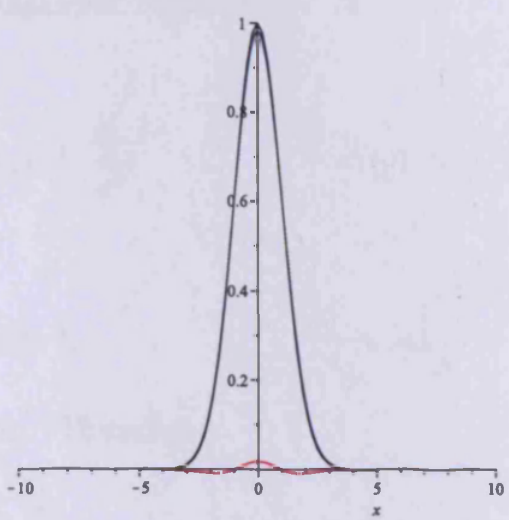

(d) $\sigma=\frac{1}{8}$

Figure 3.1: Gaussian decomposition. 


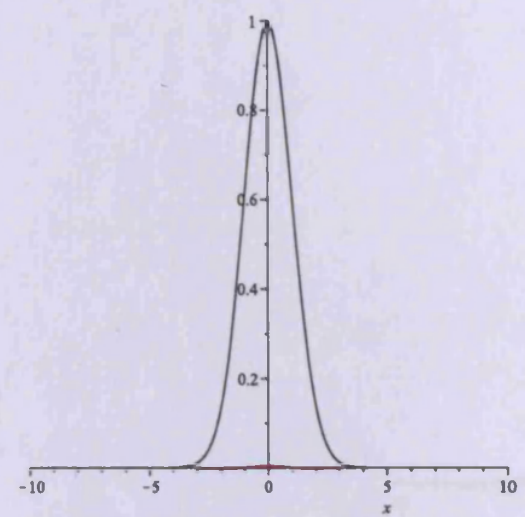

(a) $\sigma=\frac{1}{16}$

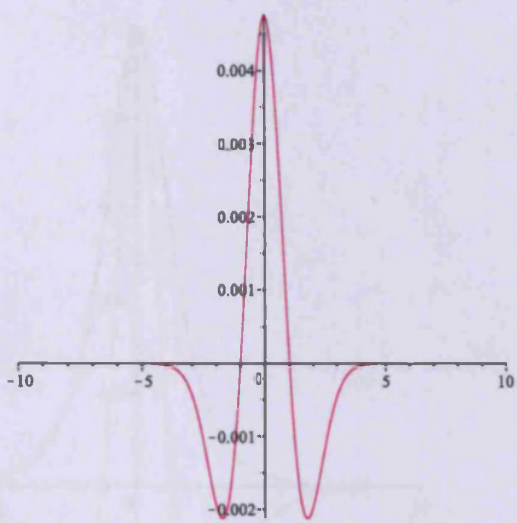

(b) Negligible Calderón part $\left(\sigma=\frac{1}{16}\right)$.

Figure 3.2: Gaussian decomposition.

The functions $(\phi \star \phi)(x)$ and $(\psi \star \psi)(x)$ are displayed together in Fig 3.3. From (3.2.10) and (2.1.11) I obtain

$$
h(t)=\frac{1}{\pi} g_{2}(t)+\frac{1}{\pi} \int_{0}^{1}\left(h \star \psi_{s} \star \psi_{s}\right)(t) \frac{d s}{s^{2}}
$$

which, in physical variables, reads

$$
H(\tau)=\frac{2}{\pi} G^{\prime \prime}\left(\tau^{-1}\right)+E(\tau)
$$

where $E(\tau)$ is the error in the FK approximation. Therefore

$$
E(\tau)=\frac{1}{\pi} \int_{0}^{1}\left(h \star \psi_{s} \star \psi_{s}\right)(t) \frac{d s}{s^{2}}
$$

I now form a representation for $E(\tau)$ in terms of the physical variables $\omega$ and $\tau$. Using equations (3.1.5) and (1.5.4), I find that

$$
\begin{aligned}
\left(\psi_{s} \star \psi_{s}\right)(t) & =\frac{1}{s} \int_{-\infty}^{\infty} \psi\left(\frac{t-x}{s}\right) \psi\left(\frac{x}{s}\right) d x \\
& =(\psi \star \psi)\left(\frac{t}{s}\right) .
\end{aligned}
$$




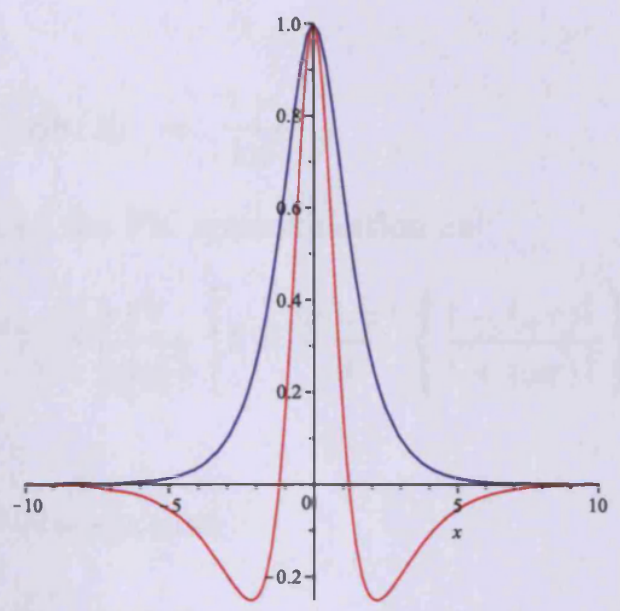

Figure 3.3: $(\psi \star \psi)(x)$ (red), $(\phi \star \phi)(x)$ (blue).

From (3.4.3), I obtain

$$
\left(\psi_{s} \star \psi_{s}\right)(t)=\operatorname{sech}\left(\frac{t}{s}\right)\left[1-\frac{t}{s} \tanh \left(\frac{t}{s}\right)\right] .
$$

By using (3.4.10), (1.5.4) and $\omega=\tau^{-1}$, I can now express the error term as

$$
\begin{aligned}
E(\tau)= & \frac{1}{\pi} \int_{0}^{1} h(t) \star \operatorname{sech}\left(\frac{t}{s}\right)\left[1-\frac{t}{s} \tanh \left(\frac{t}{s}\right)\right] \frac{d s}{s^{2}} \\
= & \frac{1}{\pi} \int_{0}^{1} \int_{-\infty}^{\infty} h(x) \operatorname{sech}\left(\frac{t-x}{s}\right)\left[1-\frac{t-x}{s} \tanh \left(\frac{t-x}{s}\right)\right] d x \frac{d s}{s^{2}} \\
= & \frac{1}{\pi} \int_{-\infty}^{\infty} \int_{0}^{1} H\left(\omega^{-1}\right) \operatorname{sech}\left(\ln \left[(\omega \tau)^{\frac{1}{s}}\right]\right) \\
& \times\left[1+\ln \left([\omega \tau]^{\frac{1}{s}}\right) \tanh \left(\ln [(\omega \tau)]^{-\frac{1}{s}}\right)\right] \frac{d s}{s^{2}} d(\ln \omega)
\end{aligned}
$$

Writing

$$
\operatorname{sech}(x)=\frac{2 x}{1+x^{2}}
$$


and

$$
\tanh (x)=\frac{1-x^{2}}{1+x^{2}}
$$

I can express the error term in the FK approximation as

$$
E(\tau)=\frac{2}{\pi} \int_{0}^{\infty} \int_{0}^{1} H\left(\omega^{-1}\right) \frac{(\omega \tau)^{\frac{1}{s}}}{1+(\omega \tau)^{\frac{2}{s}}}\left[1+\frac{\ln (\omega \tau)}{s}\left\{\frac{1-(\omega \tau)^{\frac{2}{3}}}{1+(\omega \tau)^{\frac{2}{s}}}\right\}\right] \frac{d s}{s^{2}} \frac{d \omega}{\omega}
$$

I note that equation (3.4.7) tells me that

$$
\int_{-\infty}^{\infty} E(\tau) d t=0
$$

which proves that

$$
\int_{0}^{\infty} E(\tau) \frac{d \tau}{\tau}=0
$$

This result is consistent with equation (3.4.6), since

$$
\int_{0}^{\infty} H(\tau) \frac{d \tau}{\tau}=\frac{2}{\pi} \int_{0}^{\infty} G^{\prime \prime}\left(\tau^{-1}\right) \frac{d \tau}{\tau}
$$

\subsection{Autoconvolutions and cross-pairings.}

It is conventional to interpret formula (3.2.10) as a means of reconstructing $h$ from its wavelet transforms $h \star \psi_{s}(0<s<\sigma)$ and $h \star \phi_{\sigma}$. In this interpretation $\psi$ and $\phi$ are mother-father pairs related through (3.1.17). The transforms are "coefficients" in the inversion formula, i.e. (3.2.10) may be written as

$$
h(t)=\frac{1}{C_{\psi}} \int_{0}^{\sigma}[W h](s, .) \star \psi_{s}(t) \frac{d s}{s^{2}}+\frac{1}{C_{\psi} \sigma}[V h](\sigma, .) \star \phi_{\sigma}(t) .
$$


Each of the two terms in (3.5.1) represents a projection of $h$ into subspaces of $L^{2}(\mathbb{R})$. If the scaling function $\phi$ is chosen appropriately then these two subspaces are complementary, i.e. non-overlapping. Discretizations of (3.5.1) then exist which lead to a multiresolution analysis (see Chui [25], Mallat [53]).

Since the storage and loss moduli give information at one scale only, namely $\sigma=1$, and the inversion formula (3.4.5) requires additional information at the smaller scales $0<s<1$, the formula cannot be used in a conventional way to recover $h(t)$. In this thesis I choose to interpret equation (3.2.10) in an alternative manner. This alternative approach involves pairing a mother wavelet from one family with a father wavelet from a different family. I shall call this approach cross-pairing. In particular I shall cross-pair the autoconvoluted mother wavelet $\psi \star \psi$ with the autoconvoluted father wavelet $\phi \star \phi$. The autoconvolutions $\psi \star \psi$ and $\phi \star \phi$ are not conventional mother-father pairs in general, since

$$
(\psi \star \psi \star \psi \star \psi)(x) \neq \frac{d}{d x}[x(\phi \star \phi \star \phi \star \phi)(x)] .
$$

The non-conventional relationship between $\psi \star \psi$ and $\phi \star \phi$ is illustrated below.

$$
\text { mother father }
$$

$A(x)$ is the father wavelet conventionally paired to the mother wavelet $\psi \star$ $\psi$, whereas $B(x)$ is the mother wavelet conventionally paired to the father 
wavelet $\phi \star \phi . A(x)$ is defined through its Fourier transform as

$$
\begin{aligned}
\widehat{A}(p) & =\left[\int_{p}^{\infty} \frac{\widehat{\psi}(\xi)^{4}}{\xi} d \xi\right]^{\frac{1}{2}} . \\
\text { Therefore } \quad A(x) & =\frac{1}{2 \pi} \int_{-\infty}^{\infty}\left[\int_{p}^{\infty} \frac{\widehat{\psi}^{4}(\xi)}{\xi} d \xi\right]^{\frac{1}{2}} e^{i p x} d p .
\end{aligned}
$$

$B(x)$ can be derived as follows

$$
\begin{aligned}
\widehat{\phi}^{4}(p) & =\int_{p}^{\infty} \frac{\widehat{B}^{2}(\xi)}{\xi} d \xi \\
\therefore \widehat{B}^{2}(p) & =-p \frac{d}{d p} \widehat{\phi}^{4}(p) \\
\therefore B(x) & =\frac{1}{2 \pi} \int_{-\infty}^{\infty}\left[-p \frac{d}{d p} \widehat{\phi}^{4}\right]^{\frac{1}{2}} e^{i p x} d p .
\end{aligned}
$$

In this alternative setting, I view equation (3.2.10) as a decomposition of $h(t)$ into constituent parts:

$$
h(t)=\frac{1}{C_{\psi}} \int_{0}^{\sigma}\left[W^{*} h\right](s, t) \frac{d s}{s^{2}}+\frac{1}{C_{\psi} \sigma}\left[V^{*} h\right](\sigma, t)
$$

where

$$
\left[W^{*} h\right](s, t)=\left(h \star \psi_{s} \star \psi_{s}\right)(t) \text { and }\left[V^{*} h\right](\sigma, t)=\left(h \star \phi_{\sigma} \star \phi_{\sigma}\right)(t) \text {. }
$$

The terms again represent projection of $h$ into $L^{2}(\mathbb{R})$, but the subspaces, in general, will overlap. In the context of this thesis an element of redundancy in the representation of $h(t)$ is not a matter for concern, given the lowdimensional nature of the approximations I shall use. Given the sensitivity of $h(t)$ to the choice of $\sigma$, an element of redundancy is actually an advantage, since it does not confine me to a rigid choice of orthogonal subspaces in the representation of $h$. 
I no longer insist that $\sigma=1$, but rather, choose $\sigma$ as a regularization parameter in the recovery process. In other words, $\sigma$ controls the strength of the low pass filter which provides me with a smoothed recovery of $h(t)$. High resolution terms will be confined to the first term in (3.5.5).

For ease of notation, I define

$$
\begin{aligned}
\phi^{\star}(x) & =(\phi \star \phi)(x), \\
\psi^{\star}(x) & =(\psi \star \psi)(x) .
\end{aligned}
$$

The cross-pairing I shall predominantly use is

$$
\begin{aligned}
\phi^{\star}(x) & =\operatorname{sech}(x) \\
\psi^{\star}(x) & =\operatorname{sech}(x)[1-x \tanh (x)] .
\end{aligned}
$$

I can now compare the mother wavelet pairings for $\phi^{\star}(x)=\operatorname{sech}(x)$ formed from the cross-pairing sense (3.5.10) with its mother wavelet pairing derived from the conventional Calderón-Mallat sense $(B(x))$, when $\sigma=1$. In the conventional sense,

$$
\begin{aligned}
B(x) \star B(x) & =\frac{d}{d x}\left[x\left(\phi^{\star} \star \phi^{\star}\right)(x)\right] \\
& =\frac{d}{d x}[x(\operatorname{sech} \star \operatorname{sech})(x)]
\end{aligned}
$$

In Chapter 4, it is proved that

$$
\operatorname{sech}(x) \star \operatorname{sech}(x)=2 x \operatorname{cosech}(x),
$$

which is displayed in Table 4.2 for $j=0$. Therefore, we obtain

$$
\begin{aligned}
B(x) \star B(x) & =\frac{d}{d x}\left[2 x^{2} \operatorname{cosech}(x)\right] \\
& =2 x \operatorname{cosech}(x)[2-x \operatorname{coth}(x)] .
\end{aligned}
$$

A closed form does not exist for $B(x)$, only for its auto-convolution, so I cannot directly compare $B(x)$ with $\psi^{\star}(x)$. I can, however, compare them in 
the Fourier domain. Taking the Fourier transform of both sides of equation (3.5.11), by using (2.1.13) and properties of the Fourier transform displayed in Table 1 , I find that

$$
\begin{aligned}
\widehat{B}^{2}(p) & =\left[\frac{d}{d x}(x[\operatorname{sech}(x) \star \operatorname{sech}(x)])\right]^{\wedge}(p) \\
& =-p \frac{d}{d p}[\operatorname{sech}(x) \star \operatorname{sech}(x)]^{\wedge}(p) \\
& =-p \frac{d}{d p}\left([\widehat{\operatorname{sech}}(p)]^{2}\right) \\
& =-\pi^{2} p \frac{d}{d p}\left[\operatorname{sech}^{2}\left(\frac{\pi}{2} p\right)\right] \\
& =\pi^{3} p \operatorname{sech}^{2}\left(\frac{\pi}{2} p\right) \tanh \left(\frac{\pi}{2} p\right) \\
\therefore \widehat{B}(p) & =\pi^{\frac{3}{2}} \operatorname{sech}\left(\frac{\pi}{2} p\right) \sqrt{p \tanh \left(\frac{\pi}{2} p\right)} .
\end{aligned}
$$

Using the relationship (3.1.17), the Fourier transform of $\psi^{\star}(x)$ can be derived as follows

$$
\begin{aligned}
\widehat{\psi^{\star}}(p) & =\left[\frac{d}{d x}(x \operatorname{sech}(x))\right]^{\wedge}(p) \\
& =-p \frac{d}{d p} \widehat{\operatorname{sech}(p)} \\
& =-\pi p \frac{d}{d p} \operatorname{sech}\left(\frac{\pi}{2} p\right) \\
& =\frac{\pi^{2}}{2} p \operatorname{sech}\left(\frac{\pi}{2} p\right) \tanh \left(\frac{\pi}{2} p\right)
\end{aligned}
$$

The two functions (3.5.15) and (3.5.16) are plotted against each other in Fig. 3.4 , where they have both been normalized in the sense that they have unit area. The two mother wavelets, when plotted in Fourier space have a very similar shape, but have their maxima at slightly different locations. 


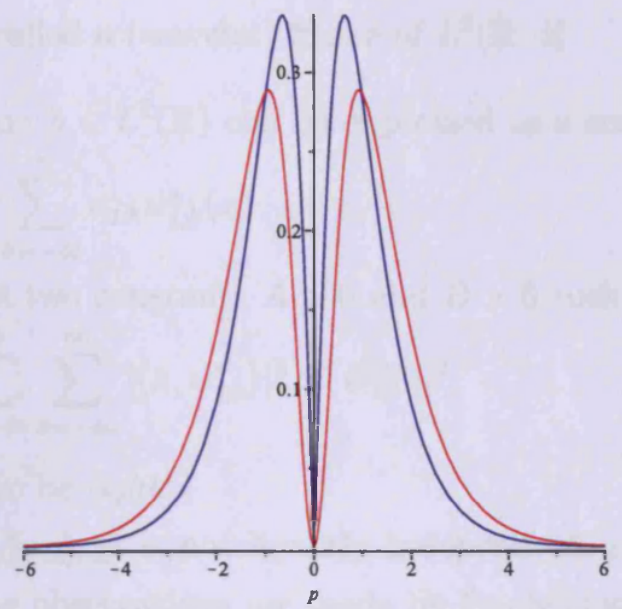

Figure 3.4: $\widehat{B}(p)$ (blue) against $\widehat{\psi^{\star}}(p)$ (red).

\subsection{Wavelet frames and Riesz bases.}

\subsubsection{Frames}

Consider discrete values of the scale $\sigma$ given by $\sigma=2^{-j}(j \in \mathbb{Z})$, and translations which are integer multiples of a sampling rate $a \in \mathbb{R}$. This gives rise to the set of vectors

$$
\psi_{j, k}^{\star}(x)=2^{\frac{j}{2}} \psi^{\star}\left(2^{j} x-k a\right)
$$

where the sampling rate $a$ is a free parameter. 
The set $\left\{\psi_{j, k}^{\star}\right\}_{(j, k) \in \mathbf{Z}^{2}}$ is called a (wavelet) frame of $L^{2}(\mathbb{R})$ if

(i) any function $h \in L^{2}(\mathbb{R})$ can be expressed as a series

$$
h(x)=\sum_{j=-\infty}^{\infty} \sum_{k=-\infty}^{\infty} a_{j, k} \psi_{j, k}^{\star}(x)
$$

and (ii) there exist two constants $A>0$ and $B>0$ such that

$$
A\|h\|^{2} \leq \sum_{j=-\infty}^{\infty} \sum_{k=-\infty}^{\infty}\left|\left\langle h, \psi_{j, k}^{\star}\right\rangle\right|^{2} \leq B\|h\|^{2} .
$$

If $A=B$ the frame is said to be tight.

In general, the set $\left\{\psi_{j, k}^{\star}\right\}_{(j, k) \in \mathbf{Z}^{2}}$ is not linearly independent, even if the frame is tight. The following observations are made by Daubechies [26] and Mallat [53]. To be linearly independent, the set must define a Riesz basis in $L^{2}(\mathbb{R})$. I shall consider Riesz bases in the next section. A set which is not linearly independent is said to be redundant. A measure of redundancy is given by the size of the frame bounds $A$ and $B$. The frame is redundant if $A>1$. The frame is orthonormal if and only if $A=B=1$. If the set is a normalised Riesz basis then the frame bounds satisfy $A \leq 1 \leq B$.

Daubechies [26] has given a sufficient condition for $\left\{\psi_{j, k}^{\star}\right\}_{(j, k) \in \mathbf{Z}^{2}}$ to be a frame. Let

$$
\begin{aligned}
\beta(\xi) & =\sup _{1 \leq|\omega| \leq 2} \sum_{j=-\infty}^{\infty}\left|\widehat{\psi^{\star}}\left(2^{j} \omega\right)\right|\left|\widehat{\psi^{\star}}\left(2^{j} \omega+\xi\right)\right| \\
\text { and } \quad \Delta & =\sum_{\substack{k=-\infty \\
k \neq 0}}^{\infty}\left[\beta\left(\frac{2 \pi k}{a}\right) \beta\left(-\frac{2 \pi k}{a}\right)\right]^{\frac{1}{2}} .
\end{aligned}
$$

If the sampling rate $a$ and scale $\left(2^{-j}\right)$ are such that

$$
\begin{aligned}
A_{0} & =\frac{1}{a}\left[\left.\inf _{1 \leq|\omega| \leq 2} \sum_{j=-\infty}^{\infty} \widehat{\psi^{\star}}\left(2^{j} \omega\right)\right|^{2}-\Delta\right]>0 \\
\text { and } \quad B_{0} & =\frac{1}{a}\left[\left.\sup _{1 \leq|\omega| \leq 2} \sum_{j=-\infty}^{\infty} \widehat{\psi^{\star}}\left(2^{j} \omega\right)\right|^{2}+\Delta\right]<\infty,
\end{aligned}
$$


then $\left\{\psi_{j, k}^{\star}\right\}_{(j, k) \in \mathbb{Z}^{2}}$ is a frame of $L^{2}(\mathbb{R})$.

The constants $A_{0}$ and $B_{0}$ are respectively lower and upper bounds of the frame bounds $A$ and $B$. If $B_{0} / A_{0} \approx 1$ then it follows that the frame is almost tight. If we work with a redundant frame $(1<A \leq B)$ then the representation (3.6.2) is not unique, and the representation will depend upon the choice of algorithm used to compute the coefficients.

\subsubsection{Hyperbolic and Gaussian wavelet frames}

One of the benchmark wavelets which defines a frame is the well known Mexican hat wavelet $M(x)$, defined as the second derivative of a Gaussian with unit standard deviation, i.e.

$$
\begin{aligned}
M(x) & =\frac{d^{2}}{d x^{2}} e^{-\frac{x^{2}}{2}} \\
& =\left(x^{2}-1\right) e^{-\frac{x^{2}}{2}}
\end{aligned}
$$

On the other hand, I have found nothing in the literature concerning whether hyperbolic wavelets of the form $\phi^{\star}$ and $\psi^{\star}$ can be used to generate frames. Motivated by the important links with linear viscoelasticity that I have already demonstrated, I shall in this section establish some key results on which the work in this thesis depends.

Definitions. Let $\boldsymbol{\Psi}$ denote the set $\left\{\psi_{j, k}^{\star}\right\}_{(j, k) \in \mathbf{Z}^{2}}$, where

$$
\psi^{\star}(x)=\operatorname{sech}(x)[1-x \tanh (x)]
$$

Let $\mathbf{B}$ denote the set $\left\{B_{j, k}\right\}_{(j, k) \in \mathbb{Z}^{2}}$, where

$$
\begin{aligned}
B(x) \star B(x) & =\frac{d}{d x}\left[2 x^{2} \operatorname{cosech}(x)\right] \\
& =2 x \operatorname{cosech}(x)[2-x \operatorname{coth}(x)] .
\end{aligned}
$$


Let $\mathbf{M}$ denote the set $\left\{M_{j, k}\right\}_{(j, k) \in \mathbb{Z}^{2}}$ where $M(x)$ is given by (3.6.8). The basic wavelets and their corresponding Fourier transforms, which generate the sets $\mathbf{\Psi}, \mathbf{B}$ and $\mathbf{M}$ are shown in Fig. 3.5, with normalizations $\left\|\psi^{\star}\right\|_{2}=$ $\|B\|_{2}=\|M\|_{2}=1$.

Theorem 3.2. The sets $\Psi, \mathbf{B}$ and $\mathbf{M}$ each define frames of $L^{2}(\mathbb{R})$ for a sampling rate $a \leq 2$.

Theorem 3.3. With a sampling rate $a \leq 1$, frame $\mathbf{B}$ is tighter than frame $\Psi$, which is tighter than frame $\mathbf{M}$.

Remark: Theorem 3.2 is also true for certain sample rates $a>2$. In particular, there exist threshold values of $a$, namely $a_{\psi}>2, a_{B}>2$ and $a_{M}>2$, for which

(i) $\boldsymbol{\Psi}$ is a frame for all $0<a<a_{\psi}$;

(ii) $\mathbf{B}$ is a frame for all $0<a<a_{B}$;

(iii) $\mathbf{M}$ is a frame for all $0<a<a_{M}$.

We have used Daubechies' necessary and sufficient conditions (equations (3.64)-(3.67)) to calculate the frame bounds for $\mathbf{\Psi}, \mathbf{B}$ and $\mathbf{M}$, for certain values of the sampling rates, and with normalisations $\left\|\psi^{\star}\right\|_{2}=\|B\|_{2}=$ $\|M\|_{2}=1$. These are shown in Tables 3.1, 3.2 and 3.3. Theorems 3.2 and 3.3 are now easily established by viewing the frame bounds shown in these tables. 


\begin{tabular}{|c|c|c|c|}
\hline sampling rate $(a)$ & $A_{0}$ & $B_{0}$ & $B_{0} / A_{0}$ \\
\hline 2 & 1.363 & 3.298 & 2.421 \\
\hline $3 / 2$ & 2.718 & 3.497 & 1.289 \\
\hline 1 & 4.616 & 4.706 & 1.020 \\
\hline $1 / 2$ & 9.313 & 9.331 & 1.002 \\
\hline $1 / 4$ & 18.626 & 18.662 & 1.002 \\
\hline
\end{tabular}

Table 3.1: Estimated frame bounds for the hyperbolic wavelet $\psi_{j, k}^{\star}(x)$.

\begin{tabular}{|c|c|c|c|}
\hline sampling rate $(a)$ & $A_{0}$ & $B_{0}$ & $B_{0} / A_{0}$ \\
\hline 2 & 5.551 & 8.688 & 1.565 \\
\hline $3 / 2$ & 8.974 & 10.011 & 1.116 \\
\hline 1 & 14.194 & 14.283 & 1.006 \\
\hline $1 / 2$ & 28.471 & 28.485 & 1.0005 \\
\hline $1 / 4$ & 56.941 & 56.969 & 1.0005 \\
\hline
\end{tabular}

Table 3.2: Estimated frame bounds for the hyperbolic wavelet $B_{j, k}(x)$.

\subsubsection{Riesz bases.}

$$
\text { Let } \quad V_{0}=\operatorname{clos}_{L^{2}(\mathbf{R})} \operatorname{span}\left\{\phi_{0, k}^{\star}: k \in \mathbb{Z}\right\}
$$

The set $\left\{\phi_{0, k}^{\star}(x)\right\}$ is said to be a Riesz basis of $V_{0}$ if it is linearly independent and there exist constants $C>0$ and $D>0$ such that for any $y \in V_{0}$ there is an unique series representation

$$
h(x)=\sum_{k=-\infty}^{\infty} b_{k} \phi_{0, k}^{\star}(x)
$$




\begin{tabular}{|c|c|c|c|}
\hline sampling rate $(a)$ & $A_{0}$ & $B_{0}$ & $B_{0} / A_{0}$ \\
\hline 2 & 1.248 & 2.162 & 1.733 \\
\hline $3 / 2$ & 2.133 & 2.413 & 1.131 \\
\hline 1 & 3.273 & 3.546 & 1.084 \\
\hline $1 / 2$ & 6.545 & 7.092 & 1.084 \\
\hline $1 / 4$ & 13.091 & 14.183 & 1.084 \\
\hline
\end{tabular}

Table 3.3: Estimated frame bounds for the Mexican hat wavelet $M(x)$.

which satisfies

$$
C\|y\|^{2} \leq \sum_{k=-\infty}^{\infty}\left|b_{k}\right|^{2} \leq D\|y\|^{2} .
$$

Mallat [53] shows that the inequalities (3.6.13) are equivalent to the following inequalities on the Fourier transform of $\phi^{\star}$

$$
\forall \omega \in[-\pi, \pi], \quad \frac{1}{D} \leq \sum_{k=-\infty}^{\infty}\left|\widehat{\phi^{\star}}(\omega-2 k \pi)\right|^{2} \leq \frac{1}{C} .
$$

Theorem 3.4. The set $\left\{\phi_{0, k}^{\star}\right\}_{k \in \mathbf{Z}}$, where

$$
\phi^{\star}(x)=\operatorname{sech}(x)
$$

is a Riesz basis of the space $V_{0}$ it generates, since

$$
\forall \omega \in[-\pi, \pi], \quad 0.000414 \leq \sum_{k=-\infty}^{\infty}\left|\widehat{\phi^{\star}}(\omega-2 k \pi)\right|^{2} \leq 1.00000003 .
$$

The basis has been normalised in the sense that $\left\|\phi_{0, k}^{\star}\right\|=1$.

Similarly, I define

$$
\begin{aligned}
V_{J} & =\operatorname{clos}_{L^{2}(\mathbf{R})} \operatorname{span}\left\{\phi_{J, k}^{\star}: k \in \mathbb{Z}\right\}, \quad J \in \mathbb{Z}, \\
\text { where } \phi_{J, k}^{\star}(x) & =2^{\frac{J}{2}} \phi^{\star}\left(2^{J} x-k a\right)
\end{aligned}
$$





(a) Mexican Hat wavelet (blue) (b) Fourier space comparison. $\widehat{B}(p)$ against $\psi^{\star}(x)$ (red) (blue), $\widehat{\psi^{\star}}(p)$ (red) and $\widehat{M}(p)$ (black).

Figure 3.5: Wavelet comparison.

The set $\left\{\phi_{J, k}^{\star}\right\}_{k \in \mathbb{Z}}$ is a Riesz basis of the space $V_{J} \subset L^{2}(\mathbb{R})$. Furthermore, I define

$$
\begin{aligned}
W_{j} & =\operatorname{clos}_{L^{2}(\mathbb{R})} \operatorname{span}\left\{\psi_{j, k}^{\star}: k \in \mathbb{Z}\right\}, \quad j \in \mathbb{Z}, \\
\text { where } \psi_{j, k}^{\star}(x) & =2^{\frac{j}{2}} \psi^{\star}\left(2^{j} x-k a\right) .
\end{aligned}
$$

The set $\left\{\psi_{j, k}^{\star}\right\}_{k \in \mathbb{Z}}$ is also a Riesz basis of the space $W_{j} \subset L^{2}(\mathbb{R})$.

It is now clear that a discrete representation of the Calderón-Mallat decomposition exists in the form

$$
h(x)=\sum_{j} \sum_{k} a_{j, k} \psi_{j, k}^{\star}(x)+\sum_{k} b_{J, k} \phi_{J, k}^{\star}(x) .
$$

Given $J$, the coefficient set $\left\{b_{J, k}\right\}$ is uniquely determined, whereas the double series constituting the first term is not uniquely determined. 


\subsection{Multiresolution analysis (MRA) and two- scale relations.}

A scaling function $\phi$ and its sequence of associated subspaces

$$
V_{j}=\cos _{L^{2}(\mathbf{R})} \operatorname{span}\left\{\phi_{j, k}: k \in \mathbb{Z}\right\}, \quad j \in \mathbb{Z},
$$

are said to generate a multiresolution analysis if

$$
f(x) \in V_{j} \Leftrightarrow f(2 x) \in V_{j+1}, \quad j \in \mathbb{Z},
$$

and

1. the subspaces are nested, i.e. $V_{j} \subset V_{j+1}$;

2. the union of the subspaces is dense in $L^{2}(\mathbb{R})$;

3.

$$
\bigcap_{j \in \mathbf{Z}} V_{j}=\{0\}
$$

4. there can be found a wavelet $\psi$ and a corresponding sequence of subspaces $W_{j}=\operatorname{clos}_{L^{2}(\mathbf{R})} \operatorname{span}\left\{\psi_{j, k}: k \in \mathbb{Z}\right\}, j \in \mathbb{Z}$, such that $V_{j+1}=V_{j} \oplus W_{j}$, where $\oplus$ denotes a direct sum.

This means that a first approximation $f_{J}$ to a function $f \in L^{2}(\mathbb{R})$ may be found by projecting $f$ onto the subspace $V_{J}$, and improved approximations may then be found by adding to the $f_{J}$ suitable contributions at finer scales from the subspaces $W_{J}, W_{J+1}, W_{J+2}, \ldots$, i.e.

$$
f=f_{J}+g_{J}+g_{J+1}+g_{J+2}+\ldots
$$

If the subspaces $W_{j}$ are orthogonal then the decomposition (3.7.3) is unique, otherwise the functions $g_{j}$ are not uniquely defined. This means that I can 
modify equation (3.6.21) to cut off all scales $\sigma>2^{-J}$ in the mother wavelet part. I then obtain a representation for $h(t)$ which more closely resembles a discrete form of the Calderón-Mallat decomposition

$$
h(x)=\sum_{\substack{j \\ j \geq J}} \sum_{k} a_{j, k} \psi_{j, k}^{\star}(x)+\sum_{k} b_{J, k} \phi_{J, k}^{\star}(x) .
$$

In a multiresolution analysis it is clear that $\phi \subset V_{0}, \psi \subset W_{0}, V_{0} \subset V_{1}$ and $W_{0} \subset V_{1}$. Consequently there exist relationships between $\phi(x)$ and $\phi(2 x)$, and between $\psi(x)$ and $\phi(2 x)$, of the form

$$
\begin{aligned}
& \phi(x)=\sum_{k} a_{k} \phi(2 x-k), \\
& \psi(x)=\sum_{k} b_{k} \phi(2 x-k) .
\end{aligned}
$$

These are called two-scale relations, and play an important role in synthesizing wavelet reconstructions in signal processing.

Taking the Fourier transform of both sides of (3.7.5), I obtain

$$
\begin{aligned}
\widehat{\phi}(p) & =\frac{1}{2} \sum_{k} a_{k} \widehat{\phi}\left(\frac{p}{2}\right) e^{-i \frac{k}{2} p} \\
\therefore \frac{\widehat{\phi}(p)}{\widehat{\phi}\left(\frac{p}{2}\right)} & =\frac{1}{2} \sum_{k} a_{k} e^{-i \frac{k}{2} p} .
\end{aligned}
$$

This requires that $\frac{\widehat{\phi}(p)}{\hat{\phi}\left(\frac{p}{2}\right)}$ has compact support on $[-2 \pi, 2 \pi]$. Similarly, from (3.7.6), I derive

$$
\frac{\widehat{\psi}(p)}{\widehat{\phi}\left(\frac{p}{2}\right)}=\frac{1}{2} \sum_{k} b_{k} e^{-i \frac{k}{2} p}
$$

which requires $\frac{\hat{\psi}(p)}{\hat{\phi}\left(\frac{p}{2}\right)}$ to have compact support on $[-2 \pi, 2 \pi]$. The cross-paired wavelets $\left\{\psi^{\star}, \phi^{\star}\right\}$, defined by (3.6.9) and (3.6.15) do not satisfy the two-scale relationships (3.7.5) and (3.7.6), since

$$
\frac{\widehat{\phi^{\star}}(p)}{\widehat{\phi}^{\star}\left(\frac{p}{2}\right)}=\frac{\operatorname{sech}\left(\frac{\pi}{2} p\right)}{\operatorname{sech}\left(\frac{\pi}{4} p\right)}
$$


does not have compact support on $[-2 \pi, 2 \pi]$, which is illustrated in Fig. 3.6. Similarly,



Figure 3.6: $\frac{\widehat{\phi^{*}}(p)}{\phi^{\star}\left(\frac{p}{2}\right)}$ does not have compact support on $[-2 \pi, 2 \pi]$.

$$
\frac{\widehat{\psi^{\star}}(p)}{{\widehat{\phi^{\star}}}_{\left(\frac{p}{2}\right)}}=\frac{\pi p \operatorname{sech}\left(\frac{\pi}{2} p\right) \tanh \left(\frac{\pi}{2} p\right)}{\operatorname{sech}\left(\frac{\pi}{4} p\right)}
$$

does not have compact support on $[-2 \pi, 2 \pi]$, which is shown in Fig. 3.7. Nevertheless, the cross-paired wavelets $\left\{\psi^{\star}, \phi^{\star}\right\}$, defined by (3.6.9) and (3.6.15), do satisfy equations (3.7.5) and (3.7.6) in an approximate sense. In particular, I will show that if the coefficients $\left\{a_{k}, b_{k}\right\}$ are chosen as the Fourier coefficients

$$
\begin{aligned}
& a_{k}=\frac{1}{2 \pi} \int_{-2 \pi}^{2 \pi} \frac{\widehat{\phi^{\star}}(p)}{\widehat{\phi}^{\star}\left(\frac{p}{2}\right)} e^{\frac{i k p}{2}} d p, \\
& b_{k}=\frac{1}{2 \pi} \int_{-2 \pi}^{2 \pi} \frac{\widehat{\psi^{\star}}(p)}{\widehat{\phi^{\star}}\left(\frac{p}{2}\right)} e^{\frac{i k p}{2}} d p,
\end{aligned}
$$






Figure 3.7: $\frac{\widehat{\psi^{*}}(p)}{\phi^{*}\left(\frac{p}{2}\right)}$ does not have compact support on $[-2 \pi, 2 \pi]$.

then, although the residuals in the two-scale relations will not tend to zero as $k \rightarrow \infty$, they will be extremely small. Since equations (3.7.5) and (3.7.6) are not satisfied exactly, I shall calculate estimates on the error functions $\epsilon(x)$ and $\rho(x)$, where

$$
\epsilon(x)=\phi^{\star}(x)-\sum_{k} a_{k} \phi^{\star}(2 x-k)
$$

and

$$
\rho(x)=\psi^{\star}(x)-\sum_{k} b_{k} \phi^{\star}(2 x-k) .
$$

Theorem 3.5.

$$
\|\epsilon\|_{2}<5 \times 10^{-4} \text { and }\|\rho\|_{2}<2 \times 10^{-3} .
$$

Alternatively, using best approximation in the supremum norm,

$$
\|\epsilon\|_{\infty}<5 \times 10^{-4} \text { and }\|\rho\|_{\infty}<2 \times 10^{-3} .
$$


Since the two-scale relations only hold exactly in the range $-2 \pi<p<2 \pi$, I obtain

$$
\widehat{\epsilon}(p)=\left\{\begin{array}{l}
\widehat{\phi^{\star}}(p)-\frac{1}{2} \widehat{\phi^{\star}}\left(\frac{p}{2}\right) \sum_{k} a_{k} e^{-i \frac{k}{2} p}, \quad-\infty<p<\infty ; \\
0, \quad-2 \pi<p<2 \pi .
\end{array}\right.
$$

For $|p|>2 \pi$ I can express $\widehat{\epsilon}(p)$ as follows, by splitting the real line into intervals of length $4 \pi$. So $p \in[-2 \pi+4 \pi n, 2 \pi+4 \pi n], n=-\infty \ldots \infty$. When $n=0$ we get the above interval $[-2 \pi, 2 \pi]$. I observe that $\widehat{\epsilon}(p)=\widehat{\epsilon}(q+4 \pi n)$, $q \in[-2 \pi, 2 \pi]$. Thus, I now have

$\widehat{\epsilon}(p)=\left\{\begin{array}{l}0, \quad-2 \pi<p<2 \pi \\ \sum_{n}\left[\widehat{\phi^{\star}}(p+4 \pi n)-\frac{1}{2} \widehat{\phi^{\star}}\left(\frac{p}{2}+2 \pi n\right) \sum_{k} a_{k} e^{-i \frac{k}{2} p}\right], \quad|p|>2 \pi,\end{array}\right.$

where I have used the fact that $e^{-i \frac{k}{2}(p+4 \pi n)}=e^{-i \frac{k}{2} p}$. By Parseval's theorem $[53]$,

$$
\|\epsilon\|_{2}^{2}=\frac{1}{2 \pi}\|\widehat{\epsilon}\|_{2}^{2}
$$

Consequently, using (3.7.17) and (3.7.18), I obtain

$$
\begin{aligned}
\|\epsilon\|_{2}^{2} & =\frac{1}{2 \pi} \int_{-2 \pi}^{2 \pi}\left\{\sum_{n=-\infty}^{n=\infty}\left[\widehat{\phi^{\star}}(p+4 \pi n)-\frac{1}{2} \widehat{\phi^{\star}}\left(\frac{p}{2}+2 \pi n\right) \sum_{k} a_{k} e^{-i \frac{k}{2} p}\right]\right\}^{2} d p \\
& =\frac{1}{2 \pi} \sum_{n=-\infty}^{n=\infty} \int_{-2 \pi}^{2 \pi}\left[\widehat{\phi^{\star}}(p+4 \pi n)-\widehat{\phi}^{\star}\left(\frac{p}{2}+2 \pi n\right) \frac{\widehat{\phi}^{\star}(p)}{\widehat{\phi}^{\star}\left(\frac{p}{2}\right)}\right]^{2} d p, \quad(3.7 .20)
\end{aligned}
$$

using (3.7.7). When $n=0$, the integrand is equal to zero. Also, when $|n|>1$, the integrand is so small that there is a negligible contribution to the sum. This is because $\widehat{\phi^{\star}}(p)$ is very small for large $|p|$. For $\phi^{\star}(x)=\operatorname{sech}(x)$, $\|\epsilon\|_{2}^{2}=\frac{\pi}{2} \sum_{n=-\infty}^{n=\infty} \int_{-2 \pi}^{2 \pi}\left[\operatorname{sech}\left(\frac{\pi}{2}[p+4 \pi n]\right)-\operatorname{sech}\left(\frac{\pi}{4}[p+4 \pi n]\right) \frac{\operatorname{sech}\left(\frac{\pi}{2} p\right)}{\operatorname{sech}\left(\frac{\pi}{4} p\right)}\right]^{2} d p$. 
Similarly, I obtain

$$
\widehat{\rho}(p)=\left\{\begin{array}{l}
0, \quad-2 \pi<p<2 \pi \\
\sum_{n}\left[\widehat{\psi^{\star}}(p+4 \pi n)-\frac{1}{2} \widehat{\phi}^{\star}\left(\frac{p}{2}+2 \pi n\right) \sum_{k} b_{k} e^{-i \frac{k}{2} p}\right], \quad|p|>2 \pi
\end{array}\right.
$$

and consequently,

$$
\|\rho\|_{2}^{2}=\frac{1}{2 \pi} \sum_{n=-\infty}^{\infty} \int_{-2 \pi}^{2 \pi}\left[\widehat{\psi^{\star}}(p+4 \pi n)-\widehat{\phi}^{\star}\left(\frac{p}{2}+2 \pi n\right) \frac{\widehat{\psi^{\star}}(p)}{\widehat{\phi}^{\star}\left(\frac{p}{2}\right)}\right]^{2} d p .
$$

For $\psi^{\star}(x)=\operatorname{sech}(x)[1-x \tanh (x)]$, I find that

$$
\begin{aligned}
\|\rho\|_{2}^{2}= & \frac{\pi^{3}}{8} \sum_{n=-\infty}^{n=\infty} \int_{-2 \pi}^{2 \pi}\left\{(p+4 \pi n) \operatorname{sech}\left(\frac{\pi}{2}[p+4 \pi n]\right) \tanh \left(\frac{\pi}{2}[p+4 \pi n]\right)\right. \\
& \left.-\operatorname{sech}\left(\frac{\pi}{4}[p+4 \pi n]\right) \frac{p \operatorname{sech}\left(\frac{\pi}{2} p\right) \tanh \left(\frac{\pi}{2} p\right)}{\operatorname{sech}\left(\frac{\pi}{4} p\right)}\right\}^{2} d p .
\end{aligned}
$$

The series in (3.7.21) and (3.7.24) converge very rapidly, and by calculating the integrals numerically, it can be shown that

$$
\|\epsilon\|_{2}<5 \times 10^{-4} \text { and }\|\rho\|_{2}<2 \times 10^{-3} \text {. }
$$

Alternatively, using best approximation in the supremum norm it can be shown that

$$
\|\epsilon\|_{\infty}<5 \times 10^{-4} \text { and }\|\rho\|_{\infty}<2 \times 10^{-3} \text {. }
$$

The coefficient set $\left\{a_{k}, b_{k}\right\}$ is displayed in Table 3.4 for both of the above cases. By viewing Table 3.4 , it is clear that the coefficients $a_{k}$ compare relatively well, but the coefficients $b_{k}$ have a slightly larger discrepancy. At finer scales $\sigma=2^{-j}, j>0$, the $L^{2}$ norms of the residuals are reduced, since

$$
\left\|\epsilon\left(2^{j} x\right)\right\|_{2}<5 \times 2^{-\frac{j}{2}} \times 10^{-4} \text { and }\left\|\rho\left(2^{j} x\right)\right\|_{2}<2 \times 2^{-\frac{j}{2}} \times 10^{-3} .
$$


However, there is no reduction in the supremum norms (3.7.26). The first inequality in (3.7.27) is proved using the following argument

$$
\begin{aligned}
\left\|\epsilon\left(2^{j} x\right)\right\|_{2}= & \left(\int_{-\infty}^{\infty}\left[\epsilon\left(2^{j} x\right)\right]^{2} d x\right)^{\frac{1}{2}} \\
= & \left(\int_{-\infty}^{\infty} 2^{-j}[\epsilon(X)]^{2} d X\right)^{\frac{1}{2}} \quad\left(X=2^{j} x\right) \\
= & 2^{-\frac{j}{2}}\left(\int_{-\infty}^{\infty}[\epsilon(x)]^{2} d x\right)^{\frac{1}{2}} \quad(x=X) \\
= & 2^{-\frac{j}{2}}\|\epsilon(x)\|_{2} \\
& <5 \times 2^{-\frac{j}{2}} \times 10^{-4}
\end{aligned}
$$

The other inequality is proved in the same fashion.

In working with experimental data, the storage and loss moduli are rarely given to an accuracy of less than $1 \%$. Consequently, the choice of an approximate multiresolution analysis with scaling $\sigma \leq 4$ is more than adequate when working with a cross-pair $\left\{\phi^{\star}, \psi^{\star}\right\}$.

\subsection{Choice of primary subspace}

In order to give a wider choice of base subspace, which I call the primary subspace, I no longer insist upon the dyadic scaling $\sigma=2^{-j}$ for the scaling functions. I now allow resolutions of the form

$$
\begin{aligned}
\sigma & =2^{-j} \sigma_{0}, \quad j \in \mathbb{Z}, \sigma_{0} \in \mathbb{R} . \\
\text { Then } \phi_{j, k}^{\star}(x) & =\phi^{\star}\left(2^{j} \sigma_{0}^{-1} x-k a \sigma_{0}^{-1}\right), \\
\text { where } \phi^{\star}(x) & =\operatorname{sech}\left(\frac{x}{\sigma_{0}}\right)
\end{aligned}
$$

and $a \sigma_{0}^{-1}$ is the new sampling rate. $\sigma_{0}$ is a non-dyadic control on the scale in the primary subspace. The primary subspace can be kept as $V_{0}$ by varying $\sigma_{0}$ accordingly (i.e. $\sigma=\sigma_{0}$ ). 


\begin{tabular}{|c|c|c|c|c|}
\hline$k$ & $a_{k}\left(L^{2}\right)$ & $b_{k}\left(L^{2}\right)$ & $a_{k}$ (Minimax) & $b_{k}$ (Minimax) \\
\hline 0 & 0.44724 & 0.76911 & 0.44417 & 0.75786 \\
\hline 1 & 0.33103 & 0.25317 & 0.33440 & 0.26495 \\
\hline 2 & 0.18352 & -0.10911 & 0.17949 & -0.12232 \\
\hline 3 & 0.10581 & -0.12672 & 0.11032 & -0.11188 \\
\hline 4 & 0.06163 & -0.11473 & 0.05680 & -0.13059 \\
\hline 5 & 0.03746 & -0.08330 & 0.04245 & -0.06668 \\
\hline 6 & 0.02228 & -0.06426 & 0.01718 & -0.08134 \\
\hline 7 & 0.01375 & -0.04362 & 0.01890 & -0.02618 \\
\hline 8 & 0.00814 & -0.03210 & 0.00295 & -0.04977 \\
\hline 9 & 0.00509 & -0.02075 & 0.01030 & -0.00291 \\
\hline 10 & 0.00296 & -0.01508 & -0.00225 & -0.03301 \\
\hline
\end{tabular}

Table 3.4: Coefficients determined by an $L^{2}$ algorithm (Fourier coefficients) and by Minimax.

\subsection{Conclusion}

The main purpose of this chapter was to introduce wavelet analysis and the concept that wavelet transforms arise naturally in the theory of linear viscoelasticity. I have shown that the loss modulus is in fact a father wavelet transform of the spectrum at unit scaling in log-time and I have used wavelet analysis to validate expressing the continuous relaxation spectrum as a sum of wavelets.

I have introduced the Calderón-Mallat decomposition of the identity, and used it to calculate the error term in one of the earliest approximations of the continuous relaxation spectrum. I have also defined a new way of pairing mother and father wavelets from different families, which I call cross-pairing. In particular, I have shown that the mother and father wavelet cross-pairing 
$\left(\psi^{\star}(x), \phi^{\star}(x)\right)=(\operatorname{sech}(x)[1-x \tanh (x)], \operatorname{sech}(x))$ satisfies the respective rigorous constraints of wavelet frames and Riesz bases. In particular, it has been shown that the mother wavelets associated with $\operatorname{sech}(x)$, in both the conventional Calderón-Mallat sense and the cross-pairing method, have tighter frames than the well-known Mexican hat wavelet for frame sampling rates $a \leq 1$. Furthermore, it has been shown that these cross-paired wavelets can be used to construct an essential multiresolution analysis. 
CHAPTER 3. 


\section{Chapter 4}

\section{Model Development}

\subsection{Real-Time Integrable (RTI) Wavelets}

It is my purpose in this thesis to represent the continuous relaxation spectrum in logarithmic time as a sum of wavelets, i.e.

$$
h(t)=\sum_{\substack{j \\ j \geq J}} \sum_{k} a_{j, k} \psi_{j, k}^{\star}\left(t-t_{k}\right)+\sum_{k} b_{J, k} \phi_{J, k}^{\star}\left(t-t_{k}\right) .
$$

With the choice

$$
\phi^{\star}(t)=\operatorname{sech}\left(\frac{t}{\sigma}\right), \quad \psi^{\star}(t)=\operatorname{sech}\left(\frac{t}{\sigma}\right)\left[1-\frac{t}{\sigma} \tanh \left(\frac{t}{\sigma}\right)\right],
$$

in this first section I address the physical constraints imposed on the parameter $\sigma$, and the procedures I must follow to satisfy these constraints.

Every viscoelastic material possesses a finite total viscosity. This imposes an integral constraint on $H(\tau)$ and $h(t)$, given by

$$
\int_{0}^{\infty} H(\tau) d \tau=\int_{-\infty}^{\infty} h(t) e^{-t} d t<\infty
$$

I define $h$ to be real-time integrable (RTI) if $h$ satisfies constraint (4.1.3). The representation of $h$ in (4.1.1) consequently demands that both the father 
wavelet $\phi^{\star}$ and the mother wavelet $\psi^{\star}$ are RTI. For the father wavelet $\phi^{\star}(t)=$ $\operatorname{sech}\left(\frac{t}{\sigma}\right)$ to be RTI, I require $\sigma$ such that

$$
\int_{-\infty}^{\infty} \operatorname{sech}\left(\frac{t}{\sigma}\right) e^{-t} d t<\infty
$$

Manipulating the integrand of (4.1.4), I obtain

$$
\operatorname{sech}\left(\frac{t}{\sigma}\right) e^{-t}=2 \exp \left[t\left(\frac{1}{\sigma}-1\right)\right]\left(1+\exp \left[\frac{2 t}{\sigma}\right]\right)^{-1} .
$$

To avoid the integrand becoming infinitely large as $t \rightarrow-\infty$, I require $0<$ $\sigma<1$. Thus, $\phi^{\star}(t)$ is RTI when $0<\sigma<1$.

Similarly for the mother wavelet $\psi^{\star}(t)=\operatorname{sech}\left(\frac{t}{\sigma}\right)\left[1-\frac{t}{\sigma} \tanh \left(\frac{t}{\sigma}\right)\right]$ to be RTI, I require $\sigma$ such that

$$
\int_{-\infty}^{\infty} \operatorname{sech}\left(\frac{t}{\sigma}\right)\left[1-\frac{t}{\sigma} \tanh \left(\frac{t}{\sigma}\right)\right] e^{-t} d t<\infty
$$

Again this constraint is fulfilled when $0<\sigma<1$. Consequently, the basis elements in (4.1.1) are only RTI when $0<\sigma<1$.

If I choose a primary subspace for which $0<\sigma<1$, I have satisfied the physical constraint of finite viscosity. In particular, if $\sigma=\frac{1}{n}(n \in \mathbb{N}, \quad n>1)$, then $2 n-1$ moments exist.

In fitting the data available, it may be necessary to seek a subspace of the Riesz basis $V_{0}$ which is RTI for $\sigma>1$. The simplest of these subspaces are of the form of sech triplets

$$
\operatorname{sech}\left(\frac{t}{\sigma}\right)+A\left[\operatorname{sech}\left(\frac{t+b}{\sigma}\right)+\operatorname{sech}\left(\frac{t-b}{\sigma}\right)\right], \quad b \in \mathbb{R}
$$

I now derive the value of $A$ such that these sech triplets are RTI, i.e.

$$
\int_{-\infty}^{\infty}\left(\operatorname{sech}\left(\frac{t}{\sigma}\right)+A\left[\operatorname{sech}\left(\frac{t+b}{\sigma}\right)+\operatorname{sech}\left(\frac{t-b}{\sigma}\right)\right]\right) e^{-t} d t<\infty .
$$


The integrand decays exponentially as $t \rightarrow \infty$ for all $\sigma>0$. The same is not true, however, as $t \rightarrow-\infty$. By adopting the expansion

$$
\begin{aligned}
\operatorname{sech}\left(\frac{t}{\sigma}\right) e^{-t} & =2 \exp \left[\left(\frac{1}{\sigma}-1\right) t\right]\left(1+\exp \left[\frac{2 t}{\sigma}\right]\right)^{-1} \\
& =2\left(\exp \left[\left(\frac{1}{\sigma}-1\right) t\right]-\exp \left[\left(\frac{3}{\sigma}-1\right) t\right]+\ldots\right)
\end{aligned}
$$

the integrand can be expressed as

$$
\begin{aligned}
& 2\left[1+2 A \cosh \left(\frac{b}{\sigma}\right)\right] \exp \left[\left(\frac{1}{\sigma}-1\right) t\right] \\
& -2\left[1+2 A \cosh \left(\frac{3 b}{\sigma}\right)\right] \exp \left[\left(\frac{3}{\sigma}-1\right) t\right] \\
& +2\left[1+2 A \cosh \left(\frac{5 b}{\sigma}\right)\right] \exp \left[\left(\frac{5}{\sigma}-1\right) t\right]+\ldots
\end{aligned}
$$

By choosing

$$
A=-\frac{1}{2} \operatorname{sech}\left(\frac{b}{\sigma}\right)
$$

the first term vanishes and all higher terms are integrable provided $0<\sigma<3$.

I now investigate the positivity of the sech triplet

$$
\operatorname{sech}\left(\frac{t}{\sigma}\right)-\frac{1}{2} \operatorname{sech}\left(\frac{b}{\sigma}\right)\left[\operatorname{sech}\left(\frac{t+b}{\sigma}\right)+\operatorname{sech}\left(\frac{t-b}{\sigma}\right)\right]
$$

Multiplying by the entirely positive function $\cosh \left(\frac{t}{\sigma}\right) \cosh \left(\frac{t+b}{\sigma}\right) \cosh \left(\frac{t-b}{\sigma}\right)$, 
we find that

$$
\begin{aligned}
& \left\{\operatorname{sech}\left(\frac{t}{\sigma}\right)-\frac{1}{2} \operatorname{sech}\left(\frac{b}{\sigma}\right)\left[\operatorname{sech}\left(\frac{t+b}{\sigma}\right)+\operatorname{sech}\left(\frac{t-b}{\sigma}\right)\right]\right\} \\
& \times \cosh \left(\frac{t}{\sigma}\right) \cosh \left(\frac{t+b}{\sigma}\right) \cosh \left(\frac{t-b}{\sigma}\right) \\
= & \cosh \left(\frac{t+b}{\sigma}\right) \cosh \left(\frac{t-b}{\sigma}\right)-\frac{\cosh \left(\frac{t}{\sigma}\right)}{2 \cosh \left(\frac{b}{\sigma}\right)}\left[\cosh \left(\frac{t+b}{\sigma}\right)+\cosh \left(\frac{t-b}{\sigma}\right)\right] \\
= & 2 \cosh \left(\frac{2 t}{\sigma}\right)+2 \cosh \left(\frac{2 b}{\sigma}\right)-\left[\frac{\cosh \left(\frac{2 t+b}{\sigma}\right)+2 \cosh \left(\frac{b}{\sigma}\right)+\cosh \left(\frac{2 t-b}{\sigma}\right)}{\cosh \left(\frac{b}{\sigma}\right)}\right] \\
= & 2 \cosh \left(\frac{2 t}{\sigma}\right)+2 \cosh \left(\frac{2 b}{\sigma}\right)-\left[2+2 \cosh \left(\frac{2 t}{\sigma}\right)\right] \\
= & 2\left[\cosh \left(\frac{2 b}{\sigma}\right)-1\right] \\
& \geq 0 \quad \forall b \in \mathbb{R}, b>0 .
\end{aligned}
$$

The constraint $0<\sigma<3$ guarantees both the real-time integrability and positivity of the basis function (4.1.12).

It is also possible to find a subspace of $W_{j}(j \leq 0)$ which is RTI for $1 \leq \sigma<3$, but it would be unlikely that mother wavelets would be required in the representation with such low resolution $(\sigma \geq 1)$.

I therefore construct the following approximations for $h$ for $0<\sigma<1$ and $1 \leq \sigma<3$ respectively

$$
\begin{aligned}
h(t) \approx & \sum_{k} b_{k} \phi_{0, k}^{\star}(t)+\sum_{j>0} \sum_{j} a_{j, k} \psi_{j, k}^{\star}(t), \quad j, k \in \mathbf{Z}, \sigma_{0} \in \mathbf{R}, \\
h(t) \approx & \sum_{k} d_{k}\left[\phi_{0,0}^{\star}(t)-\frac{1}{2} \operatorname{sech}\left(\frac{b}{\sigma}\right)\left\{\phi_{0, k}^{\star}(t-b)+\phi_{0, k}^{\star}(t+b)\right\}\right] \\
& +\sum_{\substack{j \\
j>0}} \sum_{k} c_{j, k} \psi_{j, k}^{\star}(t), \quad j, k \in \mathbf{Z}, b, \sigma_{0} \in \mathbf{R} .
\end{aligned}
$$




\subsection{Model Development for $G^{\prime}$ and $G^{\prime \prime}$}

To determine the set of coefficients in the approximation of $h$ I develop new Riesz bases to fit the storage and loss moduli data. I convolute the estimates for $h$ from (4.1.14) and (4.1.15) with the respective kernels of equations (2.1.8) and (2.1.9) to subsequently form models for $g_{1}(x)$ and $g_{2}(x)$. As long as $\sigma$ is rational, all the required convolutions can be calculated analytically via residue calculus. Since $h$ consists of a (mother) wavelet part and a scaling function part, the convolutions required for modeling $g_{1}(x)$ and $g_{2}(x)$ are of the respective forms

$$
\begin{array}{r}
\phi_{j, k}^{\star}(x) \star[1+\tanh (x)], \quad \psi_{j, k}^{\star}(x) \star[1+\tanh (x)], \\
\text { and } \quad\left(\phi_{j, k}^{\star} \star \operatorname{sech}\right)(x), \quad\left(\psi_{j, k}^{\star} \star \operatorname{sech}\right)(x) .
\end{array}
$$

I will consider values of $\sigma=n$ and $\sigma=\frac{1}{n}\left(n \in \mathbb{N}^{+}\right)$for the scaling function convolutions. For values of $\frac{1}{n+1}<\sigma<\frac{1}{n}$ I will construct a homotopic approximation for the models. Adequate resolution of experimental data has never required a value of $\sigma>\frac{1}{2}$ in the mother wavelet part. As a result, I will merely give results for the mother wavelet convolutions for $\sigma=\frac{1}{2 n}\left(n \in \mathbb{N}^{+}\right)$.

\subsubsection{Scaling function convolutions for $G^{\prime}$}

To develop the part of the model for $g_{1}(x)$ emanating from the scaling function part of $h$, I require the following convolutions for different values of $\sigma$ :

$$
\operatorname{sech}\left(\frac{x}{\sigma}\right) \star[1+\tanh (x)]=\sigma \pi+\operatorname{sech}\left(\frac{x}{\sigma}\right) \star \tanh (x)
$$

The convolution

$$
\operatorname{sech}\left(\frac{x}{\sigma}\right) \star \tanh (x)
$$


can be obtained from the convolution theorem for Fourier transforms using the distribution theory developed in section 2.1. As a consequence of (2.1.22), I can write

$$
[\tanh (x)]^{\wedge}(p)=-i \pi \operatorname{cosech}\left(\frac{\pi}{2} p\right)
$$

and

$$
\begin{aligned}
{\left[\operatorname{sech}\left(\frac{x}{\sigma}\right) \star \tanh (x)\right]^{\wedge}(p) } & =\left[\operatorname{sech}\left(\frac{x}{\sigma}\right)\right]^{\wedge}(p)[\tanh (x)]^{\wedge}(p) \\
& =-i \sigma \pi^{2} \operatorname{sech}\left(\frac{\sigma \pi}{2} p\right) \operatorname{cosech}\left(\frac{\pi}{2} p\right)
\end{aligned}
$$

By taking the inverse Fourier transform, I obtain

$$
\operatorname{sech}\left(\frac{x}{\sigma}\right) \star \tanh (x)=\left\{\begin{array}{l}
-\frac{\sigma \pi}{2} i \int_{-\infty}^{\infty} \frac{e^{i p x}}{\cosh \left(\frac{\sigma \pi}{2} p\right) \sinh \left(\frac{\pi}{2} p\right)} d p \quad \text { if } x \neq 0 \\
0 \quad \text { if } x=0
\end{array}\right.
$$

The pole at $p=0$ is integrable. I shall now calculate this integral for different values of $\sigma$. Let

$$
f(z)=\frac{e^{i x z}}{\cosh \left(\frac{\sigma \pi}{2} z\right) \sinh \left(\frac{\pi}{2} z\right)}
$$

$f(z)$ has poles when

$$
\text { (i) } \begin{aligned}
& \cosh \left(\frac{\sigma \pi}{2} z\right)=0 \\
\Rightarrow & z=\frac{2 m-1}{\sigma} i, \quad m \in \mathbb{N}^{+} \\
\text {(ii) } \quad & \sinh \left(\frac{\pi}{2} z\right)=0 \\
\Rightarrow & z=2 m i, \quad m \in \mathbb{N} .
\end{aligned}
$$

Case (i): $\quad \sigma=\frac{1}{n}, \quad n=2 m, \quad m \in \mathbb{N}^{+}$

I use the following indented contour (Fig 4.1). $f(z)$ has $n$ simple poles 


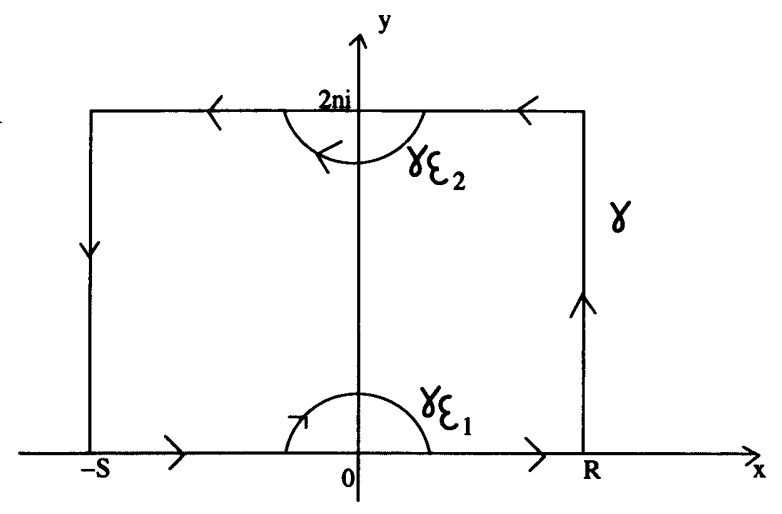

Figure 4.1: Contour $\gamma$

at $z=2 m i\left(m \in \mathbb{N} \backslash\left\{\frac{n}{2}\right\}, m \leq n\right)$, and one pole of order 2 at $z=n i$. Let $f(z)=\frac{g(z)}{h(z)}$, where

$$
\begin{aligned}
& g(z)=\frac{e^{i x z}}{\cosh \left(\frac{\pi}{2 n} z\right)} \\
& h(z)=\sinh \left(\frac{\pi}{2} z\right)
\end{aligned}
$$

Then the residue at the simple poles of $f(z)$ is calculated as follows:

$$
\begin{aligned}
\operatorname{Res}\{f(z) ; 2 m i\} & =\frac{g(2 m i)}{h^{\prime}(2 m i)} \\
& =\frac{2}{\pi} \frac{e^{-2 m x}}{\cosh \left(\frac{m}{n} \pi i\right) \cosh (m \pi i)} \\
& =\frac{2}{\pi}(-1)^{m} \frac{e^{-2 m x}}{\cos \left(\frac{m}{n} \pi\right)} .
\end{aligned}
$$

Since there is a double pole at $z=n i$, I consider a Laurent expansion of $f(z)$ at $z=n i$. The Taylor expansion of $e^{i x z}$ at $z=n i$ is

$$
e^{-n x}\left[1+i x(z-n i)-\frac{1}{2} x^{2}(z-n i)^{2}+\ldots\right]
$$


The Taylor expansion of $\cosh \left(\frac{\pi}{2 n} z\right)$ at $z=n i$ is

$$
\frac{\pi}{2 n} i(z-n i)\left[1+\frac{\pi^{2}}{24 n^{2}}(z-n i)^{2}+\ldots\right] .
$$

The Taylor expansion of $\sinh \left(\frac{\pi}{2} z\right)$ at $z=n i$ is

$$
(-1)^{\frac{n}{2}} \frac{\pi}{2}(z-n i)\left[1+\frac{\pi^{2}}{24}(z-n i)^{2}+\ldots\right] \text {. }
$$

The Laurent expansion of $f(z)$ at $z=n i$ is therefore

$$
\begin{aligned}
& \frac{4 n}{\pi^{2}}(-1)^{\frac{n}{2}+1} i \frac{e^{-n x}[1+i x(z-n i)+\ldots]}{(z-n i)^{2}[1+\ldots]} \\
= & \frac{4 n}{\pi^{2}}(-1)^{\frac{n}{2}+1} i \frac{e^{-n x}[1+i x(z-n i)+\ldots]}{(z-n i)^{2}} .
\end{aligned}
$$

The residue of $f(z)$ at the double pole $z=n i$ is equal to the coefficient of $(z-n i)^{-1}$ in the Laurent expansion. Consequently,

$$
\operatorname{Res}(f, n i)=\frac{4 n}{\pi^{2}}(-1)^{\frac{n}{2}} x e^{-n x} .
$$

Note that

$$
\begin{aligned}
& \lim _{\epsilon_{1} \rightarrow 0} \int_{\gamma_{\epsilon_{1}}} f(z) d z=i \pi \operatorname{Res}\{f(z) ; 0\}=2 i \\
& \lim _{\epsilon_{2} \rightarrow 0} \int_{\gamma_{\epsilon_{2}}} f(z) d z=i \pi \operatorname{Res}\{f(z) ; 2 n i\}=-2 i e^{-2 n x} .
\end{aligned}
$$

By using Cauchy's residue theorem and also by using the same method as shown in Section 2.3 for evaluating the contour integral, as $R, S \rightarrow \infty$ independently and $\epsilon_{1}, \epsilon_{2} \rightarrow 0$, I obtain

$$
\begin{aligned}
& \int_{-\infty}^{\infty} \frac{e^{i p x}}{\cosh \left(\frac{\pi}{2 n} p\right) \sinh \left(\frac{\pi}{2} p\right)} d p-\int_{-\infty}^{\infty} \frac{e^{i x(p+2 n i)}}{\cosh \left(\frac{\pi}{2 n}(p+2 n i)\right) \sinh \left(\frac{\pi}{2}(p+2 n i)\right)} d p \\
= & 2 \pi i \sum_{m=0}^{m=n} \operatorname{Res}\{f(z) ; 2 m i\} .
\end{aligned}
$$


Since $n$ is even, I can simplify the above equation by using the fact that

$$
\begin{aligned}
\cosh \left(\frac{\pi}{2 n}(p+2 n i)\right) & =-\cosh \left(\frac{\pi}{2 n} p\right) \\
\sinh \left(\frac{\pi}{2}(p+2 n i)\right) & =\sinh \left(\frac{\pi}{2} p\right)
\end{aligned}
$$

I consequently obtain

$$
\begin{aligned}
& {\left[1+e^{-2 n x}\right] \int_{-\infty}^{\infty} \frac{e^{i p x}}{\cosh \left(\frac{\pi}{2 n} p\right) \sinh \left(\frac{\pi}{2} p\right)} d p } \\
&= 2 \pi i\left[\frac{1}{\pi}-\frac{2}{\pi} \frac{e^{-2 x}}{\cos \left(\frac{\pi}{n}\right)}+\ldots+\frac{2}{\pi}(-1)^{\frac{n}{2}-1} \frac{e^{-(n-2) x}}{\cos \left(\frac{n-2}{2 n} \pi\right)}+\frac{2}{\pi}(-1)^{\frac{n}{2}+1} \frac{e^{-(n+2) x}}{\cos \left(\frac{n+2}{2 n} \pi\right)}\right. \\
&\left.+\ldots-\frac{1}{\pi} e^{-2 n x}+\frac{4 n}{\pi^{2}}(-1)^{\frac{n}{2}} x e^{-n x}\right] \\
&= 2 i\left[1-e^{-2 n x}-\frac{2}{\cos \left(\frac{\pi}{n}\right)}\left(e^{-2 x}-e^{-(2 n-2) x}\right)+\ldots\right. \\
&\left.+\frac{2(-1)^{\frac{n}{2}+1}}{\cos \left(\frac{n-2}{2 n} \pi\right)}\left(e^{-(n-2) x}-e^{-(n+2) x}\right)+\frac{4 n}{\pi}(-1)^{\frac{n}{2}} x e^{-n x}\right] \\
& \therefore \int_{-\infty}^{\infty} \frac{e^{i p x}}{\cosh \left(\frac{\pi}{2 n} p\right) \sinh \left(\frac{\pi}{2} p\right)} d p=i \operatorname{sech}(n x)\left[e^{n x}-e^{-n x}-\frac{2}{\cos \left(\frac{\pi}{n}\right)}\left(e^{(n-2) x}-e^{-(n-2) x}\right)\right. \\
&\left.+\ldots+\frac{2(-1)^{\frac{n}{2}+1}}{\cos \left(\frac{n-2}{2 n} \pi\right)}\left(e^{2 x}-e^{-2 x}\right)+\frac{4 n}{\pi}(-1)^{\frac{n}{2}} x\right] \\
&= 2 i \operatorname{sech}(n x)\left[\sinh (n x)-\frac{2}{\cos \left(\frac{\pi}{n}\right)} \sinh [(n-2) x]+\ldots+\frac{2(-1)^{\frac{n}{2}+1}}{\cos \left(\frac{n-2}{2 n} \pi\right)} \sinh (2 x)\right. \\
&\left.+\frac{2 n}{\pi}(-1)^{\frac{n}{2}} x\right] \\
&= 2 i \operatorname{sech}(n x)\left[(-1)^{\frac{n}{2}} \frac{2 n}{\pi} x+\sinh (n x)+\sum_{j=1}^{j} \frac{2(-1)^{j}}{\cos \left(\frac{j}{n} \pi\right)} \sinh [(n-2 j) x]\right] \\
& {[}
\end{aligned}
$$


Using equations (4.2.3) and (4.2.7), I finally obtain

$$
\begin{aligned}
& \operatorname{sech}(n x) \star[1+\tanh (x)]=\frac{\pi}{n}(1+\operatorname{sech}(n x) \\
& \left.\left[(-1)^{\frac{n}{2}} \frac{2 n}{\pi} x+\sinh (n x)+\sum_{\substack{k=1 \\
n>2}}^{k=\frac{n-2}{2}} \frac{2(-1)^{k}}{\cos \left(\frac{k}{n} \pi\right)} \sinh [(n-2 k) x]\right]\right) .
\end{aligned}
$$

The results for $n=2(j=1)$ and $n=4(j=2)$ are displayed in Table 4.1. Also, the result for $n=2$ is plotted in Fig. 4.3(a) (in red).

Case (ii): $\quad \sigma=\frac{1}{r}, \quad r=2 m+1, \quad m \in \mathbb{N}$

The integral I now have to calculate is

$$
\int_{-\infty}^{\infty} \frac{e^{i p x}}{\cosh \left(\frac{\pi}{2 r} p\right) \sinh \left(\frac{\pi}{2} p\right)} d p \quad(x \neq 0) .
$$

For this case I use the same indented contour as shown in Fig. 4.1, except for the height of the contour is now $2 r i$. Let

$$
f(z)=\frac{e^{i z x}}{\cosh \left(\frac{\pi}{2 r} z\right) \sinh \left(\frac{\pi}{2} z\right)}
$$

$f(z)$ has $r+1$ simple poles at $z=2 m i(m \in \mathbb{N}, m \leq r)$ which have the same residue as shown in (4.2.13) ( $n$ exchanged with $r$ ), and one simple pole at $z=r i$. Let $f(z)=\frac{j(z)}{k(z)}$, where

$$
\begin{aligned}
& j(z)=\frac{e^{i x z}}{\sinh \left(\frac{\pi}{2} z\right)}, \\
& k(z)=\cosh \left(\frac{\pi}{2 r} z\right) .
\end{aligned}
$$

Then

$$
\begin{aligned}
\operatorname{Res}\{f(z) ; r i\} & =\frac{j(r i)}{k^{\prime}(r i)} \\
& =\frac{2 r}{\pi} \frac{e^{-r x}}{\sinh \left(\frac{r}{2} \pi i\right) \sinh \left(\frac{\pi}{2} i\right)} \\
& =(-1)^{\frac{r+1}{2}} \frac{2 r}{\pi} e^{-r x}
\end{aligned}
$$


Note that

$$
\begin{aligned}
& \lim _{\epsilon_{1} \rightarrow 0} \int_{\gamma_{\epsilon_{1}}} f(z) d z=i \pi \operatorname{Res}\{f(z) ; 0\}=2 i \\
& \lim _{\epsilon_{2} \rightarrow 0} \int_{\gamma_{\epsilon_{2}}} f(z) d z=i \pi \operatorname{Res}\{f(z) ; 2 r i\}=2 i e^{-2 r x}
\end{aligned}
$$

I again use Cauchy's residue theorem to show that, as $R, S \rightarrow \infty$ independently and $\epsilon_{1}, \epsilon_{2} \rightarrow 0$, I obtain

$$
\begin{aligned}
& \int_{-\infty}^{\infty} \frac{e^{i p x}}{\cosh \left(\frac{\pi}{2 r} p\right) \sinh \left(\frac{\pi}{2} p\right)} d p-\int_{-\infty}^{\infty} \frac{e^{i x(p+2 r i)}}{\cosh \left(\frac{\pi}{2 r}(p+2 r i)\right) \sinh \left(\frac{\pi}{2}(p+2 r i)\right)} d p \\
= & {\left[1-e^{-2 r x}\right] \int_{-\infty}^{\infty} \frac{e^{i p x}}{\cosh \left(\frac{\pi}{2 r} p\right) \sinh \left(\frac{\pi}{2} p\right)} d p } \\
= & 2 \pi i\left[\frac{1}{\pi}-\frac{2}{\pi} \frac{e^{-2 x}}{\cos \left(\frac{\pi}{r}\right)}+\ldots+\frac{2}{\pi}(-1)^{\frac{r-1}{2}} \frac{e^{-(r-1) x}}{\cos \left(\frac{r-1}{2 r} \pi\right)}+\frac{2}{\pi}(-1)^{\frac{r+1}{2}} \frac{e^{-(r+1) x}}{\cos \left(\frac{r+1}{2 r} \pi\right)}\right. \\
& \left.+\ldots+\frac{2}{\pi} \frac{e^{-(2 r-2) x}}{\cos \left(\frac{r-1}{r} \pi\right)}+\frac{1}{\pi} e^{-2 r x}+(-1)^{\frac{r+1}{2}} \frac{2 r}{\pi} e^{-r x}\right] \\
= & 2 i\left[1+e^{-2 r x}-\frac{2}{\cos \left(\frac{\pi}{r}\right)}\left(e^{-2 x}+e^{-(2 r-2) x}\right)+\ldots+\frac{2(-1)^{\frac{r-1}{2}}}{\cos \left(\frac{r-1}{2 r} \pi\right)}\left(e^{-(r-1) x}+e^{-(r+1) x}\right)\right. \\
& \left.+2(-1)^{\frac{r+1}{2}} r e^{-r x}\right] \\
\therefore & \int_{-\infty}^{\infty} \frac{e^{i p x}}{\cosh \left(\frac{\pi}{2 r} p\right) \sinh \left(\frac{\pi}{2} p\right)} d p=2 i \operatorname{cosech}(r x)\left[\cosh (r x)-\frac{2}{\cos \left(\frac{\pi}{r}\right)} \cosh [(r-2) x]+\ldots\right. \\
& \left.+\frac{2(-1)^{\frac{r-1}{2}}}{\cos \left(\frac{r-1}{2 r} \pi\right)} \cosh (x)+(-1)^{\frac{r+1}{2}} r\right]
\end{aligned}
$$


Using equations (4.2.3) and (4.2.7), I obtain

$$
\begin{aligned}
& \operatorname{sech}(r x) \star[1+\tanh (x)]=\frac{\pi}{r}\left(1+\operatorname{cosech}(r x)\left[(-1)^{\frac{r+1}{2}} r+\cosh (r x)\right.\right. \\
& \left.\left.+\sum_{\substack{k=1 \\
r>1}}^{k=\frac{r-1}{2}} \frac{2(-1)^{k}}{\cos \left(\frac{k}{r} \pi\right)} \cosh [(r-2 k) x]\right]\right)
\end{aligned}
$$

The result for $r=1(j=0)$ is given in Table 4.1.

Case (iii): $\quad \sigma=m, \quad m \in \mathbb{N}^{+} \backslash\{1\}$.

In this thesis the largest integer value of $\sigma>1$ that I have needed to fit $G^{\prime}$ and $G^{\prime \prime}$ data is $\sigma=2$. The subsequent scaling function convolution for $G^{\prime}$ when $\sigma=2$ is

$$
\operatorname{sech}\left(\frac{x}{2}\right) \star \tanh (x)=-i \pi \int_{-\infty}^{\infty} \frac{e^{i p x}}{\cosh (\pi p) \sinh \left(\frac{\pi}{2} p\right)} d p
$$

Let

$$
f(z)=\frac{e^{i z x}}{\cosh (\pi z) \sinh \left(\frac{\pi}{2} z\right)}
$$

I again use the contour shown in Fig. 4.1, but this time the height of the contour is $2 i$. $f(z)$ has two simple poles within the contour at $z=\frac{1}{2} i$ and $z=\frac{3}{2} i$, with residues

$$
\begin{aligned}
& \operatorname{Res}\left\{f(z) ; \frac{1}{2} i\right\}=-\frac{\sqrt{2}}{\pi} e^{-\frac{x}{2}} \\
& \operatorname{Res}\left\{f(z) ; \frac{3}{2} i\right\}=\frac{\sqrt{2}}{\pi} e^{-\frac{3}{2} x}
\end{aligned}
$$

respectively. There are also two isolated simple poles at $z=0$ and $z=2 i$, which have the following residues:

$$
\begin{aligned}
\operatorname{Res}\{f(z) ; 0\} & =\frac{2}{\pi} \\
\operatorname{Res}\{f(z) ; 2 i\} & =-\frac{2}{\pi} e^{-2 x}
\end{aligned}
$$


Also,

$$
\begin{aligned}
& \lim _{\epsilon_{1} \rightarrow 0} \int_{\gamma_{\epsilon_{1}}} f(z) d z=i \pi \operatorname{Res}\{f(z) ; 0\}=2 i, \\
& \lim _{\epsilon_{2} \rightarrow 0} \int_{\gamma_{\epsilon_{2}}} f(z) d z=i \pi \operatorname{Res}\{f(z) ; 2 i\}=-2 i e^{-2 x} .
\end{aligned}
$$

I again use Cauchy's residue theorem to show that, as $R, S \rightarrow \infty$ independently and $\epsilon_{1}, \epsilon_{2} \rightarrow 0$, I have

$$
\begin{aligned}
& \int_{-\infty}^{\infty} \frac{e^{i p x}}{\cosh (\pi p) \sinh \left(\frac{\pi}{2} p\right)} d p-\int_{-\infty}^{\infty} \frac{e^{i x(p+2 i)}}{\cosh (\pi(p+2 i)) \sinh \left(\frac{\pi}{2}(p+2 i)\right)} d p \\
= & {\left[1+e^{-2 x}\right] \int_{-\infty}^{\infty} \frac{e^{i p x}}{\cosh (\pi p) \sinh \left(\frac{\pi}{2} p\right)} d p } \\
= & 2 i\left[1-e^{-2 x}+\sqrt{2}\left(e^{-\frac{3}{2} x}-e^{-\frac{x}{2}}\right)\right] \\
\therefore & \int_{-\infty}^{\infty} \frac{e^{i p x}}{\cosh (\pi p) \sinh \left(\frac{\pi}{2} p\right)} d p=2 i \operatorname{sech}(x)\left[\sinh (x)-\sqrt{2} \sinh \left(\frac{x}{2}\right)\right] \\
= & 2 i\left[\tanh (x)-\sqrt{2} \operatorname{sech}(x) \sinh \left(\frac{x}{2}\right)\right] .
\end{aligned}
$$

Consequently, using equations (4.2.3) and (4.2.34), I obtain

$$
\operatorname{sech}\left(\frac{x}{2}\right) \star[1+\tanh (x)]=2 \pi\left[1+\tanh (x)-\sqrt{2} \operatorname{sech}(x) \sinh \left(\frac{x}{2}\right)\right] .
$$

The result for $m=2(j=-1)$ is given in Table 4.1 .

\subsubsection{Scaling function convolutions for $G^{\prime \prime}$}

To develop the part of the model for $g_{2}(x)$ emanating from the scaling function part of $h$, I require the following convolutions for different values of $\sigma$ :

$$
\operatorname{sech}\left(\frac{x}{\sigma}\right) \star \operatorname{sech}(x)
$$




\begin{tabular}{|c|c|}
\hline$j$ & $\operatorname{sech}\left(2^{j} x\right) \star[1+\tanh (x)]$ \\
\hline-1 & $2 \pi\left[1+\tanh (x)-\sqrt{2} \operatorname{sech}(x) \sinh \left(\frac{x}{2}\right)\right]$ \\
\hline 0 & $\pi[1+\operatorname{coth}(x)-\operatorname{cosech}(x)] \quad(x \neq 0)$ \\
& $\pi \quad(x=0)$ \\
\hline 1 & $\frac{1}{2} \pi[1+\tanh (2 x)]-2 x \operatorname{sech}(2 x)$ \\
\hline 2 & $\frac{\pi}{4}\left(1+\operatorname{sech}(4 x)\left[\sinh (4 x)-2 \sqrt{(2)} \sinh (2 x)+\frac{8}{\pi} x\right]\right)$ \\
\hline
\end{tabular}

Table 4.1: Scaling function convolutions for fitting $G^{\prime}$ data, with $\sigma=2^{-j}$.

By taking the Fourier transform of (4.2.44), and using the convolution theorem,

$$
\left[\operatorname{sech}\left(\frac{x}{\sigma}\right) \star \operatorname{sech}(x)\right]^{\wedge}(p)=\sigma \pi^{2} \operatorname{sech}\left(\frac{\sigma \pi}{2} p\right) \operatorname{sech}\left(\frac{\pi}{2} p\right)
$$

By taking the inverse Fourier transform, I obtain

$$
\operatorname{sech}\left(\frac{x}{\sigma}\right) \star \operatorname{sech}(x)=\frac{\sigma \pi}{2} \int_{-\infty}^{\infty} \frac{e^{i p x}}{\cosh \left(\frac{\sigma \pi}{2} p\right) \cosh \left(\frac{\pi}{2} p\right)} d p .
$$

I shall now calculate this integral for different values of $\sigma$.

Case (i): $\quad \sigma=\frac{1}{n}, \quad n=2 m, \quad m \in \mathbb{N}^{+}$

I use the contour displayed below in Fig 4.2. Let

$$
f(z)=\frac{e^{i z x}}{\cosh \left(\frac{\pi}{2 n} z\right) \cosh \left(\frac{\pi}{2} z\right)} .
$$

Within the contour $\gamma, f(z)$ has $n$ simple poles at $z=(2 m+1) i(m \in \mathbb{N}$, $m \leq n-1)$ and a simple pole at $z=n i$. Let $f(z)=\frac{g(z)}{h(z)}$, where

$$
\begin{aligned}
g(z) & =\frac{e^{i z x}}{\cosh \left(\frac{\pi}{2 n} z\right)} \\
h(z) & =\cosh \left(\frac{\pi}{2} z\right)
\end{aligned}
$$




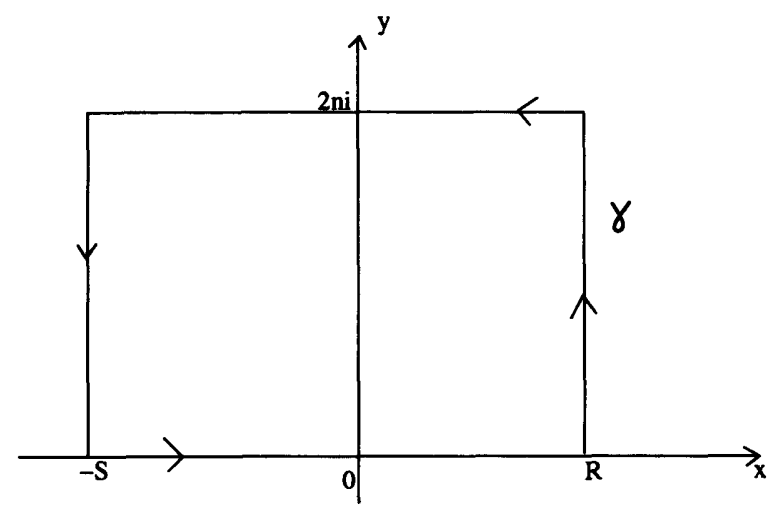

Figure 4.2: Contour $\gamma$

Then

$$
\begin{aligned}
\operatorname{Res}\{f(z) ;(2 m+1) i\} & =\frac{g[(2 m+1) i]}{h^{\prime}[(2 m+1) i]} \\
& =\frac{2}{\pi} \frac{e^{-(2 m+1) x}}{\cosh \left(\frac{2 m+1}{2 n} \pi i\right) \sinh \left(\frac{2 m+1}{2} \pi i\right)} \\
& =\frac{2}{\pi}(-1)^{m+1} i \frac{e^{-(2 m+1) x}}{\cos \left(\frac{2 m+1}{2 n} \pi\right)} .
\end{aligned}
$$

Now let $f(z)=\frac{j(z)}{k(z)}$, where

$$
\begin{aligned}
& j(z)=\frac{e^{i z x}}{\cosh \left(\frac{\pi}{2} z\right)}, \\
& k(z)=\cosh \left(\frac{\pi}{2 n} z\right) .
\end{aligned}
$$

Then

$$
\begin{aligned}
\operatorname{Res}\{f(z) ; n i\} & =\frac{j[n i]}{k^{\prime}[n i]} \\
& =\frac{2 n}{\pi} \frac{e^{-n x}}{\cosh \left(\frac{\pi}{2} n i\right) \sinh \left(\frac{\pi}{2} i\right)} \\
& =\frac{2 n}{\pi}(-1)^{\frac{n}{2}+1} i e^{-n x}
\end{aligned}
$$


By Cauchy's residue theorem, as $R, S \rightarrow \infty$ independently, I obtain

$$
\begin{aligned}
& \int_{-\infty}^{\infty} \frac{e^{i p x}}{\cosh \left(\frac{\pi}{2 n} p\right) \cosh \left(\frac{\pi}{2} p\right)} d p-\int_{-\infty}^{\infty} \frac{e^{i x(p+2 n i)}}{\cosh \left(\frac{\pi}{2 n}(p+2 n i)\right) \cosh \left(\frac{\pi}{2}(p+2 n i)\right)} d p \\
= & {\left[1+e^{-2 n x}\right] \int_{-\infty}^{\infty} \frac{e^{i p x}}{\cosh \left(\frac{\pi}{2 n} p\right) \cosh \left(\frac{\pi}{2} p\right)} d p } \\
= & 2 \pi i\left[\frac{2}{\pi} i \sum_{k=0}^{n-1}(-1)^{k+1} \frac{e^{-(2 k+1) x}}{\cos \left(\frac{2 k+1}{2 n} \pi\right)}+\frac{2 n}{\pi}(-1)^{\frac{n}{2}+1} i e^{-n x}\right] \\
\therefore & \int_{-\infty}^{\infty} \frac{e^{i p x}}{\cosh \left(\frac{\pi}{2 n} p\right) \cosh \left(\frac{\pi}{2} p\right)} d p=2 \operatorname{sech}(n x)\left[\sum_{k=0}^{n-1}(-1)^{k} \frac{e^{[n-(2 k+1)] x}}{\cos \left(\frac{2 k+1}{2 n} \pi\right)}+(-1)^{\frac{n}{2}} n\right] \\
= & 2 \operatorname{sech}(n x)\left[2 \sum_{\substack{k=0 \\
n \geq 2}}^{k=\frac{n-2}{2}} \frac{(-1)^{k}}{\cos \left(\frac{2 k+1}{2 n} \pi\right)} \cosh ([n-(2 k+1)] x)+(-1)^{\frac{n}{2}} n\right] .
\end{aligned}
$$

Consequently, using equation (4.2.46), I obtain

$$
\begin{aligned}
& \operatorname{sech}(n x) \star \operatorname{sech}(x)=\frac{\pi}{n} \operatorname{sech}(n x)\left((-1)^{\frac{n}{2}} n\right. \\
& \left.+\sum_{\substack{k=0 \\
n \geq 2}}^{k=\frac{n-2}{2}} \frac{2(-1)^{k}}{\cos \left(\frac{2 k+1}{2 n} \pi\right)} \cosh [(n-(2 k+1)) x]\right)
\end{aligned}
$$

The results for $n=2(j=1)$ and $n=4(j=2)$ are displayed in Table 4.2. Also, the result for $n=2$ is plotted in Fig. 4.3(a) (in blue).

Case (ii): $\quad \sigma=\frac{1}{r}, \quad r=2 m+1, \quad m \in \mathbb{N}$

The integral I now have to calculate is

$$
\int_{-\infty}^{\infty} \frac{e^{i p x}}{\cosh \left(\frac{\pi}{2 r} p\right) \cosh \left(\frac{\pi}{2} p\right)} d p
$$

For this case I use the same contour as shown in Fig. 4.2, except for the height of the contour which is now $2 r i$. Let

$$
f(z)=\frac{e^{i z x}}{\cosh \left(\frac{\pi}{2 r} z\right) \cosh \left(\frac{\pi}{2} z\right)} .
$$


$f(z)$ has $r-1$ simple poles at $z=(2 m+1) i\left(m \in \mathbb{N} \backslash\left\{\frac{r-1}{2}\right\}, m \leq r-1\right)$ which have the same residue as shown in (4.2.50) ( $n$ exchanged with $r$ ), and one double pole at $z=r i$. To calculate the residue of the pole at $z=r i$, I construct a Laurent expansion of $f(z)$ at $z=r i$. By exchanging $n$ for $r$ in (4.2.14) and (4.2.15), I obtain respective Taylor expansions for $e^{i z x}$ and $\cosh \left(\frac{\pi}{2 r} z\right)$, at $z=r i$. The Taylor expansion of $\cosh \left(\frac{\pi}{2} z\right)$ at $z=r i$ is given by

$$
(-1)^{\frac{r-1}{2}} \frac{\pi}{2} i(z-r i)\left[1+\frac{1}{6}\left(\frac{\pi}{2}\right)^{2}(z-r i)^{2}+\ldots\right]
$$

Hence it can be shown that the Laurent expansion of $f(z)$ at $z=r i$ is

$$
=-\frac{4 r}{\pi^{2}}(-1)^{\frac{r-1}{2}} \frac{e^{-r x}[1+i x(z-r i)-\ldots]}{(z-r i)^{2}} .
$$

The residue of $f(z)$ at the double pole $z=r i$ is equal to the coefficient of $(z-r i)^{-1}$ in the Laurent expansion. Consequently,

$$
\operatorname{Res}(f, r i)=-\frac{4 r}{\pi^{2}}(-1)^{\frac{r-1}{2}} i x e^{-r x} \text {. }
$$

By Cauchy's residue theorem, as $R, S \rightarrow \infty$ independently, I obtain

$$
\begin{aligned}
& \int_{-\infty}^{\infty} \frac{e^{i p x}}{\cosh \left(\frac{\pi}{2 r} p\right) \cosh \left(\frac{\pi}{2} p\right)} d p-\int_{-\infty}^{\infty} \frac{e^{i x(p+2 r i)}}{\cosh \left(\frac{\pi}{2 r}(p+2 r i)\right) \cosh \left(\frac{\pi}{2}(p+2 r i)\right)} d p \\
= & {\left[1-e^{-2 r x}\right] \int_{-\infty}^{\infty} \frac{e^{i p x}}{\cosh \left(\frac{\pi}{2 r} p\right) \cosh \left(\frac{\pi}{2} p\right)} d p }
\end{aligned}
$$




$$
\begin{aligned}
& =2 \pi i\left[-\frac{4 r}{\pi^{2}}(-1)^{\frac{r-1}{2}} i x e^{-r x}+\frac{2}{\pi} i \sum_{\substack{k=0 \\
k \neq \frac{r-1}{2}}}^{r-1}(-1)^{k+1} \frac{e^{-(2 k+1) x}}{\cos \left(\frac{2 k+1}{2 r} \pi\right)}\right] \\
& \therefore \int_{-\infty}^{\infty} \frac{e^{i p x}}{\cosh \left(\frac{\pi}{2 r} p\right) \cosh \left(\frac{\pi}{2} p\right)} d p \\
& =2 \operatorname{cosech}(r x)\left[\frac{2 r}{\pi}(-1)^{\frac{r-1}{2}} x+\sum_{\substack{k=0 \\
k \neq \frac{r-1}{2}}}^{r-1}(-1)^{k+1} \frac{e^{(r-(2 k+1)] x}}{\cos \left(\frac{2 k+1}{2 r} \pi\right)}\right] \\
& =4 \operatorname{cosech}(r x)\left[\frac{r}{\pi}(-1)^{\frac{r-1}{2}} x+\sum_{\substack{k=0 \\
k=0}}^{r=3}(-1)^{k+1} \frac{\sinh ([r-(2 k+1)] x)}{\cos \left(\frac{2 k+1}{2 r} \pi\right)}\right] .
\end{aligned}
$$

Consequently, using equation (4.2.46), I obtain

$$
\begin{gathered}
\operatorname{sech}(r x) \star \operatorname{sech}(x)=\frac{2 \pi}{r} \operatorname{cosech}(r x)\left((-1)^{\frac{r-1}{2}} \frac{r}{\pi} x\right. \\
\left.+\sum_{\substack{k=0 \\
r \geq 3}}^{k=\frac{r-3}{2}} \frac{(-1)^{k}}{\cos \left(\frac{2 k+1}{2 r} \pi\right)} \sinh [(r-(2 k+1)) x]\right) .
\end{gathered}
$$

The result for $r=1(j=0)$ is given in Table 4.2.

Case (iii): $\quad \sigma=m, \quad m \in \mathbb{N}^{+} \backslash\{1\}$.

When $\sigma$ takes integer values, I derive the following simple equation for $\operatorname{sech}\left(\frac{x}{m}\right) \star \operatorname{sech}(x)$ in terms of the results for $\operatorname{sech}(m x) \star \operatorname{sech}(x)$ given in cases (i) and (ii).

$$
\begin{array}{rlr}
\operatorname{sech}\left(\frac{x}{m}\right) \star \operatorname{sech}(x) & =\int_{-\infty}^{\infty} \operatorname{sech}\left(x-\frac{s}{m}\right) \operatorname{sech}(s) d s \\
& =m \int_{-\infty}^{\infty} \operatorname{sech}(x-t) \operatorname{sech}(m t) d t \quad(m t=s) \\
& =m \operatorname{sech}(m x) \star \operatorname{sech}(x) .
\end{array}
$$

The result for $\sigma=2(j=-1)$ is given in Table 4.2 below. 


\begin{tabular}{|c|c|}
\hline$j$ & $\operatorname{sech}\left(2^{j} x\right) \star \operatorname{sech}(x)$ \\
\hline-1 & $2 \pi \operatorname{sech}(x)\left[\sqrt{2} \cosh \left(\frac{x}{2}\right)-1\right]$ \\
\hline 0 & $2 x \operatorname{cosech}(x)(x \neq 0)$ \\
& $2 \quad(x=0)$ \\
\hline 1 & $\pi \operatorname{sech}(2 x)[\sqrt{2} \cosh (x)-1]$ \\
\hline 2 & $\pi \operatorname{sech}(4 x)\left[A^{-\frac{1}{2}} \cosh (3 x)-B^{-\frac{1}{2}} \cosh (x)+1\right]$ \\
\hline
\end{tabular}

Table 4.2: Scaling function convolutions for fitting $G^{\prime \prime}$ data, where $A=2+\sqrt{2}$ and $B=2-\sqrt{2}$, and $\sigma=2^{-j}$.

\subsubsection{Wavelet convolutions for $G^{\prime}$}

To develop the part of the model for $g_{1}(x)$ emanating from the mother wavelet part of $h$, I require the following convolutions for different values of $\sigma:$

$$
\begin{aligned}
{[1+\tanh (x)] \star \operatorname{sech}\left(\frac{x}{\sigma}\right)\left[1-\frac{x}{\sigma} \tanh \left(\frac{x}{\sigma}\right)\right] } & =\tanh (x) \star \operatorname{sech}\left(\frac{x}{\sigma}\right)\left[1-\frac{x}{\sigma} \tanh \left(\frac{x}{\sigma}\right)\right] \\
& =\tanh (x) \star \frac{d}{d x}\left[x \operatorname{sech}\left(\frac{x}{\sigma}\right)\right] \\
& =\frac{d}{d x}[\tanh (x)] \star\left[x \operatorname{sech}\left(\frac{x}{\sigma}\right)\right] \\
& =\operatorname{sech}^{2}(x) \star\left[x \operatorname{sech}\left(\frac{x}{\sigma}\right)\right],
\end{aligned}
$$

where I first used the fact that the mother wavelet $\operatorname{sech}\left(\frac{x}{\sigma}\right)\left[1-\frac{x}{\sigma} \tanh \left(\frac{x}{\sigma}\right)\right]$ has zero area, and then used the derivative property of convolutions. Using 
equation (2.1.22) and properties of the Fourier transform, I find that

$$
\begin{aligned}
\left(\operatorname{sech}^{2}(x) \star\left[x \operatorname{sech}\left(\frac{x}{\sigma}\right)\right]\right)^{\wedge}(p) & =\left[\operatorname{sech}^{2}\right]^{\wedge}(p)\left[x \operatorname{sech}\left(\frac{x}{\sigma}\right)\right]^{\wedge}(p) \\
& =\left(\pi p \operatorname{cosech}\left(\frac{\pi}{2} p\right)\right)\left(i \sigma \pi \frac{d}{d p}\left[\operatorname{sech}\left(\frac{\sigma \pi}{2} p\right)\right]\right) \\
& =-\frac{\sigma^{2} \pi^{3}}{2} i p \operatorname{cosech}\left(\frac{\pi}{2} p\right) \operatorname{sech}\left(\frac{\sigma \pi}{2} p\right) \tanh \left(\frac{\sigma \pi}{2} p\right) \\
& =-\frac{\sigma^{2} \pi^{3}}{2} i p \frac{\sinh \left(\frac{\sigma \pi}{2} p\right)}{\sinh \left(\frac{\pi}{2} p\right) \cosh ^{2}\left(\frac{\sigma \pi}{2} p\right)} .
\end{aligned}
$$

By taking the inverse Fourier transform of (4.2.65), I obtain

$$
\begin{aligned}
\tanh (x) \star \frac{d}{d x}\left[x \operatorname{sech}\left(\frac{x}{\sigma}\right)\right] & =-\left(\frac{\sigma \pi}{2}\right)^{2} i \int_{-\infty}^{\infty} \frac{p \sinh \left(\frac{\sigma \pi}{2} p\right) e^{i p x}}{\sinh \left(\frac{\pi}{2} p\right) \cosh ^{2}\left(\frac{\sigma \pi}{2} p\right)} d p \\
& =-\left(\frac{\sigma \pi}{2}\right)^{2} \frac{d}{d x} \int_{-\infty}^{\infty} \frac{\sinh \left(\frac{\sigma \pi}{2} p\right) e^{i p x}}{\sinh \left(\frac{\pi}{2} p\right) \cosh ^{2}\left(\frac{\sigma \pi}{2} p\right)} d p
\end{aligned}
$$

I consider values of $\sigma=\frac{1}{n}$, where $n=2 m, m \in \mathbb{N}^{+}$, when calculating the above convolution. Let

$$
f(z)=\frac{\sinh \left(\frac{\sigma \pi}{2} z\right) e^{i z x}}{\sinh \left(\frac{\pi}{2} z\right) \cosh ^{2}\left(\frac{\sigma \pi}{2} z\right)} .
$$

Using (4.2.9) and (4.2.10), it is clear that $f(z)$ has simple poles at $z=2 m i$ $(m \in \mathbb{N})$ and double poles when $z=(2 m-1) n i\left(m \in \mathbb{N}^{+}\right)$. When using the same contour as shown in Fig. 4.1, $f(z)$ has $n$ simple poles at $z=2 m i$ $\left(m \in \mathbb{N} \backslash\left\{\frac{n}{2}\right\}, m \leq n\right)$, and one pole of order 3 at $z=n i$. Let $f(z)=\frac{g(z)}{h(z)}$, where

$$
\begin{aligned}
& g(z)=\frac{\sinh \left(\frac{\pi}{2 n} z\right) e^{i x z}}{\cosh ^{2}\left(\frac{\pi}{2 n} z\right)} \\
& h(z)=\sinh \left(\frac{\pi}{2} z\right)
\end{aligned}
$$


Then the residue at the simple poles of $f(z)$ is calculated as follows:

$$
\begin{aligned}
\operatorname{Res}\{f(z) ; 2 m i\} & =\frac{g(2 m i)}{h^{\prime}(2 m i)} \\
& =\frac{2}{\pi} \frac{\sinh \left(\frac{m}{n} \pi i\right) e^{-2 m x}}{\cosh ^{2}\left(\frac{m}{n} \pi i\right) \cosh (m \pi i)} \\
& =\frac{2}{\pi}(-1)^{m} i \tan \left(\frac{m}{n} \pi\right) \sec \left(\frac{m}{n} \pi\right) e^{-2 m x}
\end{aligned}
$$

From (4.2.70) note that

$$
\begin{aligned}
& \operatorname{Res}\{f(z) ; 0\}=\operatorname{Res}\{f(z) ; 2 n i\}=0 \\
& \therefore \lim _{\epsilon_{1} \rightarrow 0} \int_{\gamma_{\epsilon_{1}}} f(z) d z=\lim _{\epsilon_{2} \rightarrow 0} \int_{\gamma_{\epsilon_{2}}} f(z) d z=0 .
\end{aligned}
$$

Since there is a triple pole at $z=n i$, I consider a Laurent expansion of $f(z)$ at $z=n i$. The Taylor expansions of $e^{i x z}$ and $\sinh \left(\frac{\pi}{2} z\right)$, at $z=n i$, is given in (4.2.14) and (4.2.16), respectively. Also, from (4.2.15), it can be seen that the Taylor expansion of $\cosh ^{2}\left(\frac{\pi}{2 n} z\right)$ at $z=n i$ is given by

$$
-\left(\frac{\pi}{2 n}\right)^{2}(z-n i)^{2}\left[1+\frac{1}{3}\left(\frac{\pi}{2 n}\right)^{2}(z-n i)^{2}+\ldots\right]
$$

Furthermore, the Taylor expansion of $\sinh \left(\frac{\pi}{2 n} z\right)$ at $z=n i$ is given by

$$
i\left[1+\frac{1}{2}\left(\frac{\pi}{2 n}\right)^{2}(z-n i)^{2}+\ldots\right]
$$


Consequently, the Laurent expansion of $f(z)$ at $z=n i$ is given by

$$
\begin{aligned}
& \frac{8 n^{2}}{\pi^{3}}(-1)^{\frac{n}{2}+1} i \frac{e^{-n x}\left[1+i x(z-n i)+\frac{1}{2}\left[\left(\frac{\pi}{2 n}\right)^{2}-x^{2}\right](z-n i)^{2}+\ldots\right]}{(z-n i)^{3}\left[1+\left\{\frac{1}{6}\left(\frac{\pi}{2}\right)^{2}+\frac{1}{3}\left(\frac{\pi}{2 n}\right)^{2}\right\}(z-n i)^{2}+\ldots\right]} \\
= & \frac{8 n^{2}}{\pi^{3}(z-n i)^{3}}(-1)^{\frac{n}{2}+1} i e^{-n x}\left[1+i x(z-n i)+\frac{1}{2}\left[\left(\frac{\pi}{2 n}\right)^{2}-x^{2}\right](z-n i)^{2}+\ldots\right] \\
& \times\left[1-\frac{1}{6}\left(\frac{\pi}{2}\right)^{2}\left(1+\frac{2}{n^{2}}\right)^{2}(z-n i)^{2}-\ldots\right] \\
= & \frac{8 n^{2}(-1)^{\frac{n}{2}+1}}{\pi^{3}(z-n i)^{3}} i e^{-n x}\left[1+i x(z-n i)+\left\{\frac{1}{2}\left[\left(\frac{\pi}{2 n}\right)^{2}-x^{2}\right]\right.\right. \\
& \left.\left.-\frac{1}{6}\left(\frac{\pi}{2}\right)^{2}\left(1+\frac{2}{n^{2}}\right)^{2}\right\}(z-n i)^{2}+\ldots\right] .
\end{aligned}
$$

The residue of $f(z)$ at the triple pole $z=n i$ is equal to the coefficient of $(z-n i)^{-1}$ in the Laurent expansion. Consequently,

$$
\operatorname{Res}(f ; n i)=\frac{4 n^{2}(-1)^{\frac{n}{2}+1}}{3 \pi^{3}} i\left[\left(\frac{\pi}{2 n}\right)^{2}-\left(\frac{\pi}{2}\right)^{2}-3 x^{2}\right] e^{-n x}
$$

Hence, by Cauchy's residue theorem with $R, S \rightarrow \infty$ independently, I obtain

$$
\begin{aligned}
& \int_{-\infty}^{\infty} \frac{\sinh \left(\frac{\pi}{2 n} p\right) e^{i x p}}{\sinh \left(\frac{\pi}{2} p\right) \cosh ^{2}\left(\frac{\pi}{2 n} p\right)} d p-\int_{-\infty}^{\infty} \frac{\sinh \left[\frac{\pi}{2 n}(p+2 n i)\right] e^{i x(p+2 n i)}}{\sinh \left[\frac{\pi}{2}(p+2 n i)\right] \cosh ^{2}\left[\frac{\pi}{2 n}(p+2 n i)\right]} d p \\
= & {\left[1+e^{-2 n x}\right] \int_{-\infty}^{\infty} \frac{\sinh \left(\frac{\pi}{2 n} p\right) e^{i x p}}{\sinh \left(\frac{\pi}{2} p\right) \cosh ^{2}\left(\frac{\pi}{2 n} p\right)} d p }
\end{aligned}
$$




$$
\begin{aligned}
= & 2 \pi i\left\{\frac{2}{\pi} i \sum_{\substack{k=0 \\
k \neq \frac{n}{2}}}^{k=n}(-1)^{k} \tan \left(\frac{k}{n} \pi\right) \sec \left(\frac{k}{n} \pi\right) e^{-2 k x}\right. \\
& \left.+\frac{4 n^{2}(-1)^{\frac{n}{2}+1}}{3 \pi^{3}} i\left[\left(\frac{\pi}{2 n}\right)^{2}-\left(\frac{\pi}{2}\right)^{2}-3 x^{2}\right] e^{-n x}\right\} . \\
\therefore & \int_{-\infty}^{\infty} \frac{\sinh \left(\frac{\pi}{2 n} p\right) e^{i x p}}{\sinh \left(\frac{\pi}{2} p\right) \cosh ^{2}\left(\frac{\pi}{2 n} p\right)} d p=2 \operatorname{sech}(n x)\left\{\frac{2 n^{2}(-1)^{\frac{n}{2}}}{3 \pi^{2}}\left[\left(\frac{\pi}{2 n}\right)^{2}-\left(\frac{\pi}{2}\right)^{2}-3 x^{2}\right]\right. \\
& \left.-\sum_{\substack{k=0 \\
k=\frac{n}{2}}}^{k=n}(-1)^{k} \tan \left(\frac{k}{n} \pi\right) \sec \left(\frac{k}{n} \pi\right) e^{(n-2 k) x}\right\} \\
= & 4 \operatorname{sech}(n x)\left\{\frac{n^{2}(-1)^{\frac{n}{2}}}{3 \pi^{2}}\left[\left(\frac{\pi}{2 n}\right)^{2}-\left(\frac{\pi}{2}\right)^{2}-3 x^{2}\right]\right. \\
& \left.-\sum_{k=0}^{k=\frac{n}{2}-1}(-1)^{k} \tan \left(\frac{k}{n} \pi\right) \sec \left(\frac{k}{n} \pi\right)^{2} \cosh [(n-2 k) x]\right\} .
\end{aligned}
$$

Using (4.2.66),

$$
\begin{aligned}
& \tanh (x) \star \frac{d}{d x}[x \operatorname{sech}(n x)]=-\frac{\pi^{2}}{n^{2}} \frac{d}{d x}\left[\operatorname { s e c h } ( n x ) \left\{\frac{n^{2}(-1)^{\frac{n}{2}}}{3 \pi^{2}}\left[\left(\frac{\pi}{2 n}\right)^{2}-\left(\frac{\pi}{2}\right)^{2}-3 x^{2}\right]\right.\right. \\
& \left.\left.-\sum_{k=0}^{k=\frac{n}{2}-1}(-1)^{k} \tan \left(\frac{k}{n} \pi\right) \sec \left(\frac{k}{n} \pi\right) \cosh [(n-2 k) x]\right\}\right] \\
& =\left(\frac{\pi}{n}\right)^{2} \operatorname{sech}(n x)\left\{\sum_{k=0}^{k=\frac{n}{2}-1}(-1)^{k}(n-2 k) \tan \left(\frac{k}{n} \pi\right) \sec \left(\frac{k}{n} \pi\right) \sinh [(n-2 k) x]\right. \\
& -\frac{(-1)^{\frac{n}{2}+1} 2 n^{2}}{\pi^{2}} x^{2}+n \tanh (n x)\left[\frac{n^{2}(-1)^{\frac{n}{2}}}{3 \pi^{2}}\left[\left(\frac{\pi}{2 n}\right)^{2}-\left(\frac{\pi}{2}\right)^{2}-3 x^{2}\right]\right. \\
& \left.-\sum_{k=0}^{k=\frac{n}{2}-1}(-1)^{k} \tan \left(\frac{k}{n} \pi\right) \sec \left(\frac{k}{n} \pi\right) \cosh [(n-2 k) x]\right\} .
\end{aligned}
$$

I list the following table of results for $n=2(j=1)$ and $n=4(j=2)$. The result for $n=2$ is plotted in Fig. 4.3(b) (in red). 


\begin{tabular}{|c|c|}
\hline$j$ & $\left(\operatorname{sech}\left(2^{j} x\right)\left[1-2^{j} x \tanh \left(2^{j} x\right)\right]\right) \star[1+\tanh (x)]$ \\
\hline 1 & $\frac{1}{8} \operatorname{sech}(2 x)\left[\left(\pi^{2}+16 x^{2}\right) \tanh (2 x)-16 x\right]$ \\
\hline 2 & $-\frac{\pi^{2}}{64} \operatorname{sech}(4 x)\left(8 \sqrt{2} \sinh (2 x)-\frac{128}{\pi^{2}} x\right.$ \\
& $\left.-4 \tanh (4 x)\left[4 \sqrt{2} \cosh (2 x)-\frac{64}{\pi^{2}} x^{2}-5\right]\right)$ \\
\hline
\end{tabular}

Table 4.3: Wavelet convolutions for $G^{\prime}$ data, where $\sigma=2^{-j}$.

\subsubsection{Wavelet convolutions for $G^{\prime \prime}$}

To develop the part of the model for $g_{2}(x)$ emanating from the mother wavelet part of $h$, I require the following convolutions for different values of $\sigma:$

$$
\operatorname{sech}(x) \star \frac{d}{d x}\left[x \operatorname{sech}\left(\frac{x}{\sigma}\right)\right] .
$$

Using (2.1.13) and (4.2.65), it can be shown that the Fourier transform of (4.2.79) is given by

$$
\begin{aligned}
{\left[\operatorname{sech}(x) \star \frac{d}{d x}\left[x \operatorname{sech}\left(\frac{x}{\sigma}\right)\right]\right]^{\wedge}(p) } & =[\operatorname{sech}(x)]^{\wedge}(p) \star\left[\frac{d}{d x}\left[x \operatorname{sech}\left(\frac{x}{\sigma}\right)\right]\right]^{\wedge}(p) \\
& =\frac{\sigma^{2} \pi^{3}}{2} p \operatorname{sech}\left(\frac{\pi}{2} p\right) \operatorname{sech}\left(\frac{\sigma \pi}{2} p\right) \tanh \left(\frac{\sigma \pi}{2} p\right) \\
& =\frac{\sigma^{2} \pi^{3}}{2} p \frac{\sinh \left(\frac{\sigma \pi}{2} p\right)}{\cosh \left(\frac{\pi}{2} p\right) \cosh ^{2}\left(\frac{\sigma \pi}{2} p\right)} .
\end{aligned}
$$

By taking the inverse Fourier transform, I obtain

$$
\begin{aligned}
\operatorname{sech}(x) \star \frac{d}{d x}\left[x \operatorname{sech}\left(\frac{x}{\sigma}\right)\right] & =\left(\frac{\sigma \pi}{2}\right)^{2} \int_{-\infty}^{\infty} \frac{p \sinh \left(\frac{\sigma \pi}{2} p\right) e^{i p x}}{\cosh \left(\frac{\pi}{2} p\right) \cosh ^{2}\left(\frac{\sigma \pi}{2} p\right)} d p \\
& =-\left(\frac{\sigma \pi}{2}\right)^{2} i \frac{d}{d x} \int_{-\infty}^{\infty} \frac{\sinh \left(\frac{\sigma \pi}{2} p\right) e^{i p x}}{\cosh \left(\frac{\pi}{2} p\right) \cosh ^{2}\left(\frac{\sigma \pi}{2} p\right)} d p
\end{aligned}
$$


For the same reason as in section 4.2.3, I will only consider values values of $\sigma=\frac{1}{n}$, where $n=2 m, m \in \mathbb{N}^{+}$. Let

$$
f(z)=\frac{\sinh \left(\frac{\pi}{2 n} z\right) e^{i z x}}{\cosh \left(\frac{\pi}{2} z\right) \cosh ^{2}\left(\frac{\pi}{2 n} z\right)}
$$

Using the same contour as displayed in Fig. $4.2, f(z)$ has $n$ simple poles at $z=(2 m+1) i(m \in \mathbb{N}, m \leq n-1)$ and a double pole at $z=n i$. I calculate the residue at the $n$ simple poles as follows. Let $f(z)=\frac{g(z)}{h(z)}$, where

$$
\begin{aligned}
& g(z)=\frac{\sinh \left(\frac{\pi}{2 n} z\right) e^{i z x}}{\cosh ^{2}\left(\frac{\pi}{2 n} z\right)} \\
& h(z)=\cosh \left(\frac{\pi}{2} z\right)
\end{aligned}
$$

Then

$$
\begin{aligned}
\operatorname{Res}\{f(z) ;(2 m+1) i\} & =\frac{g[(2 m+1) i]}{h^{\prime}[(2 m+1) i]} \\
& =\frac{2}{\pi} \frac{\sinh \left(\frac{2 m+1}{2 n} \pi i\right) e^{-(2 m+1) x}}{\sinh \left(\frac{2 m+1}{2} \pi i\right) \cosh ^{2}\left(\frac{2 m+1}{2 n} \pi i\right)} \\
& =\frac{2}{\pi}(-1)^{m} \frac{\sin \left(\frac{2 m+1}{2 n} \pi\right) e^{-(2 m+1) x}}{\cos ^{2}\left(\frac{2 m+1}{2 n} \pi\right)} \\
& =\frac{2}{\pi}(-1)^{m} \sec \left(\frac{2 m+1}{2 n} \pi\right) \tan \left(\frac{2 m+1}{2 n} \pi\right) e^{-(2 m+1) x}
\end{aligned}
$$

Since there is a double pole at $z=n i$, I consider a Laurent expansion of $f(z)$ at $z=n i$. The Taylor expansions of $e^{i x z}, \cosh ^{2}\left(\frac{\pi}{2 n} z\right)$ and $\sinh \left(\frac{\pi}{2 n} z\right)$, at $z=n i$, are displayed in (4.2.14), (4.2.73) and (4.2.74), respectively. The Taylor expansion of $\cosh \left(\frac{\pi}{2} z\right)$ at $z=n i$ is given by

$$
(-1)^{\frac{n}{2}}\left[1+\frac{\pi^{2}}{8}(z-n i)^{2}+\ldots\right]
$$


Hence it can be shown that the Laurent expansion of $f(z)$ at $z=n i$ is given by

$$
(-1)^{\frac{n}{2}+1}\left(\frac{2 n}{\pi}\right)^{2} i \frac{e^{-n x}[1+i x(z-n i)+\ldots]}{(z-n i)^{2}}
$$

The residue of $f(z)$ at the double pole $z=n i$ is equal to the coefficient of $(z-n i)^{-1}$ in the Laurent expansion. Consequently,

$$
\operatorname{Res}(f ; n i)=(-1)^{\frac{n}{2}}\left(\frac{2 n}{\pi}\right)^{2} x e^{-n x} .
$$

Hence, by Cauchy's residue theorem with $R, S \rightarrow \infty$ independently, I obtain

$$
\begin{aligned}
& \int_{-\infty}^{\infty} \frac{\sinh \left(\frac{\pi}{2 n} p\right) e^{i p x}}{\cosh \left(\frac{\pi}{2} p\right) \cosh ^{2}\left(\frac{\pi}{2 n} p\right)} d p-\int_{-\infty}^{\infty} \frac{\sinh \left(\frac{\pi}{2 n}(p+2 n i)\right) e^{i x(p+2 n i)}}{\cosh \left(\frac{\pi}{2}(p+2 n i)\right) \cosh ^{2}\left(\frac{\pi}{2 n}(p+2 n i)\right)} d p \\
= & {\left[1+e^{-2 n x}\right] \int_{-\infty}^{\infty} \frac{\sinh \left(\frac{\pi}{2 n} p\right) e^{i p x}}{\cosh \left(\frac{\pi}{2} p\right) \cosh ^{2}\left(\frac{\pi}{2 n} p\right)} d p } \\
= & 2 \pi i\left[\frac{2}{\pi} \sum_{k=0}^{n-1}(-1)^{k} \sec \left(\frac{2 k+1}{2 n} \pi\right) \tan \left(\frac{2 k+1}{2 n} \pi\right) e^{-(2 k+1) x}+(-1)^{\frac{n}{2}}\left(\frac{2 n}{\pi}\right)^{2} x e^{-n x}\right] \\
\therefore & \int_{-\infty}^{\infty} \frac{\sinh \left(\frac{\pi}{2 n} p\right) e^{i p x}}{\cosh \left(\frac{\pi}{2} p\right) \cosh ^{2}\left(\frac{\pi}{2 n} p\right)} d p=\pi i \operatorname{sech}(n x)\left[(-1)^{\frac{n}{2}}\left(\frac{2 n}{\pi}\right)^{2} x\right. \\
& \left.+\frac{2}{\pi} \sum_{k=0}^{n-1}(-1)^{k} \sec \left(\frac{2 k+1}{2 n} \pi\right) \tan \left(\frac{2 k+1}{2 n} \pi\right) e^{[n-(2 k+1)] x}\right] \\
= & \pi i \operatorname{sech}(n x)\left[\frac{4}{\pi} \sum_{k=0}^{n-1}(-1)^{k} \sec \left(\frac{2 k+1}{2 n} \pi\right) \tan \left(\frac{2 k+1}{2 n} \pi\right) \sinh ([n-(2 k+1)] x)\right. \\
& \left.+(-1)^{\frac{n}{2}}\left(\frac{2 n}{\pi}\right)^{2} x\right] .
\end{aligned}
$$


Using (4.2.81),

$$
\begin{aligned}
& \operatorname{sech}(x) \star \frac{d}{d x}[x \operatorname{sech}(n x)]=\left(\frac{\pi}{2 n}\right)^{2} \frac{d}{d x}\left\{\operatorname { s e c h } ( n x ) \left[(-1)^{\frac{n}{2}} \frac{(2 n)^{2}}{\pi} x\right.\right. \\
& \left.\left.+4 \sum_{k=0}^{n-1}(-1)^{k} \sec \left(\frac{2 k+1}{2 n} \pi\right) \tan \left(\frac{2 k+1}{2 n} \pi\right) \sinh ([n-(2 k+1)] x)\right]\right\} \\
& =\quad\left(\frac{\pi}{2 n}\right)^{2} \operatorname{sech}(n x)\left\{(-1)^{\frac{n}{2}} \frac{(2 n)^{2}}{\pi}\right. \\
& +4 \sum_{k=0}^{n-1}(-1)^{k}[n-(2 k+1)] \sec \left(\frac{2 k+1}{2 n} \pi\right) \tan \left(\frac{2 k+1}{2 n} \pi\right) \cosh ([n-(2 k+1)] x) \\
& -n \tanh (n x)\left[(-1)^{\frac{n}{2}} \frac{(2 n)^{2}}{\pi} x\right. \\
& \left.\left.+4 \sum_{k=0}^{n-1}(-1)^{k} \sec \left(\frac{2 k+1}{2 n} \pi\right) \tan \left(\frac{2 k+1}{2 n} \pi\right) \sinh ([n-(2 k+1)] x)\right]\right\}
\end{aligned}
$$

I list the following table of results for $n=2(j=1)$ and $n=4(j=2)$. The result for $n=2$ is plotted in Fig. 4.3(b) (in blue).

\begin{tabular}{|l|c|}
\hline$j$ & $\left(\operatorname{sech}\left(2^{j} x\right)\left[1-2^{j} x \tanh \left(2^{j} x\right)\right]\right) \star \operatorname{sech}(x)$ \\
\hline 1 & $\frac{\pi^{2}}{4} \operatorname{sech}(2 x)\left(\sqrt{2} \cosh (x)-\frac{4}{\pi}\right.$ \\
& $\left.-2 \tanh (2 x)\left[\sqrt{2} \sinh (x)-\frac{4}{\pi}(x)\right]\right)$ \\
\hline 2 & $\frac{\pi^{2}}{8} \operatorname{sech}(4 x)\left(\frac{3}{2} B^{\frac{3}{2}} \cosh (3 x)-\frac{1}{2} A^{\frac{3}{2}} \cosh (x)+\frac{8}{\pi}\right.$ \\
& $\left.-4 \tanh (4 x)\left[\frac{1}{2} B^{\frac{3}{2}} \sinh (3 x)-\frac{1}{2} A^{\frac{3}{2}} \sinh (x)+\frac{8}{\pi}(x)\right]\right)$ \\
\hline
\end{tabular}

Table 4.4: Wavelet convolutions for $G^{\prime \prime}$ data, where $A=2+\sqrt{2}$ and $B=$ $2-\sqrt{2}$, and $\sigma=2^{-j}$. 

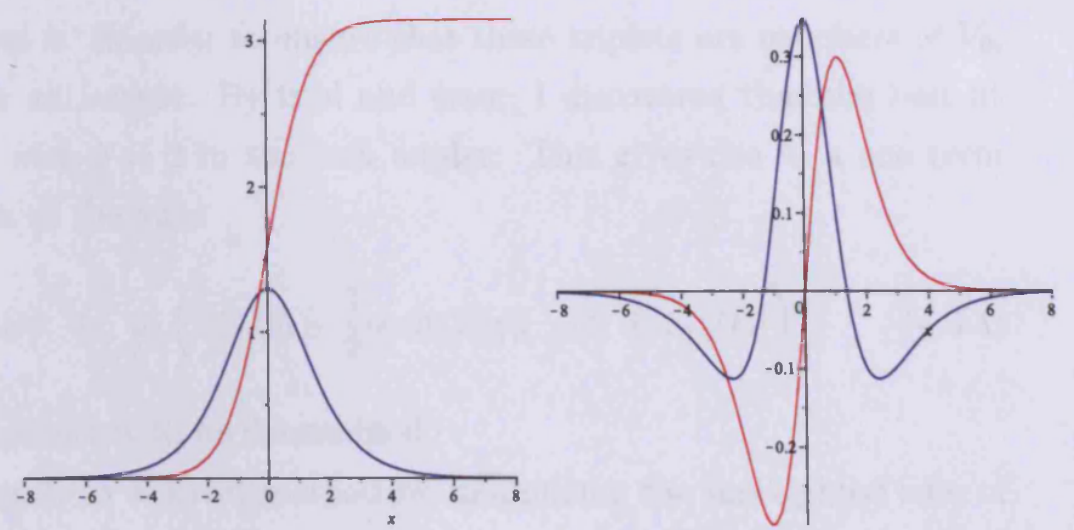

(a) $\phi_{1,0}^{\star}(x) \star(1+\tanh (x))($ red $),\left(\phi_{1,0}^{\star} \star\left(\right.\right.$ b) $\left(\psi_{1,0}^{\star} \star(1+\tanh )\right)(x)($ red $),\left(\psi_{1,0}^{\star} \star\right.$ $\operatorname{sech}(x)$ (blue) $\operatorname{sech})(x)$ (blue)

Figure 4.3: Examples of scaling function convolutions and wavelet convolutions used for fitting $G^{\prime}$ and $G^{\prime \prime}$ data when $\sigma=\frac{1}{2}$.

\subsection{Approximation of the Gaussian}

I will now approximate the Gaussian spectrum as a test case. I use a Gaussian with unit standard deviation, i.e.

$$
h(t)=e^{-\frac{t^{2}}{2}} .
$$

\subsection{1 sech triplet approximation}

At resolution $\sigma=1$ (i.e. $J=0$ ), I have the Riesz basis

$$
V_{0}=\cos _{L^{2}(\mathbb{R})} \operatorname{span}\{\operatorname{sech}(x-k)\}, \quad k \in \mathbb{Z},
$$

with sampling rate $a=1$. Since $\sigma=1$, I require sech triplets of the form

$$
\operatorname{sech}(x)-\frac{1}{2} \operatorname{sech}(b)[\operatorname{sech}(x-b)+\operatorname{sech}(x+b)]
$$


to approximate $h$. In order to ensure that these triplets are members of $V_{0}$, I need $b$ to be an integer. By trial and error, I discovered that the best fit was achieved with $b=2$ in the sech triplet. This gives rise to a one term approximation of the form

$$
h(t) \approx b_{1}\left(\phi_{0,0}^{\star}(t)-\frac{1}{2} \operatorname{sech}(2)\left[\phi_{0,-2}^{\star}(t)+\phi_{0,2}^{\star}(t)\right]\right)
$$

where $b_{1}$ is a constant to be determined.

I determine $b_{1}$ in a direct method by minimizing the unweighted sum of squared residuals

$$
\sum_{j}\left[\Delta_{0}\left(t_{j}\right)\right]^{2}
$$

where

$$
\Delta_{0}(t)=h(t)-b_{1}\left(\phi_{0,0}^{\star}(t)-\frac{1}{2} \operatorname{sech}(2)\left[\phi_{0,-2}^{\star}(t)+\phi_{0,2}^{\star}(t)\right]\right),
$$

evaluated on the discrete point set $t_{j}=0.5 j, \quad j=-16, \ldots, 16$. This gives rise to the approximation, displayed against the actual spectrum, in Fig. 4.4(a), with $b_{1}=1.0889$ and a root mean square (RMS) error of $0.332 \%$. The maximum error of $1.20 \times 10^{-2}$ is shown in Fig. 4.4(b).

However, the correct method to determine $b_{1}$ is through fitting my convoluted models for $g_{1}(x)$ and $g_{2}(x)$ (developed earlier in this chapter) to the storage and loss moduli data for the Gaussian spectrum. I calculated numerically the noise-free storage and loss moduli data for the Gaussian spectrum at the sample points $t_{j}$ given above. The data is plotted with the Gaussian in Fig. 4.5. I simultaneously fit my models for $g_{1}(x)$ and $g_{2}(x)$ to the data, by minimizing the unweighted sum of squared residuals

$$
\sum_{j}\left[\Delta_{1}\left(t_{j}\right)\right]^{2}+\sum_{j}\left[\Delta_{2}\left(t_{j}\right)\right]^{2}
$$


where

$$
\begin{aligned}
& \Delta_{1}\left(t_{j}\right)=G^{\prime}\left(t_{j}\right)-g_{1}\left(t_{j}\right) \\
& \Delta_{2}\left(t_{j}\right)=G^{\prime \prime}\left(t_{j}\right)-g_{2}\left(t_{j}\right)
\end{aligned}
$$

and where

$$
\begin{aligned}
& g_{1}(x)=b_{1}\left(\phi_{0,0}^{\star}(x)-\frac{1}{2} \operatorname{sech}(2)\left[\phi_{0,-2}^{\star}(x)+\phi_{0,2}^{\star}(x)\right]\right) \star[1+\tanh (x)], \\
& g_{2}(x)=b_{1}\left(\phi_{0,0}^{\star}(x)-\frac{1}{2} \operatorname{sech}(2)\left[\phi_{0,-2}^{\star}(x)+\phi_{0,2}^{\star}(x)\right]\right) \star \operatorname{sech}(x) .
\end{aligned}
$$

I obtained a fit to the data of $0.087 \%\left(b_{1}=1.0871\right)$ and a recovered spectrum with a fit to the Gaussian with an RMS error of $0.336 \%$ (Fig. 4.6(a)). The maximum error in the recovered spectrum is $1.09 \times 10^{-2}$ (Fig. $4.6(\mathrm{~b}))$.

\subsubsection{Calderón enriched approximation}

Although I can get a very good approximation for the Gaussian using $V_{0}$ alone, I can increase the accuracy of the approximation further by enriching my basis with elements of the space $W_{1}$, defined in (3.6.19) and (3.6.20), where

$$
\psi_{j, k}^{\star}(x)=2^{\frac{j}{2}} \psi^{\star}\left(2^{j} x-k a\right)
$$

with sampling rate $a=1$ and

$$
\psi^{\star}(x)=\operatorname{sech}(x)[1-x \tanh (x)]
$$

I choose basis functions from $W_{1}$ centred at the point of maximum residual in the sech triplet approximation, illustrated in Fig. 4.6(b). This gives rise 
to an enriched approximation for $h$ of the form

$$
\begin{aligned}
h(t) \approx & b_{1}\left(\phi_{0,0}^{\star}(t)-\frac{1}{2} \operatorname{sech}(2)\left[\phi_{0,-2}^{\star}(t)+\phi_{0,2}^{\star}(t)\right]\right) \\
& +a_{1,2}\left[\psi_{1,-2}^{\star}(t)+\psi_{1,2}^{\star}(t)\right],
\end{aligned}
$$

where $b_{1}$ and $a_{1,2}$ are now the constants to be determined. The form of the approximation is chosen to guarantee symmetry.

I determine $b_{1}$ and $a_{1,2}$ in a direct method, in the same way as explained in the sech triplet approximation. This gives rise to the approximation, displayed against the actual spectrum, in Fig. 4.7(a), with $b_{1}=1.0872$ and $a_{1,2}=0.0122$ and a root mean square (RMS) error of $0.133 \%$. The maximum error of $4.61 \times 10^{-3}$ is shown in Fig. 4.7(b).

I simultaneously fitted the models for $g_{1}(x)$ and $g_{2}(x)$ to the storage and loss moduli data (Fig. 4.8(a)), in the same way as explained in the sech triplet approximation. We obtained a superb fit to the data of $8.47 \times 10^{-3} \%$ $\left(b_{1}=1.0867, a_{1,2}=0.0132\right)$, and a recovered spectrum with an RMS error of $0.136 \%$. The maximum error in the recovered spectrum is $4.77 \times 10^{-3}$ (Fig.4.8(b)).

I can increase the accuracy of our approximation even further by enriching my basis with elements of the space $W_{2}$. I choose basis functions from $W_{2}$ centred at the point of maximum residual in the first enriched approximation, illustrated in Fig. 4.8(b). This gives rise to an enriched approximation for $h$ of the form

$$
\begin{aligned}
h(t) \approx & b_{1}\left(\phi_{0,0}^{\star}(t)-\frac{1}{2} \operatorname{sech}(2)\left[\phi_{0,-2}^{\star}(t)+\phi_{0,2}^{\star}(t)\right]\right) \\
& +a_{1,2}\left[\psi_{1,-2}^{\star}(t)+\psi_{1,2}^{\star}(t)\right] \\
& +a_{2,2}\left[\psi_{2,-2}^{\star}(t)+\psi_{2,2}^{\star}(t)\right]+a_{2,3}\left[\psi_{2,-3}^{\star}(t)+\psi_{2,3}^{\star}(t)\right],
\end{aligned}
$$

where $b_{1}, a_{1,2}, a_{2,2}$ and $a_{2,3}$ are now the constants to be determined. 
In the direct approximation, I have $b_{1}=1.0870, a_{1,2}=0.0114, a_{2,2}=$ 0.0037 and $a_{2,3}=0.0068$, with an RMS error of $0.0142 \%$ (Fig. 4.9(a)) and a maximum error of $2.92 \times 10^{-3}$ (Fig. $4.9(\mathrm{~b})$ ).

I simultaneously fitted the models for $g_{1}(x)$ and $g_{2}(x)$ to the storage and loss moduli data (Fig. 4.10(a)). I obtained a remarkable fit to the data with an RMS error of $6.14 \times 10^{-4} \%\left(b_{1}=1.0867, a_{1,2}=0.0115, a_{2,2}=0.0056\right.$, $\left.a_{2,3}=0.0034\right)$ and a recovered spectrum with an RMS error of $0.0409 \%$. The maximum error in the recovered spectrum is $1.11 \times 10^{-3}$ (Fig. $4.10(\mathrm{~b})$ ).

The coefficients and RMS error values from the three direct approximations of the Gaussian given above are displayed in Table 4.5, whereas those for the three approximations of the Gaussian using the storage and loss moduli data are given in Table 4.6.

\begin{tabular}{|c|c|c|c|}
\hline & sech triplet & $\begin{array}{c}\text { Calderón enrichment } \\
\left(W_{1}\right)\end{array}$ & $\begin{array}{c}\text { Calderón enrichment } \\
\left(W_{1} \text { and } W_{2}\right)\end{array}$ \\
\hline$b_{1}$ & 1.0889 & 1.0872 & 1.0872 \\
\hline$a_{1,2}$ & & 0.0122 & 0.0114 \\
\hline$a_{2,2}$ & & & 0.0037 \\
\hline$a_{2,3}$ & & 0.133 & 0.0068 \\
\hline $\begin{array}{c}\text { Spectrum (\%) } \\
\text { RMS error }\end{array}$ & 0.332 & & 0.0142 \\
\hline Maximum error & $1.20 \times 10^{-2}$ & $4.61 \times 10^{-3}$ & $2.92 \times 10^{-3}$ \\
\hline
\end{tabular}

Table 4.5: Direct approximations of the Gaussian. 


\begin{tabular}{|c|c|c|c|}
\hline & sech triplet & $\begin{array}{c}\text { Calderón enrichment } \\
\left(W_{1}\right)\end{array}$ & $\begin{array}{c}\text { Calderón enrichment } \\
\left(W_{1} \text { and } W_{2}\right)\end{array}$ \\
\hline$b_{1}$ & 1.0871 & 1.0867 & 1.0867 \\
\hline$a_{1,2}$ & & 0.0132 & 0.0115 \\
\hline$a_{2,2}$ & & & 0.0056 \\
\hline$a_{2,3}$ & & & 0.0034 \\
\hline Data RMS error $(\%)$ & 0.0874 & $8.47 \times 10^{-3}$ & $6.14 \times 10^{-4}$ \\
\hline Spectrum RMS error $(\%)$ & 0.336 & 0.136 & 0.0409 \\
\hline Maximum error & $1.09 \times 10^{-2}$ & $4.77 \times 10^{-3}$ & $1.11 \times 10^{-3}$ \\
\hline
\end{tabular}

Table 4.6: Approximations of the Gaussian using the storage and loss moduli data.

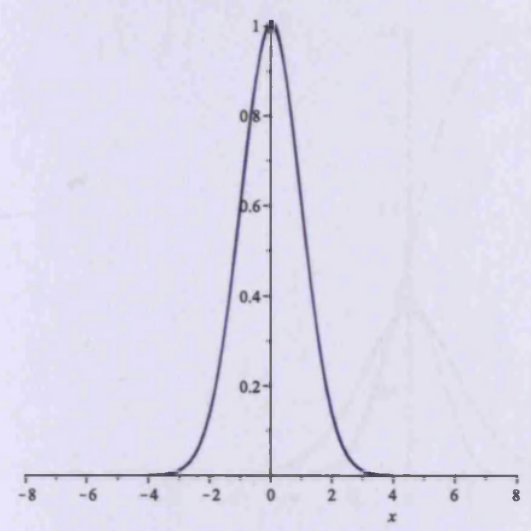

(a) Direct approximation.



(b) Error in the direct approximation. Maximum error $=1.20 \times 10^{-2}$.

Figure 4.4: Direct approximation of the Gaussian spectrum with a sech triplet $\left(b_{1}=1.0889, \sigma=1, b=2, a=1\right.$, RMS error $\left.=0.332 \%\right)$. 


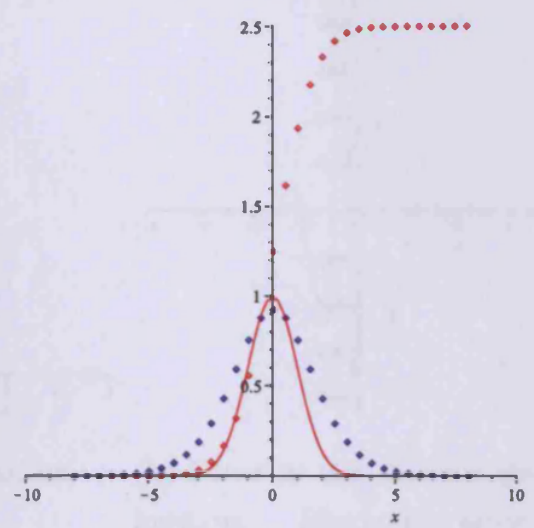

Figure 4.5: $G^{\prime}$ (red) and $G^{\prime \prime}$ (blue) data calculated from the above Gaussian spectrum.
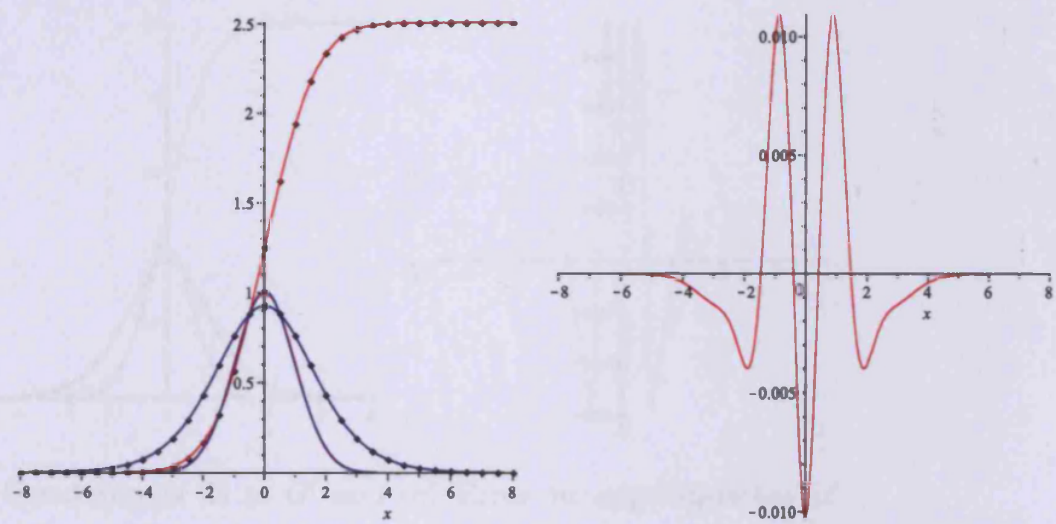

(a) Simultaneous fit to $G^{\prime}$ and $G^{\prime \prime}$ (b) Error in approximation of Gausdata, with resulting spectrum. sian. Maximum error $=1.09 \times 10^{-2}$.

Figure 4.6: Simultaneous fit to $G^{\prime}$ and $G^{\prime \prime}$ data by sech triplet convoluted models. Resulting fit to spectrum with RMS error $=0.336 \%$. 


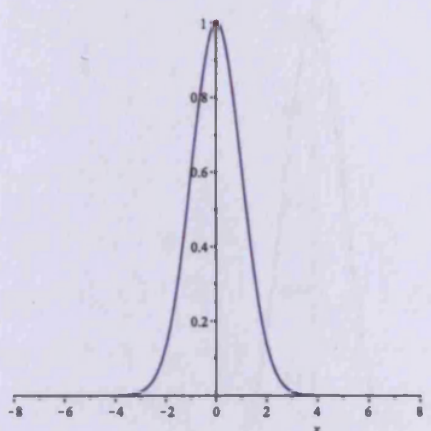

(a) Direct approximation.

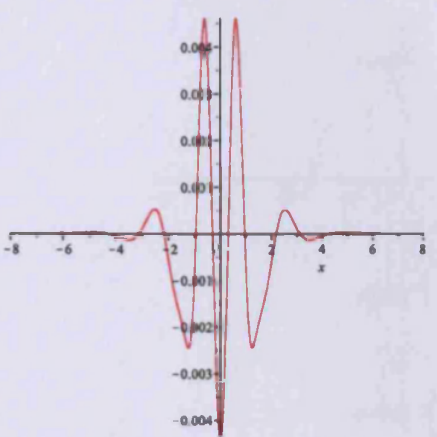

(b) Error in the direct approximation. Maximum error $=$ $4.61 \times 10^{-3}$.

Figure 4.7: Direct approximation of the Gaussian spectrum using Calderón enrichment (one mother wavelet).


(a) Simultaneous fit to $G^{\prime}$ and (b) Error in approximation of $G^{\prime \prime}$ data, with resulting spec- Gaussian. Maximum error = trum. $4.77 \times 10^{-3}$.

Figure 4.8: Simultaneous fit to $G^{\prime}$ and $G^{\prime \prime}$ data by convoluted models, using Calderón enrichment (one mother wavelet). Resulting fit to spectrum with RMS error $=0.136 \%$. 


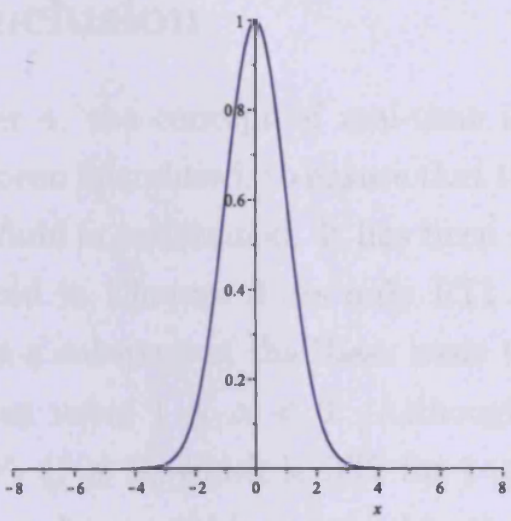

(a) Direct approximation.

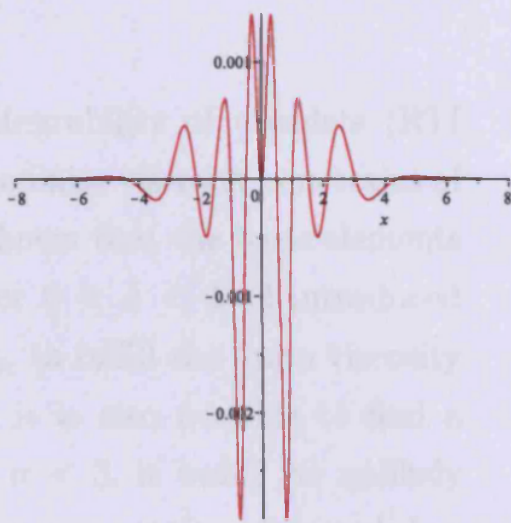

(b) Error in the direct approximation.

Figure 4.9: Direct approximation of the Gaussian spectrum using further Calderón enrichment (three mother wavelets).



(a) Simultaneous fit to $G^{\prime}$ and $G^{\prime \prime}$ data, with resulting spectrum.

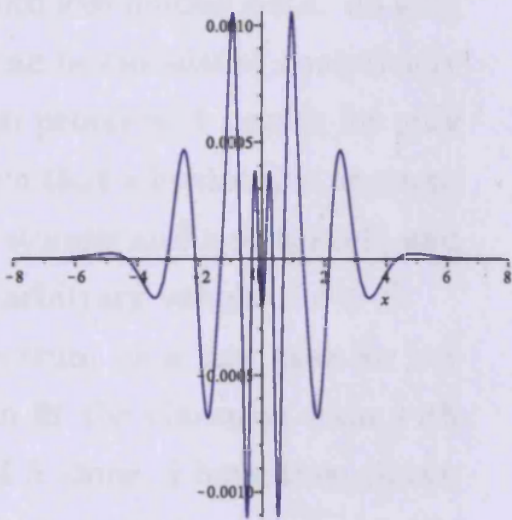

(b) Error in approximation of Gaussian. Maximum error $=1.11 \times 10^{-3}$.

Figure 4.10: Simultaneous fit to $G^{\prime}$ and $G^{\prime \prime}$ data by convoluted models, using Calderón enrichment (three mother wavelets). Resulting fit to spectrum with RMS error $=0.0409 \%$. 


\subsection{Conclusion}

In Chapter 4, the concept of real-time integrability of wavelets (RTI wavelets) has been introduced, to ensure that the finite viscosity constraint of a viscoelastic fluid is maintained. It has been shown that the basis elements for $h$ introduced in Chapter 3 are only RTI for $0<\sigma<1$. I introduced sech triplets as a subspace of the Riesz basis $V_{0}$, to fulfill the finite viscosity constraint when using $1 \leq \sigma<3$. Although it is also possible to find a subspace of $W_{j}(j \leq 0)$ which is RTI for $1 \leq \sigma<3$, it would be unlikely that mother wavelets would be required in the representation with such low resolution $(\sigma>1)$. The above theory has enabled me to give more concise estimates for $h$, with representations for $0<\sigma<1$ and $1 \leq \sigma<3$ given by equations (4.1.14) and (4.1.15), respectively.

The main part of this chapter has been concerned with the development of models for $g_{1}(x)$ and $g_{2}(x)$, to fit the storage and loss moduli data. As long as $\sigma$ is rational, all of the required convolutions can be calculated analytically via residue calculus. In this chapter I have given proofs and results for only selected values of $\sigma$. In Chapter 5 it will be shown that a homotopic approximation can be used to construct models for the storage and loss moduli, and indeed the continuous relaxation spectrum, for arbitrary values of $\sigma>0$.

I have also approximated the Gaussian spectrum as a test case for my models in this chapter. I have shown that I can fit the Gaussian data with good accuracy using the scaling function part of $h$ alone. I have then shown that Calderón enrichment can be introduced to give exceptional accuracy. In this chapter I have given results for synthetic, noise-free data. In the next chapter I will concentrate on real data, and also synthetic data with and without added noise. 


\section{Chapter 5}

\section{Wavelet Regularization}

\subsection{Introduction}

Regularization is required in the numerical treatment of any deconvolution problem with smooth kernel, in order to control the instability inherent in the inversion process. The instability manifests itself in the amplification of any noise in the data. Even very small amounts of noise (e.g. rounding error in the computer representations of real numbers) can lead to very high levels of noise amplification in the solution. In dealing with dynamic data from an oscillatory shear experiment, levels of noise of around 1 or $2 \%$ are not unusual, and the recovery of a relaxation spectrum is not possible without some form of regularization.

I will illustrate the need for, and nature of, regularization by considering the deconvolution problem

$$
\operatorname{sech}(x) \star h(x)=g(x)
$$

where $h(x)$ and $g(x)$ are in $L^{2}(\mathbb{R})$. Let $\nu(x)$ be the noise induced in $h(x)$ by noise $n(x)$ in $g(x)$, i.e. assume

$$
\operatorname{sech}(x) \star[h(x)+\nu(x)]=g(x)+n(x) .
$$


Then

$$
\operatorname{sech}(x) \star \nu(x)=n(x)
$$

and taking Fourier transforms I find that

$$
\widehat{\nu}(p)=\frac{1}{\pi} \cosh \left(\frac{\pi}{2} p\right) \widehat{n}(p) .
$$

The Fourier transform of the noise in $g(x)$ is therefore amplified exponentially by the growth in $\cosh \left(\frac{\pi}{2} p\right)$ as $p \rightarrow \pm \infty$.

This noise amplification is brought under control by applying a filter $z(x)$ with Fourier transform $\widehat{z}(p)$. In the act of regularization, equation (5.1.2) is replaced by its filtered approximation

$$
\operatorname{sech}(x) \star h_{F}(x)=g_{F}(x)
$$

where $g_{F}(x)$ and $h_{F}(x)$ are regularized approximations to $g(x)$ and $h(x)$, respectively, and $g_{F}(x)$ takes the form

$$
g_{F}(x)=z(x) \star[g(x)+n(x)] .
$$

It follows immediately that

$$
\widehat{h_{F}}(p)=\widehat{z}(p)\left[\frac{1}{\pi} \cosh \left(\frac{\pi}{2} p\right)\right][\widehat{g}(p)+\widehat{n}(p)],
$$

and consequently if a regularized approximation in $L^{2}(\mathbb{R})$ is required, the filter must be strong enough to ensure

$$
\widehat{z}(p)\left[\frac{1}{\pi} \cosh \left(\frac{\pi}{2} p\right)\right] \widehat{n}(p) \rightarrow 0 \quad \text { as } \quad p \rightarrow \pm \infty .
$$

In other words the filter $z$ removes the amplified noise in (5.1.3) at high frequencies.

The way in which the filter $z(x)$ is chosen is determined by the method of regularization. Not every regularization method ensures that the recovered 
relaxation spectrum is in $L^{2}(\mathbb{R})$. For example, in Tikhonov regularization, the resulting classes of filter have been described and analysed by Anderssen and Davies (1986 [27]). In Tikhonov's method the Fourier transform of the filter can be written down explicitly in the form

$$
\widehat{z}(p)=\frac{1}{1+\lambda p^{2 n}},
$$

where $\lambda$ denotes a shape parameter, and the integer $n$ controls the rate of decay of the filter. The rate of decay of the Tikhonov filter is, by definition, algebraic, and is not suited to stabilizing the exponential growth in noise observed in (5.1.3). Despite this, Honerkamp [43] and co-workers have attempted to use Tikhonov regularization in recovering the relaxation spectrum. Such an approach is destined to limited success since (5.1.8) can never be satisfied with the choice of filter in (5.1.9).

In their sparse spike deconvolution method, Baumgaertel and Winter [14], create a filter which cannot be written down explicitly. Nevertheless it is easily shown (see below) that the filter implicitly created by Baumgaertel and Winter is not strong enough to satisfy (5.1.8). In their approach, the filter is such that $\widehat{z}(p)\left[\frac{1}{\pi} \cosh \left(\frac{\pi}{2} p\right)\right]$ remains bounded as $p \rightarrow \pm \infty$, which is consistent with the representation of the regularized solution as a sum delta-functions.

In this thesis I mainly represent the relaxation spectrum as a wavelet series in the space $V_{0}$ with scale $\sigma$. I shall show that, as in the case of Baumgaertel and Winter, the filters I introduce through wavelet regularization cannot be written down explicitly, but, in contrast to the method of Baumgaertel and Winter, my filters are strong enough to guarantee that (5.1.8) holds.

Previous work connected with wavelet regularization includes the work by Shen and Walter [68] (who introduce a regularization procedure based on band-limited orthogonal wavelets- the Meyer wavelets) and also the work by 
Antoniadis and Fan [9] (who introduce nonlinear regularized wavelet estimators for estimating nonparametric regression functions when sampling points are not uniformly spaced).

\subsection{Continuous wavelet dictionaries}

Definition 5.1: I define the set of functions

$$
D_{\sigma}=\left\{\operatorname{sech}\left(\frac{t-k \mu}{\sigma}\right): k \in \mathbb{Z}\right\}
$$

to be a dictionary. The dictionary is characterized by its sampling rate $\mu$ and its scale $\sigma$. The sampling rate is the distance between the central positions of adjacent wavelets. Each element of the dictionary is called an atom. The elements of $D_{\sigma}$ are linearly independent, and any $d$ separate atoms in $D_{\sigma}$ span a $d$-dimensional subspace of $V_{0}$.

In this chapter I will focus on approximating the continuous relaxation spectrum $h(t)$ by selecting elements from the dictionary $D_{\sigma}$. I will show in the forthcoming sections that, when dealing with noisy dynamic data, Calderón enrichment is not required if the scale $\sigma$ is chosen appropriately.

Each of the atoms in (5.2.1) are convoluted with both $\operatorname{sech}(x)$ and $1+$ $\tanh (x)$ to give a transformed dictionary $T_{\sigma}$ :

$$
T_{\sigma}=\left\{\operatorname{sech}\left(\frac{x-k \mu}{\sigma}\right) \star[\operatorname{sech}(x)+i(1+\tanh (x))], \quad k \in \mathbb{Z}\right\} .
$$

I shall choose atoms from $T_{\sigma}$ to fit the experimental values of the complex shear modulus. It is easily shown that the transformed dictionary $T_{\sigma}$ shares the same sampling rate $\mu$ as the dictionary $D_{\sigma}$. In particular,

$$
T_{\sigma}=\{\chi(x-k \mu), \quad k \in \mathbb{Z}\}
$$

where $\chi$ is a complex function whose real and imaginary parts are defined in (5.2.2). 


\subsubsection{Sparsity in redundant dictionaries}

Complex signals often include structures that are not well represented by a few vectors in any single basis. Bases such as Fourier series have a limited capacity of sparse expression. On the other hand, dictionaries with a large number of atoms offer a greater likelihood of representing a function to a given resolution in terms of a small number of elements.

In building an approximation to a given function, there are a selection of algorithms which can be used to select atoms from a dictionary. When the atoms chosen are linearly spaced then the approximation is said to be linear. On the other hand, if the spacing between atoms is irregular then the approximation is said to be non-linear.

In this chapter I consider $m$-sparse approximations for the continuous relaxation spectrum of the form

$$
\begin{aligned}
h(t) & =\sum_{k \in \Lambda_{m}} b_{k} \Gamma\left(\frac{t-x_{k}}{\sigma}\right), \quad\left\|\mathbf{b}_{0}\right\|=m, \\
\text { where } \Gamma(t) & =\operatorname{sech}(t) \\
\text { and } x_{k} & =x_{0}+k a .
\end{aligned}
$$

$h(t)$ is a linear combination of $m$ atoms chosen from $D_{\sigma} . x_{k}$ denotes the position of an atom; the integer $k$ labels the atom, and $k$ belongs to an index set $\Lambda_{m}$ with $m$ elements. For example, with $m=4$, one possible index set is $\Lambda_{m}=\{0,17,23,42\}$, which means that $h(t)$ would be constructed from four atoms centred at $x_{0}, x_{17}, x_{23}$ and $x_{42}$. I shall confine attention to a linear search algorithm, in which the spacing parameter $a$ in (5.2.6) is an integer multiple of the sampling rate $\mu$ of the dictionary. $x_{0}$ is the centre of the first selected basis function. I will give details of the search algorithm in Section 5.4. The approximation (5.2.4) has an associated parameter set $\left\{m, \sigma, a, x_{0}, b_{0}, \ldots, b_{m-1}\right\}$. This means that there are $m+4$ degrees of freedom in the approximation. 


\subsection{Regularization mechanisms.}

The key parameters in constructing an approximation for the continuous relaxation spectrum are $\sigma$ (which dictates the resolution of the primary approximation space $V_{0}$ ), $m$ (which is the number of basis functions selected from $V_{0}$ ) and $a$ (the spacing parameter of the representation).

The resolution, controlled by $\sigma$, is an extremely important factor in determining the sparsity of an approximation. If a low resolution approximation is sought after, which is associated with a large value of $\sigma$, and approximate $h$ from $V_{0}$ alone, then if $\sigma$ is too large it will be impossible to get a respectable fit to the data in terms of a positive relaxation spectrum. In particular, if the resolution is too low it is impossible to resolve peaks in the spectrum without losing positivity.

If a high resolution approximation is preferred, which is associated with small values of $\sigma$, and approximate $h$ from $V_{0}$ alone, then fewer basis elements can generally be used to obtain a very good fit to the data. However, decreasing $\sigma$ affects the smoothness of the spectrum and if $\sigma$ is too small, more peaks in the distribution of $h$ are obtained than can be physically justified. If $\sigma$ is too small, $h$ is represented by a sparse set of spikes, which is analogous to sparse spike deconvolution [53]. There is consequently a trade-off between smoothness of the spectrum and accuracy when fitting the data.

A good choice of $\sigma$ means that $h$ can be approximated from $V_{0}$ alone and Calderón enrichment is not required. I have found that when dealing with real data, if $\sigma$ is too large and Calderón enrichment is needed, this can give an excellent fit to the data, but can give rise to significant, and physically meaningless, negative lobes in the relaxation spectrum. I believe this occurs when the resolution of the spaces $W_{j}$ is too high for the level of noise in the data, and consequently I begin to fit the noise in the data. For the remainder of this thesis I will therefore aim to select an appropriate value of 
$\sigma$ for which Calderón enrichment is not necessary. From the Calderon-Mallat decomposition, it is evident that approximating $h$ from $V_{0}$ alone is equivalent to applying a low-pass filter to the true spectrum. This is analogous to other filtering methods, which were mentioned in the introduction to this chapter.

I have also found that increasing $m$ increases accuracy but reduces stability, and when $m$ gets too large I get noise amplification. Furthermore, I have discovered that $a$, the spacing parameter, can also act as a regularization mechanism. If $a$ is too small it can affect the numerical stability as you proceed along an algorithm, and if $a$ it is too large a respectable fit to the data cannot be achieved.

\subsection{Search Algorithm}

In general I assume that the data $G^{\prime}\left(\omega_{j}\right), G^{\prime \prime}\left(\omega_{j}\right)$ are measured at $N$ values of frequency $\omega_{j}, j=1, \ldots, N$. In this section I discuss a search algorithm which enables me to construct a least-squares fit to the data using atoms from the transformed dictionary $T_{\sigma}$. Since $T_{\sigma}$ is infinitely-dimensional I cannot search the whole of $T_{\sigma}$, and I restrict attention to a (large) $d$-dimensional sub-dictionary with $d \gg N \gg m$.

The $d$ atoms in the transformed dictionary $T_{\sigma}$ are evaluated at each of the $N$ dynamic data points to give a discrete representation of each atom in $\mathbb{R}^{N}$. This gives us a discrete sub-dictionary, which has the form of an $N \times d$ complex matrix $\chi$, i.e.

$$
\chi_{j k}=\chi\left(x_{j}-k \mu\right), \quad j=1, \ldots, N, k=L-d+1, \ldots, L,
$$

where $\chi$ was introduced in (5.2.3).

The search algorithm selects $m$ equally spaced columns of the matrix $\chi$ that give the best simultaneous least squares fit to the storage and loss 
moduli data. I do this by minimizing the weighted sum of squared residuals

$$
\sum_{j}\left[\frac{\Delta_{1}\left(x_{j}\right)}{G^{\prime}\left(\omega_{j}\right)}\right]^{2}+\sum_{j}\left[\frac{\Delta_{2}\left(x_{j}\right)}{G^{\prime \prime}\left(\omega_{j}\right)}\right]^{2},
$$

where

$$
\begin{aligned}
& \Delta_{1}\left(x_{j}\right)=G^{\prime}\left(\omega_{j}\right)-g_{1}\left(x_{j}\right), \\
& \Delta_{2}\left(x_{j}\right)=G^{\prime \prime}\left(\omega_{j}\right)-g_{2}\left(x_{j}\right),
\end{aligned}
$$

where $x_{j}=\ln \omega_{j}$ and

$$
\begin{aligned}
& g_{1}(x)=\sum_{k \in \Lambda_{m}} b_{k} \Gamma\left(\frac{x-x_{k}}{\sigma}\right) \star[1+\tanh (x)], \\
& g_{2}(x)=\sum_{k \in \Lambda_{m}} b_{k} \Gamma\left(\frac{x-x_{k}}{\sigma}\right) \star \operatorname{sech}(x) .
\end{aligned}
$$

I minimize the weighted sum of squared residuals for all possible choices within the dictionary for $m$ equally spaced atoms, and select the $m$ equally spaced atoms which give the lowest RMS error, with spacing $a$. This gives rise to an estimate for the continuous relaxation spectrum of the form

$$
\begin{aligned}
h(t) & =\sum_{k=0}^{m-1} b_{k} \Gamma\left(\frac{t-x_{k}}{\sigma}\right), \\
\text { where } \Gamma(t) & =\operatorname{sech}(t) \\
\text { and } x_{k} & =x_{0}+k a .
\end{aligned}
$$

For each value of $m$, the search algorithm calculates the least-squares fit to the data and subsequent RMS error for all possible values of $x_{0}$ and $a$ from the sub-dictionary. This parameter search is explained graphically in Fig. 5.1, where the vertical $z$-axis refers to the RMS error levels. The 
selected values of $x_{0}$ and $a$ are those that give the minimum RMS error for the search.

I compare the data fits and their associated relaxation spectra for different values of $m$ and $\sigma$. I select the smallest value of $m$ compatible with acceptable root mean square error (RMS) levels. For a chosen value of $m$ I will now compare results, for various values of $\sigma$, for a set of real data, to help establish methods for selecting the optimal value of $\sigma$.

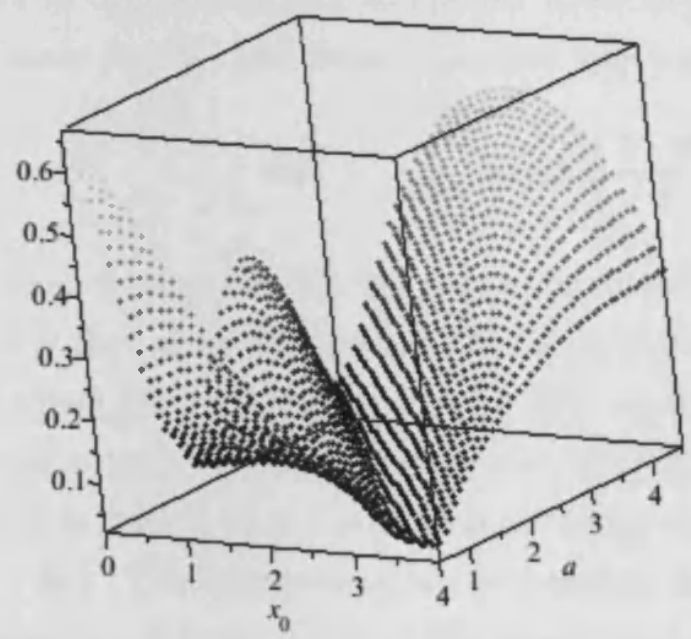

Figure 5.1: Parameter search. $z$-axis refers to the RMS errors. 


\subsection{PB1 Results}

In this section I use a set of data published by Honerkamp [43]. This data describes a polybutadiene polymer blend, which we called PB1. The values of $G^{\prime}$ and $G^{\prime \prime}$ are shown in Fig. 1.2 (p15); the frequency range is $2.493 \leq \omega \leq 1.114 \times 10^{3}$ and the corresponding range of $x=\ln \omega$ is $0.913 \leq x \leq 7.016$.

Honerkamp, using a discrete method based on Tikhonov regularization [43], concluded that this particular polymer blend has a bimodal relaxation spectrum. I shall now show that wavelet analysis confirms Honerkamp's conclusions that the relaxation spectrum is bimodal.

For this set of data I discovered, using my search algorithm, that the least value of $m$ compatible with acceptable RMS levels is $m=4$. This leads to an estimate for the continuous relaxation spectrum of the form

$$
h(t)=\sum_{k=0}^{3} b_{k} \operatorname{sech}\left(\frac{t-x_{k}}{\sigma}\right),
$$

where $x_{k}=x_{0}+k a$. For this $m$-sparse approximation, I found that the largest value of $\sigma$ that gives an acceptable fit to the storage and loss moduli data is $\sigma=\frac{1}{2}$ which gives an RMS error of $1.96 \%$, leading to a bimodal relaxation spectrum with a very small negative lobe (Fig. 5.2(a)). The relaxation spectrum is plotted on the same axis as the log-frequency axis, as a function of $t=-\ln \tau$. The spectrum shown is therefore the mirror image of the true spectrum as a function of $\ln \tau$. The spectrum in Fig. 5.2(a) has its larger peak centred at $\tau_{1}=1.4 \times 10^{-3}$ and the secondary peak at $\tau_{2}=1.6 \times 10^{-2}$. These results are not too dissimilar to those obtained by Honerkamp, whose has peaks centred at $\tau_{1}=8 \times 10^{-4}$ and $\tau_{2}=2 \times 10^{-2}$.

When I decrease the scaling parameter to $\sigma=\frac{1}{3}$ I start to get a trimodal spectrum (Fig. 5.2(b)), which is more prevalent when $\sigma=\frac{1}{4}$ (Fig. 5.2(c)). When $\sigma$ is small enough, e.g. $\sigma=\frac{1}{6}$ (Fig. 5.2(d)) I obtain an 4-mode 
distribution, giving an excellent fit to the data. Interestingly, the lowest RMS error is achieved when $\sigma=\frac{1}{6}$ (for $m=4$ ) and not when $\sigma=0$ (the discrete case (Fig. 5.2(e))), which shows that the best fit to the data is not achieved by a discrete relaxation spectrum. The parameter values of the $x_{0}$, $a$ and the coefficients $b_{k}$, are displayed in Table 5.1 for each value of $\sigma$.

All the spectra in Fig. 5.2 give estimates of the relaxation spectrum which result in acceptable fits to the data. From the point of view of fitting the data, there is very little to distinguish between them. Therefore, I must have an a priori selection criterion for choosing $\sigma$. The criterion I choose is minimum total curvature.

\begin{tabular}{|c|c|c|c|c|c|c|c|}
\hline$\sigma$ & $x_{0}$ & $a$ & $b_{0}$ & $b_{1}$ & $b_{2}$ & $b_{3}$ & RMS \\
\hline $1 / 2$ & 2.95 & 1.20 & -0.57 & 6.14 & -1.92 & 3.87 & 1.96 \\
\hline $1 / 3$ & 2.47 & 1.52 & -0.10 & 6.23 & 1.54 & 3.71 & 1.64 \\
\hline $1 / 4$ & 2.20 & 1.72 & -0.02 & 7.69 & 3.48 & 4.36 & 1.35 \\
\hline $1 / 6$ & 3.77 & 1.27 & 9.47 & 5.08 & 3.41 & 5.56 & 1.24 \\
\hline $1 / 7$ & 3.73 & 1.23 & 10.48 & 6.26 & 3.54 & 6.77 & 1.28 \\
\hline 0 & 3.61 & 1.16 & 3.96 & 3.34 & 1.12 & 3.34 & 1.42 \\
\hline
\end{tabular}

Table 5.1: Parameter values which minimise the RMS error for different values of $\sigma(m=4)$. The $b_{k}$ should be multiplied by $10^{5}$ for their true value.

\subsubsection{Minimum total curvature as a method for choos- ing $\sigma$.}

The relaxation spectrum $H(\tau)$ (or $h(t)$ ) is a density function describing the continuous distribution of relaxation times associated with a viscoelastic material. Density functions which occur frequently in nature are invariably smoath. Consequently, to choose a value of $\sigma$ for a fixed value of $m$, I select 




(a) $\sigma=\frac{1}{2}$, RMS error $=1.96 \%$.

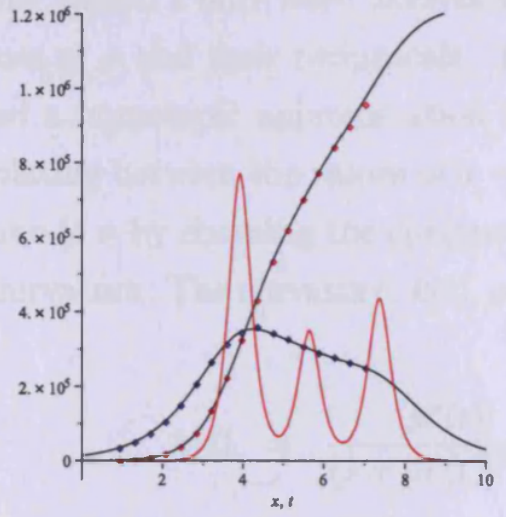

(c) $\sigma=\frac{1}{4}$, RMS error $=1.35 \%$.

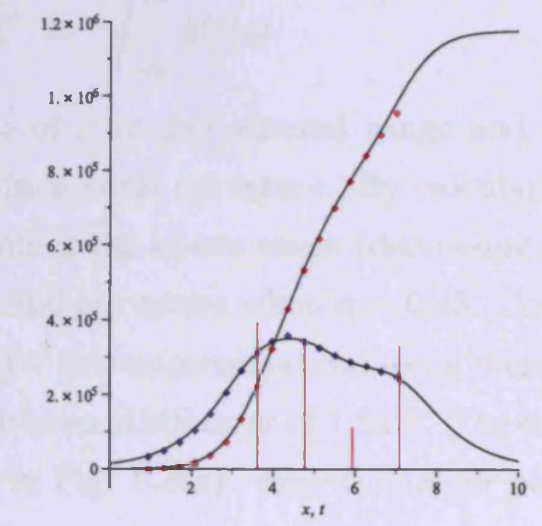

(e) $\sigma=0$, RMS error $=1.42 \%$.



(b) $\sigma=\frac{1}{3}$, RMS error $=1.64 \%$.

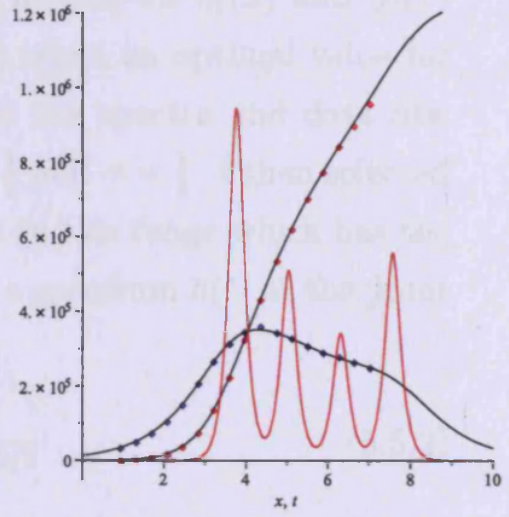

(d) $\sigma=\frac{1}{6}$, RMS error $=1.24 \%$.

Figure 5.2: Sparse approximation of PB1 using sech dictionary, with $m=4$. 
the smoothest spectrum that fits the data to an acceptable tolerance. This choice normally coincides with the smallest number of peaks that gives an acceptable fit to the data. As stated in the introductory section of this chapter, when dealing with dynamic data from an oscillatory shear experiment, levels of noise of around 1 or $2 \%$ are not unusual. I therefore perceive an acceptable fit to the data to have an RMS error less than $2 \%$. Consequently, I believe that this particular polymer is most likely to have a bimodal relaxation spectrum. Since $\sigma=\frac{1}{2}$ produces a bimodal spectrum with an unphysical negative lobe, and $\sigma=\frac{1}{3}$ produces a tri-modal spectrum, I infer from Table 5.1 that the optimal value of $\sigma$ is in the range $\frac{1}{3} \leq \sigma \leq \frac{1}{2}$.

So far in this thesis, I only have analytical models for $g_{1}(x)$ and $g_{2}(x)$ for integer values of $\sigma$ and their reciprocals. To select an optimal value for $\sigma$, I constructed a homotopic approximation for the spectra and data fits, linearly interpolating between the values of $\sigma=\frac{1}{2}$ and $\sigma=\frac{1}{3}$. I then selected the optimal value of $\sigma$ by choosing the spectrum in this range which has the smallest total curvature. The curvature, $k(t)$, of a spectrum $h(t)$ at the point $t$ is given by

$$
k(t)=\frac{\left|h^{\prime \prime}(t)\right|}{\left(1+\left[h^{\prime}(t)\right]^{2}\right)^{3 / 2}}
$$

and the total curvature of the spectrum, $T$, is given by

$$
T=\int_{-\infty}^{\infty} k(t) d t
$$

$T$ is calculated for each value of $\sigma$ in the selected range and I choose the value of $\sigma$ that gives the minimal total curvature. By calculating the total curvature, $T$, for each spectrum in the above range (decreasing $\sigma$ in steps of 0.01 from $\frac{1}{2}$ ), I get minimum total curvature when $\sigma=0.45$. Consequently, I believe that the optimal scale for this approximation, using merely four basis elements, is $\sigma=0.45$, which gives an RMS error of $1.84 \%$. The corresponding relaxation spectrum is shown in Fig. 5.3(a). Here the larger peak occurs at 
$\tau_{1}=1.2 \times 10^{-3}$ and the secondary peak at $\tau_{2}=1.7 \times 10^{-2}$, which are closer to the values obtained by Honerkamp, compared with the values for $\sigma=\frac{1}{2}$. This is illustrated in Fig. 5.3(b), where I have plotted Honerkamp's relaxation spectrum [43] against my normalized homotopic approximation, as an increasing function of $\ln \tau$. In order to compare my homotopic approximation with Honerkamp's spectrum, I have plotted $h(-t) / h_{0}$, where

$$
h_{0}=\int_{-\infty}^{\infty} h(t) d t \approx 1.19 \times 10^{6} .
$$

The vertical bars displayed in Honerkamp's spectrum denote error bars. The overall shape of the two spectra is similar, but an important advantage of my homotopic approximation over Honerkamp's spectrum is that it does not predict any negative lobes.
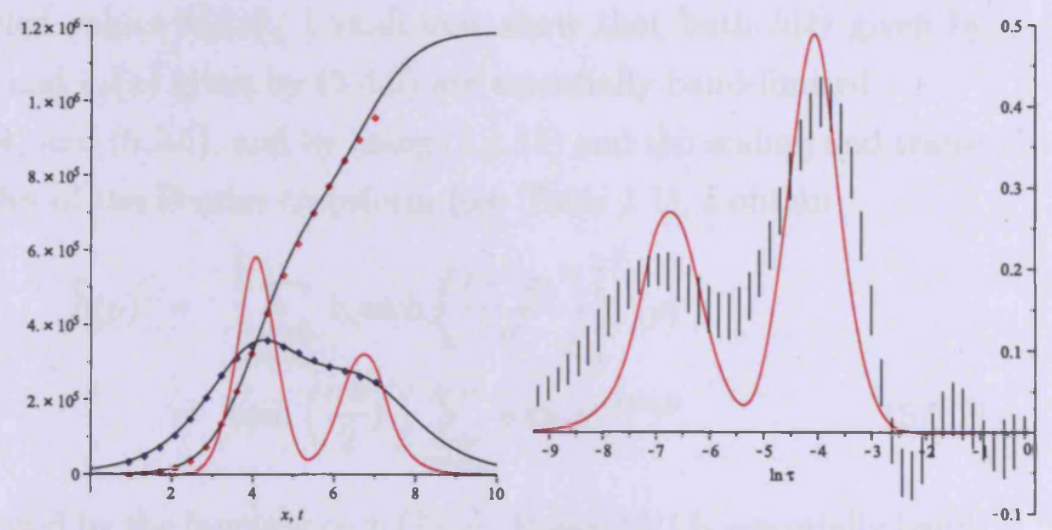

(a) Homotopic approximation. $\sigma=$ (b) Normalized homotopic approxima0.45 , RMS error $=1.84 \%$. tion against Honerkamp's spectrum.

Figure 5.3: Comparison of homotopic approximation and Honerkamp's spectrum. 


\subsubsection{Super-resolution}

The concept of super-resolution is another important factor in deciding upon an optimal value of $\sigma$.

Theorem 5.1: Shannon, Whittaker [53]. If the support of $\widehat{h}$ is included in $\left[-\frac{\pi}{s}, \frac{\pi}{s}\right]$, then

$$
h(t)=\sum_{n=-\infty}^{n=+\infty} h(n s) \Omega_{s}(t-n s)
$$

where

$$
\Omega_{s}(t)=\frac{\sin (\pi t / s)}{\pi t / s}
$$

$s$ is the sampling rate of the data and the range $\left[-\frac{\pi}{s}, \frac{\pi}{s}\right]$ is known as the Nyquist range of the data. This means that a band-limited function (one whose Fourier transform has finite support) can be recovered completely from its sampled values $h(n s)$. I shall now show that both $h(t)$ given by (5.2.4)-(5.2.6) and $g_{2}(x)$ given by (5.4.6) are essentially band-limited.

From (5.2.4) and (5.2.5), and by using (2.1.13) and the scaling and translation properties of the Fourier transform (see Table 1.1), I obtain

$$
\begin{aligned}
\widehat{h}(p) & =\left[\sum_{k \in \Lambda_{m}} b_{k} \operatorname{sech}\left(\frac{t-x_{k}}{\sigma}\right)\right]^{\wedge}(p) \\
& =\operatorname{sech}\left(\frac{\sigma \pi}{2} p\right) \sum_{k \in \Lambda_{m}} \sigma \pi b_{k} e^{-i \sigma x_{k} p},
\end{aligned}
$$

which is windowed by the function $\operatorname{sech}\left(\frac{\sigma \pi}{2} p\right)$. Hence $h(t)$ is essentially bandlimited. Furthermore, from (2.1.26), we have

$$
\widehat{h}(p)=\frac{1}{\pi} \widehat{g_{2}}(p) \cosh \left(\frac{\pi}{2} p\right) .
$$

Substituting the above equation into (5.5.6), I obtain

$$
\widehat{g_{2}}(p)=\operatorname{sech}\left(\frac{\pi}{2} p\right) \operatorname{sech}\left(\frac{\sigma \pi}{2} p\right) \sum_{k \in \Lambda_{m}} \sigma \pi^{2} b_{k} e^{-i \sigma x_{k} p}
$$


This shows that $\widehat{g_{2}}(p)$ is windowed by the function $\operatorname{sech}\left(\frac{\pi}{2} p\right) \operatorname{sech}\left(\frac{\sigma \pi}{2} p\right)$ and hence $g_{2}(x)$ is also essentially band-limited.

I can now address the question of resolution. If a function is band-limited or essentially band-limited, then its resolution is determined by its Nyquist bandwidth or its essential bandwidth. The larger the bandwidth (the broader the window) then the smaller the sampling interval in the Shannon-Whittaker theorem. High resolution therefore corresponds to a broad window, and low resolution to a narrow window. The window in (5.5.6) is broader than the window in (5.5.8), which means that the level of resolution obtained in approximating the spectrum is greater than the level of resolution obtained in approximating the loss modulus. Another way of stating this is to say that the resolving power of the dictionary $D_{\sigma}$ is greater than the resolving power of the dictionary $T_{\sigma}$. This is clearly evident from Figs. 5.2(a) and 5.3, where the two peaks are clearly resolved, whereas the two peak structure in $g_{2}(x)$ is hidden. It is interesting to observe that the functions $h$ and $g_{2}$ correspond to the same function resolved at different scales.

In the modern signal processing literature, e.g [53], the term superresolution seems to be widely used to describe the enhanced resolving power introduced in the previous paragraph. Formerly, this would have been described simply as resolution enhancement. In this thesis, I shall use the term super-resolution to describe spectral extrapolation beyond the Nyquist range of the data [1]. In particular, I shall say that super-resolution occurs when the Fourier transform of the recovered function $h(t)$ lies outside the Nyquist range of the data.

In this context, it will become clear that super-resolution can be controlled by the choice of the scaling parameter $\sigma$. Super-resolution occurs when not all of the area of the window function of $h$ is contained within the Nyquist range of the data. I can consider the amount of super-resolution to be the percentage of the area of the window function that lies outside this 
range. Then the amount of super-resolution for a particular value of $\sigma$ can be calculated as follows. Applying property (1.5.3) to equation (2.1.13), we see that

$$
\frac{\sigma}{2} \int_{-\infty}^{\infty} \operatorname{sech}\left(\frac{\sigma \pi}{2} p\right) d p=1
$$

The percentage of super-resolution, $S R$, for a particular value of $\sigma$ can then be expressed as

$$
S R=\left[1-\frac{\sigma}{2} \int_{-\frac{\pi}{3}}^{\frac{\pi}{3}} \operatorname{sech}\left(\frac{\sigma \pi}{2} p\right) d p\right] \times 100 \%,
$$

where $s$ is the sampling rate of the data.

It is clear from this definition that there will always be a certain amount of super-resolution. As the value of $\sigma$ decreases the basis functions for $h$ become narrower. As a consequence, its Fourier transform gets wider and the amount of super-resolution increases. This is displayed in Fig. 5.4, for a sampling rate $s=0.5$. When $\sigma=\frac{1}{2}$, over $99 \%$ of the area of the window function is confined to the Nyquist range. But, as $\sigma$ decreases, more and more of the window function lies outside the Nyquist range. When $\sigma=\frac{1}{8}$, less than $37 \%$ of the area of the window function is confined to the Nyquist range, and when $\sigma=\frac{1}{32}$, less than $10 \%$ of the area is confined to the range.

Since

$$
\lim _{\sigma \rightarrow 0} \frac{1}{\sigma} \operatorname{sech}\left(\frac{t}{\sigma}\right)=\delta(t)
$$

then in the limit as $\sigma \rightarrow 0$ the basis functions for $h$ become $\delta$-functions. Also, because

$$
\widehat{\delta(t)}(p)=1
$$

as $\sigma \rightarrow 0, \widehat{h}(p)$ has infinite support and is way beyond the limit of the sampling theorem. Consequently, here is another argument against using 


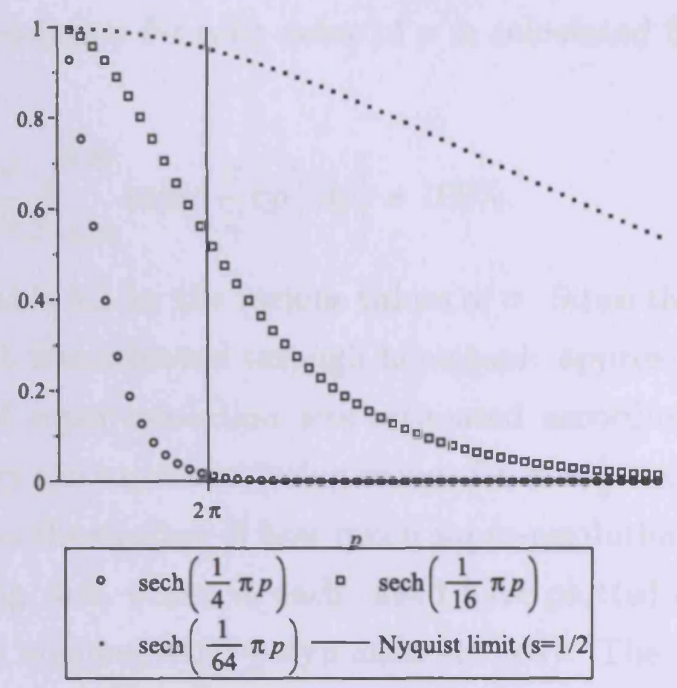

Figure 5.4: Window function for $\sigma=1 / 2, \sigma=1 / 8$ and $\sigma=1 / 32$ against Nyquist limit for $s=1 / 2$.

the $\delta$-functions as basis functions for $h$, since the value of $\sigma=0$ includes all frequencies and gives maximum (100\%) super-resolution.

Using the translation property of the Fourier transform (see Table 1.1), equation (5.5.6) can also be expressed as

$$
\widehat{h}(p)=\sigma \pi \operatorname{sech}\left(\frac{\sigma \pi}{2} p\right) \sum_{k \in \Lambda_{m}} b_{k}\left[\delta\left(t-\sigma x_{k}\right)\right]^{\wedge}(p) .
$$

Therefore $h(t)$ can be expressed in the form

$$
h(t)=\operatorname{sech}\left(\frac{t}{\sigma}\right) \star \sum_{k \in \Lambda_{m}} b_{k} \delta\left(t-\sigma x_{k}\right) .
$$

This shows that the approximation for $h(t)$ is just a set of $\delta$-functions windowed by a sech function.

The sampling rate of the PB1 data is approximately $s=0.38$ and hence the Nyquist limit of the data is $\pi / s \approx 8.24$. Consequently, using (5.5.10), 
the percentage of super-resolution for each value of $\sigma$ is calculated from the equation

$$
S R=\left[1-\frac{\sigma}{2} \int_{-8.24}^{8.24} \operatorname{sech}\left(\frac{\sigma}{2} \pi p\right) d p\right] \times 100 \% .
$$

The results are listed in Table 5.2 for the various values of $\sigma$. Since the recovered spectrum for $\sigma=0.45$ was achieved through homotopic approximation, the subsequent amount of super-resolution was estimated accordingly, linearly interpolating between the super-resolution values for $\sigma=\frac{1}{2}$ and $\sigma=\frac{1}{3}$. For different values of $\sigma$, an illustration of how much super-resolution that is taking place is given in Fig. 5.5, where in each case I have plotted $\widehat{h}(p)$ (in blue) and its unwindowed trigonometric polynomial (in red). The Nyquist range of the data is the interval between the two vertical bars. These graphs show that if $\sigma$ is too small then the effective bandwidth of the spectrum is wider than the Nyquist bandwidth of the data, resulting in significant super-resolution. When $\sigma=0$ there is no window function.

In general, when recovering the continuous relaxation spectrum from experimental data, I try to avoid super-resolution as much as possible. For the PB1 data, a value of $S R$ less than $1 \%$ would seem to be acceptable. By comparing Figs. 5.1 and 5.2 with Fig. 5.4 and Table 5.2, it can be seen that when a value of $\sigma$ leads to a value of $S R>1 \%$, there are more than two lobes in the relaxation spectrum. 


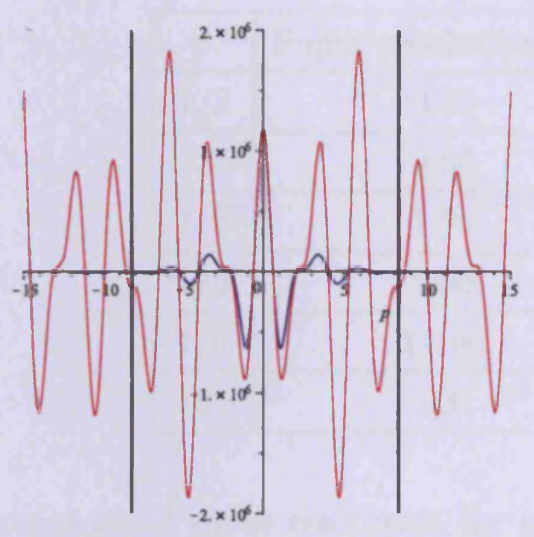

(a) $\sigma=\frac{1}{2}$.

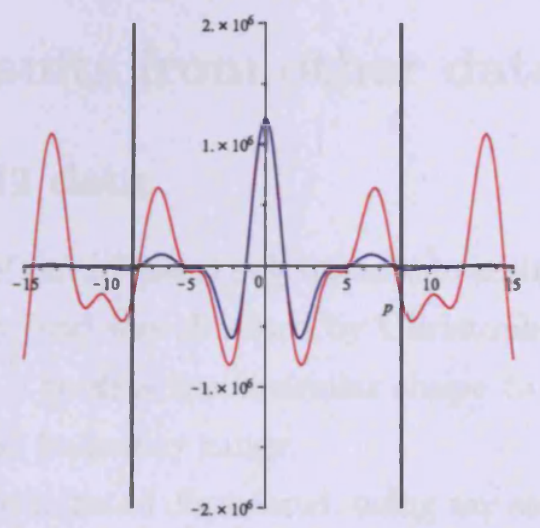

(c) $\sigma=\frac{1}{4}$.

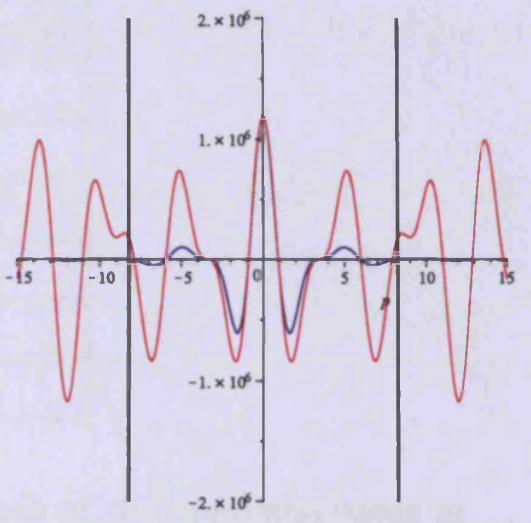

(b) $\sigma=\frac{1}{3}$.

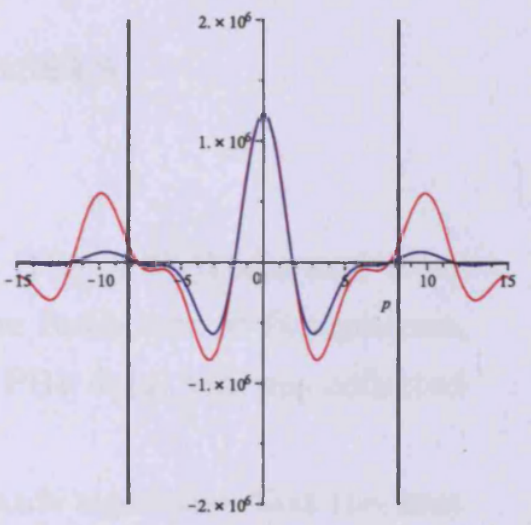

(d) $\sigma=\frac{1}{6}$.
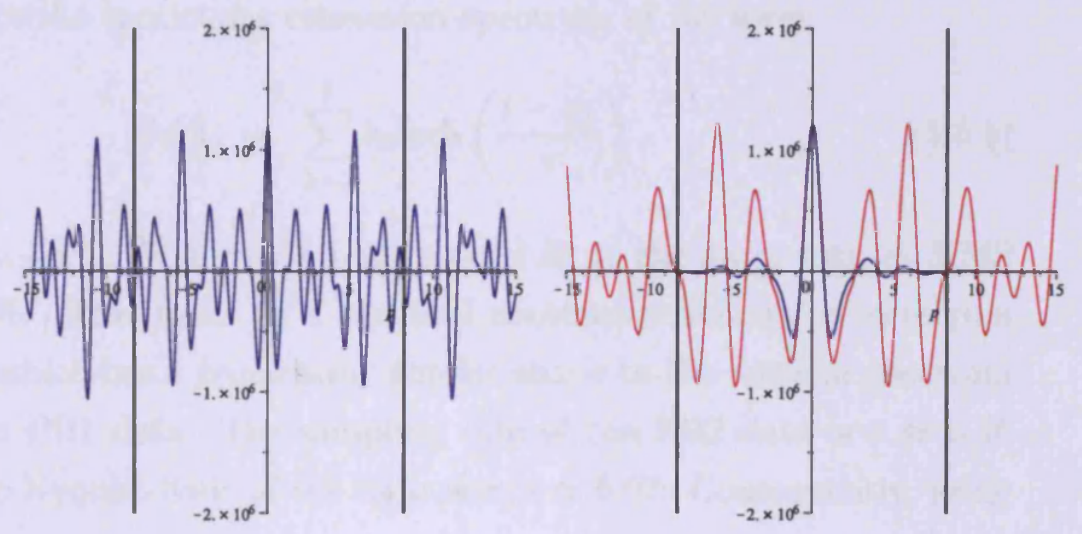

(e) $\sigma=0$.

(f) $\sigma=0.45$. Homotopic approximation.

Figure 5.5: Effect of windowing function. Trigonometric polynomial (red), $\widehat{h}(p)$ (blue) and the Nyquist bandwidth of the data (bold black). 


\begin{tabular}{|c|c|}
\hline$\sigma$ & Super-resolution (\%) \\
\hline $1 / 2$ & 0.20 \\
\hline 0.45 & 0.65 \\
\hline $1 / 3$ & 1.70 \\
\hline $1 / 4$ & 5.00 \\
\hline $1 / 6$ & 14.66 \\
\hline 0 & 100 \\
\hline
\end{tabular}

Table 5.2: Percentage of super-resolution for each of the approximations in Fig. 5.2.

\subsection{Results from other datasets}

\subsubsection{PB2 data}

This set of data from a polybutadiene melt (Fig. 5.6(a)) was sent to us by John Dealy, and was obtained by Christopher Robertson at Bridgestone, Akron, Ohio. The data has a similar shape to PB1 data, but was collected over a different frequency range.

For this set of data I discovered, using my search algorithm, that the least value of $m$ compatible with acceptable RMS levels is $m=5$. This leads to an estimate for the continuous relaxation spectrum of the form

$$
h(t)=\sum_{k=0}^{4} b_{k} \operatorname{sech}\left(\frac{t-x_{k}}{\sigma}\right),
$$

where $x_{k}=x_{0}+k a$. With $\sigma=\frac{1}{2}$ I get a good fit to the data, with an RMS error of $1.89 \%$. This leads to a bimodal continuous relaxation spectrum (Fig. 5.6(a)) which has a remarkably similar shape to the optimal spectrum obtained from PB1 data. The sampling rate of the PB2 data is $s \approx 0.46$ and hence the Nyquist limit of the data is $\pi / s \approx 6.82$. Consequently, using 
(5.5.10), the percentage of super-resolution for this optimal value of $\sigma$ is given by

$$
S R=\left[1-\frac{1}{4} \int_{-6.82}^{6.82} \operatorname{sech}\left(\frac{1}{4} \pi p\right) d p\right] \times 100 \% \approx 0.60 \%
$$

This means that over $99 \%$ of the area of $\widehat{h}(p)$ is contained within the Nyquist range of the data. This is also illustrated in Fig. 5.6(b).



(a) $\sigma=\frac{1}{2}$, RMS error $=1.89 \%$.

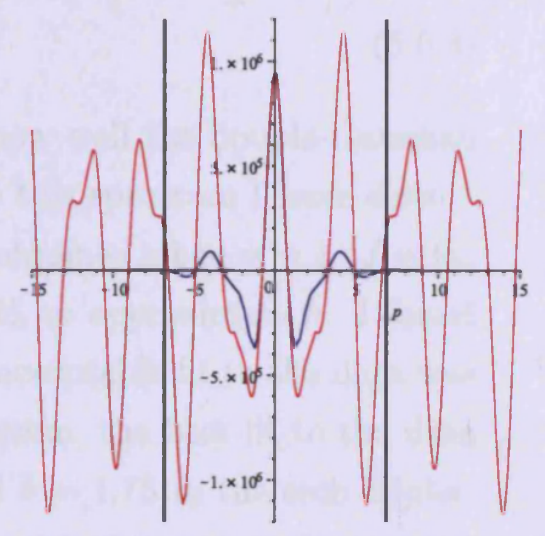

(b) Super-resolution.

Figure 5.6: Sparse approximation of PB2 relaxation spectrum with $m=5$ and $\sigma=\frac{1}{2}$ (a). Effect of windowing function (b); trigonometric polynomial (red), $\widehat{h}(p)$ (blue) and the Nyquist bandwidth of the data (bold black). 


\subsubsection{Honerkamp A}

Honerkamp A data is a set of synthetic data produced by Honerkamp [43]. $\mathrm{He}$ invented a bimodal spectrum (a double-Gaussian) and then obtained $G^{\prime}(\omega)$ and $G^{\prime \prime}(\omega)$ directly from it. A large amount of noise was added to the data (approximately $4 \%$ ). His spectrum is of the form

$$
h(t)=\frac{1}{2 \sqrt{2 \pi}}\left(\exp \left[-\frac{(t+\ln 20)^{2}}{2}\right]+\exp \left[-\frac{(t-\ln 5)^{2}}{2}\right]\right)
$$

I fitted this noisy data to my models to see how well the double-Gaussian spectrum could be recovered. To approximate this spectrum I have discovered that the most accurate reconstruction is obtained when $\sigma=1(J=0)$. For this value of $\sigma$, I require sech triplets in $V_{0}$ to approximate $h$. I found that the sparsest approximation that gave an acceptable fit to the data was achieved when $m=2$. Using our search algorithm, the best fit to the data was obtained with $a=4.64, x_{0}=-1.62$, and $b=1.75$ in the sech triplet. I obtained a fit to the data of $5.13 \%$, plotted with the recovered spectrum (blue) in Fig. 5.7(a). Although the above parameter values led to the best fit to the data, an improved reconstruction of the spectrum was discovered when using $a=4.57, x_{0}=-1.55$ and $b=1.95$ (see Fig. 5.7(c)). However, it was obtained through a poorer fit to the data, where the RMS error in the data was $5.58 \%$.

\section{Super-resolution and RTI wavelets}

When using $\sigma \geq 1$, we express the continuous relaxation spectrum as a sum of sech triplets, namely

$$
h(t)=\sum_{k \in \Lambda_{m}} d_{k}\left[\operatorname{sech}\left(\frac{t-x_{k}}{\sigma}\right)-\frac{1}{2} \operatorname{sech}\left(\frac{b}{\sigma}\right)\left\{\operatorname{sech}\left(\frac{t+b-x_{k}}{\sigma}\right)+\operatorname{sech}\left(\frac{t-b-x_{k}}{\sigma}\right)\right\}\right]
$$


Taking the Fourier transform of $h\left(\frac{b}{\sigma}\right)$ we obtain

$$
\widehat{h}(p)=\operatorname{sech}\left(\frac{\sigma \pi}{2} p\right)\left[1-\operatorname{sech}\left(\frac{b}{\sigma}\right) \cos (\sigma b p)\right] \sum_{k \in \Lambda_{m}} \sigma \pi b_{k} e^{-i \sigma x_{k} p}
$$

which is again a trigonometric polynomial, windowed by the function $\operatorname{sech}\left(\frac{\sigma \pi}{2} p\right)$.

The amount of super-resolution in both of these approximations is extremely small. The Fourier transforms of the approximations in Fig.'s 5.7(a) and 5.7(c) are plotted in Fig.'s 5.7(b) and 5.7(d), respectively. The sampling rate of the double Gaussian data is $s \approx 0.48$ and hence the Nyquist limit of the data is $\pi / s \approx 6.59$. The effect of the window function in both cases is substantial, as almost all of $\widehat{h}(p)$ is contained within the Nyquist range of the data. In Fig.'s 5.7(b) and 5.7(d), the trigonometric polynomials are plotted in red, $\widehat{h}(p)$ in blue and the Nyquist bandwidth of the data is plotted in bold black. Using (5.5.10), I can calculate the amount of super-resolution in each recovery. For both approximations, we have $S R \approx 0.0040 \%$, which is extremely small.

\subsubsection{Double Log-Normal Spectrum Approximation}

I now give another example of how well my models can fit synthetic data using our search algorithm. The double log-Normal distribution

$$
h(t)=\frac{1}{2 \sqrt{2 \pi}} e^{t}\left(\exp \left[-\frac{1}{8} t^{2}\right]+1000 \exp \left[-\frac{1}{8}(t+3 \ln 10)^{2}\right]\right)
$$

was used by Stadler \& Bailly [70]. To approximate this spectrum I have discovered that the most accurate reconstruction is obtained when $\sigma=\sigma_{0}=$ $2(J=0)$. Since $\sigma>1$, I require sech triplets in $V_{0}$ to approximate $h$. The data I was given did not contain noise, so I used an unweighted least squares 


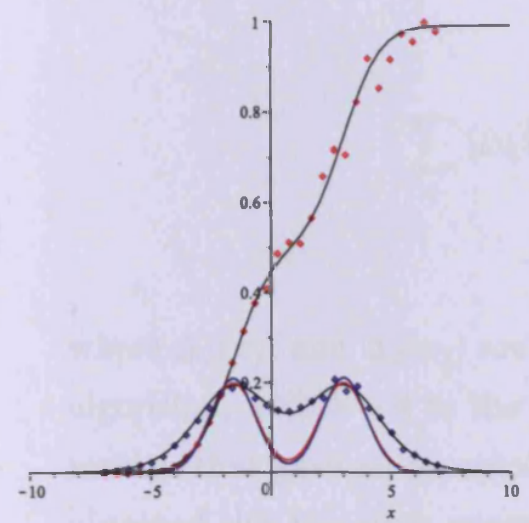

(a) Search algorithm results with minimum data RMS error.

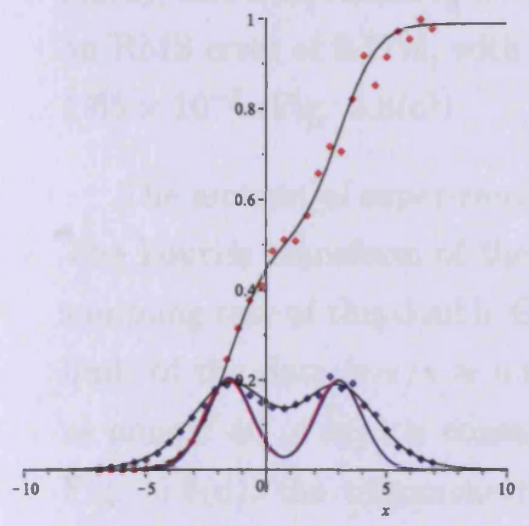

(c) Search algorithm results with best recovery.



(b) Effect of windowing function.

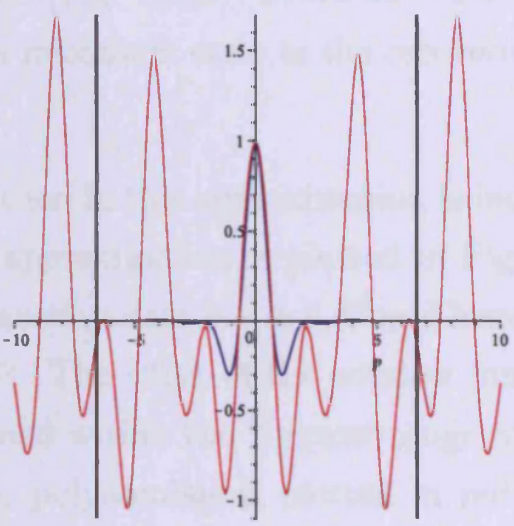

(d) Effect of windowing function.

Figure 5.7: Sparse approximation of Honerkamp A spectrum using noisy data with $m=2$ and $\sigma=1$. 
fit to the storage and loss data in the search algorithm, by mininmizing the unweighted sum of squared residuals

$$
\sum_{j}\left[\Delta_{1}\left(x_{j}\right)\right]^{2}+\sum_{j}\left[\Delta_{2}\left(x_{j}\right)\right]^{2}
$$

where $\Delta_{1}\left(x_{j}\right)$ and $\Delta_{2}\left(x_{j}\right)$ are defined in (5.4.3) and (5.4.4). Using my search algorithm, with $b=4$ in the sech triplet, I found that the sparsest approximation that gave an acceptable fit to the data was achieved with $m=2$. I obtained this two term approximation to the above spectrum with $a=6.91$ and $x_{0}=-4$. I obtained a fit to the data with an RMS error of $0.33 \%$ (Fig. 5.8(a)) and a recovered spectrum (Fig. 5.8(b)) with a fit to the Gaussian with an RMS error of $0.77 \%$, with a maximum error in the recovered spectrum of $1.65 \times 10^{-2}$ (Fig. 5.8(c)).

The amount of super-resolution in this approximation is incredibly small. The Fourier transform of the approximation is plotted in Fig. 5.8(d). The sampling rate of this double Gaussian data is $s \approx 0.47$ and hence the Nyquist limit of the data is $\pi / s \approx 6.63$. The effect of the window function is huge, as almost all of $\widehat{h}(p)$ is contained within the Nyquist range of the data. In Fig. 5.8(d), the trigonometric polynomials is plotted in red, $\widehat{h}(p)$ in blue and the Nyquist bandwidth of the data is plotted in bold black. Using (5.5.10), I can calculate the amount of super-resolution in the recovery as $S R \approx 1.16 \times 10^{-7} \%$.

Because there is no noise in the data, I could use Calderón enrichment to improve the accuracy of both the data fit and the spectrum recovery. However, since I can get such a good recovery of the double log-Normal spectrum using sech triplets alone, Calderón enrichment is not necessary. 

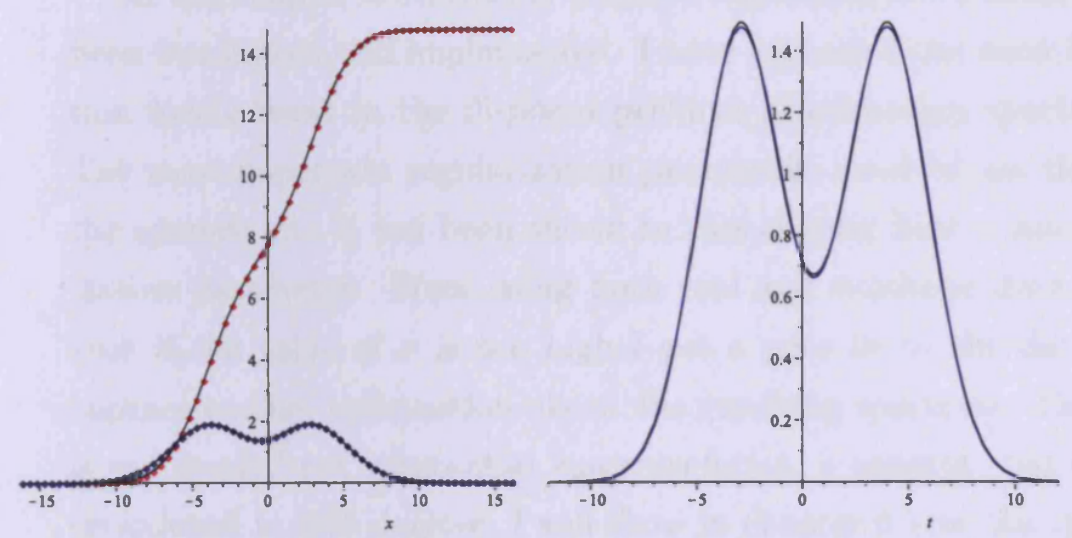

(a) $G^{\prime}(\omega)$ and $G^{\prime \prime}(\omega)$ data fit.

(b) Double Gaussian reconstruction.

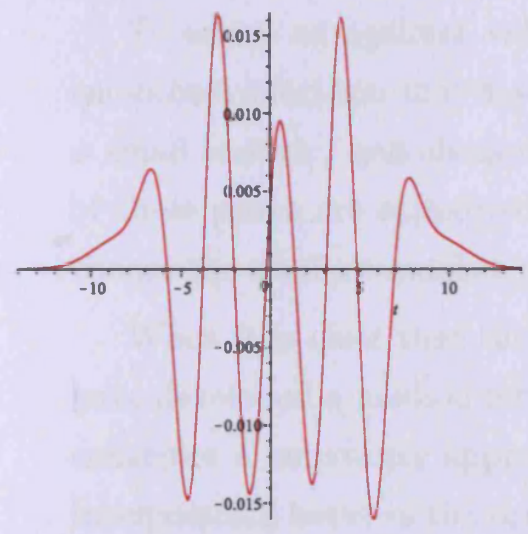

(c) Error in recovered spectrum.

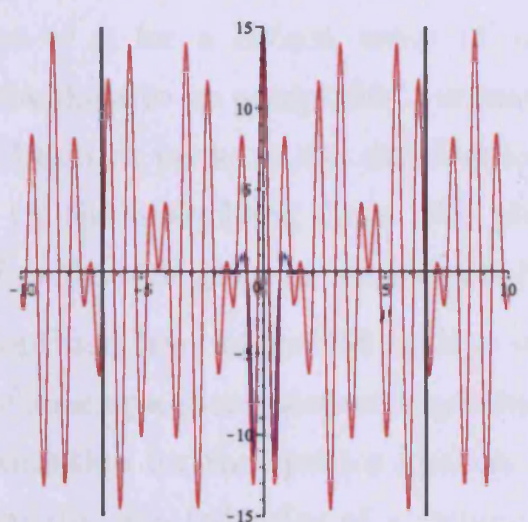

(d) Effect of window function.

Figure 5.8: Sparse approximation of double Gaussian spectrum using noisefree data, with $m=2$ and $\sigma=2$. 


\subsection{Conclusion}

In this chapter the concepts of sparse approximation and dictionaries have been introduced and implemented. I have explained the need for regularization mechanisms in the ill-posed problem of relaxation spectrum recovery. The most important regularization parameters involved are the scale $\sigma$ and the sparsity $m$. It has been shown in this chapter how $\sigma$ acts as a regularization parameter. From using both real and synthetic data, it is evident that if the value of $\sigma$ is too high I get a poor fit to the data and do not capture enough information about the resulting spectrum. Conversely, if $\sigma$ is too small I get substantial superresolution, a concept that has also been introduced in this chapter. I will show in chapter 6 how the sparsity $m$ and the spacing parameter $a$ act as regularization parameters.

To select an optimal value of $\sigma$ for a chosen value of $m$ I select the smoothest spectrum that fits the data to an acceptable tolerance. If I choose $\sigma$ small enough I can always obtain $m$ peaks in the distribution, but not all of these peaks are supported by the underlying data. My philosophy is to choose the smallest number of peaks that gives an acceptable fit to the data.

When it is clear that the optimal level of resolution is $\sigma \neq \frac{1}{n}(\sigma<1)$, I have developed a method for choosing a more accurate optimal value of $\sigma$. I construct a homotopic approximation for the spectra and data fits, linearly interpolating between the originally selected value of $\sigma$ (where the spectrum contains the smallest number of peaks that gives an acceptable fit to the data) and the nearest known smaller value of $\sigma$ (where there is at least one extra peak). I then select the optimal value of $\sigma$ by choosing the spectrum in this range which has the smallest total curvature.

I have introduced the concept of super-resolution, which is connected with part of the Fourier transform of the spectrum lying outside the Nyquist range of the data. When recovering the continuous relaxation spectrum 
from experimental data, I try to avoid super-resolution as much as possible. I believe that a value of super-resolution less than $1 \%$ is an acceptable value, since zero super-resolution is impossible when estimating the spectrum as a sum of hyperbolic wavelets.

I have given four sets of results for fitting data from real data, synthetic data with added noise, and synthetic noise-free data. I fitted the data using a basic search algorithm, which selects $m$ equally spaced basis functions to give the best simultaneous least squares fit to the storage and loss moduli data. In chapter 6 I will introduce other search and pursuit algorithms.

In this chapter we have focussed on a particular search algorithm. In Chapter 7, I will introduce other algorithms I have used in the recovery of the continuous relaxation spectrum. 
CHAPTER 5. 


\section{Chapter 6}

\section{Other Dictionaries}

So far in this thesis I have restricted attention to one dictionary - the sech-dictionary. In the current chapter I investigate other dictionaries in terms of whose atoms the relaxation spectrum can be expressed. There is a very important reason for considering more than one dictionary, namely, consistency of results. If similar estimates for the spectrum can be found by using more than one dictionary, then their consistency of representation enables greater confidence to be placed in the result.

I choose the sech-dictionary because, at scale $\sigma=1$, the Mallat term in the Calderón-Mallat decomposition is proportional to $G^{\prime \prime}(\omega)$. This enables me to present the Calderón term as a correction term for the FK approximation for the spectrum. A natural extension of the sech-dictionary is the $\operatorname{sech}^{n}$-dictionary ( $n$-th power of sech). These dictionaries enable me to find correction terms for higher order differentiation formulae (due to Schwarzl and Staverman [67], and Tschoegl [74]). The $\operatorname{sech}^{n}$-dictionaries are derived in Section 6.2.

In this chapter I will focus on constructing wavelet dictionaries based on the Mallat part of the Calderón-Mallat decomposition. This means that the continuous relaxation spectrum is represented by father wavelets alone (as 
was shown in Chapter 5).

In Section 6.1 I give an example of a sub-hyperbolic dictionary, namely the $x$ cosech dictionary. The factor $x$ means that the atoms have a slightly slower rate of decay than exponential - hence sub-hyperbolic. In Section 6.3 I give examples of super-hyperbolic dictionaries with atoms of the form $x^{-1} \tanh (x) \operatorname{sech}^{2 n-1}(x)$.

\subsection{The $x$ cosech dictionary}

In Chapter 3 I introduced the scaling function $\phi^{\star}(x)=\operatorname{sech}(x)$, which was then used in the recovery of the continuous relaxation spectrum. If

$$
\phi(x)=\operatorname{sech}(x)
$$

then from Table 4.2, I obtain

$$
\begin{aligned}
\phi^{\star}(x) & =\operatorname{sech}(x) \star \operatorname{sech}(x) \\
& =2 x \operatorname{cosech}(x) .
\end{aligned}
$$

$\operatorname{sech}(x)$ and its autoconvolution $2 x \operatorname{cosech}(x)$ are plotted together in Fig. 6.1, where both functions have been normalized to have unit area. The autoconvolution of $\operatorname{sech}(x)$ is a broader function than $\operatorname{sech}(x)$. The factor $x$ means that the scaling function $2 x \operatorname{cosech}(x)$ has a slightly slower rate of decay than $\operatorname{sech}(x)$, which has exponential decay. Hence I call $x \operatorname{cosech}(x)$ a sub-hyperbolic father wavelet. Using the convolution property of Fourier transforms from Table 1.1, the Fourier transform of $\phi^{\star}(x)$ is given by

$$
\widehat{\phi^{\star}}(p)=\pi^{2} \operatorname{sech}^{2}\left(\frac{\pi}{2} p\right) \text {. }
$$

The cross-paired mother wavelet for $\phi^{\star}(x)$ is given by

$$
\begin{aligned}
\psi^{\star}(x) & =\frac{d}{d x}\left[x \phi^{\star}(x)\right] \\
& =2 x \operatorname{cosech}(x)[2-x \operatorname{coth}(x)]
\end{aligned}
$$




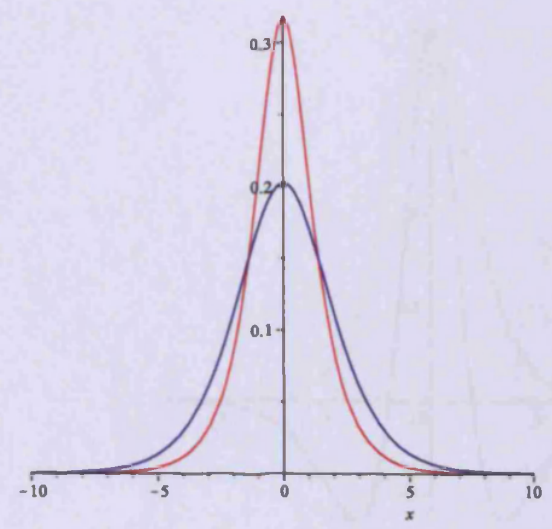

Figure 6.1: $x \operatorname{cosech}(x)$ (blue) against $\operatorname{sech}(x)$ (red), where both functions have been normalized to have unit area.

These cross-paired wavelets are plotted below in Fig. 6.2. Using the theory behind Section 3.5, it is not possible to give a closed form for the conventional mother wavelet pairing, $B(x)$, for $\phi^{\star}(x)=2 x \operatorname{cosech}(x)$. However, in a similar fashion to Section 3.5, I can compare the two mother wavelets in Fourier space. Using Table 1.1, we derive

$$
\begin{aligned}
\widehat{\psi^{\star}}(p) & =\left[\frac{d}{d x}(x[\operatorname{sech}(x) \star \operatorname{sech}(x)])\right]^{\wedge}(p) \\
& =-p \frac{d}{d p}[\widehat{\operatorname{sech}}(p)]^{2} \\
& =-\pi^{2} p \frac{d}{d p} \operatorname{sech}^{2}\left(\frac{\pi}{2} p\right) \\
& =\pi^{3} p \operatorname{sech}^{2}\left(\frac{\pi}{2} p\right) \tanh \left(\frac{\pi}{2} p\right)
\end{aligned}
$$




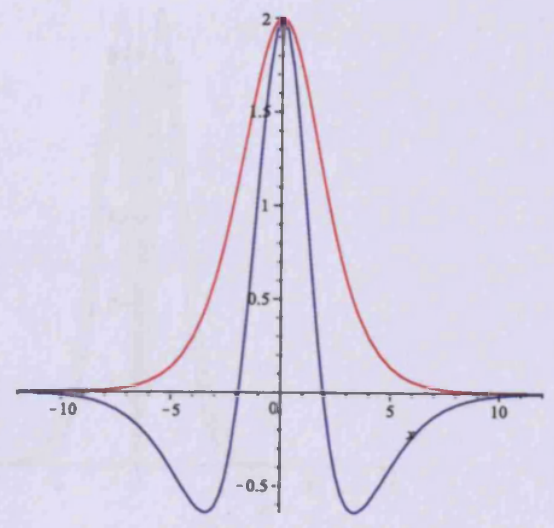

Figure 6.2: $\phi^{\star}(x)$ (red) against $\psi^{\star}(x)$ (blue).

Furthermore

$$
\begin{aligned}
\widehat{B}^{2}(p) & =\left[\frac{d}{d x}(x[\operatorname{sech}(x) \star \operatorname{sech}(x) \star \operatorname{sech}(x) \star \operatorname{sech}(x)])\right]^{\wedge}(p) \\
& =-p \frac{d}{d p}[\widehat{\operatorname{sech}}(p)]^{4} \\
& =-\pi^{4} p \frac{d}{d p} \operatorname{sech}^{4}\left(\frac{\pi}{2} p\right) \\
& =2 \pi^{5} p \operatorname{sech}^{4}\left(\frac{\pi}{2} p\right) \tanh \left(\frac{\pi}{2} p\right) \\
\therefore \widehat{B}(p) & =\sqrt{2} \pi^{\frac{5}{2}} \operatorname{sech}^{2}\left(\frac{\pi}{2} p\right) \sqrt{p \tanh \left(\frac{\pi}{2} p\right)}
\end{aligned}
$$

These two functions derived in (6.1.5) and (6.1.6) are plotted below in Fig. 6.3 , to compare the two mother wavelets in the Fourier domain.

I will now show that the cross-paired wavelets $\left(\psi^{\star}, \phi^{\star}\right)$ defined in this chapter satisfy the respective constraints of wavelet frames and Riesz bases. I will compare results with those obtained from the cross-paired wavelets introduced in Chapter 3. 




Figure 6.3: $\widehat{B}(p)$ (blue) against $\widehat{\psi^{\star}}(p)$ (red).

\subsubsection{Wavelet frames}

Definitions. Let $\Psi^{\star}$ denote the set $\left\{\psi_{j, k}^{\star}\right\}_{(j, k) \in \mathbb{Z}^{2}}$, where

$$
\psi^{\star}(x)=2 x \operatorname{cosech}(x)[2-x \operatorname{coth}(x)]
$$

Let $\mathbf{B}^{\star}$ denote the set $\left\{B_{j, k}\right\}_{(j, k) \in \mathbb{Z}^{2}}$, where

$$
B(x) \star B(x)=\frac{d}{d x}(x[\operatorname{sech}(x) \star \operatorname{sech}(x) \star \operatorname{sech}(x) \star \operatorname{sech}(x)]) .
$$

Let the sets $\Psi, \mathbf{B}$ and $\mathbf{M}$ be defined as they were in Section 3.6.

Theorem 6.1. The sets $\Psi^{\star}$ and $\mathbf{B}^{\star}$ each define frames of $L^{2}(\mathbb{R})$ for a sampling rate $a \leq 2$.

Theorem 6.2. With a sampling rate $a \leq \frac{1}{2}$, frame $\mathbf{B}$ is tighter than frame $\boldsymbol{\Psi}$, which is tighter than frame $\mathbf{B}^{\star}$, which is tighter than frame $\boldsymbol{\Psi}^{\star}$, which is tighter than frame $\mathbf{M}$. 
Theorem 6.3. With a sampling rate $\frac{3}{2} \leq a \leq 2$, frame $\mathbf{B}^{\star}$ is tighter than frame $\boldsymbol{\Psi}^{\star}$, which is tighter than frame $\mathbf{B}$, which is tighter than frame $\mathbf{M}$, which is tighter than frame $\boldsymbol{\Psi}$.

Remark: Theorem 6.1 is also true for certain sample rates $a>2$. In particular, there exist threshold values of $a$, namely $a_{\psi^{\star}}>2$ and $a_{B^{\star}}>2$, for which

(i) $\Psi^{\star}$ is a frame for all $0<a<a_{\psi^{\star}}$;

(ii) $\mathbf{B}^{\star}$ is a frame for all $0<a<a_{B^{\star}}$.

I have used Daubechies' necessary and sufficient conditions (equations (3.6.4)-(3.6.7)) to calculate the frame bounds for $\boldsymbol{\Psi}^{\star}$ and $\mathbf{B}^{\star}$, for certain values of the sampling rates, and with normalizations $\left\|\psi^{\star}\right\|_{2}=\|B\|_{2}=1$. These are shown in Tables 6.1 and 6.2. Theorems 6.1, 6.2 and 6.3 are now easily established by viewing the frame bounds shown in these tables, and from the frame bounds shown in Tables 3.1-3.3.

\begin{tabular}{|c|c|c|c|}
\hline sampling rate $(a)$ & $A_{0}$ & $B_{0}$ & $B_{0} / A_{0}$ \\
\hline 2 & 6.915 & 7.250 & 1.048 \\
\hline $3 / 2$ & 9.369 & 9.517 & 1.016 \\
\hline 1 & 14.067 & 14.261 & 1.014 \\
\hline $1 / 2$ & 28.135 & 28.521 & 1.014 \\
\hline $1 / 4$ & 56.270 & 57.043 & 1.014 \\
\hline
\end{tabular}

Table 6.1: Estimated frame bounds for the hyperbolic wavelet $\psi_{j, k}^{\star}(x)$, defined in (6.1.7). 


\begin{tabular}{|c|c|c|c|}
\hline sampling rate $(a)$ & $A_{0}$ & $B_{0}$ & $B_{0} / A_{0}$ \\
\hline 2 & 10.597 & 10.762 & 1.016 \\
\hline $3 / 2$ & 14.213 & 14.265 & 1.004 \\
\hline 1 & 21.326 & 21.390 & 1.003 \\
\hline $1 / 2$ & 42.653 & 42.780 & 1.003 \\
\hline $1 / 4$ & 85.305 & 85.561 & 1.003 \\
\hline
\end{tabular}

Table 6.2: Estimated frame bounds for the hyperbolic wavelet $B_{j, k}(x)$, defined in (6.1.8).

\subsubsection{Riesz basis}

The set $\left\{\phi_{J, k}^{\star}\right\}_{k \in \mathbf{Z}}$, where

$$
\phi^{\star}(x)=2 x \operatorname{cosech}(x),
$$

is a Riesz basis of the space $V_{J} \subset L^{2}(\mathbb{R})$. In particular, the set $\left\{\phi_{0, k}^{\star}\right\}_{k \in \mathbf{Z}}$ is a Riesz basis of the space $V_{0}$ it generates, since

$$
\forall \omega \in[-\pi, \pi], \quad 8.56 \times 10^{-8} \leq \sum_{k=-\infty}^{\infty}\left|\widehat{\phi^{\star}}(\omega-2 k \pi)\right|^{2} \leq \beta,
$$

where $\beta=1$ to 15 decimal places. The above basis has been normalised in the sense that $\left\|\phi_{0, k}^{\star}\right\|=1$. When compared to the Riesz bounds for the sech basis in Section 3.6, it is clear that in the above case the lower bound is much closer to zero and the upper bound is much closer to 1 . This is because the autoconvolution of $\operatorname{sech}(x)$ is wider than sech itself and, consequently, the Fourier transform of the autoconvolution is narrower than the Fourier transform of sech itself. Therefore the wider the basis function $\phi^{\star}(x)$ the smaller the essential support of the Fourier transform and the closer the lower and upper Riesz bounds get to 0 and 1, respectively. To emphasize this point further, if I choose an even wider basis function, e.g.

$$
\phi^{\star}(x)=\operatorname{sech}(x) \star \operatorname{sech}(x) \star \operatorname{sech}(x),
$$


then the set $\left\{\phi_{0, k}^{\star}\right\}_{k \in \mathbf{Z}}$ is a Riesz basis of the space $V_{0}$, the subsequent lower Riesz bound of $1.77 \times 10^{-11}$ is even closer to zero and the upper Riesz bound is equal to 1 to a staggering 23 decimal places. For consistency, in all of the calculations of the Riesz bounds I have kept to 40-digit arithmetic on Maple.

\subsubsection{Two-scale relations}

The cross-paired wavelets $\left\{\psi^{\star}, \phi^{\star}\right\}$, defined by (6.1.4) and (6.1.2) do not satisfy the two-scale relationships (3.7.5) and (3.7.6). Nevertheless, the crosspaired wavelets $\left\{\psi^{\star}, \phi^{\star}\right\}$ do satisfy equations (3.7.5) and (3.7.6) in an approximate sense. In particular, if the coefficients $\left\{a_{k}, b_{k}\right\}$ are chosen as the Fourier coefficients given in (3.7.11) and (3.7.12) then, although the residuals in the two-scale relations will not tend to zero as $k \rightarrow \infty$, they will be extremely small. Using (3.7.20) and (6.1.3), it can be shown that

$$
\begin{aligned}
\|\epsilon\|_{2}^{2} & =\frac{\pi^{3}}{2} \sum_{n=-\infty}^{n=\infty} \int_{-2 \pi}^{2 \pi}\left[\operatorname{sech}^{2}\left(\frac{\pi}{2}[p+4 \pi n]\right)-\operatorname{sech}^{2}\left(\frac{\pi}{4}[p+4 \pi n]\right) \frac{\operatorname{sech}^{2}\left(\frac{\pi}{2} p\right)}{\operatorname{sech}^{2}\left(\frac{\pi}{4} p\right)}\right]^{2} d p \\
& <2 \times 10^{-7}
\end{aligned}
$$

Similarly, using equation (3.7.23) and (6.1.5), I obtain

$$
\begin{aligned}
\|\rho\|_{2}^{2}= & \frac{\pi^{5}}{2} \sum_{n=-\infty}^{n=\infty} \int_{-2 \pi}^{2 \pi}\left\{(p+4 \pi n) \operatorname{sech}^{2}\left(\frac{\pi}{2}[p+4 \pi n]\right) \tanh \left(\frac{\pi}{2}[p+4 \pi n]\right)\right. \\
& \left.-\operatorname{sech}^{2}\left(\frac{\pi}{4}[p+4 \pi n]\right) \frac{p \operatorname{sech}^{2}\left(\frac{\pi}{2} p\right) \tanh \left(\frac{\pi}{2} p\right)}{\operatorname{sech}^{2}\left(\frac{\pi}{4} p\right)}\right\}^{2} d p \\
< & 2 \times 10^{-6} .
\end{aligned}
$$

This shows that the new cross-pairing of wavelets, derived in this chapter, give a smaller error in the two-scale relations than the original cross-pairing of wavelets derived in Chapter 3. Consequently, this new wavelet pairing can also be used to construct an essential multiresolution analysis. 


\subsubsection{Real-time integrability}

For the father wavelet $\phi^{\star}(t)=t \operatorname{cosech}\left(\frac{t}{\sigma}\right)$ to be RTI, I require $\sigma$ such that

$$
\int_{-\infty}^{\infty} t \operatorname{cosech}\left(\frac{t}{\sigma}\right) e^{-t} d t<\infty
$$

Manipulating the integrand of (6.1.13), I obtain

$$
t \operatorname{cosech}\left(\frac{t}{\sigma}\right) e^{-t}=2 t \exp \left[t\left(\frac{1}{\sigma}-1\right)\right]\left(\exp \left[\frac{2 t}{\sigma}\right]-1\right)^{-1} .
$$

To avoid the integrand becoming infinitely large as $t \rightarrow-\infty$, I require $0<$ $\sigma<1$. Thus, $\phi^{\star}(t)$ is RTI when $0<\sigma<1$. Similarly for the mother wavelet $\psi^{\star}(t)=t \operatorname{cosech}\left(\frac{t}{\sigma}\right)\left[2-\frac{t}{\sigma} \operatorname{coth}\left(\frac{t}{\sigma}\right)\right]$ to be RTI, I require $\sigma$ such that

$$
\int_{-\infty}^{\infty} t \operatorname{cosech}\left(\frac{t}{\sigma}\right)\left[2-\frac{t}{\sigma} \operatorname{coth}\left(\frac{t}{\sigma}\right)\right] e^{-t} d t<\infty
$$

Again this constraint is fulfilled when $0<\sigma<1$. Consequently, the basis elements in (6.1.16) are only RTI when $0<\sigma<1$. Since $t \operatorname{cosech}\left(\frac{t}{\sigma}\right)$ is a much wider function than $\operatorname{sech}\left(\frac{t}{\sigma}\right)$ for the same value of $\sigma$, I have not required a value of $\sigma>\frac{1}{2}$ in the new basis functions for the data I have utilized. I have therefore had no reason to investigate whether there is a subspace of the Riesz basis $V_{0}$ which is RTI for $\sigma>1$.

Using the definition of $\phi_{j, k}^{\star}$ in (3.8.2), I keep $V_{0}$ as our primary subspace, by varying $\sigma_{0}$ accordingly. I have now satisfied the necessary constraints to enable me to obtain a representation for $h(t)$ which resembles a discrete form of the Calderón-Mallat decomposition

$$
h(t) \approx \sum_{k} c_{k} \phi_{0, k}^{\star}(t)+\sum_{\substack{j \\ j>0}} \sum_{k} d_{j, k} \psi_{j, k}^{\star}(t), \quad j, k \in \mathbf{Z}, \sigma_{0} \in \mathbf{R} .
$$




\subsubsection{Transformed dictionaries}

In a similar fashion to Chapter 4 , I form models for $g_{1}(x)$ and $g_{2}(x)$, in order to fit the storage and loss moduli data. The above estimates for $h$ are convoluted with both $\operatorname{sech}(x)$ and $1+\tanh (x)$ to form atoms of a transformed dictionary $\chi$.

\section{Scaling function convolutions for $G^{\prime}$}

To develop the part of the model for $g_{1}(x)$ emanating from the scaling function part of $h$, I require the following convolutions for different values of $\sigma:$

$$
\begin{aligned}
& 2 x \operatorname{cosech}\left(\frac{x}{\sigma}\right) \star[1+\tanh (x)] \\
= & \operatorname{sech}\left(\frac{x}{\sigma}\right) \star \operatorname{sech}\left(\frac{x}{\sigma}\right) \star[1+\tanh (x)] \\
= & \left(\left[\operatorname{sech}\left(\frac{x}{\sigma}\right)\right]^{\wedge}\right)^{2}(0)+\operatorname{sech}\left(\frac{x}{\sigma}\right) \star \operatorname{sech}\left(\frac{x}{\sigma}\right) \star \tanh (x) \\
= & (\sigma \pi)^{2}+\operatorname{sech}\left(\frac{x}{\sigma}\right) \star \operatorname{sech}\left(\frac{x}{\sigma}\right) \star \tanh (x) .
\end{aligned}
$$

The convolution

$$
\operatorname{sech}\left(\frac{x}{\sigma}\right) \star \operatorname{sech}\left(\frac{x}{\sigma}\right) \star \tanh (x)
$$

can be obtained from the convolution theorem for Fourier transforms using the distribution theory developed in section 2.1. As a consequence of (2.1.22), I can write

$$
[\tanh (x)]^{\wedge}(p)=-i \pi \operatorname{cosech}\left(\frac{\pi}{2} p\right)
$$

and

$$
\begin{aligned}
{\left[\operatorname{sech}\left(\frac{x}{\sigma}\right) \star \operatorname{sech}\left(\frac{x}{\sigma}\right) \star \tanh (x)\right]^{\wedge}(p) } & =\left(\left[\operatorname{sech}\left(\frac{x}{\sigma}\right)\right]^{\wedge}\right)^{2}(p)[\tanh (x)]^{\wedge}(p) \\
& =-i \sigma^{2} \pi^{3} \operatorname{sech}^{2}\left(\frac{\sigma \pi}{2} p\right) \operatorname{cosech}\left(\frac{\pi}{2} p\right)
\end{aligned}
$$


By taking the inverse Fourier transform I obtain

$\operatorname{sech}\left(\frac{x}{\sigma}\right) \star \operatorname{sech}\left(\frac{x}{\sigma}\right) \star \tanh (x)=-\frac{(\sigma \pi)^{2}}{2} i \int_{-\infty}^{\infty} \frac{e^{i p x}}{\cosh ^{2}\left(\frac{\sigma \pi}{2} p\right) \sinh \left(\frac{\pi}{2} p\right)} d p$

I shall now calculate this integral for different values of $\sigma$. Let

$$
f(z)=\frac{e^{i x z}}{\cosh ^{2}\left(\frac{\sigma \pi}{2} z\right) \sinh \left(\frac{\pi}{2} z\right)}
$$

Using (4.2.9) and (4.2.10), it can be seen that $f(z)$ has simple poles at $z=$ $2 m i, \quad m \in \mathbb{N}$, and double poles at $z=\frac{2 m-1}{\sigma} i, \quad m \in \mathbb{N}^{+}$

Case (i): $\quad \sigma=\frac{1}{n}, \quad n=2 m, \quad m \in \mathbb{N}^{+}$

I use the indented contour displayed in Fig 4.1. $f(z)$ has $n$ simple poles at $z=2 m i\left(m \in \mathbb{N} \backslash\left\{\frac{n}{2}\right\}, m \leq n\right)$, and one pole of order 3 at $z=n i$. Let $f(z)=\frac{g(z)}{h(z)}$, where

$$
\begin{aligned}
& g(z)=\frac{e^{i x z}}{\cosh ^{2}\left(\frac{\pi}{2 n} z\right)} \\
& h(z)=\sinh \left(\frac{\pi}{2} z\right)
\end{aligned}
$$

Using (4.2.13), I find that the residue at the simple poles of $f(z)$ is

$$
\operatorname{Res}\{f(z) ; 2 m i\}=\frac{2}{\pi}(-1)^{m} \frac{e^{-2 m x}}{\cos ^{2}\left(\frac{m}{n} \pi\right)}
$$

Since there is a triple pole at $z=n i$, I consider a Laurent expansion of $f(z)$ 
at $z=n i$. Using equations (4.2.14)-(4.2.16), this gives

$$
\begin{aligned}
& \frac{8 n^{2}}{\pi^{3}}(-1)^{\frac{n}{2}+1} \frac{e^{-n x}\left[1+i x(z-n i)-\frac{1}{2} x^{2}(z-n i)^{2}+\ldots\right]}{(z-n i)^{3}\left[1+\frac{\pi^{2}}{24}(z-n i)^{2}+\ldots\right]\left[1+\frac{1}{3}\left(\frac{\pi}{2 n}\right)^{2}(z-n i)^{2}+\ldots\right]} \\
= & \frac{8 n^{2}}{\pi^{3}}(-1)^{\frac{n}{2}+1} \frac{e^{-n x}\left[1+i x(z-n i)-\frac{1}{2} x^{2}(z-n i)^{2}+\ldots\right]}{(z-n i)^{3}\left[1+\left(\frac{\pi}{2}\right)^{2}\left(\frac{1}{6}+\frac{1}{3 n^{2}}\right)(z-n i)^{2}+\ldots\right]} \\
= & \frac{8 n^{2}}{\pi^{3}(z-n i)^{3}}(-1)^{\frac{n}{2}+1} e^{-n x} \times \\
& {\left[1+i x(z-n i)-\frac{1}{2} x^{2}(z-n i)^{2}+\ldots\right]\left[1-\left(\frac{\pi}{2}\right)^{2}\left(\frac{1}{6}+\frac{1}{3 n^{2}}\right)(z-n i)^{2}+\ldots\right] }
\end{aligned}
$$

The residue of $f(z)$ at the double pole $z=n i$ is equal to the coefficient of $(z-n i)^{-1}$ in the Laurent expansion. Consequently,

$$
\begin{aligned}
\operatorname{Res}(f, n i) & =\frac{8 n^{2}}{\pi^{3}}(-1)^{\frac{n}{2}}\left[\frac{1}{2} x^{2}+\left(\frac{\pi}{2}\right)^{2}\left(\frac{1}{6}+\frac{1}{3 n^{2}}\right)\right] e^{-n x} \\
& =\frac{4}{\pi^{3}}(-1)^{\frac{n}{2}}\left[(n x)^{2}+\frac{\pi^{2}}{12}\left(n^{2}+2\right)\right] e^{-n x}
\end{aligned}
$$

Note that

$$
\begin{aligned}
& \lim _{\epsilon_{1} \rightarrow 0} \int_{\gamma_{\epsilon_{1}}} f(z) d z=i \pi \operatorname{Res}\{f(z) ; 0\}=2 i \\
& \lim _{\epsilon_{2} \rightarrow 0} \int_{\gamma_{\epsilon_{2}}} f(z) d z=i \pi \operatorname{Res}\{f(z) ; 2 n i\}=2 i e^{-2 n x}
\end{aligned}
$$

I now use Cauchy's residue theorem, with $R, S \rightarrow \infty$ independently and 
$\epsilon_{1}, \epsilon_{2} \rightarrow 0$. Using (4.2.23), I find that

$$
\begin{aligned}
& \int_{-\infty}^{\infty} \frac{e^{i p x}}{\cosh ^{2}\left(\frac{\pi}{2 n} p\right) \sinh \left(\frac{\pi}{2} p\right)} d p-\int_{-\infty}^{\infty} \frac{e^{i x(p+2 n i)}}{\cosh ^{2}\left(\frac{\pi}{2 n}(p+2 n i)\right) \sinh \left(\frac{\pi}{2}(p+2 n i)\right)} d p \\
= & {\left[1-e^{-2 n x}\right] \int_{-\infty}^{\infty} \frac{e^{i p x}}{\cosh ^{2}\left(\frac{\pi}{2 n} p\right) \sinh \left(\frac{\pi}{2} p\right)} d p } \\
= & 2 \pi i\left[\frac{1}{\pi}+\frac{1}{\pi} e^{-2 n x}+\frac{2}{\pi} \sum_{\substack{k=1 \\
k \neq \frac{n}{2}}}^{n-1}(-1)^{k} \frac{e^{-2 k x}}{\cos ^{2}\left(\frac{k}{n} \pi\right)}+\frac{4}{\pi^{3}}(-1)^{\frac{n}{2}}\left[(n x)^{2}+\frac{\pi^{2}}{12}\left(n^{2}+2\right)\right] e^{-n x}\right] \\
\therefore & \int_{-\infty}^{\infty} \frac{e^{i p x}}{\cosh ^{2}\left(\frac{\pi}{2 n} p\right) \sinh \left(\frac{\pi}{2} p\right)} d p \\
= & i \operatorname{cosech}(n x)\left(e^{n x}+e^{-n x}+2 \sum_{\substack{k=1 \\
k \neq \frac{n}{2}}}^{n-1}(-1)^{k} \frac{e^{-(2 k-n) x}}{\cos ^{2}\left(\frac{k}{n} \pi\right)}+\frac{4}{\pi^{2}}(-1)^{\frac{n}{2}}\left[(n x)^{2}+\frac{\pi^{2}}{12}\left(n^{2}+2\right)\right]\right) \\
= & 2 i \operatorname{cosech}(n x)\left(\frac{2}{\pi^{2}}(-1)^{\frac{n}{2}}\left[(n x)^{2}+\frac{\pi^{2}}{12}\left(n^{2}+2\right)\right]+\cosh (n x)\right. \\
& \left.+2 \sum_{\substack{k=1 \\
n \geq 4}}^{\frac{n}{2}-1} \frac{(-1)^{k}}{\cos ^{2}\left(\frac{k}{n} \pi\right)} \cosh ([n-2 k] x)\right) .
\end{aligned}
$$

Using equations (6.1.17) and (6.1.21), I finally obtain

$$
\begin{aligned}
& \operatorname{sech}(n x) \star \operatorname{sech}(n x) \star[1+\tanh (x)] \\
& =\left(\frac{\pi}{n}\right)^{2}\left\{1+\operatorname{cosech}(n x)\left(\frac{2}{\pi^{2}}(-1)^{\frac{n}{2}}\left[(n x)^{2}+\frac{\pi^{2}}{12}\left(n^{2}+2\right)\right]\right.\right. \\
& \left.\left.+\cosh (n x)+2 \sum_{\substack{k=1 \\
n \geq 4}}^{\frac{n}{2}-1} \frac{(-1)^{k}}{\cos ^{2}\left(\frac{k}{n} \pi\right)} \cosh ([n-2 k] x)\right)\right\}
\end{aligned}
$$

The results for $n=2(j=1)$ and $n=4(j=2)$ are displayed in Table 6.3.

Also, the result for $n=2$ is plotted in Fig. 5.4 (in red). 
Case (ii): $\quad \sigma=\frac{1}{r}, \quad r=2 m+1, \quad m \in \mathbb{N}$

The integral I now have to calculate is

$$
\int_{-\infty}^{\infty} \frac{e^{i p x}}{\cosh ^{2}\left(\frac{\pi}{2 r} p\right) \sinh \left(\frac{\pi}{2} p\right)} d p
$$

For this case I use the same indented contour as shown in Fig. 4.1, except for the height of the contour is now $2 r i$. Let

$$
f(z)=\frac{e^{i z x}}{\cosh ^{2}\left(\frac{\pi}{2 r} z\right) \sinh \left(\frac{\pi}{2} z\right)} .
$$

$f(z)$ has $\mathrm{r}+1$ simple poles at $z=2 m i(m \in \mathbb{N}, m \leq r)$ which have the same residue as shown in (6.1.25) ( $n$ exchanged with $r$ ), and one double pole at $z=r i$. Note that

$$
\begin{aligned}
& \lim _{\epsilon_{1} \rightarrow 0} \int_{\gamma_{\epsilon_{1}}} f(z) d z=i \pi \operatorname{Res}\{f(z) ; 0\}=2 i, \\
& \lim _{\epsilon_{2} \rightarrow 0} \int_{\gamma_{\epsilon_{2}}} f(z) d z=i \pi \operatorname{Res}\{f(z) ; 2 n i\}=-2 i e^{-2 n x} .
\end{aligned}
$$

In order to calculate the residue at the double pole, I consider a Laurent expansion of $f(z)$ at $z=r i$. To enable me to do this, I require the Taylor series expansion of $\sinh \left(\frac{\pi}{2} z\right)$ at $z=r i$, which is given by

$$
\begin{aligned}
& \sinh \left(\frac{\pi}{2} r i\right)+\frac{\pi}{2} \cosh \left(\frac{\pi}{2} r i\right)(z-r i)+\frac{1}{2}\left(\frac{\pi}{2}\right)^{2} \sinh \left(\frac{\pi}{2} r i\right)(z-r i)^{2}+\ldots \\
= & (-1)^{\frac{r-1}{2}} i\left[1+\frac{1}{2}\left(\frac{\pi}{2}\right)^{2}(z-r i)^{2}+\ldots\right]
\end{aligned}
$$

Using equations (6.1.36), (4.2.14) and (4.2.15), I find that the Laurent expansion of $f(z)$ at $z=r i$ is given by

$$
\frac{4 r^{2}}{\pi^{2}}(-1)^{\frac{r-1}{2}} i \frac{e^{-r x}[1+i x(z-r i)+\ldots]}{(z-r i)^{2}} .
$$

Consequently,

$$
\operatorname{Res}\{f, r i\}=\left(\frac{2 r}{\pi}\right)^{2}(-1)^{\frac{r+1}{2}} x e^{-r x}
$$


Using Cauchy's residue, with $R, S \rightarrow \infty$ independently and $\epsilon_{1}, \epsilon_{2} \rightarrow 0$, I find that

$$
\begin{aligned}
& \int_{-\infty}^{\infty} \frac{e^{i p x}}{\cosh ^{2}\left(\frac{\pi}{2 r} p\right) \sinh \left(\frac{\pi}{2} p\right)} d p-\int_{-\infty}^{\infty} \frac{e^{i x(p+2 r i)}}{\cosh ^{2}\left(\frac{\pi}{2 r}(p+2 r i)\right) \sinh \left(\frac{\pi}{2}(p+2 r i)\right)} d p \\
= & {\left[1+e^{-2 r x}\right] \int_{-\infty}^{\infty} \frac{e^{i p x}}{\cosh ^{2}\left(\frac{\pi}{2 r} p\right) \sinh \left(\frac{\pi}{2} p\right)} d p } \\
= & 2 \pi i\left[\frac{1}{\pi}-\frac{1}{\pi} e^{-2 r x}+\frac{2}{\pi} \sum_{k=1}^{r-1}(-1)^{k} \frac{e^{-2 k x}}{\cos ^{2}\left(\frac{k}{r} \pi\right)}+\left(\frac{2 r}{\pi}\right)^{2}(-1)^{\frac{r+1}{2}} x e^{-r x}\right] \\
\therefore & \int_{-\infty}^{\infty} \frac{e^{i p x}}{\cosh ^{2}\left(\frac{\pi}{2 r} p\right) \sinh \left(\frac{\pi}{2} p\right)} d p \\
= & i \operatorname{sech}(r x)\left(e^{r x}+e^{-r x}+2 \sum_{k=1}^{r-1}(-1)^{k} \frac{e^{-(2 k-r) x}}{\cos ^{2}\left(\frac{k}{r} \pi\right)}+\frac{4 r^{2}}{\pi}(-1)^{\frac{r+1}{2}} x\right) \\
= & 2 i \operatorname{sech}(r x)\left(\frac{2 r^{2}}{\pi}(-1)^{\frac{r+1}{2}} x+\sinh (r x)+2 \sum_{k=1}^{\frac{r-1}{2}} \frac{(-1)^{k}}{\cos ^{2}\left(\frac{k}{r} \pi\right)} \sinh ([r-2 k] x)\right) .
\end{aligned}
$$

Using equations (6.1.17) and (6.1.21), I obtain

$$
\begin{aligned}
& \operatorname{sech}(r x) \star \operatorname{sech}(r x) \star[1+\tanh (x)] \\
& =\left(\frac{\pi}{r}\right)^{2}\left\{1+\operatorname{sech}(r x)\left(\frac{2 r^{2}}{\pi}(-1)^{\frac{r+1}{2}} x+\sinh (r x)\right.\right. \\
& \left.\left.+2 \sum_{\substack{k=1 \\
r \geq 3}}^{\frac{r-1}{2}} \frac{(-1)^{k}}{\cos ^{2}\left(\frac{k}{r} \pi\right)} \sinh ([r-2 k] x)\right)\right\} .
\end{aligned}
$$

The result for $r=1(j=0)$ is given below in Table 6.3.

\section{Scaling function convolutions for $G^{\prime \prime}$}

To develop the part of the model for $g_{2}(x)$ emanating from the scaling function part of $h$, I require the following convolutions for different values of 


\begin{tabular}{|c|c|}
\hline sampling rate $(j)$ & $\phi_{j, 0}^{\star}(x) \star[1+\tanh (x)]$ \\
\hline 0 & $\pi^{2}\left(1+\operatorname{sech}(x)\left[\sinh (x)-\frac{2}{\pi} x\right]\right)$ \\
\hline 1 & $\frac{1}{4}\left[\pi^{2}+\operatorname{cosech}(2 x)\left(\pi^{2}[\cosh (2 x)-1]-8 x^{2}\right)\right]$ \\
\hline 2 & $\frac{1}{16}\left[\pi^{2}+\operatorname{cosech}(4 x)\left(\pi^{2}[\cosh (4 x)-4 \cosh (2 x)+3]+32 x^{2}\right)\right]$ \\
\hline
\end{tabular}

Table 6.3: Scaling function convolutions for fitting $G^{\prime}$ data.

$\sigma:$

$$
\operatorname{sech}\left(\frac{x}{\sigma}\right) \star \operatorname{sech}\left(\frac{x}{\sigma}\right) \star \operatorname{sech}(x) .
$$

By taking the Fourier transform of (6.1.41), and using the convolution theorem, I find that

$$
\left[\operatorname{sech}\left(\frac{x}{\sigma}\right) \star \operatorname{sech}\left(\frac{x}{\sigma}\right) \star \operatorname{sech}(x)\right]^{\wedge}(p)=\sigma^{2} \pi^{3} \operatorname{sech}^{2}\left(\frac{\sigma \pi}{2} p\right) \operatorname{sech}\left(\frac{\pi}{2} p\right) .
$$

By taking the inverse Fourier transform I obtain

$$
\operatorname{sech}\left(\frac{x}{\sigma}\right) \star \operatorname{sech}\left(\frac{x}{\sigma}\right) \star \operatorname{sech}(x)=\frac{(\sigma \pi)^{2}}{2} \int_{-\infty}^{\infty} \frac{e^{i p x}}{\cosh ^{2}\left(\frac{\sigma \pi}{2} p\right) \cosh \left(\frac{\pi}{2} p\right)} d p .
$$

I shall now calculate this integral for different values of $\sigma$.

Case (i): $\quad \sigma=\frac{1}{n}, \quad n=2 m, \quad m \in \mathbb{N}^{+}$

I use the contour displayed in Fig 4.2. Let

$$
f(z)=\frac{e^{i z x}}{\cosh ^{2}\left(\frac{\pi}{2 n} z\right) \cosh \left(\frac{\pi}{2} z\right)} .
$$

Within the contour $\gamma, f(z)$ has $n$ simple poles at $z=(2 m+1) i(m \in \mathbb{N}$, $m \leq n-1)$ and a double pole at $z=n i$. Let $f(z)=\frac{g(z)}{h(z)}$, where

$$
\begin{aligned}
& g(z)=\frac{e^{i z x}}{\cosh ^{2}\left(\frac{\pi}{2 n} z\right)} \\
& h(z)=\cosh \left(\frac{\pi}{2} z\right) .
\end{aligned}
$$


Then

$$
\begin{aligned}
\operatorname{Res}\{f(z) ;(2 m+1) i\} & =\frac{g[(2 m+1) i]}{h^{\prime}[(2 m+1) i]} \\
& =\frac{2}{\pi} \frac{e^{-(2 m+1) x}}{\cosh ^{2}\left(\frac{2 m+1}{2 n} \pi i\right) \sinh \left(\frac{2 m+1}{2} \pi i\right)} \\
& =\frac{2}{\pi}(-1)^{m+1} i \frac{e^{-(2 m+1) x}}{\cos ^{2}\left(\frac{2 m+1}{2 n} \pi\right)}
\end{aligned}
$$

In order to calculate the residue at the double pole, I consider a Laurent expansion of $f(z)$ at $z=n i$. Using equations (4.2.14), (4.2.15) and (4.2.86), I find that this expansion is of the form

$$
\left(\frac{2 n}{\pi}\right)^{2}(-1)^{\frac{n}{2}+1} \frac{e^{-n x}[1+i x(z-n i)+\ldots]}{(z-n i)^{2}}
$$

Consequently,

$$
\operatorname{Res}\{f, n i\}=\left(\frac{2 n}{\pi}\right)^{2}(-1)^{\frac{n}{2}+1} i x e^{-n x}
$$

By Cauchy's residue theorem, as $R, S \rightarrow \infty$ independently, I obtain

$$
\begin{aligned}
& \int_{-\infty}^{\infty} \frac{e^{i p x}}{\cosh ^{2}\left(\frac{\pi}{2 n} p\right) \cosh \left(\frac{\pi}{2} p\right)} d p-\int_{-\infty}^{\infty} \frac{e^{i x(p+2 n i)}}{\cosh ^{2}\left(\frac{\pi}{2 n}(p+2 n i)\right) \cosh \left(\frac{\pi}{2}(p+2 n i)\right)} d p \\
= & {\left[1-e^{-2 n x}\right] \int_{-\infty}^{\infty} \frac{e^{i p x}}{\cosh ^{2}\left(\frac{\pi}{2 n} p\right) \cosh \left(\frac{\pi}{2} p\right)} d p } \\
= & 2 \pi i\left[\frac{2}{\pi} i \sum_{k=0}^{n-1}(-1)^{k+1} \frac{e^{-(2 k+1) x}}{\cos ^{2}\left(\frac{2 k+1}{2 n} \pi\right)}+\left(\frac{2 n}{\pi}\right)^{2}(-1)^{\frac{n}{2}+1} i x e^{-n x}\right] \\
\therefore & \int_{-\infty}^{\infty} \frac{e^{i p x}}{\cosh ^{2}\left(\frac{\pi}{2 n} p\right) \cosh \left(\frac{\pi}{2} p\right)} d p=2 \operatorname{cosech}(n x)\left[\sum_{k=0}^{n-1}(-1)^{k} \frac{e^{[n-(2 k+1)] x}}{\cos ^{2}\left(\frac{2 k+1}{2 n} \pi\right)}+\frac{2 n^{2}}{\pi}(-1)^{\frac{n}{2}} x\right] \\
= & 4 \operatorname{cosech}(n x)\left[\frac{n^{2}}{\pi}(-1)^{\frac{n}{2}} x+\sum_{\substack{k=0 \\
n \geq 2}}^{k=\frac{n-2}{2}} \frac{(-1)^{k}}{\cos \left(\frac{2 k+1}{2 n} \pi\right)} \sinh ([n-(2 k+1)] x)\right] .
\end{aligned}
$$


Consequently, using equation (6.1.43), I obtain

$$
\begin{aligned}
& \operatorname{sech}(n x) \star \operatorname{sech}(n x) \star \operatorname{sech}(x) \\
= & 2\left(\frac{\pi}{n}\right)^{2} \operatorname{cosech}(n x)\left[\frac{n^{2}}{\pi}(-1)^{\frac{n}{2}} x+\sum_{\substack{k=0 \\
n \geq 2}}^{k=\frac{n-2}{2}} \frac{(-1)^{k}}{\cos \left(\frac{2 k+1}{2 n} \pi\right)} \sinh ([n-(2 k+1)] x)\right] .
\end{aligned}
$$

The results for $n=2(j=1)$ and $n=4(j=2)$ are displayed in Table 6.4. Also, the result for $n=2$ is plotted in Fig. 6.4 (in blue).

Case (ii): $\quad \sigma=\frac{1}{r}, \quad r=2 m+1, \quad m \in \mathbb{N}$

The integral I now have to calculate is

$$
\int_{-\infty}^{\infty} \frac{e^{i p x}}{\cosh ^{2}\left(\frac{\pi}{2 r} p\right) \cosh \left(\frac{\pi}{2} p\right)} d p
$$

For this case I use the same contour as shown in Fig. 4.2, except for the height of the contour is now $2 r i$. Let

$$
f(z)=\frac{e^{i z x}}{\cosh ^{2}\left(\frac{\pi}{2 r} z\right) \cosh \left(\frac{\pi}{2} z\right)} .
$$

$f(z)$ has $\mathrm{r}-1$ simple poles at $z=(2 m+1) i\left(m \in \mathbb{N} \backslash\left\{\frac{r-1}{2}\right\}, m \leq r-1\right)$ which have the same residue as shown in (6.1.47) ( $n$ exchanged with $r$ ), and one triple pole at $z=r i$. To calculate the residue of the pole at $z=r i, \mathrm{I}$ construct a Laurent expansion of $f(z)$ at $z=r i$. Using equations (4.2.14), (4.2.15) and (4.2.58), I obtain the following Laurent expansion of $f(z)$ at $z=r i$

$$
\frac{4 r^{2}(-1)^{\frac{r-1}{2}} i e^{-r x}}{\pi^{3}(z-r i)^{3}}\left[1+i x(z-r i)-(z-r i)^{2}\left\{x^{2}+\frac{\pi^{2}}{12 r^{2}}\left(r^{2}+2\right)\right\}+\ldots\right]
$$

Consequently,

$$
\operatorname{Res}(f, r i)=\frac{4(-1)^{\frac{r+1}{2}} i e^{-r x}}{\pi^{3}}\left[(r x)^{2}+\frac{\pi^{2}}{12}\left(r^{2}+2\right)\right] .
$$


By Cauchy's residue theorem, as $R, S \rightarrow \infty$ independently, I obtain

$$
\begin{aligned}
& \int_{-\infty}^{\infty} \frac{e^{i p x}}{\cosh ^{2}\left(\frac{\pi}{2 r} p\right) \cosh \left(\frac{\pi}{2} p\right)} d p-\int_{-\infty}^{\infty} \frac{e^{i x(p+2 r i)}}{\cosh ^{2}\left(\frac{\pi}{2 r}(p+2 r i)\right) \cosh \left(\frac{\pi}{2}(p+2 r i)\right)} d p \\
= & {\left[1+e^{-2 r x}\right] \int_{-\infty}^{\infty} \frac{e^{i p x}}{\cosh ^{2}\left(\frac{\pi}{2 r} p\right) \cosh \left(\frac{\pi}{2} p\right)} d p } \\
= & 2 \pi i\left[\frac{4(-1)^{\frac{r+1}{2}} i e^{-r x}}{\pi^{3}}\left[(r x)^{2}+\frac{\pi^{2}}{12}\left(r^{2}+2\right)\right]+\frac{2}{\pi} i \sum_{\substack{k=0 \\
k \neq \frac{r-1}{2}}}^{r-1}(-1)^{k+1} \frac{e^{-(2 k+1) x}}{\cos ^{2}\left(\frac{2 k+1}{2 r} \pi\right)}\right] \\
\therefore & \int_{-\infty}^{\infty} \frac{e^{i p x}}{\cosh ^{2}\left(\frac{\pi}{2 r} p\right) \cosh \left(\frac{\pi}{2} p\right)} d p \\
= & 2 \operatorname{sech}(r x)\left[\frac{2(-1)^{\frac{r-1}{2}}}{\pi^{2}}\left[(r x)^{2}+\frac{\pi^{2}}{12}\left(r^{2}+2\right)\right]+\sum_{\substack{k=0 \\
k \neq \frac{r-1}{2}}}^{r-1}(-1)^{k} \frac{e^{[r-(2 k+1)] x}}{\cos ^{2}\left(\frac{2 k+1}{2 r} \pi\right)}\right] \\
= & 4 \operatorname{sech}(r x)\left[\frac{(-1)^{\frac{r-1}{2}}}{\pi^{2}}\left[(r x)^{2}+\frac{\pi^{2}}{12}\left(r^{2}+2\right)\right]+\sum_{\substack{k=\frac{r-3}{2} \\
r=0 \\
r \geq 3}}^{r(-1)^{k}} \frac{\cosh ^{2}([r-(2 k+1)] x)}{\cos ^{2}\left(\frac{2 k+1}{2 r} \pi\right)}\right] .
\end{aligned}
$$

Consequently, using equation (6.1.43), I obtain

$$
\begin{aligned}
& \operatorname{sech}(r x) \star \operatorname{sech}(r x) \star \operatorname{sech}(x) \\
& =2\left(\frac{\pi}{r}\right)^{2} \operatorname{sech}(r x)\left[\frac{(-1)^{\frac{r-1}{2}}}{\pi^{2}}\left[(r x)^{2}+\frac{\pi^{2}}{12}\left(r^{2}+2\right)\right]\right. \\
& \left.+\sum_{\substack{k=0 \\
r \geq 3}}^{k=\frac{r-3}{2}}(-1)^{k} \frac{\cosh ([r-(2 k+1)] x)}{\cos ^{2}\left(\frac{2 k+1}{2 r} \pi\right)}\right] .
\end{aligned}
$$

The result for $r=1(j=0)$ is displayed below in Table 6.4. I have not calculated the mother wavelet convolutions for $G^{\prime}$ and $G^{\prime \prime}$, because in this 


\begin{tabular}{|c|c|c|c|}
\hline sampling rate $(j)$ & \multicolumn{3}{|c|}{$\phi_{j, 0}^{\star}(x) \star \operatorname{sech}(x)$} \\
\hline 0 & \multicolumn{3}{|c|}{$2\left(x^{2}+\frac{\pi^{2}}{4}\right) \operatorname{sech}(x)$} \\
\hline 1 & \multicolumn{3}{|c|}{$\pi \operatorname{cosech}(2 x)[\pi \sinh (x)-2 x]$} \\
\hline 2 & $\frac{\pi}{2} \operatorname{cosech}(4 x)$ & $\pi\left(\frac{1}{2+\sqrt{2}} \sinh (3 x)-\frac{1}{2-\sqrt{2}} \sinh (x)\right)$ & $+4 x$ \\
\hline
\end{tabular}

Table 6.4: Scaling function convolutions for fitting $G^{\prime \prime}$ data.

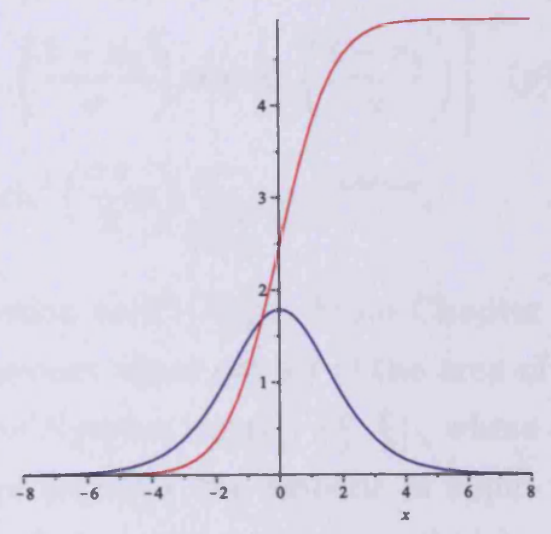

Figure 6.4: $\phi_{1,0}^{\star}(x) \star[1+\tanh (x)]($ red $), \phi_{1,0}^{\star}(x) \star \operatorname{sech}(x)$ (blue).

thesis I have only used the models in this section to fit real data. So, for the same reason as stated in Chapter 5 , we disregard the mother wavelet part oh $h$ when fitting real data. 


\subsubsection{Results for real data}

As in Chapter 5, I ignore the mother wavelet part of $h$ and consider $m$ sparse approximations for $h$ over an appropriate index set $\Lambda$ with $m$ elements:

$$
\begin{aligned}
h(t) & =\sum_{k \in \Lambda_{m}} c_{k} \Gamma\left(\frac{t-x_{k}}{\sigma}\right), \quad\left\|\mathbf{b}_{0}\right\|=m, \\
\text { where } \Gamma(t) & =2 t \operatorname{cosech}(t) \\
\text { and } x_{k} & =x_{0}+k a .
\end{aligned}
$$

From the formulation of $h$ above, and using (6.1.3) and the scaling and translation properties of the Fourier transform (see Table 1.1), I obtain

$$
\begin{aligned}
\widehat{h}(p) & =\left[\sum_{k \in \Lambda_{m}} c_{k}\left(\frac{t-x_{k}}{\sigma}\right) \operatorname{cosech}\left(\frac{t-x_{k}}{\sigma}\right)\right]^{\wedge}(p) \\
& =\frac{(\sigma \pi)^{2}}{2} \operatorname{sech}^{2}\left(\frac{\sigma \pi}{2} p\right) \sum_{k \in \Lambda_{m}} c_{k} e^{-i 2 \sigma x_{k} p},
\end{aligned}
$$

which is windowed by the function $\operatorname{sech}^{2}\left(\frac{\sigma \pi}{2} p\right)$. From Chapter 5 , it was explained that super-resolution occurs when not all of the area of this window function is contained within the Nyquist range $\left[-\frac{\pi}{s}, \frac{\pi}{s}\right]$, where $s$ is the sampling rate of the data. I again consider the amount of super-resolution to be the percentage of the area of the window function that lies outside this range. Then the amount of super-resolution for a particular value of $\sigma$ can be calculated as follows. Applying property (1.5.3) to equation (2.1.22), it can be shown that

$$
\frac{\sigma \pi}{4} \int_{-\infty}^{\infty} \operatorname{sech}^{2}\left(\frac{\sigma \pi}{2} p\right) d p=1
$$

I can then express the percentage of super-resolution, $S R$, for a particular value of $\sigma$ as

$$
S R=\left[1-\frac{\sigma \pi}{4} \int_{-\frac{\pi}{s}}^{\frac{\pi}{s}} \operatorname{sech}^{2}\left(\frac{\sigma \pi}{2} p\right) d p\right] \times 100 \%
$$


I use the search algorithm introduced in Section 5.4 to compare the results from the original sech dictionary and the new $x$ cosech dictionary when fitting PB1 data. For consistency, I keep the value of the sparsity at $m=4$.

The spectrum is of the form

$$
h(t)=\sum_{k=0}^{3} c_{k}\left(x-x_{k}\right) \operatorname{cosech}\left(\frac{x-x_{k}}{\sigma}\right) .
$$

For this $m$-sparse approximation, I find that the largest value of $\sigma$ that gives an acceptable fit to the storage and loss moduli data is achieved when $\sigma=\frac{1}{4}$, where I have an RMS error of $1.80 \%$ and I obtain a physically acceptable bimodal spectrum (Fig. 6.5(b)). This value of $\sigma$ is smaller than when using the sech dictionary, but this is not surprising, considering that the new basis functions $x \operatorname{cosech}\left(\frac{x}{\sigma}\right)$ are wider than the sech dictionary basis functions $\operatorname{sech}\left(\frac{x}{\sigma}\right)$ at the same value of $\sigma$. Indeed, when $\sigma=\frac{1}{2}$ in the new dictionary, there is a much poorer fit to the data of $3.19 \%$, leading to a bimodal relaxation spectrum (Fig. 6.5(a)) with a more significant negative lobe than the sech dictionary with $\sigma=\frac{1}{2}$ (Fig. 5.2(a)). When $\sigma=\frac{1}{5}$ I obtain a trimodal spectrum (Fig. 6.5(c)). When $\sigma$ is small enough, e.g. $\sigma=\frac{1}{8}$ (Fig. $6.5(\mathrm{~d}))$ I obtain an $m$-mode distribution, giving an excellent fit to the data of $1.24 \%$. The parameter values for $x_{0}$ and $a$, as well as the coefficients $c_{k}$, are displayed in Table 6.5 for each value of $\sigma$.

When $\sigma=\frac{1}{8} \mathrm{I}$ obtain an excellent fit to the data, but for the same reason as discussed when exploring the sech dictionary, I cannot justify that the underlying data supports a four mode distribution. Using my philosophy of choosing the smallest number of peaks that gives an acceptable fit to the data, I have concluded from the results of this dictionary that this particular polymer is most likely to have a bimodal relaxation spectrum, which is consistent with the results obtained from the sech dictionary.

To select an optimal value for $\sigma$ I constructed a homotopic approximation for the spectra and data fits, linearly interpolating between the values of 


\begin{tabular}{|c|c|c|c|c|c|c|c|}
\hline$\sigma$ & $x_{0}$ & $a$ & $c_{0}$ & $c_{1}$ & $c_{2}$ & $c_{3}$ & RMS \\
\hline $1 / 2$ & 4.76 & 0.10 & -20.7 & 64.6 & -67.2 & 23.3 & 3.19 \\
\hline $1 / 4$ & 2.65 & 1.40 & -0.0204 & 1.13 & 0.972 & 0.704 & 1.80 \\
\hline $1 / 5$ & 2.29 & 1.67 & -0.0620 & 1.58 & 6.07 & 0.901 & 1.46 \\
\hline $1 / 6$ & 3.92 & 1.81 & 2.21 & 1.11 & 1.06 & 0.675 & 1.30 \\
\hline $1 / 8$ & 3.79 & 1.32 & 3.32 & 1.71 & 1.26 & 1.82 & 1.24 \\
\hline
\end{tabular}

Table 6.5: Optimal parameter values for varying $\sigma(m=4)$. The $c_{k}$ should be multiplied by $10^{6}$ to attain their true value.

$\sigma=\frac{1}{4}$ (where the spectrum is bimodal) and $\sigma=\frac{1}{5}$ (where there are three peaks). I calculated the total curvature, $T$, for each spectrum in this range (decreasing $\sigma$ in steps of 0.01 from $\frac{1}{4}$ ). I get minimum total curvature when $\sigma=\frac{1}{4}$. Consequently, I believe that the optimal amount of resolution for this approximation, using merely four basis elements, is $\sigma=\frac{1}{4}$, which gives an RMS of $1.80 \%$ and a physically respectable bimodal relaxation spectrum (Fig. 6.5(b)).

Since the sampling rate of the data is approximately 0.38 , the percentage of super-resolution for this optimal value of $\sigma$ is much less than $1 \%$, calculated from equation (6.1.63) as follows

$$
S R=\left[1-\frac{\pi}{16} \int_{-8.24}^{8.24} \operatorname{sech}^{2}\left(\frac{\pi}{8} p\right) d p\right] \times 100 \% \approx 0.31 \%
$$

The results for the amount of super-resolution for this, and other, selected values of $\sigma$ are given in Table 6.6. For different values of $\sigma$, an illustration of how much super-resolution is taking place is given in Fig. 6.6, where in each case I have plotted $\widehat{h}(p)$ against its trigonometric polynomial and the Nyquist bandwidth of the data. As in Chapter 5, these graphs show that if $\sigma$ is too small then the effective bandwidth of the spectrum is wider than the Nyquist bandwidth of the data, resulting in significant super-resolution. 
In Fig. 6.6(a), the maximum amplitude of the trigonometric polynomial is approximately $2 \times 10^{9}$, but I have only shown the part of the function that I can readily compare the effect of the windowing function with other values of $\sigma$ in Figs. 6.6(b)-6.6(d).

In Fig. 6.7(a), I have plotted the respective data fits and relaxation spectra from the two separate dictionaries, using the optimal values of $\sigma$ in each case. These results are very similar, giving consistency in the approximations for the continuous relaxation spectrum of this particular polymer. When I plot the individual basis functions of the two separate dictionaries (normalized so that they have unit area) with the optimal values of $\sigma$ in each case (Fig. 6.7(b)), it becomes clear that I am using very similar basis functions in the two approximations.

\begin{tabular}{|c|c|}
\hline$\sigma$ & Super-resolution (\%) \\
\hline $1 / 2$ & 0.0005 \\
\hline $1 / 4$ & 0.310 \\
\hline $1 / 5$ & 1.12 \\
\hline $1 / 8$ & 7.58 \\
\hline
\end{tabular}

Table 6.6: Percentage of super-resolution for each of the approximations in Fig. 6.5. 

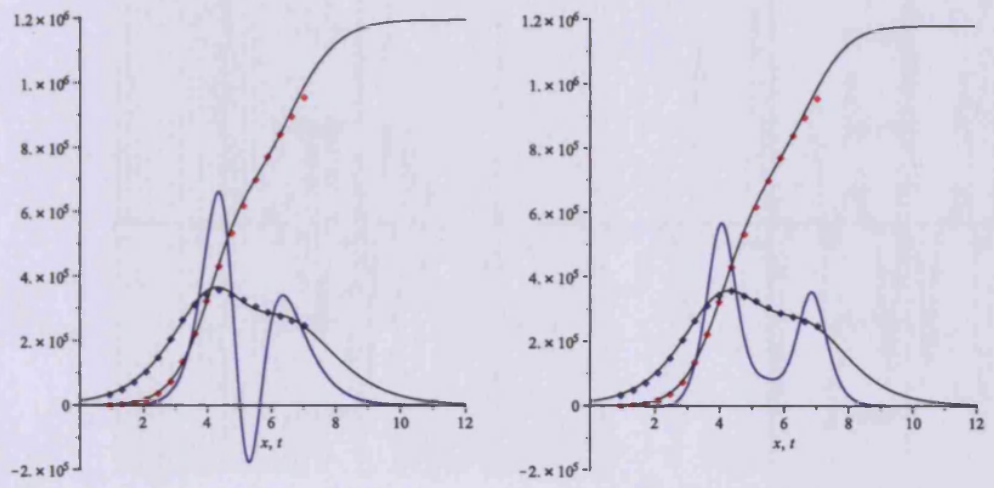

(a) $\sigma=\frac{1}{2}$, RMS error $=3.19 \%$.

(b) $\sigma=\frac{1}{4}$, RMS error $=1.80 \%$.
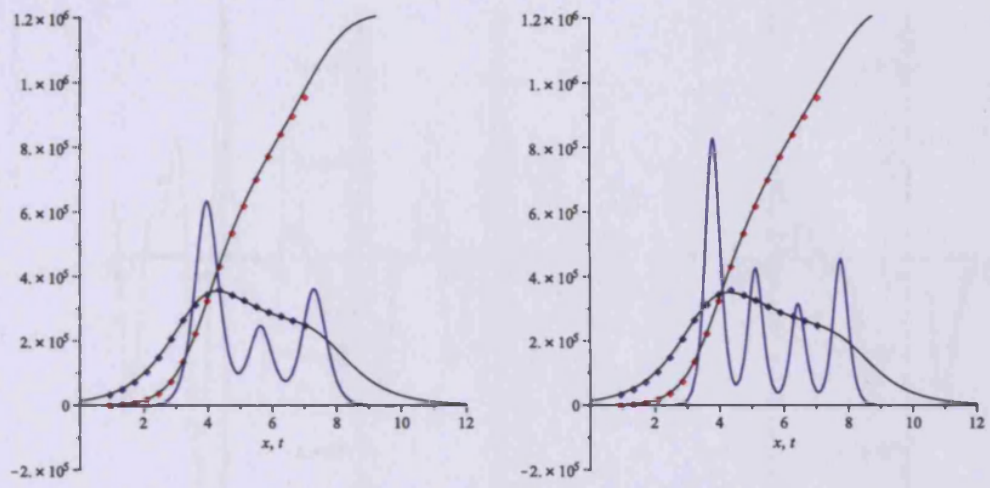

(c) $\sigma=\frac{1}{5}$, RMS error $=1.46 \%$.

(d) $\sigma=\frac{1}{8}$, RMS error $=1.24 \%$.

Figure 6.5: Sparse approximation of PB1 using $x$ cosech dictionary, with $m=4$. 


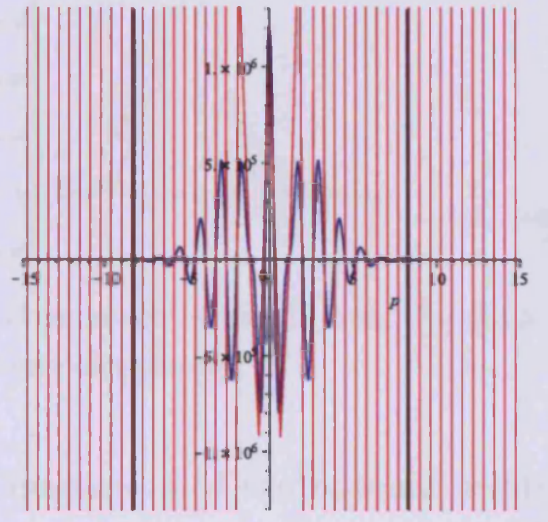

(a) $\sigma=\frac{1}{2}$.

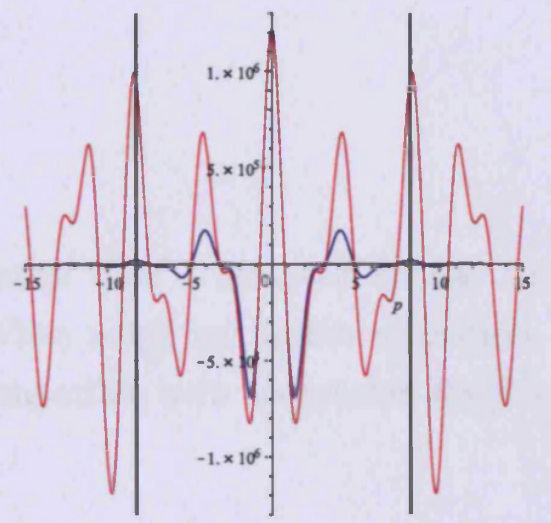

(c) $\sigma=\frac{1}{5}$.

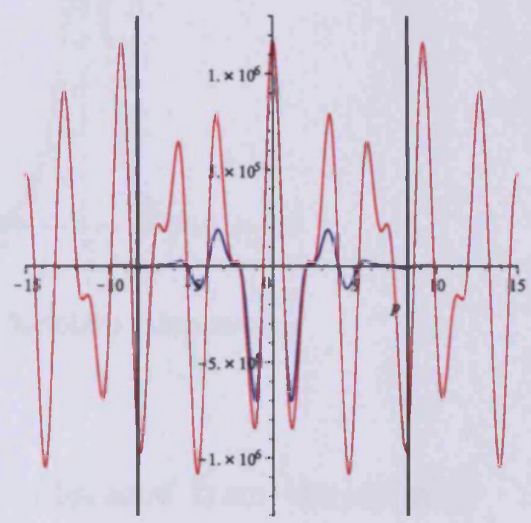

(b) $\sigma=\frac{1}{4}$.

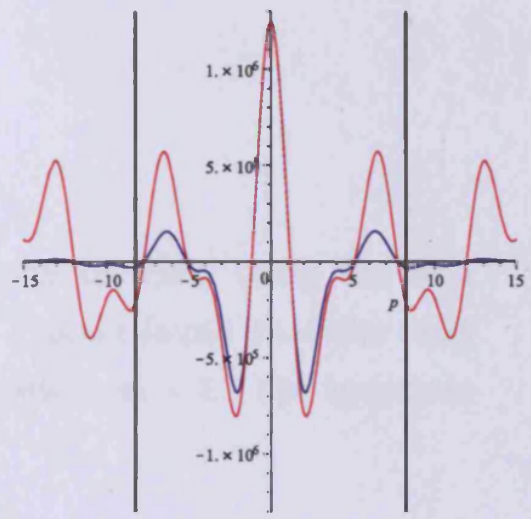

(d) $\sigma=\frac{1}{8}$.

Figure 6.6: Effect of windowing function. Trigonometric polynomial (red), $\widehat{h}(p)$ (blue) and the Nyquist bandwidth of the data (bold black). 

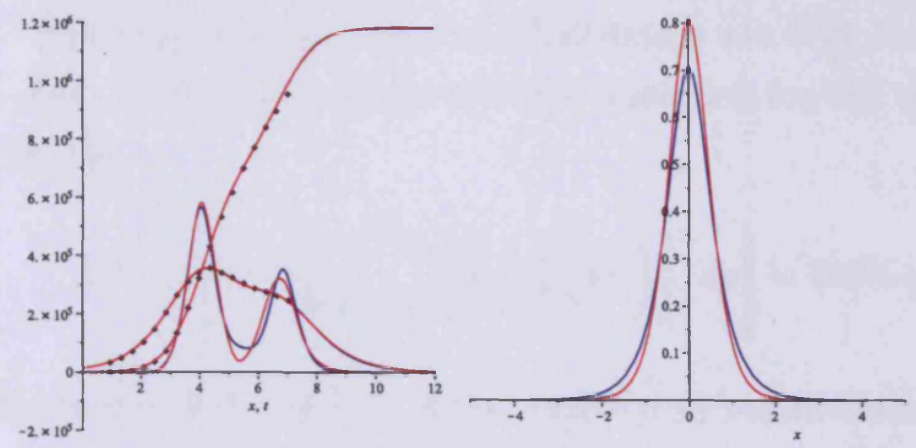

(a) Comparison of results from (b) Basis function comparison. separate dictionaries.

Figure 6.7: Comparison of the optimal results obtained from the $x$ cosech dictionary (blue) and the sech dictionary (red) (a), and of the basis functions used (b).

\section{PB2 data}

For this set of data I achieved similar results to when using the sech dictionary. When using my search algorithm, I again found that the least value of $m$ compatible rxith arpantahlo RMS lovale is $m-5$ The spectrum is of the form $\quad h(t)=\sum_{k=n}^{n} c_{k}\left(x-x_{k}\right) \operatorname{cosech}\left(\frac{x-x_{k}}{\sigma}\right)$.

$$
h(t)=\sum_{k=0}^{4} c_{k}\left(x-x_{k}\right) \operatorname{cosech}\left(\frac{x-x_{k}}{\sigma}\right) .
$$

With $\sigma=\frac{1}{4}$ I get a superb fit to the data, with an RMS error of $1.55 \%$. This leads to a bimodal continuous relaxation spectrum which again has a remarkably similar shape to the optimal spectrum obtained from PB1 data, when approximating $h$ from the $x$ cosech dictionary (Fig. 6.8(a)). This fit is achieved with $x_{0}=-6.99$ and $a=3.40$. 
Since the sampling rate of the PB2 data is $s \approx 0.46$, I use equation (6.1.62) to show that the percentage of super-resolution for this optimal value of $\sigma$ is given by

$$
S R=\left[1-\frac{\pi}{16} \int_{-6.82}^{6.82}\left[\operatorname{sech}\left(\frac{1}{8} \pi p\right)\right]^{2} d p\right] \times 100 \% \approx 0.94 \%
$$

This means that over $99 \%$ of the area of $\widehat{h}(p)$ is contained within the Nyquist range of the data. This is also illustrated in Fig. 6.8(b).

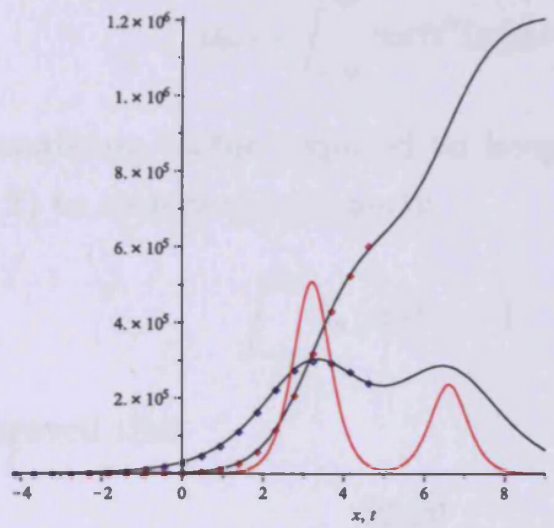

(a) Data fit and spectrum.

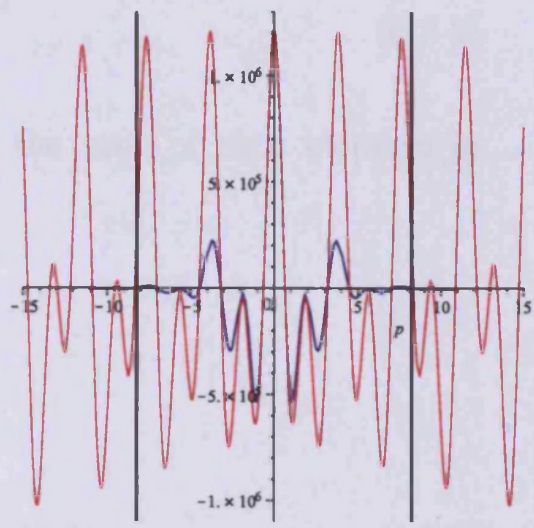

(b) Effect of windowing function.

Figure 6.8: PB2 data results.

\subsection{Error terms in the delta sequence method}

The delta sequence method for deriving successive approximations to the relaxation spectrum is explained by Friedrich [34] and in the $\mathrm{PhD}$ thesis of D. Morgan [58]. This approach generalizes the results obtained by Schwarzl and Staverman [67], and Tschoegl [74]. They introduced an approach to the 
delta function, namely

$$
\delta(x)=\lim _{n \rightarrow \infty} \delta_{n}(x)
$$

with the elements of the delta sequence taking the form

$$
\delta_{n}(x)=\frac{\operatorname{sech}^{n}(x)}{\mu_{n}}
$$

where

$$
\mu_{n}=\int_{-\infty}^{\infty} \operatorname{sech}^{n}(x) d x
$$

$\mu_{n}$ is the normalizing factor required to keep the area of each element in equation (6.2.2) to satisfy the property

$$
\int_{-\infty}^{\infty} \delta_{n}(x) d x=1
$$

Morgan [58] proved that

$$
\begin{aligned}
\mu_{2 n+1} & =\frac{(2 n) !}{2^{2 n}(n !)^{2}} \pi \quad(n \geq 0) \\
\mu_{2 n} & =\frac{2^{2 n}(n !)^{2}}{n(2 n) !} \quad(n \geq 1) .
\end{aligned}
$$

The starting point is the canonical relationships

$$
\begin{aligned}
G^{\prime}(\omega) & =\frac{1}{2} \int_{-\infty}^{\infty} H(\tau)[1+\tanh (\ln (\omega \tau))] d \ln \tau \\
G^{\prime \prime}(\omega) & =\frac{1}{2} \int_{-\infty}^{\infty} H(\tau) \operatorname{sech}(\ln (\omega \tau)) d \ln \tau
\end{aligned}
$$

The first element of the delta sequence is

$$
\delta_{1}(\ln (\omega \tau))=\frac{\operatorname{sech}(\ln (\omega \tau))}{\pi}
$$


which is a symmetric function centred at $\omega \tau=1$. Taking this quantity as a very crude approximation to the delta function,

$$
\begin{aligned}
G^{\prime \prime}(\omega) & =\frac{\pi}{2} \int_{-\infty}^{\infty} H(\tau) \delta_{1}(\ln (\omega \tau)) d \ln \tau \\
& \approx \frac{\pi}{2} \int_{-\infty}^{\infty} H(\tau) \delta(\ln (\omega \tau)) d \ln \tau
\end{aligned}
$$

On writing $H(\tau)=h(\ln \tau)=H\left(e^{\ln \tau}\right)$ and using the sifting property of the delta function, it can be shown that

$$
H(\tau) \approx \frac{2}{\pi} G^{\prime \prime}\left(\tau^{-1}\right)
$$

This first approximation is regarded as the trivial case, as it does not involve any derivatives, and for this purpose, is known as the zeroth approximation of the relaxation spectrum. I have already shown, in Section 3.4, that the error term in this approximation can be derived through the Calderón-Mallat decomposition of the identity, to be

$E(\tau)=\frac{2}{\pi} \int_{-\infty}^{\infty} \int_{0}^{1} H\left(\omega^{-1}\right) \frac{(\omega \tau)^{\frac{1}{s}}}{1+(\omega \tau)^{\frac{2}{s}}}\left[1+\frac{\ln (\omega \tau)}{s}\left\{\frac{1-(\omega \tau)^{\frac{2}{s}}}{1+(\omega \tau)^{\frac{2}{s}}}\right\}\right] \frac{d s}{s^{2}} d l n \omega$.

To proceed along the sequence, higher powers of sech are matched to corresponding logarithmic derivatives of the dynamic moduli; illustrated for the next four cases in equations (6.2.14)-(6.2.17). These take the form of Fredholm integral equations of the first kind.

$$
\begin{aligned}
\frac{1}{2} \int_{-\infty}^{\infty} H(\tau) \operatorname{sech}^{2}(\ln (\omega \tau)) d \ln \tau & =\frac{d G^{\prime}(\omega)}{d \ln \omega} \\
\int_{-\infty}^{\infty} H(\tau) \operatorname{sech}^{3}(\ln (\omega \tau)) d \ln \tau & =G^{\prime \prime}(\omega)-\frac{d^{2} G^{\prime \prime}(\omega)}{d(\ln \omega)^{2}} \\
\int_{-\infty}^{\infty} H(\tau) \operatorname{sech}^{4}(\ln (\omega \tau)) d \ln \tau & =\frac{4}{3} \frac{d G^{\prime}(\omega)}{d \ln \omega}-\frac{1}{3} \frac{d^{3} G^{\prime}(\omega)}{d(\ln \omega)^{3}} \\
\int_{-\infty}^{\infty} H(\tau) \operatorname{sech}^{5}(\ln (\omega \tau)) d \ln \tau & =\frac{3}{4} G^{\prime \prime}(\omega)-\frac{5}{6} \frac{d^{2} G^{\prime \prime}(\omega)}{d(\ln \omega)^{2}}+\frac{1}{12} \frac{d^{4} G^{\prime \prime}(\omega)}{d(\ln \omega)^{4}}
\end{aligned}
$$


The kernels in the above equations, and in the equations that would follow in the delta sequence method, involve powers of the sech function and may also be expressed as

$$
\operatorname{sech}^{n}(\ln (\omega \tau))=\left(\frac{2 \omega \tau}{1+\omega^{2} \tau^{2}}\right)^{n}
$$

showing that as $n$ increases the more impulsive this kernel becomes and hence the more exact the sifting property which inverts the process. Using the powers of sech in the above equations as approximations for the delta function, the following respective approximations for the relaxation spectrum are obtained:

$$
\begin{aligned}
& H_{1}(\tau)=\frac{d G^{\prime}(\omega)}{d \ln \omega} \\
& H_{2}(\tau)=\frac{2}{\pi}\left[G^{\prime \prime}(\omega)-\frac{d^{2} G^{\prime \prime}(\omega)}{d(\ln \omega)^{2}}\right] \\
& H_{3}(\tau)=\frac{3}{4}\left[\frac{4}{3} \frac{d G^{\prime}(\omega)}{d \ln \omega}-\frac{1}{3} \frac{d^{3} G^{\prime}(\omega)}{d(\ln \omega)^{3}}\right] \\
& H_{4}(\tau)=\frac{8}{3 \pi}\left[\frac{3}{4} G^{\prime \prime}(\omega)-\frac{5}{6} \frac{d^{2} G^{\prime \prime}(\omega)}{d(\ln \omega)^{2}}+\frac{1}{12} \frac{d^{4} G^{\prime \prime}(\omega)}{d(\ln \omega)^{4}}\right] .
\end{aligned}
$$

The first three approximations $\left(H_{0}(\tau), H_{1}(\tau)\right.$ and $\left.H_{2}(\tau)\right)$ are classical and appear in the texts of both Ferry [33], Tschoegl [74] and Friedrich [34]. The remaining approximations are far more unfamiliar, mainly due to the fact that higher order numerical differentiation of measured inexact data is necessary.

I will now derive the error term, $E_{n}(\tau)$ in these approximations for the relaxation spectrum, obtained via the delta sequence method. Equations (6.2.19)-(6.2.22) above can be written in the form

$$
H_{n-1}(\tau)=\frac{1}{\mu_{n}} \int_{-\infty}^{\infty} H(\tau) \operatorname{sech}^{n}(\ln (\omega \tau)) d \ln \tau \quad(n \geq 1)
$$


Using the substitutions (2.1.6) and (2.1.7), I obtain

$$
h_{n-1}(t)=\frac{1}{\mu_{n}} h(t) \star \operatorname{sech}^{n}(t) .
$$

Let $\sigma=1$ and choose

$$
\left(\phi_{1} \star \phi_{1}\right)(t)=\operatorname{sech}^{n}(t) .
$$

From (3.1.16) and (6.2.3), I note that

$$
C_{\psi}=\mu_{n}
$$

and hence equation (6.2.24) becomes

$$
h_{n-1}(t)=\frac{1}{C_{\psi}}\left(h \star \phi_{1} \star \phi_{1}\right)(t),
$$

which is the Mallat part of the Calderón-Mallat decomposition of the identity (3.2.10), with $\sigma=1$. Since $h_{n}(t)$ is an approximation for $h(t)$, the error in this approximation is the Calderón part

$$
E_{n}(\tau)=\frac{1}{\mu_{n}} \int_{0}^{1}\left(h \star \psi_{s} \star \psi_{s}\right)(t) \frac{d s}{s^{2}} .
$$

It follows from (3.1.17) and (3.4.9), that

$$
\left(\psi_{s} \star \psi_{s}\right)(t)=\operatorname{sech}^{n}\left(\frac{t}{s}\right)\left[1-n \frac{t}{s} \tanh \left(\frac{t}{s}\right)\right] .
$$

By using (6.2.29) and $\omega=\tau^{-1}$, I can now express the error term as

$$
\begin{aligned}
E_{n}(\tau)= & \frac{1}{\mu_{n}} \int_{0}^{1} h(t) \star \operatorname{sech}^{n}\left(\frac{t}{s}\right)\left[1-n \frac{t}{s} \tanh \left(\frac{t}{s}\right)\right] \frac{d s}{s^{2}} \\
= & \frac{1}{\mu_{n}} \int_{0}^{1} \int_{-\infty}^{\infty} h(x) \operatorname{sech}^{n}\left(\frac{t-x}{s}\right)\left[1-n \frac{t-x}{s} \tanh \left(\frac{t-x}{s}\right)\right] d x \frac{d s}{s^{2}} \\
= & \frac{1}{\mu_{n}} \int_{0}^{1} \int_{-\infty}^{\infty} H\left(\omega^{-1}\right) \operatorname{sech}^{n}\left(\frac{-[\ln \tau+\ln \omega]}{s}\right) \\
& \quad\left[1-n \frac{(-[\ln \tau+\ln \omega])}{s} \tanh \left(\frac{-[\ln \tau+\ln \omega]}{s}\right)\right] d \ln \omega \frac{d s}{s^{2}}
\end{aligned}
$$




$$
\begin{aligned}
=\frac{1}{\mu_{n}} \int_{-\infty}^{\infty} \int_{0}^{1} H\left(\omega^{-1}\right) \operatorname{sech}^{n}\left(\left[\ln (\omega \tau)^{\frac{1}{s}}\right]\right) \\
\\
\quad\left[1+n \ln (\omega \tau)^{\frac{1}{s}} \tanh \left[\ln (\omega \tau)^{-\frac{1}{s}}\right]\right] \frac{d s}{s^{2}} d \ln \omega
\end{aligned}
$$

Substituting (3.4.12) and (3.4.13) into (6.2.30), I can express the error term in the delta sequence approximation in terms of the physical variables $\omega$ and $\tau$ as

$E_{n}(\tau)=\frac{2^{n}}{\mu_{n}} \int_{-\infty}^{\infty} \int_{0}^{1} H\left(\omega^{-1}\right) \frac{(\omega \tau)^{\frac{n}{s}}}{\left[1+(\omega \tau)^{\frac{2}{s}}\right]^{n}}\left[1+\frac{n \ln (\omega \tau)}{s}\left\{\frac{1-(\omega \tau)^{\frac{2}{s}}}{1+(\omega \tau)^{\frac{2}{s}}}\right\}\right] \frac{d s}{s^{2}} d \ln \omega$.

\subsection{Wavelet families in linear viscoelasticity}

It was shown in Chapter 3 that the loss modulus is a father wavelet transform of the relaxation spectrum at unit scaling. In this section I will show that the storage modulus is also a special type of wavelet transform of the spectrum, and I shall introduce a whole family of wavelet transforms that occur naturally in the theory of linear viscoelasticity. Examples of dictionaries, consisting of father wavelets, will be introduced, that can be used to construct the continuous relaxation spectrum.

\subsubsection{Generalized wavelets}

Definition 5.3.1. (The generalized wavelet.) I define a generalized wavelet to be a function $\xi(x)$ such that $\phi^{0}(x)=\frac{d}{d x} \xi(x)$ is a father wavelet, with the property that

$$
0<\widehat{\phi}^{0}(0)=\left(C_{\psi}\right)^{\frac{1}{2}}<\infty
$$


The function

$$
\xi(x)=1+\tanh (x)
$$

is a generalized wavelet, since

$$
\phi^{0}(x)=\frac{d}{d x} \xi(x)=\operatorname{sech}^{2}(x)
$$

is a father wavelet, because

$$
\widehat{\phi^{0}}(0)=2
$$

from (6.2.6) and (6.2.26).

\subsubsection{Generalized wavelet transforms}

The generalized wavelet transform of $h \in L^{2}(\mathbb{R})$ at the time $t$ and scale $s$ is defined by the convolution product

$$
\begin{aligned}
{[G h](s, t) } & =\left(h \star \xi_{s}\right)(t), \\
\text { where } \quad \xi_{s}(x) & =\xi_{s, 0}(x)=\frac{1}{\sqrt{s}} \xi\left(\frac{x}{s}\right) .
\end{aligned}
$$

Equation (2.1.10) shows that in terms of log-frequency the storage modulus is a generalized wavelet transform of the continuous relaxation spectrum, at unit scaling $s=1$, i.e.

$$
\begin{aligned}
g_{1}(x) & =[G h](1, x)=(h \star \xi)(x) \\
\text { with } \quad \xi(x) & =\xi_{1}(x)=\xi_{1,0}(x)=1+\tanh (x)=1+\tanh (\ln \omega) .
\end{aligned}
$$

Furthermore, if

$$
\xi(x)=\arctan (\sinh (x))
$$


then

$$
\phi^{0}(x)=\frac{d}{d x} \xi(x)=\operatorname{sech}(x)
$$

Consequently, $\int_{-\infty}^{x} g_{2}\left(x^{\prime}\right) d x^{\prime}$ is a generalized wavelet transform of the continuous relaxation spectrum, at unit scaling $s=1$. These two generalized wavelets introduced above are plotted together in Fig. 6.9.

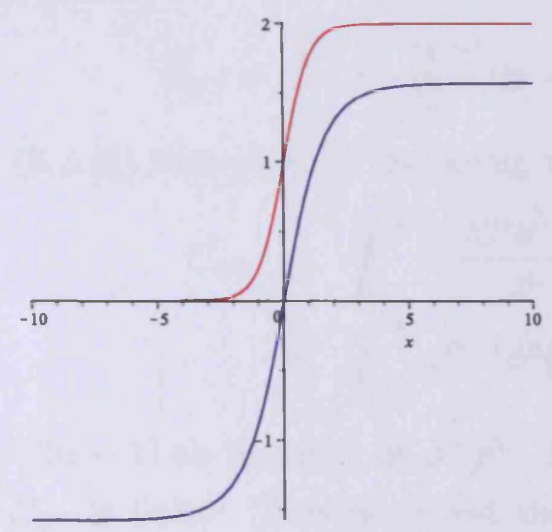

Figure 6.9: Two generalized wavelets. $\xi(x)=\tanh (x)$ (red) against $\xi(x)=$ $\arctan (\sinh (x))$ (blue).

\subsubsection{Differentiating wavelet transforms}

It has already been shown that, starting with a generalized wavelet $\xi(x)$, differentiation with respect to $x$ introduces a father wavelet $\phi^{0}(x)$. Then $\psi^{0}(x)$ is the mother wavelet associated with $\phi^{0}(x)$. I will now show that further levels of differentiation will always produce mother wavelets. Let

$$
\psi^{n}(x)=\frac{d^{n}}{d x^{n}} \phi^{0}(x) \quad(n \geq 1) .
$$


Then

$$
\begin{aligned}
\widehat{\psi^{n}}(0) & =\left[\frac{d^{n}}{d x^{n}} \phi^{0}(x)\right]^{\wedge}(0) \\
& =\left.\left[(i p)^{n} \widehat{\phi^{0}}(p)\right]\right|_{p=0} \\
& =0
\end{aligned}
$$

which shows that the function $\psi^{n}(x)$ has zero area. $\psi^{n}(x)$ is a mother wavelet if it is also true that

$$
C_{\psi^{n}}=\int_{0}^{\infty} \frac{\left.\widehat{\mid \psi^{n}}(p)\right|^{2}}{|p|} d p<\infty
$$

Substituting (6.3.11) into (6.3.13) and using table 1.1,

$$
\begin{aligned}
C_{\psi^{n}} & =\int_{0}^{\infty} \frac{\left|(i p)^{n} \widehat{\phi^{0}}(p)\right|^{2}}{|p|} d p \\
& =\int_{0}^{\infty} p^{2 n-1} \widehat{\phi^{0}}(p) d p
\end{aligned}
$$

which is the $(2 n-1)$-th moment of $\widehat{\phi}^{0}(p)$. As long as $\widehat{\phi}^{0}(p)$ decreases exponentially, $C_{\psi^{n}}$ is finite. This is indeed the case for $\phi^{0}(x)=\operatorname{sech}(x)$ or $\phi^{0}(x)=\operatorname{sech}^{2}(x)$.

Since

$$
\begin{aligned}
\frac{d^{n}}{d x^{n}}\left[h(x) \star \phi^{0}(x)\right] & =\frac{d^{n}}{d x^{n}} \int_{-\infty}^{\infty} h(t) \phi^{0}(x-t) d t \\
& =\int_{-\infty}^{\infty} h(t) \frac{d^{n}}{d x^{n}}\left(\phi^{0}(x-t)\right) d t \\
& =h(x) \star \frac{d^{n}}{d x^{n}} \phi^{0}(x)
\end{aligned}
$$

it follows that differentiating a father wavelet transform will produce a mother wavelet transform, and all further derivatives will always produce mother wavelet transforms.

If $\phi^{0}(x)$ is an even function of $x$ then, when $n$ is odd $\frac{d^{n}}{d x^{n}} \phi(x)$ will be an odd mother wavelet, and when $n$ is even $\frac{d^{n}}{d x^{n}} \phi(x)$ will be an even mother wavelet. 


\subsection{4 $n$-th level reconstruction formulae}

I have just shown that as we continue the process of differentiating wavelet transforms, I continue to get more and more wavelet transforms. I will now derive formulae for calculating reconstruction formulae for the continuous relaxation spectrum associated with the $n$-th level of differentiation. For the reconstruction formula at level $n$, I still require $\psi^{n}(x) \star \psi^{n}(x)$ and $\phi^{n}(x) \star$ $\phi^{n}(x)$. Using (3.1.3), I will now derive a useful formula for $\phi^{n}(x) \star \phi^{n}(x)$ :

$$
\begin{aligned}
\left|\left[\phi^{n}(x)\right]^{\wedge}(p)\right|^{2}=\left|\left[\phi^{n}(x) \star \phi^{n}(x)\right]^{\wedge}(p)\right| & =\int_{p}^{\infty} \frac{\left|\left[\psi^{n}(x)\right]^{\wedge}(\omega)\right|^{2}}{\omega} d \omega \\
& =\int_{p}^{\infty} \frac{\left|\left[\frac{d^{n}}{d x^{n}} \phi^{0}(x)\right]^{\wedge}(\omega)\right|^{2}}{\omega} d \omega \\
& =\int_{p}^{\infty} \frac{\left|(\omega)^{n}\left[\phi^{0}(x)\right]^{\wedge}(\omega)\right|^{2}}{\omega} d \omega \\
& =\int_{p}^{\infty} \omega^{2 n-1}\left|\left[\phi^{0}(x)\right]^{\wedge}(\omega)\right|^{2} d \omega \\
\therefore \frac{d}{d p}\left|\left[\phi^{n}(x) \star \phi^{n}(x)\right]^{\wedge}(p)\right| & =-p^{2 n-1}\left|\left[\phi^{0}(x) \star \phi^{0}(x)\right]^{\wedge}(p)\right| \\
\therefore\left|\left[x \phi^{n}(x) \star \phi^{n}(x)\right]^{\wedge}(p)\right| & =\left|\left[\frac{d^{2 n-1}}{d x^{2 n-1}}\left(\phi^{0}(x) \star \phi^{0}(x)\right)\right]^{\wedge}(p)\right| \\
\therefore \phi^{n}(x) \star \phi^{n}(x) & =\frac{1}{x} \frac{d^{2 n-1}}{d x^{2 n-1}}\left(\phi^{0}(x) \star \phi^{0}(x)\right) .
\end{aligned}
$$

Also, by generalizing equation (3.1.17), I obtain

$$
\psi^{n}(x) \star \psi^{n}(x)=\frac{d}{d x}\left[x\left(\phi^{n} \star \phi^{n}\right)(x)\right]
$$

and by substituting equation (6.3.16) into the above equation, I find that

$$
\begin{aligned}
\psi^{n}(x) \star \psi^{n}(x) & =\frac{d}{d x}\left[x\left(\frac{1}{x} \frac{d^{2 n-1}}{d x^{2 n-1}}\left(\phi^{0}(x) \star \phi^{0}(x)\right)\right)\right] \\
& =\frac{d^{2 n}}{d x^{2 n}}\left(\phi^{0}(x) \star \phi^{0}(x)\right) .
\end{aligned}
$$


At the $n$-th level of differentiation I can now express the Calderón-Mallat decomposition of the identity (3.2.10) using the cross-pair $\left\{\phi^{n}(x) \star \phi^{n}(x), \psi^{n}(x) \star\right.$ $\left.\psi^{n}(x)\right\}$ in terms of the autoconvolution of the original father wavelet $\phi^{0}(x)$

$$
\begin{aligned}
h(t)= & \frac{1}{C_{\psi^{n}}} \int_{0}^{\sigma}\left(h \star \psi_{s}^{n} \star \psi_{s}^{n}\right)(t) \frac{d s}{s^{2}}+\frac{1}{C_{\psi^{n}} \sigma}\left(h \star \phi_{\sigma}^{n} \star \phi_{\sigma}^{n}\right)(t) \\
= & \frac{1}{C_{\psi^{n}}} \int_{0}^{\sigma}\left[h(t) \star \frac{d^{2 n}}{d t^{2 n}}\left(\phi_{s}^{0}(t) \star \phi_{s}^{0}(t)\right)\right] \frac{d s}{s^{2}} \\
& +\frac{1}{C_{\psi^{n}} \sigma}\left[h(t) \star \frac{1}{t} \frac{d^{2 n-1}}{d t^{2 n-1}}\left(\phi_{\sigma}^{0}(t) \star \phi_{\sigma}^{0}(t)\right)\right],
\end{aligned}
$$

where $C_{\psi^{n}}$ is given by (6.3.14). Since equations (2.1.8) and (2.1.9) are infinitely often differentiable, there are infinitely many cross-pairs to choose from when attempting to recover the relaxation spectrum.

In particular, for

$$
\left(\phi^{0}(t) \star \phi^{0}(t)\right)=\operatorname{sech}(t),
$$

and $n=1$, the Mallat part of the above Calderòn-Mallat decomposition is proportional to

$$
h(t) \star \frac{1}{t} \frac{d}{d t} \operatorname{sech}(t)=h(t) \star t^{-1} \operatorname{sech}(t) \tanh (t) .
$$

Consequently, the continuous relaxation spectrum can be constructed from super-hyperbolic dictionaries consisting of atoms of the form $t^{-1} \operatorname{sech}(t) \tanh (t)$. The factor $t^{-1}$ means that the atoms have a slightly faster rate of decay than exponential - hence super-hyperbolic.

\subsection{Conclusion}

The main purpose of this chapter was to show that introducing a new dictionary of basis functions for recovering the continuous relaxation spectrum gave results that were consistent with those obtained with the sech dictionary, which we used originally. I have introduced the autoconvolution of 
$\operatorname{sech}(x)$, which is proportional to $x \operatorname{cosech}(x)$, as a sub-hyperbolic wavelet. I have shown in this chapter that it is another valid choice of father wavelet for constructing dictionaries, whose atoms can be used to represent the continuous relaxation spectrum. It satisfied the constraint of finite positive Riesz bounds, and its associated mother wavelet gave a tighter frame than the mother wavelet associated with $\operatorname{sech}(x)$, for larger sampling rates. For small sampling rates, however, the mother wavelet associated with $\operatorname{sech}(x)$ gave a tighter frame. The cross-pair of father and mother wavelet resulting from this new dictionary gave extremely small errors in the two-scale relations, even smaller than our original cross-pairing from Chapter 3.

We showed that, like the cross-pairing resulting from the sech dictionary, the new cross-pairing also required $0<\sigma<1$ to attain real-time integrability, and consequently meet the constraint of finite total viscosity. I have produced models for $g_{1}(x)$ and $g_{2}(x)$ for general values of $\sigma$, using the $x \operatorname{cosech}(x)$ dictionary, and by using the same set of real data which was used with the sech dictionary, I achieved very similar data fits and resulting spectra. The relaxation spectra obtained from the two different dictionaries using the optimal value of $\sigma$ was very similar, and gave consistency in my results. The main result to come out of this section of the chapter was that by using two different dictionaries, the search algorithm appears to choose a value of $\sigma$ which gives a similar shape of basis function for fitting both the storage and loss moduli data. Consequently, in this particular case, this results in a similar shape of basis function for the relaxation spectrum, as illustrated in Fig. 6.7(b).

Another important part of this chapter was to produce error terms in the delta sequence method of relaxation spectrum recovery. This was derived by using powers of sech as dictionaries for the spectrum. I was able to show that the error terms were in fact the Calderòn part of the Calderòn-Mallat decomposition of the identity, and I gave the integral form of these errors in 
terms of the physical variables $\omega$ and $\tau$.

The final part of this chapter introduced other wavelet transforms which arise naturally in the theory of linear viscoelasticity. It has been explained what happens during the process of differentiating wavelet transforms and I derived general formulae for linking the corresponding mother and father wavelet, at the $n$-th level of differentiation, with the original father wavelet in the unique father wavelet transform. An example has been given of superhyperbolic dictionaries, whose atoms can be used to represent the continuous relaxation spectrum. 


\section{Chapter 7}

\section{Other Algorithms}

In this chapter I will explore other algorithms for estimating the continuous relaxation spectrum from the dynamic data set PB1, using both sech and $x$ cosech dictionaries. The main purpose of this chapter is to discover whether different algorithms will give results that are consistent with those obtained from my search algorithm (introduced in Chapter 5), in the sense that they all point towards a bimodal relaxation spectrum for this particular polymer blend.

\subsection{Greedy Algorithms}

A greedy algorithm is an algorithm that is designed to search for an optimum solution along a heuristic approach. The choice made by a greedy algorithm may depend on choices made so far but not on future choices. It iteratively makes one greedy choice after another, reducing each given problem into a smaller one. A crucial property of a greedy algorithm is that it cannot choose the same basis function more than once. A greedy algorithm will enable me to construct the relaxation spectrum in a systematic way.

Many pursuit algorithms are available for sparse approximation and an 
excellent account of those available are given by Chen, Donoho and Saunders (2001 [21]). Examples include Basis Pursuit (BP), Best Orthogonal Basis (BOB) and Orthogonal Matching Pursuit (OMP). The greedy algorithm which we have chosen is OMP.

\subsubsection{Choosing $\Lambda$ by Orthogonal Matching Pursuit (OMP)}

OMP is an iterative process for selecting a subset of atoms from a dictionary. It has been in use for about four decades, where it was used originally in Statistics under the name of Forward Selection Algorithm [57]. A recent account is given in the work by Tropp and Gilbert in signal analysis [73].

The structure of this problem is already set up for OMP. We use OMP to choose iteratively which vectors of the sub-dictionay $\chi$ (introduced in Section 5.4) give the best simultaneous least squares fit to the data for the storage and loss moduli. The algorithm works as follows: [73]

INPUT:

- An $N$ x $d$ measurement matrix $\chi$

- An $N$-dimensional data vector $\mathbf{g}$

- The sparsity level $m$ of the ideal signal

\section{OUTPUT:}

- An estimate $\hat{\mathbf{s}} \in \mathbb{R}^{N}$ for the ideal signal

- A set $\Lambda_{m}$ containing $m$ elements from $\{1, d\}$

- An $N$-dimensional approximation $\mathbf{a}_{\mathbf{m}}$ of the data vector $\mathbf{g}$

- An $N$-dimensional residual $\mathbf{r}_{m}=\mathbf{g}-\mathbf{a}_{m}$ 
PROCEDURE:

- Step 1: Initialize the residual $\mathbf{r}_{0}=\mathbf{g}$, the index set $\Lambda_{0}=\emptyset$, and the iteration counter $t=1$.

- Step 2: Find the index $\lambda_{t}$ that solves the easy optimization problem

$$
\lambda_{t}=\arg \max _{\{k=1, \ldots, d\}}\left|\left\langle\mathbf{r}_{t-1}, \chi_{k}\right\rangle\right|
$$

- Step 3: Augment the index set $\Lambda_{t}=\Lambda_{t-1} \cup\left\{\lambda_{t}\right\}$ and the matrix of chosen atoms $\chi_{t}=\left[\begin{array}{ll}\chi_{t-1} & \chi_{\lambda_{t}}\end{array}\right]$. The convention used is that $\chi_{0}$ is an empty matrix.

- Step 4: Solve a least squares problem to obtain a new signal estimate:

$$
\mathbf{x}_{t}=\arg \min _{\mathbf{x}}\left\|\chi_{t} \mathbf{x}-\mathbf{g}\right\|_{2}
$$

- Step 5: Calculate the new approximation of the data and the new residual:

$$
\begin{aligned}
& \mathbf{a}_{t}=\chi_{t} \mathbf{x}_{t} \\
& \mathbf{r}_{t}=\mathbf{g}-\mathbf{a}_{t} .
\end{aligned}
$$

- Step 6: Increment $t$, and return to Step 2 if $t<m$.

- Step 7: The estimate $\hat{\mathbf{s}}$ for the ideal signal has nonzero indices at the components listed in $\Lambda_{m}$. The value of the estimate $\hat{\mathbf{s}}$ in component $\lambda_{j}$ equals the $j$-th component of $\mathbf{x}_{t}$ 


\subsubsection{PB1 results}

\section{Case 1: sech dictionary}

When using the model for $h$ from equations (5.2.4)-(5.2.6):

$$
\begin{aligned}
h(t) & =\sum_{k \in \Lambda_{m}} b_{k} \Gamma\left(\frac{t-x_{k}}{\sigma}\right), \\
\text { where } \Gamma(t) & =\operatorname{sech}(t) \\
\text { and } x_{k} & =x_{0}+k a
\end{aligned}
$$

we found that with PB1 data, the most stable results occurred when $\sigma=\frac{1}{2}$ and $a=0.1$. Five iterations of OMP are required in order to bring the root mean square error (RMS) below two per cent. The fit for $G^{\prime}(\omega)$ and $G^{\prime \prime}(\omega)$, when $m=5$, is displayed in Fig. 7.1(a). The resulting continuous relaxation spectrum is then plotted in Fig. 7.1(b). There is a slight wobble in the largest of the peaks in Fig. 7.1(b) and a small negative lobe at very small relaxation times. The value of $m$ required to get a respectable fit to the data using OMP is one higher than in my own search algorithm, introduced in Chapter 5. Therefore, I have a less sparse approximation using OMP.

As I increase $m$ from 5 to 8 the RMS decreases from $1.79 \%$ to $1.06 \%$ (see Table 7.1). The resulting spectra are relatively stable, as shown in Fig. 7.1(c). However, when $m$ becomes too large we get noise amplification. This is evident in Fig. 7.1(d), where $m$ is increased to nine. As a result I get an extra peak in the spectrum and more small negative lobes. This is because $m$, as well as $\sigma$, is acting as a regularization parameter in this greedy algorithm. If $m$ is too small I get a very poor fit to the data, whereas if $m$ is too large I start to fit the noise in the data and consequently get unrealistic approximations for the relaxation spectrum. $\sigma$ acts in a very similar manner as illustrated in earlier chapters where I have used my own search algorithm. 



(a) Data fit for $G^{\prime}(\omega)$ and $G^{\prime \prime}(\omega)$. (b) Relaxation Spectrum. $m=5$. $m=5$ and RMS error $=1.79 \%$.


(c) Relaxation Spectrum. $m=6-$

(d) Relaxation Spectrum. $m=9$.

8. $m=6$ (green), $m=7$ (red), $m=8$ (blue).

Figure 7.1: OMP with sech dictionary; $\sigma=\frac{1}{2}, a=0.1$. 


\begin{tabular}{|c|c|c|c|c|c|}
\hline $\mathrm{m}$ & 5 & 6 & 7 & 8 & 9 \\
\hline $\mathrm{RMS}$ & 1.79 & 1.60 & 1.18 & 1.06 & 0.93 \\
\hline
\end{tabular}

Table 7.1: Values of RMS for progressing iterations of OMP, where $\sigma=1 / 2$ and $a=0.1$.

\section{Case 2: $x$ cosech dictionary}

When using the model for $h$ of the form

$$
\begin{aligned}
h(t) & =\sum_{k \in \Lambda_{m}} c_{k} \Gamma\left(\frac{t-x_{k}}{\sigma}\right), \\
\text { where } \Gamma(t) & =2 t \operatorname{cosech}(t),
\end{aligned}
$$

I discovered that the relaxation spectra that I obtained was much more stable than in case 1 . I obtained the most stable results with $\sigma=1 / 4$ and $a=0.2$. When compared to case 1 , OMP required one extra iteration to bring the RMS error below $2 \%$, but once it did so, the spectra from iterations 6-8 were extremely stable, as shown in Fig. 7.2(c). For these progressing values of $m$, the RMS error changes much less than in case 1 (see Table 7.2).

When $m=6$, the data fit for $G^{\prime}(\omega)$ and $G^{\prime \prime}(\omega)$ is given in Fig. 7.2(a) and the resulting spectrum is plotted in Fig. 7.2(b). As in case 1, we again see how $m$ acts as a regularization parameter. Making $m$ too large leads to noise amplification. This is displayed in Fig. 7.2(d), where $m=9$.

The optimal value of $\sigma$ obtained from OMP for the $x$ cosech dictionary is the same as obtained via the search algorithm in Chapter 6. OMP again gives a less sparse approximation than my search algorithm, since the smallest value of $m$ which gives a respectable fit to the data with this dictionary is $m=6$. 

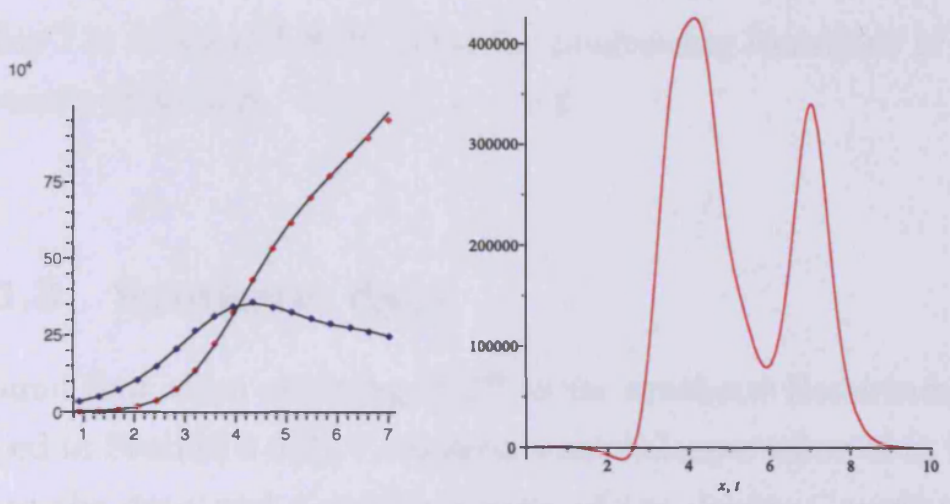

(a) Data fit for $G^{\prime}(\omega)$ and $G^{\prime \prime}(\omega)$. (b) Relaxation Spectrum. $m=6$. $m=6, \mathrm{RMS}=1.19 \%$.

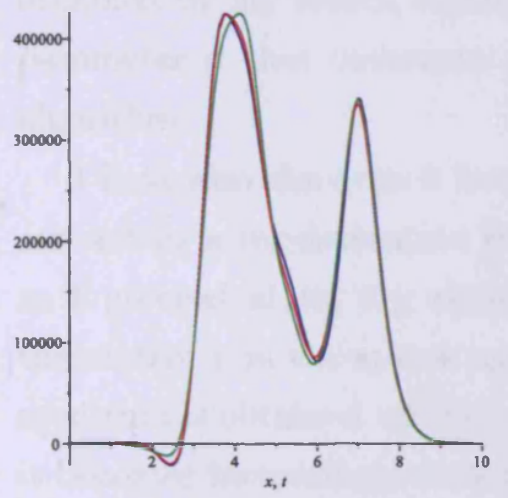

(c) Relaxation Spectrum. $m=6-$

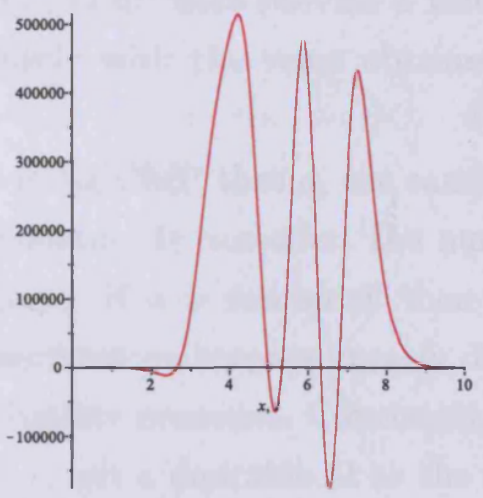

(d) Relaxation Spectrum. $m=9$. 8.

Figure 7.2: OMP with $x$ cosech dictionary. $\sigma=1 / 4, a=0.2$. 


\begin{tabular}{|c|c|c|c|c|}
\hline $\mathrm{m}$ & 6 & 7 & 8 & 9 \\
\hline $\mathrm{RMS}$ & 1.19 & 1.13 & 1.12 & 1.01 \\
\hline
\end{tabular}

Table 7.2: Values of RMS error for progressing iterations of OMP using the $x$ cosech dictionary. $\sigma=1 / 4, a=0.2$.

\subsubsection{Synthetic data}

I found that when applying OMP to the synthetic Honerkamp A data (introduced in Section 5.6.2), I required a much larger value of $m$ to get a suitable fit to the data and a good recovery of the double-Gaussian spectrum. Although OMP gives an approximation which is much less sparse than that obtained by my search algorithm, OMP does provide a value of the scaling parameter $\sigma$ that compares closely with the value obtained by my search algorithm.

I have also discovered from using OMP that $a$, the sampling parameter, can act as a regularization parameter. It can affect the numerical stability as I proceed along the algorithm. If $a$ is too small then the columns of the matrix $\chi$ in the sparse approximation become linearly dependent, and a spectrum is obtained which is absolute nonsense. Conversely, as a gets larger it becomes increasingly difficult to get a desirable fit to the data.

\subsection{Parity reduction}

Using OMP, the shape of the relaxation spectrum for PB1 is consistent with results obtained from my search algorithm. One of the disadvantages of using OMP is that, when compared to my search algorithm, it requires more basis elements to fit the dynamic data to an acceptable level. I now introduce 
a method to try to improve upon this fault of OMP. The idea behind parity reduction is to exploit symmetry of atoms, and hence work with symmetric and anti-symmetric parts of the data. Thus, I split the storage and loss moduli into odd and even parts and fit the models to these parts separately. For the PB1 data there are $N=17$ data points $\xi_{i}, i=\{1, \ldots, N\}$. The odd and even parts of $G^{\prime}(\omega)$ are given respectively by

$$
\begin{aligned}
G_{O}^{\prime}\left(\xi_{i}\right) & =\frac{G^{\prime}\left(\xi_{i}\right)-G^{\prime}\left(\xi_{N-i}\right)}{2} \\
G_{E}^{\prime}\left(\xi_{i}\right) & =\frac{G^{\prime}\left(\xi_{i}\right)+G^{\prime}\left(\xi_{N-i}\right)}{2} .
\end{aligned}
$$

Similarly, the odd and even parts of $G^{\prime \prime}(\omega)$ are given respectively by

$$
\begin{aligned}
& G_{O}^{\prime \prime}\left(\xi_{i}\right)=\frac{G^{\prime \prime}\left(\xi_{i}\right)-G^{\prime \prime}\left(\xi_{N-i}\right)}{2} \\
& G_{E}^{\prime \prime}\left(\xi_{i}\right)=\frac{G^{\prime \prime}\left(\xi_{i}\right)+G^{\prime \prime}\left(\xi_{N-i}\right)}{2} .
\end{aligned}
$$

After parity reduction, I use OMP to choose the basis functions to fit the data, separately, and consequently reconstruct $h$.

I discovered that, using either dictionary, I obtain much better results by fitting the models to the odd and even parts of $G^{\prime \prime}(\omega)$ first, and subsequently constructing $G^{\prime}(\omega)$ from these results, rather than the other way around.

\section{Case 1: sech dictionary}

By fitting the models to $G_{E}^{\prime \prime}(\omega)$ with $\sigma=\frac{1}{2}$ again, I obtained an excellent fit to the data of $0.37 \%$ with just three basis functions. This result is plotted in Fig. 7.3(a), along with the independent fit to $G_{O}^{\prime \prime}(\omega)$, where I got an RMS error of $4.67 \%$ with four basis functions. Two of the basis functions were the same in each of the independent approximations, so that when I combine the two to form an approximation for $G^{\prime \prime}(\omega)$, I only have five separate basis functions. The overall fit to the $G^{\prime \prime}(\omega)$ data is $0.60 \%$ and is plotted in 
Fig. 7.3(b) along with the subsequent fit to the $G^{\prime}(\omega)$ data, which has an extremely large RMS error of $11.8 \%$. Although the fit looks extremely good the problem is that there is a large percentage difference in the $G^{\prime}(\omega)$ data at low frequencies. The overall fit to the data is $8.35 \%$, which is much poorer than results obtained by using OMP on its own. The resulting relaxation spectrum is also shown in Fig. 7.3(b).

A much better fit to the data is obtained, however, by using the five basis functions found from the above method, but then allowing a simultaneous fit to the storage and loss moduli. This gives a superb fit to the overall data of $1.45 \%$, and is plotted in Fig. $7.3(\mathrm{c})$, along with the resulting spectrum. The separate relaxation spectra, obtained from the two different methods, are plotted together in Fig. 7.3(d). The similarity implies that our 'tweaked' method of finding the coefficients in the approximation has very little difference in the appearance of $h$, but it dramatically increases the quality of the data fit. The small negative lobe is reduced in size, too, which is also desirable.

\section{Case 2: $x$ cosech dictionary}

I obtained similar results using the $x$ cosech dictionary. By fitting the models to $G_{E}^{\prime \prime}(\omega)$ with $\sigma=\frac{1}{4}$, I obtained a superb fit to the data of $0.48 \%$ with five basis functions. This result is plotted in Fig. 7.4(a), along with the independent fit to $G_{O}^{\prime \prime}(\omega)$, where I got an RMS error of $4.70 \%$ with four basis functions. Two of the basis functions were the same in each of the independent approximations, so that when I combine the two to form an approximation for $G^{\prime \prime}(\omega)$, I only have five separate basis functions. The overall fit to the $G^{\prime \prime}(\omega)$ data is $0.67 \%$ and is plotted in Fig. 7.4(b) along with the subsequent fit to the $G^{\prime}(\omega)$ data, which has an RMS error of $5.60 \%$. The overall fit to the data is $3.99 \%$, which is much poorer than results obtained by using OMP on its own. The resulting relaxation spectrum is also shown 

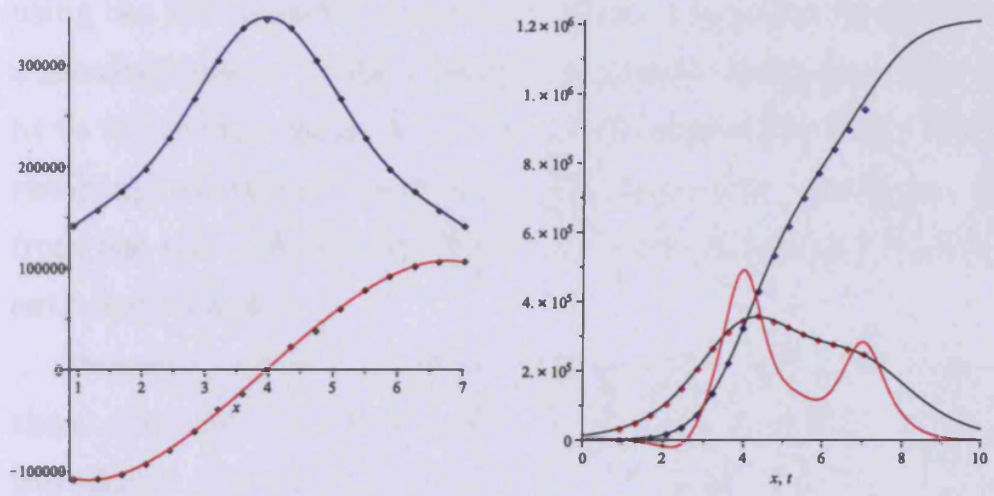

(a) Independent fits to $G_{E}^{\prime \prime}$ (blue, (b) Fit to $G^{\prime \prime}(m=5)$, subsequent $m=3)$ and $G_{O}^{\prime \prime}($ red, $m=4)$. fit to $G^{\prime}(m=5)$ and resulting spectrum.


(c) Simultaneous fit to $G^{\prime}$ and $G^{\prime \prime}$ (d) Relaxation spectra comparison. $(m=5)$ and subsequent spectrum. Spectrum from simultaneous fit in blue.

Figure 7.3: OMP and parity reduction, using sech dictionary. 
in Fig. 7.4(b).

As with the sech dictionary, a much better fit to the data is obtained by using the five basis functions found from the above method, but then allowing a simultaneous fit to the storage and loss moduli data. This gives an excellent fit to the overall data of $1.11 \%$, and is plotted in Fig. 7.4(c), along with the resulting relaxation spectrum. The separate relaxation spectra, obtained from the two different methods, are plotted together in Fig. 7.4(d), and are extremely similar.

However, when I compare these results with the $x$ cosech dictionary to those obtained by using OMP on its own (see Fig. 7.2), parity reduction introduces a small third peak into the approximation for $h$, which was not there before.

\subsection{Density estimation}

\subsubsection{Introduction}

In probability and statistics, density estimation is the construction of an estimate, based on observed data, of an unobservable underlying probability density function. A variety of approaches to density estimation are used, including Parzen windows [63] and a range of data clustering techniques, including vector quantization [37]. The most basic form of density estimation is a rescaled histogram. More details on density estimation can be found in Bowman and Azzalini [18], and Silverman [69]

From (2.1.9), it ẘ $g_{2}(x)=\int_{-\infty}^{\infty} \operatorname{sech}(x-t) h(t) d t$. ${ }^{\text {uld be expressed as }}$

$$
g_{2}(x)=\int_{-\infty}^{-\infty} \operatorname{sech}(x-t) h(t) d t .
$$

This expression shows that the loss modulus is a smoothed estimate of the continuous relaxation spectrum, where $\operatorname{sech}(x-t)$ is the kernel and $h(t)$ is the unknown density function. 

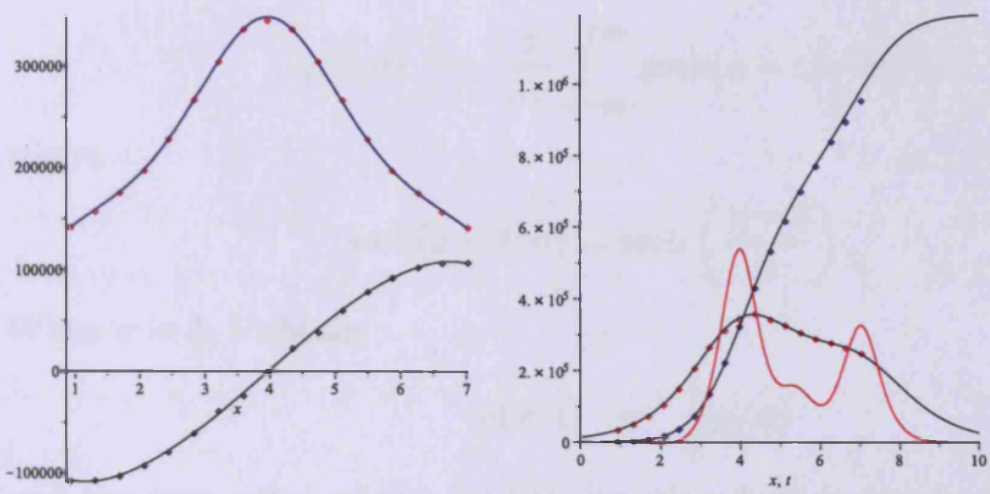

(a) Independent fits to $G_{E}^{\prime \prime}$ (blue, (b) Fit to $G^{\prime \prime}(m=5)$, subsequent $m=5)$ and $G_{O}^{\prime \prime}$ (red, $\left.m=4\right) . \quad$ fit to $G^{\prime}(m=5)$ and resulting spectrum.
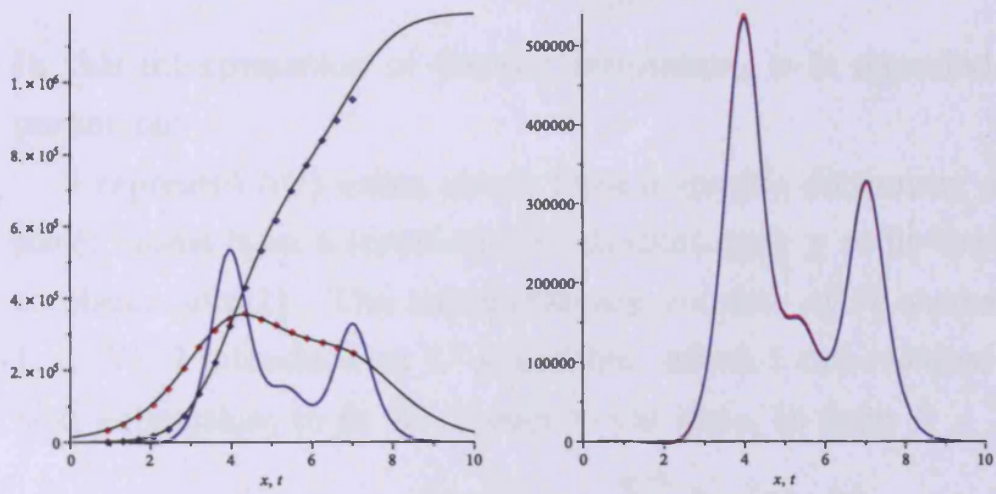

(c) Simultaneous fit to $G^{\prime}$ and $G^{\prime \prime}$ (d) Relaxation spectra comparison. $(m=5)$ and subsequent spectrum . Spectrum from simultaneous fit in blue.

Figure 7.4: OMP and parity reduction, using $x \operatorname{cosech}$ dictionary. 


\subsubsection{Kernel density approach}

I define $g(x ; \sigma)$ by

$$
g(x ; \sigma)=\frac{1}{\sigma \pi} \int_{-\infty}^{\infty} \operatorname{sech}(x-t ; \sigma) h(t) d t
$$

where

$$
\operatorname{sech}(x-t ; \sigma)=\operatorname{sech}\left(\frac{x-t}{\sigma}\right) .
$$

When $\sigma=1$, I obtain

$$
g(x ; 1)=\frac{1}{\pi} g_{2}(x)
$$

and the true value of the density function $h(x)$ is obtained in the limit as $\sigma \rightarrow 0$ :

$$
h(x)=\lim _{\sigma \rightarrow 0} g(x ; \sigma) .
$$

In this interpretation of density estimation, $\sigma$ is regarded as a smoothing parameter.

I represent $h(t)$ using atoms from a specific dictionary and subsequently select atoms from a transformed sub-dictionary $\chi$ to fit the loss moduli data to obtain $g(x ; 1)$. The sub-dictionary consists of $N$ atoms $\chi\left(x-\xi_{i}\right),(i=$ $1, \ldots, N)$. I introduce an $L^{1}$-algorithm, which I call residual bisection, in the next subsection, to fit the model to the data, to form

$$
g(x ; 1)=\sum_{k \in \Lambda_{m}} b_{k} \chi\left(x-\xi_{i}\right) .
$$

I shall use PB1 data and a specific model for $h(t)$, so that I can compare density estimation results with those obtained from my search algorithm in Chapter 5, and from OMP earlier in this chapter. I then re-parameterize to form $g(x ; \sigma)$. I decrease $\sigma$ towards zero and observe what happens. Since the problem is ill-posed, the best estimate for $h$ will not be obtained when $\sigma=0$, but at some value $0<\sigma<1$. 


\subsubsection{Residual bisection}

The main objective of this algorithm is to avoid negative lobes in the distribution of $h$. Residual bisection is a matching pursuit algorithm based on $L^{1}$, unlike OMP, which is based on an $L^{2}$-algorithm. However, like OMP, residual bisection is also an iterative process for selecting a subset of atoms from a transformed dictionary. Residual bisection can choose the same atom more than once, which is a property that greedy algorithms like OMP do not have. These atoms are centred at the data points $\xi_{i}$, where $i=1, . ., N$.

Residual bisection uses as many atoms from a sub-dictionary as it can to fit the data without giving negative coefficients in the representation. Because this pursuit algorithm selects as many basis functions as it possibly can, it acts in the opposite manner of the search algorithm that I introduced in Chapter 5, which has the underlying principle of sparsity.

The algorithm works as follows:

\section{PROCEDURE:}

- Step 1: Choose $k_{1} \in\{1, \ldots, N\}$ such that $g_{2}\left(\xi_{k_{1}}\right) \geq g_{2}\left(\xi_{k}\right) \quad \forall k \in$ $\{1, \ldots, N\}$. Set $k_{1} \in \Lambda_{m}$. Form the first approximation of $g(x ; 1)$, namely

$$
g(x ; 1)_{1}=b_{k_{1}} \chi\left(x-\xi_{k_{1}}\right)
$$

where

$$
b_{k_{1}}=\frac{1}{2} g_{2}\left(\xi_{k_{1}}\right)
$$

Set

$$
\operatorname{res}\left(\xi_{k}\right)_{1}=g_{2}\left(\xi_{k}\right)-g(x ; 1)_{1}
$$


- Step 2: Choose $k_{2} \in\{1, \ldots, N\}$ such that $\operatorname{res}\left(\xi_{k_{2}}\right)_{1} \geq \operatorname{res}\left(\xi_{k}\right)_{1} \quad \forall k \in$ $\{1, \ldots, N\}$. Set $k_{2} \in \Lambda_{m}$. Form the second approximation of $g(x ; 1)_{2}$, namely

$$
g(x ; 1)_{2}=b_{k_{1}} \chi\left(x-\xi_{k_{1}}\right)+b_{k_{2}} \chi\left(x-\xi_{k_{2}}\right)
$$

where

$$
b_{k_{2}}=\frac{1}{2} \operatorname{res}\left(\xi_{k_{2}}\right)_{1}
$$

Set

$$
\operatorname{res}\left(\xi_{k}\right)_{2}=g_{2}\left(\xi_{k}\right)-g(x ; 1)_{2}
$$

- Step 3 ... Step $\mathbf{r}+1$ : Repeat step 2 for incremented $i(i=3, \ldots, r+1)$. Stop at step $r+1$ when $\exists l \in\{1, \ldots, N\}$ such that $\operatorname{res}\left(\xi_{l}\right)_{r+1} \leq 0$. Choose

$$
\begin{aligned}
g(x ; 1)=g(x ; 1)_{r} & =\sum_{i=1}^{r} b_{k_{i}} \chi\left(x-\xi_{k_{i}}\right) \\
& =\sum_{k \in \Lambda_{m}} b_{k} \chi\left(x-\xi_{k}\right),
\end{aligned}
$$

where there are $m \leq r$ atoms selected from the transformed dictionary.

\subsubsection{Density estimation results for PB1 data}

I use a Dirac-delta model for $h(t)$, namely

$$
h(t)=\sum_{k} a_{k} \delta(t-x[k]) .
$$

Using (7.3.2), I obtain

$$
g(x ; \sigma)=\frac{1}{\sigma \pi} \sum_{k} \operatorname{sech}\left(\frac{x-x[k]}{\sigma}\right) .
$$


Since the problem is ill-posed, the best estimate for $h$ will not be obtained when $\sigma=0$, but at some value $0<\sigma<1$. Thus the kernel density approach gives a smoothed estimate for the density function $h(t)$, expressed as a sum of dilated and translated sech functions. I fitted the model $g(x ; 1)$ to the loss moduli data using residual bisection, which used six basis functions and gave a fit to the data with an RMS error of $2.59 \%$. The fit to the data $(\pi g(x ; 1))$ is plotted in blue in Fig. $7.5(\mathrm{a})$ along with $g(x ; 1)$ (the smoothed estimate for the relaxation spectrum when $\sigma=1$ ). Although the fit is poor when compared to other algorithms I have used, the subsequent information it gives us about the spectrum as I decrease $\sigma$ in the re-parameterize model for $g(x ; \sigma)$ agrees with my previous results. As $\sigma$ is decreased, the density function change from being almost unimodal (Fig. 7.5(a)) when $\sigma=1$, to a bimodal density (Fig. 7.5(b)), until $\sigma$ is between the values of 0.4 and 0.5 (Fig. 7.5(c)), where the density function starts to display a third peak. This agrees with my results from PB1 data using the search algorithm in Chapter 5. In that chapter I explained why I thought that the relaxation spectrum for this particular viscoelastic material was bimodal, and I used homotopic approximation to obtained a value of $\sigma=0.45$ as the smallest value of $\sigma$ for which the spectrum was still bimodal. Using density estimation, I find that by estimating the continuous relaxation spectrum as a sum of dilated and translated sech functions, I obtain the same smallest value of $\sigma$ for which the spectrum is still visually bimodal (see Fig. 7.5(d)). As I decrease $\sigma$ further towards the value zero (Fig. 7.6(a)) I obtain more modes in the approximation for $h(t)$ and when $\sigma=0 \mathrm{I}$ obtain the six delta functions displayed in Fig. 7.6(b). 

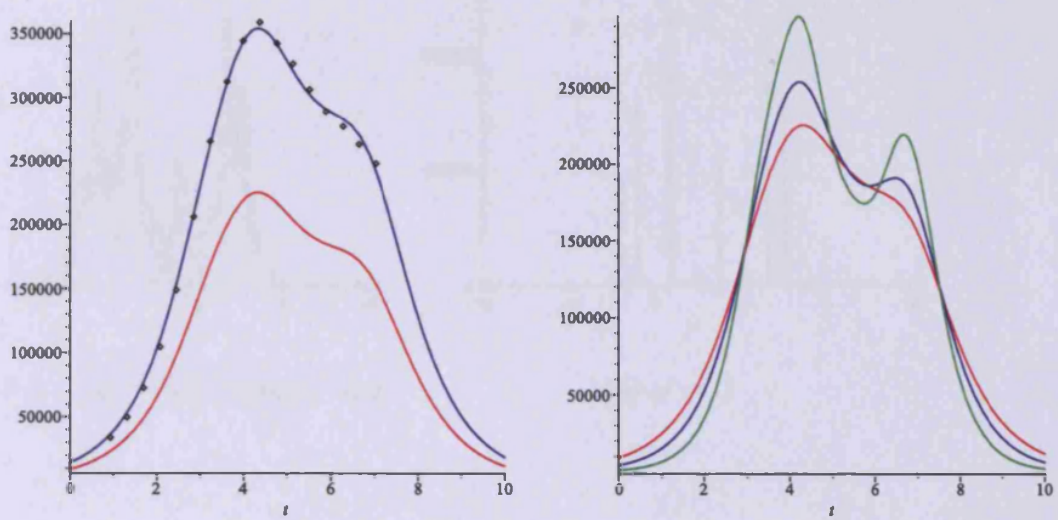

(a) $\sigma=1$

(b) $\sigma=1$ (red), 0.8 (blue), 0.6 (green).
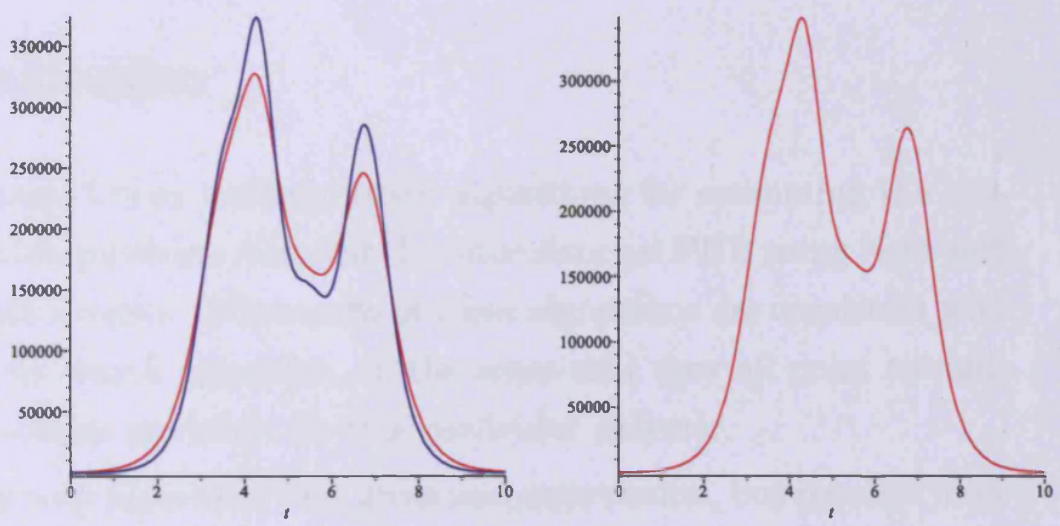

(c) $\sigma=0.5$ (red), 0.4 (blue).

(d) $\sigma=0.45$

Figure 7.5: Density Estimation. 




(a) $\sigma=0.3$ (red), 0.2 (blue), 0.1 (green).

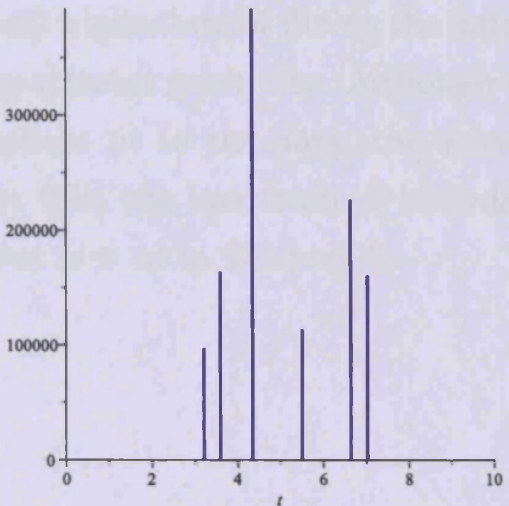

(b) $\sigma=0$

Figure 7.6: Density Estimation.

\subsection{Conclusion}

In this chapter, I have explored other algorithms for estimating the continuous relaxation spectrum from the dynamic data set $\mathrm{PB} 1$, using both sech and $x$ cosech dictionaries. The results of these algorithms are consistent with the results of my search algorithm, in the sense that they all point towards a bimodal relaxation spectrum for this particular polymer.

OMP is a greedy algorithm that gives adequate results, but requires more basis elements than our search algorithm to fit the data to an acceptable level. A disadvantage of this algorithm, when using PB1 data, is that it predicted a non-physical negative lobe in the spectrum. Noise amplification also became an issue if $m$ was too big.

I introduced a kernel density approach for recovering the continuous relaxation spectrum from the loss moduli data, and I showed that the loss modulus is a smoothed estimate for the relaxation spectrum. I introduced 
residual bisection, a simple pursuit algorithm for fitting the data and ensuring that the subsequent spectrum maintains positivity. Although this algorithm made it difficult to get an excellent fit to the data, the subsequent results added weight to my conclusion that the spectrum is bimodal, and it also predicted the same optimal value of $\sigma$ as in Chapter 5 . 


\section{Chapter 8}

\section{Conclusions}

In this thesis I have developed a new method for continuous relaxation spectrum recovery through wavelet analysis. One of the main results of this thesis is showing that wavelet analysis establishes natural models for the continuous relaxation spectrum. It has been shown that there exist wavelet transforms which are intrinsic to the theory of linear viscoelasticity, which give rise to these natural models. In particular, I have shown that the loss modulus is a father wavelet transform of the continuous relaxation spectrum at unit scaling.

I have explained how Calderón-Mallat decomposition allows me to express the continuous relaxation spectrum as a sum of mother and father wavelets. I have introduced a modification of Calderón-Mallat decomposition, defining a new way of pairing mother and father wavelets from different families, which I call cross-pairing. In particular, I have shown that the mother and father wavelet cross-pairing $\left(\psi^{\star}(x), \phi^{\star}(x)\right)=(\operatorname{sech}(x)[1-x \tanh (x)], \operatorname{sech}(x))$ satisfies the respective rigorous constraints of wavelet frames and Riesz bases. In particular, it has been shown that the mother wavelets associated with $\operatorname{sech}(x)$, in both the conventional Calderón-Mallat context and the crosspairing context, have tighter frames than the well-known Mexican hat wavelet 
for frame sampling rates $a \leq 1$. Furthermore, it has been shown that these cross-paired wavelets can be used to construct an essential multiresolution analysis. The concept of real-time integrability of wavelets (RTI wavelets) was introduced, to ensure that the finite viscosity constraint of a viscoelastic fluid is maintained. It was shown that the basis elements of the spectrum introduced in Chapter 3 are only RTI for $0<\sigma<1$. I introduced sech triplets as a subspace of the Riesz basis $V_{0}$, to fulfill the finite viscosity constraint when using $1<\sigma<3$.

I have produced models for the storage and loss moduli to fit the dynamic data. As long as $\sigma$ is rational, all of the required convolutions can be calculated analytically via residue calculus. I introduced a Gaussian spectrum and calculated noise-free dynamic data, as a test case for the models. It was shown that the Gaussian data could be fitted with very good accuracy using the scaling function (or father wavelet) part of the spectrum alone. Calderón enrichment was introduced to show that exceptional accuracy could be attained.

I have shown that recovery of the continuous relaxation spectrum is an exponentially ill-posed inverse problem, and consequently explained the need for regularization in the recovery process. I have introduced the regularization mechanisms acting in this particular method of relaxation spectrum recovery. The most important regularization parameters involved are the scale $\sigma$ and the sparsity $m$. Using both real and synthetic data, it is evident that if the value of $\sigma$ is too high, a poor fit to the data is achieved, and not enough information about the resulting spectrum is captured. Conversely, if $\sigma$ is too small, substantial superresolution is encountered, a concept that has been explored in this thesis. It has also been explained how the sparsity $m$ and the sampling parameter $a$ act as regularization parameters.

To select an optimal value of $\sigma$, for a chosen value of $m$, I select the smoothest spectrum that fits the data to an acceptable tolerance. If I choose 
$\sigma$ small enough I can always obtain $m$ peaks in the distribution, but not all of these peaks are supported by the underlying data. My philosophy is to choose the smallest number of peaks that give an acceptable fit to the data.

When it is clear that the optimal level of resolution is $\sigma \neq \frac{1}{n}(\sigma<1)$, I have developed a method for choosing a more accurate optimal value of $\sigma$. I construct a homotopic approximation for the spectra and data fits, linearly interpolating between the originally selected value of $\sigma$ (where the spectrum contains the smallest number of peaks that gives an acceptable fit to the data) and the nearest known smaller value of $\sigma$ (where we have at least one extra peak). I then select the optimal value of $\sigma$ by choosing the spectrum in this range which has the smallest total curvature.

It has been shown that super-resolution is an important factor when selecting an optimal value of $\sigma$. Super-resolution occurs when the Fourier transform of the recovered function $h(t)$ lies outside the Nyquist range of the data. When recovering the continuous relaxation spectrum from experimental data, I have tried to avoid super-resolution as much as possible. I believe that a value of super-resolution less than $1 \%$ is an acceptable value, since zero super-resolution is impossible when estimating the spectrum as a sum of hyperbolic wavelets.

The concepts of sparse approximation and dictionaries have been introduced and implemented in this thesis. One of the main results of the entire thesis is that, with an appropriate choice of the scaling parameter $\sigma$, the spectrum can be recovered with an acceptable level of accuracy using only the scaling function part of the spectrum. Consequently, Calderón enrichment is not required. This is particularly evident when dealing with noisy dynamic data when the resolution of the mother wavelets required is too high for the level of noise in the data, and leads to non-physical negative lobes in the spectrum.

In Chapter 6, I have shown that there are a whole family of wavelet 
transforms occurring naturally in the theory of linear viscoelasticity. Subsequently, I introduced other dictionaries that consisted of valid choices of father wavelet to construct the spectrum with. In particular, I introduced the auto-convolution of $\operatorname{sech}(x)$ as a viable option. I showed that with an appropriate value of $\sigma$, I could produce results that were consistent with those obtained with the sech dictionary. I conclude that for any valid, sensible choice of hyperbolic father wavelet, one should be able to produce similar results to the sech dictionary, provided an appropriate value of $\sigma$ is used.

In Chapter 7, I have explored other algorithms for fitting the dynamic data set for PB1. The results of these algorithms are consistent with the results of my search algorithm, in the sense that they all point towards a bimodal relaxation spectrum for this particular polymer blend.

Also in this thesis, I have introduced generalized formulae for double integral inversion formulae relating moments of the relaxation spectrum to data involving $\omega^{m} G^{*}(\omega)$. I have given a specific example of a well-behaved relaxation spectrum that does not have a Maclaurin series. Furthermore, I have shown in this thesis that Calderón-Mallat decomposition can be used to derive the error terms in the Delta Sequence method of relaxation spectrum recovery.

I have developed a fresh approach to numerical deconvolution in this thesis, which is applicable to the deconvolution of noisy data where there is a strong level of ill-posedness in the associated inverse problem. In my $\mathrm{PhD}$ I have confined attention to an exponentially ill-posed inverse problem in linear viscoelasticity, which also has an analogue in the relaxation of dielectric materials. However, the methodology I have developed can be used more generally, and I believe there is a possibility of potential impact of my work in other application areas of signal and image processing. 


\section{Bibliography}

1. Abbiss J (1977). Photon correlation spectroscopy and velocimetry. (Edited by H.Z. Cummins, E.R. Pike) New York, 386-423.

2. Addison PS (2002). The illustrated wavelet transform handbook. IOP.

3. Aldroubi A, Unser M (1992). Families of wavelet transforms in connection with Shannon's sampling theory and the Gabor transform. Wavelets: A tutorial in Theory and Applications (C.K.Chui, Ed.), Academic Press, Boston, pp.509-528.

4. Ancey C, Jorrot $\mathrm{H}$ (2001). Yield stress for particle suspensions within a clay dispersion. J Rheol 45:297-319.

5. Anderssen RS, Davies AR (2001). Simple moving-average formulae for the direct recovery of the relaxation spectrum. J Rheol 45:1-27.

6. Anderssen RS, Saiful A Husain, Loy RJ (2004). The Kohlrausch function: properties and applications. ANZIAM 45:800-816.

7. Anderssen RS, Saiful A Husain (2005). Modelling the relaxation modulus of linear viscoelasticity using Kohlrausch functions. J Non-Newton Fluid Mech 125:159-170.

8. Anderssen RS, Saiful A Husain (2005). Algorithms for the recovery of Kohlrausch parameters from viscoelastic stress-strain data. ANZIAM 46:101-121.

9. Antoniadis A, Fan J (2001). Regularization of Wavelet Approximations. J Amer Stat Assoc 96:939-967. 
10. Banazsek D (2009). Optimization of the Measurement of the Linear Viscoelastic Properties of Complex Fluids. PhD thesis, Aberystwyth University, Wales.

11. Barnes HA, Hutton JF, Walters K (1989). An Introduction to Rheology. Elsevier, Amsterdam.

12. Bateman Manuscript Project. Tables of Integral Transforms. 1:30.

13. Baumgaertel M, Winter HH (1989). Determination of Discrete Relaxation and Retardation Time Spectra from Dynamic Mechanical Data. Rheol Acta 28:511-519.

14. Baumgaertel M, Winter HH (1992). Interrelation between continuous and discrete relaxation time spectra. J Non-Newton Fluid Mech 44:1536 .

15. Bernstein SN (1928). Sur les fonctions absolument monotones. Acta Mathematica 52: 166.

16. Bland DR (1960). The Theory of Linear Viscoelasticity. International Series of Monographs in Pure and Applied Mathematics. Pergamon Press.

17. Boltzmann L (1874). Sitzb Kgl Akad Wiess Wien 2 (Abt 70):725

18. Bowman AW, Azzalini A (1997). Applied Smoothing Techniques for Data Analysis: The Kernel Approach with S-Plus Illustrations. Oxford Statistical Science Series. Oxford Science Publications.

19. Calderón AP (1964). Intermediate spaces and interpolation, the complex method. Stud. Math., 24:113-190. 
20. Carrot C, Verney V (1996). Determination of a discrete relaxation spectrum from dynamic experimental data using the Pade-Laplace method. European Polymer Journal 32:69-77.

21. Chen SS, Donoho DL, Saunders MA (2001). Atomic decomposition by basis pursuit. SIAM Rev., 43:129-159.

22. Cho KS, Kim JW, Yeo H (2008). Determination of Discrete Relaxation Time Spectrum by Use of Continuous Wavelet Transform. AIP Conf Proc. 1027:1465-1467.

23. Chow MK, Zukoski CF (1995). Gap size and shear history dependencies in shear thickening of a suspension ordered at rest. J Rheol 39:15-32.

24. Chow MK, Zukoski CF (1995). Nonequilibrium behavior of dense suspensions of uniform particles - volume fraction and size dependence of rheology and microstructure. J Rheol 39:33-59.

25. Chui CK (1992). An introduction to wavelets. Academic Press, San Diego, USA.

26. Daubechies I (1992). Ten lectures on wavelets. Philadelphia, Pa: Society for Industrial and Applied Mathematics.

27. Davies AR , Anderssen RS (1986). Optimization in the regularization of ill-posed problems. J. Austral. Math. Soc. Ser. B 28:114-133.

28. Davies AR, Anderssen RS (1997). Sampling Localization in Determining the Relaxation Spectrum. J. Non-Newtonian Fluid Mech. 73:163179 .

29. Dealy JM, Larson RG (2006). Structure and Rheology of Molten Polymers. Hanser Gardner Publications, Cincinnati, Ohio. 
30. Elster C, Honerkamp J (1991). Modified maximum entropy method and its application to creep data. Macromolecules 24:310-314.

31. Elster C, Honerkamp J (1992). The role of the error model in the determination of the relaxation-time spectrum. J Rheol 36:911-927.

32. Engl HW, Hanke M, Neubauer A (1996). Regularization of Inverse Problems. Kluwer Academic Publishers, Dordrecht, Netherlands.

33. Ferry JD (1980). Viscoelastic properties of polymers. Wiley, New York.

34. Friedrich C (1991). A Delta-Function Method for the $n^{\text {th }}$ Approximation of Relaxation or Retardation Time Spectrum from Dynamic Data. Rheol Acta 30:7-13.

35. Fuoss R, Kirkwood J (1941). Anomalous dispersion and dielectric loss in polymers. J Chem Phys. 9:329-340.

36. Fuoss R, Kirkwood J (1941). Electrical Properties of Solids. VIII. Dipole Moments in Polyvinyl Chloride-Diphenyl Systems. J Amer Chem Soc. 63:385-394.

37. Gray RM, Olshen RA (1997). Vector quantization and density estimation. IEEE Comput. Soc, 172-193.

38. Groetsch CW (1993). Inverse Problems in the Mathematical Sciences. Vieweg Verlag, Wiesbaden.

39. Gröss B (1968). Mathematical structure of the theories of viscoelasticity. Hermann, Paris.

40. Hadamard J (1902). Sur les problmes aux drives partielles et leur signification physique. Princeton Uni. Bull., 13:49-52. 
41. Hansen S (2007). Estimation of the relaxation spectrum from $d y$ namic experiments using Bayesian analysis and a new regularization constraint. Rheol Acta 47:169-178.

42. Henrici P (1986). Applied and Computational Complex Analysis. John Wiley \& Sons, New York. Volume III.

43. Honerkamp J, Weese J (1989). Determination of the relaxation spectrum by a regularization method. Macromolecules 22:4372-4377.

44. Honerkamp J, Weese J (1993). A nonlinear regularization method for the calculation of relaxation spectra. Rheol. Acta 32:65-73.

45. Hussein WN (1997). Some inverse problems in rheology. PhD thesis, Aberystwyth University.

46. Kanwal RP (1983). Generalized functions: Theory and technique. Academic Press, New York.

47. Lighthill MJ (1958). An introduction to Fourier analysis and generalized functions. Cambridge University Press.

48. Loy RJ, Davies AR, Anderssen RS (2009). A revised duality proof of sampling localization in relaxation spectrum recovery. Bull. Aust. Math. Soc. 79: 79-83.

49. Macdonald JR (2000). On relaxation-spectrum estimation for decades of data: accuracy and sampling-localization considerations. Inverse Problems 16:1561-1583.

50. Malkin A.Ya. (2002). The sense of a relaxation spectrum and methods for its calculation. Vysokomol. Soedin. (Polymers in Russian) 44:1698-2005. 
51. Malkin A Ya (2006). The use of a continuous relaxation spectrum for describing the viscoelastic properties of polymers. Polymer Science Series A 48:39-45.

52. Malkin A Ya, Isayev AI (2006). Rheology, Concepts, Methods and Applications. ChemTec Publishing, Toronto.

53. Mallat S (2009). A Wavelet Tour of Signal Processing - The Sparse Way. Academic Press, San Diego, USA.

54. Maxwell JC (1867). Phil. Trans. Roy. Soc. Lond. 157:49-88.

55. Mead DW (1994). Numerical interconversion of linear viscoelastic material functions. J Rheol 38:1769-1795.

56. Mead DW (1994). Determination of molecular weight distributions of linear flexible polymers from linear viscoelastic material functions. J Rheol 38:1797-1827.

57. Miller A (1995). Subset selection in Regression. Monographs on Statistics and Applied Probability, Second Edition, Chapman \& Hall.

58. Morgan D (2003). Inverse Problems in Engineering Rheology. $\mathrm{PhD}$ thesis, Aberystwyth University.

59. Morosov VA (1984). Methods for solving incorrectly posed problems Spring-Verlag, New York.

60. Moulin P (2001). Regularization of wavelet approximations: Discussion. J Americ Stat Assoc 96:959-960.

61. Mustapha SMFDS, Phillips TN (2000). A dynamic nonlinear regression method for the determination of the discrete relaxation spectrum. Journal Of Physics D-Applied Physics 33:1219-1229. 
62. O'Brien VT, Mackay ME (2002). Shear and elongation flow properties of kaolin suspensions. J Rheol 46:557-572.

63. Parzen E (1962). On estimation of a probability density function and mode. Annals of Mathematical Statistics, 33:10651076.

64. Priestley HA (1985). Introduction to Complex Analysis. Clarendon Press, New York.

65. Prünte L (2008). Wavelet-dictionaries and continuous dictionaries. $\mathrm{PhD}$ thesis, Bremen University.

66. Saut JC, Joseph DD (1983). Fading memory. Arch. Rational. Mech. Anal. 81:53-95.

67. Schwarzl F, Staverman AJ (1953). Higher approximation methods for the relaxation spectrum from static and dynamic measurements of viscoelastic materials. Appl. Sci. Research, A4:127-141.

68. Shen X, Walter GG (2002). Meyer wavelet regularization. Numer Funct Anal and Optimiz 23:195-215.

69. Silverman BW (1986). Density Estimation for Statistics and Data Analysis. Monographs on Statistics and Applied Probability 26. Chapman and Hall.

70. Stadler FJ, Bailly C (2009). A new method for the calculation of continuous relaxation spectra from dynamic-mechanical data. Rheol Acta 48:33-49.

71. Tanner RI, Walters K (1998). Rheology: An Historical Perspective. Elsevier, Amsterdam. 
72. Thimm W, Friedrich C, Marth M, Honerkamp J (1999). An analytical relation between relaxation time spectrum and molecular weight distribution. J Rheol 43:1663-1672.

73. Tropp JA, Gilbert AC (2007). Signal recovery from random measurements via Orthogonal Matching Pursuit. IEEE, Transactions on Information Theory. 53:4655-4666.

74. Tschoegl NW (1989). The Phenomenological Theory of Linear Viscoelastic Behavior. Berlin, Heidelberg, New York, Springer-Verlag.

75. Van Ruymbeke E, Keunings R, Bailly C (2002). Determination of the molecular weight distribution of entangled linear polymers from linear viscoelasticity data. J Non-Newton Fluid Mech 105:153-175.

76. Walter GG, Shen X (1999). Deconvolution using Meyer wavelets. J Int Eqns 11:515-533.

77. Walter GG, Shen X (2000). Wavelets and other orthogonal systems. 2nd Ed., CRC Press: Boca Raton, FL.

78. Walters K (1975). Rheometry. Chapman and Hall, London.

79. Wineman AS, Rajagopal KR (2000). Mechanical response of polymers: an introduction. Cambridge University Press.

80. Wing GM, Zahrt JD (1991). A Primer on Integral Equations of the First Kind. City Press, Baltimore, Maryland.

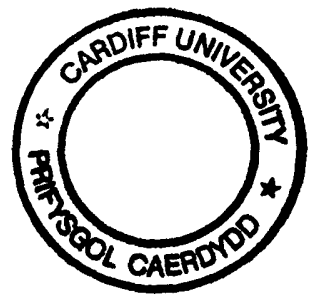

